

\title{
PNNL-15166
}

\section{EMSL Outlook Review 2005}

\author{
Dr. Allison Campbell
}

May 2005

Prepared for

the U.S. Department of Energy

under Contract DE-AC06-76RL01830

Pacific Northwest National Laboratory

Richland, Washington 99352 


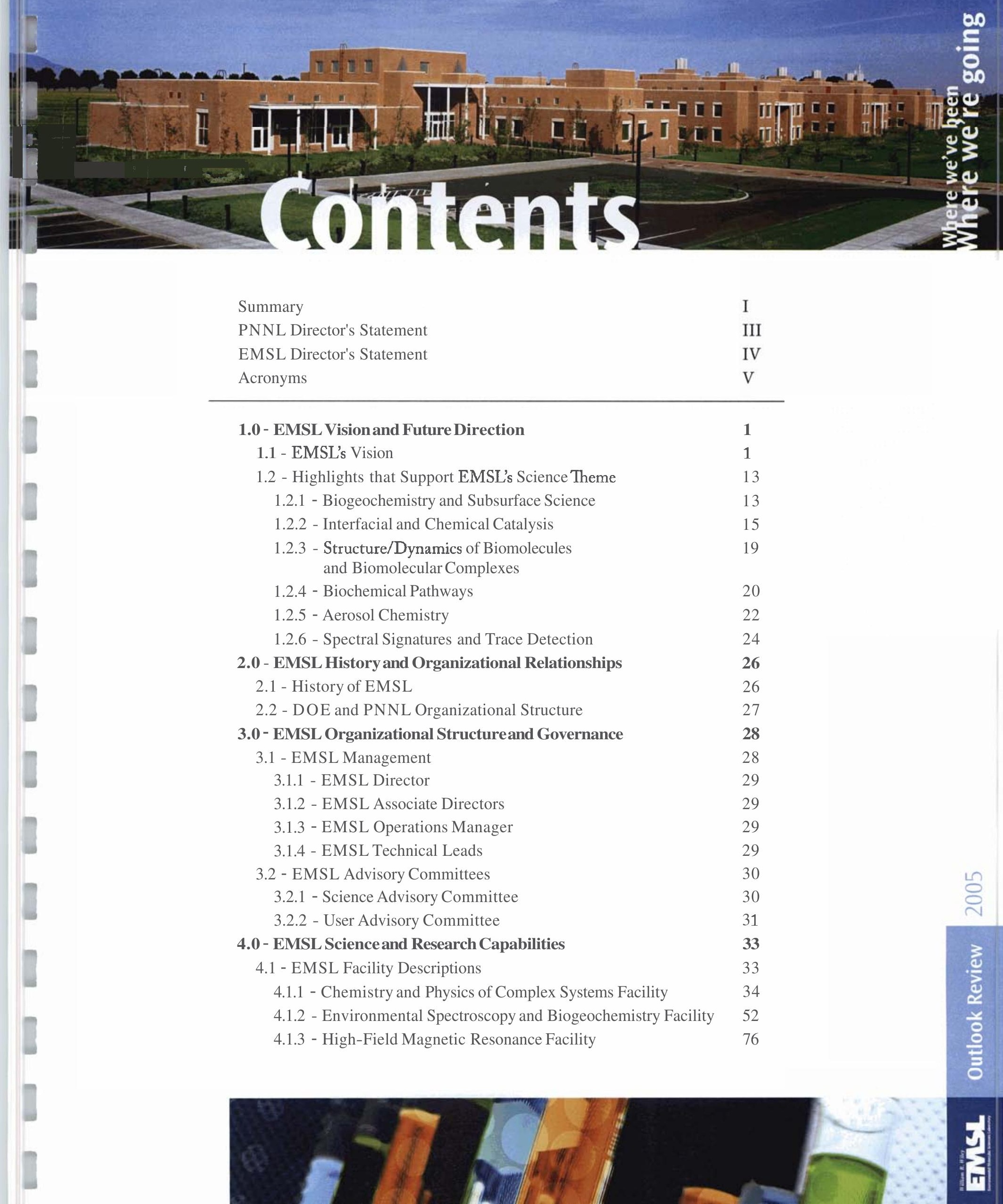




\section{Summary}

The William R. Wiley Environmental Molecular Sciences Laboratory (EMSL) is a national user facility that contains state-of-the-art instrumentation and expert resources available for use by researchers from academia, industry, and the national laboratory system. The facility is supported by the U.S. Department of Energy's (DOE) Biological and Environmental Research Program, but the research conducted within the facility benefits many funding agencies, including other branches of DOE, the National Institutes of Health, the National Science Foundation, and the Department of Defense.

EMSL's mission is to serve as a national scientific user facility that provides a collaborative environment for discovery and technological innovation in pursuit of the nation's most difficult and critical scientific challenges in the molecular and environmental sciences by:

- providing an integrated problem-solvingenvironment of scientific expertise, advanced instrumentation, and computational resources to scientists engaged in physical, chemical, and biological research

- engaging interdisciplinary and multi-institutional scientific teams to bring an integrated set of resources to bear on complex scientific problems

- educating students and scientists to meet the demanding multidisciplinary research challenges of the future.

EMSL requires the continued funding and support of its stakeholders and clients to continue to grow its mission, build its reputation as a sought-after national user facility with cutting-edge capabilities, and attract high-profile users who will work to solve the most critical scientific challenges that affect $\mathrm{DOE}$ and the nation. In this vein, this document has been compiled to provide these stakeholders and clients with a review document that provides an abundance of information on EMSL's history, current research activities, and proposed future direction. This document contains the following sections:

- Section 1 provides an overview of EMSL's vision and future direction, including research highlights that support EMSL's signature scientific themes.

- Section 2 provides an overview of the history of EMSL and its relationship to DOE and the Pacific Northwest National Laboratory — on whose campus EMSL resides.

- Section 3 introduces EMSL's management structure and its Science Advisory and User Advisory committees.

- Section 4 discusses the capabilities of EMSL's six research facilities and three support organizations, and provides highlights of the cutting-edge research performed during the last seven years at each facility.

- Section 5 provides an overview of EMSL's user outreach plan and the tools that drive user administration. 
- Section 6 provides an overview of EMSL user demographics and statistics of the types of publications and patents that result from user collaborations.

- Section 7 provides details on EMSL's operations budget.

In addition, a $\mathrm{CD}$ is provided that contains appendices detailing the roles, responsibilities, accountabilities, and authorities of EMSL management; descriptions of EMSL's research facilities and support organizations; the major equipment housed in EMSL; resumes and bios of key EMSL staff; testimonials provided by past and current users of EMSL; a list of users and associated projects; a list of publications generated by EMSL research; and a list of patents received as a result of EMSL research. 


\section{PNNL Director's Statement}

Welcome to the William R. Wiley Environmental Molecular Sciences Laboratory and Pacific Northwest National Laboratory. Your participation in the EMSL review is greatly appreciated, and I have no doubt that your feedback and ideas will help us to make this facility even more productive and dynamic in the years ahead.

EMSL is a remarkable research facility. But beyond its many capabilities and the exciting scientific discoveries that have emanated from its laboratories, EMSL has had a profound - perhaps revolutionary - impact on the whole of Pacific Northwest National Laboratory. In a relatively short time, EMSL has helped transform PNNL from a laboratory conducting highly regarded project engineering work to an outstanding science organization. We see evidence of this in our publications and external recognition. For two years running, our staff members have produced approximately 700 publications annually, with a substantial number of those papers appearing in pre-eminent journals. We also have received validation of our science through a growing number of prestigious awards and professional society appointments and recognition.

'This trend will continue. I envision EMSL and its resources serving as the core around which PNNL will perfect our new and innovative approach to researchsystems science. Systems science will integrate our strengths in physical, chemical, and biological sciences at the molecular level and across broad space and time scales, utilizing advances in computing, modeling, and engineering. I am confident that this methodology will shorten the path from discovery to solution and address needs in the fields of energy, the environment, and national and homeland security.

We have taken steps to position EMSL for this exciting future. I changed our organizational structure so that the director of EMSL now reports to my office. I believe this will strengthen the connection between PNNL's five research directorates and EMSL, while also cultivating collaborative interactions. Additionally, we are in the process of launching multi-institution scientific Grand Challenges in membrane biology and biogeochemistry at EMSL. These special research projects not only respond to the vision of our customers at the Department of Energy's Office of Science, but I believe they are going to spark some exciting breakthroughs. The Grand Challenges will certainly help PNNL and EMSL foster a climate of innovation that will benefit all future work.

Again, welcome and thank you for your efforts. Through your contributions of time, energy, and insight, you are helping to ensure that EMSL fully lives up to its great promise in serving the Department of Energy, the nation, and the world.

\section{Dr. Len Peters}

Director

Pacific Northwest National Laboratory

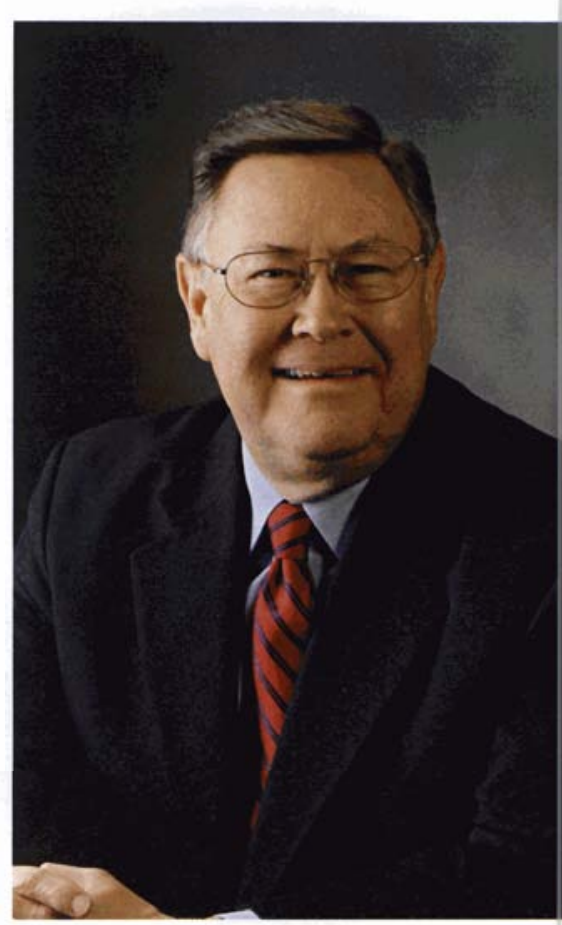

PNNL Director Dr. Len Peters 


\section{EMSL Director's Statement}

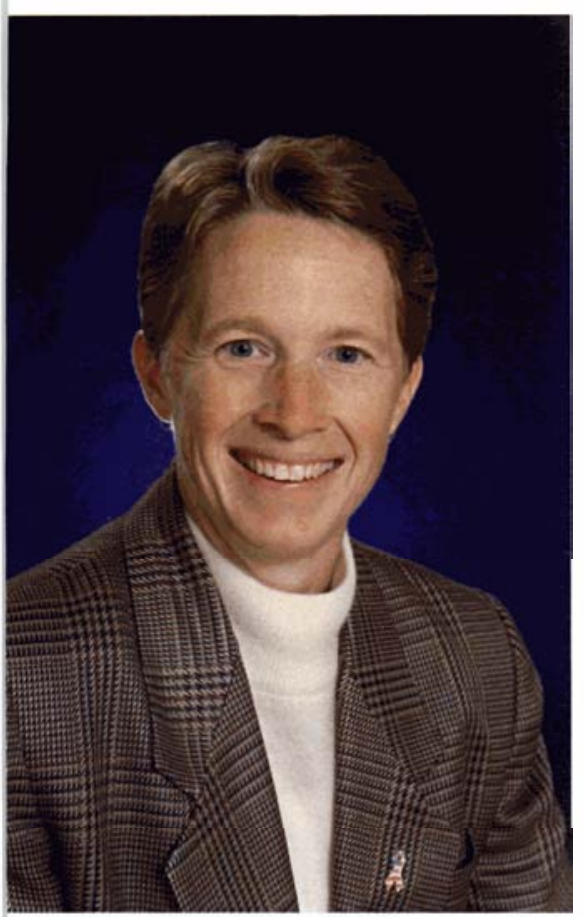

EMSL Director Dr. Allison Campbell
Since opening the doors of the William R. Wiley Environmental Molecular Sciences Laboratory in 1997, we have made extraordinary progress toward achieving our vision as the U.S. Department of Energy's premier national scientific user facility. In the past seven years, our scientific staff and users have been recognized nationally and internationally for their significant contributions to addressing challenging scientific problems. We have forged new scientific frontiers by organizing a vibrant and diverse user community and building new technical capabilities that support the molecular science basis for new knowledge in post-genomic biological research, catalysis, environmental materials, chemistry, and biology. Additionally, we continue our commitment to the education of students and scientists through robust undergraduate, graduate, anu postdoctoral research programs.

Our success is attributed to our commitment to three "signature" characteristics of EMSL as a national user facility:

1. The ability to integrate theory, modeling, and simulation with world-class experimental capabilities.

2. Innovative research and development teams that bring technology and concepts from many disciplines together to create integrated research tools and methodologies for new scientific challenges.

3. A collaborative mode of operation that promotes strategic partnerships to unite the strengths of EMSL with those of our users' home institutions, effectively leveraging these national investments.

Looking forward, we will focus on the nation's most important and challenging scientific problems via EMSL's Scientific Grand Challenges and Collaborative Access Teams, which capitalize upon EMSL's unique problem-solving environment, assets, and capabilities. We will push EMSL's strategic capabilities to new frontiers and create a transparent and supportive user environment to meet the challenges of multidisciplinary research. In this century, science and technology will be unified as never before, requiring a systems approach to problem solving and application. Our combination of world-class minds, methods, and capabilities uniquely positions EMSL to deliver answers to the questions that matter most to the science community and the nation.

\section{Dr. AllisonA. Campbell \\ Director}

William R. Wiley Environmental Molecular Sciences Laboratory 


\section{Acronyms}

\begin{tabular}{|c|c|}
\hline $\mathrm{AlON}$ & aluminum oxynitride \\
\hline AMP & adenosine monophosphate \\
\hline AMT & accurate mass and time \\
\hline CARS & coherent anti-Stoke Raman scattering \\
\hline CAT & Collaborative Access Team \\
\hline CCSEM/EDX & $\begin{array}{l}\text { controlled scanning electron microscopy with energy } \\
\text { dispersed analysis of x-rays }\end{array}$ \\
\hline CPMAS & cross-polarization, magic-angle spinning \\
\hline $\mathrm{CrON}$ & chromium oxynitride \\
\hline DAGK & diacylglycerol kinase \\
\hline DFT & density functional theory \\
\hline DNAPL & dense nonaqueous phase liquid \\
\hline DOE & U.S. Department of Energy \\
\hline Ecce & Extensible Computational Chemistry Environment \\
\hline EMSL & $\begin{array}{l}\text { William R. Wiley Environmental Molecular } \\
\text { Sciences Laboratory }\end{array}$ \\
\hline ELDOR & electron double resonance \\
\hline ENDOR & electron nuclear double resonance \\
\hline EPR & electron paramagnetic resonance \\
\hline FPGA & field-programmablegate array \\
\hline FSAM & fluorinated self assembled monolayer \\
\hline FTICR & Fourier-transform ion cyclotron resonance \\
\hline GA Tools & Global Array Tools \\
\hline GFP & green fluorescence protein \\
\hline GGA & generalized-gradient approximation \\
\hline HRTEM & high-resolution transmission electron microscopy \\
\hline MBE & molecular beam epitaxy \\
\hline MOCVD & metalorganic oxide chemical vapor deposition \\
\hline MS/MS & tandem mass spectrometry \\
\hline
\end{tabular}




$\begin{array}{ll}\text { NESG } & \text { Northeast Structural Genomics Consortium } \\ \text { NMR } & \text { nuclear magnetic resonance } \\ \text { NUC } & \text { natural uranyl-bearing calcite } \\ \text { NWChem } & \text { Northwest Chemical Computation Software } \\ \text { PETSc } & \text { Portable Extensible Toolkit for Scientific computation } \\ \text { PNNL } & \text { Pacific Northwest National Laboratory } \\ \text { PMAP } & \text { plasma membrane-associated protein } \\ \text { QCPMG } & \text { Quadrupole Carr-Purcell Meiboom Gill } \\ \text { RTK } & \text { receptor tyrosine kinase } \\ \text { SAM } & \text { self-assembled monolayer } \\ \text { SAMMS } & \text { self-assembled monolayers on mesoporous silica } \\ \text { SEM } & \text { scanning electron microscopy } \\ \text { SIC } & \text { self-interaction correction } \\ \text { SIMS } & \text { secondary ion mass spectrometry } \\ \text { SSNMR } & \text { solid-state nuclear magnetic resonance } \\ \text { STOMP } & \text { Subsurface Transport Over Multiple Phases (computer model) } \\ \text { TEM } & \text { transmission electron microscopy } \\ \text { TMD } & \text { transmembrane domain } \\ \text { TPD } & \text { temperature programmed desorption } \\ \text { TRAPDOR } & \text { transfer of population by double resonance } \\ \text { TRLFISM } & \text { time-resolved laser-induced fluorescence imaging } \\ \text { TRLFS } & \text { spectromicroscopy } \\ \text { TOF } & \text { time-resolved laser-induced fluorescence spectroscopy } \\ \text { XPS } & \text { timelectron spectroscopy } \\ \text { THEction }\end{array}$




\section{EMSL's Vision and Future Direction}

\section{1}

\section{EMSL's Vision}

The William R. Wiley Environmentaland Molecular Sciences Laboratory (EMSL) is the sought-afterleader in developing solutions to complex challenges of national importance. Through multidisciplinary collaborations with an engaged, proactive user community; expert scientists and staff; and state-of-the-art signature capabilities, we will deliver accelerated, leadership-class accomplishments.

EMSL's mission is to serve as a national scientific user facility that provides a collaborative environment for discovery and technological innovation in pursuit of the nation's most difficult and critical scientific challenges in the molecular and environmental sciences by:

- providing an integrated problem-solvingenvironment of scientific expertise, advanced instrumentation, and computational resources to scientists engaged in physical, chemical, and biological research

- engaging interdisciplinaryand multi-institutional scientificteams to bring an integrated set of resources to bear on complex scientific problems

- educating students and scientists to meet the demanding multidisciplinary research challenges of the future.

EMSL's strength and uniqueness as a national user facility is in providing a systems science approach and a problem-solving environment to bear on complex scientific problems. This approach is accomplished through our three signature characteristics:

1. 'The integration of theory, modeling, and simulation with world-class experimental capabilities.

2. Innovative research and development teams that bring technology and concepts from many disciplines together to create integrated research tools and methodologies for new scientific challenges.

3. A collaborative mode of operation that promotes strategic partnerships to unite the strengths of EMSL with those of our users' home institutions, effectively leveraging these national investments.

EMSL research is focused around scientific themes in the following areas. More detail on these scientific themes, and an example highlight of work performed at EMSL in each theme, are provided below:

- Biogeochemistry and Subsurface Science

- Interfacial Chemistry and Catalysis

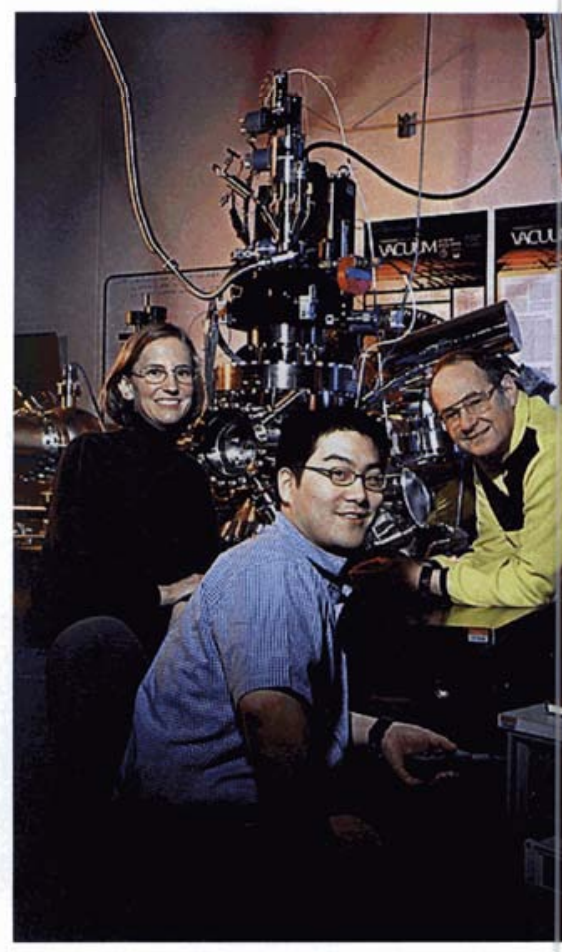


- Structure/Dynamics of Biomolecules and Biomolecular Complexes

- Biochemical Pathways

- Aerosol Chemistry

- Spectral Signatures and Trace Detection.

\section{Paradigm Shift}

'The early years in EMSL's evolution involved building its reputation as a national scientific user facility. 'This meant developing and establishing leadership-class instrumentation and capabilities, using those capabilities to build a scientific reputation, and establishing a robust user program. Today, EMSL has succeeded - it is widely recognized for its cutting-edge capabilities, world-class science, and thriving user program.

Critical scientific challenges that affect our nation have arisen during the $21 \mathrm{st}$ Century; these challenges require a more concerted systems science approach to problem solving and scientific discovery. In response, EMSL is focusing on these challenges through a set of Scientific Grand Challenges and Collaborative Access Teams (CATs) (discussed in detail in Sections 4.3 and 4.4), which will push EMSL's strategic capabilities to new frontiers and create a transparent and supportive user environment needed to meet the challenges of multidisciplinary research. This requires a paradigm shift from predominantly single investigator user projects to research teams highly focused on large scientific problems. To enhance EMSL's impact and create an integrated problem-solving environment, efforts must be focused around the scientific strengths of EMSL staff and resident users.

To achieve its vision, EMSL has adopted a set of strategic goals to keep its researchers and users focused and refreshed in order to successfully address highimpact scientific challenges:

1. Establish distinctive science signatures

2. Focus on the nation's most challenging scientific problems within EMSL's scope

3. Maintain EMSL's strategic capabilities at the scientific forefront

4. Enhance EMSL's facilities to support world-class science.

\section{Goal 1: Establish Distinctive Science Signatures}

The six research areas outlined below highlight potential scientific themes that represent EMSL's strengths and areas where the user facility has achieved considerable recognition. Additionally, these areas are the mechanisms for engaging EMSL's senior resident users in helping to drive science forward. Each theme will be stewarded by senior scientists who will help establish the scientific focus and the capabilities that will be needed in support of the scientific theme, and who will help to build a user community of prestigious scientists.

Each subsection below describes the scientific drivers and EMSL's niche in each scientific area. In addition, science highlights are provided in section 1.2 that illustrate EMSL's capabilities in the scientific theme area. 
These themes are currently under development and may change somewhat in the coming year as EMSL fully develops its strategic plan. All proposed themes will be vetted through the U.S. Department of Energy (DOE) - the sponsor of EMSL_as well as EMSL's Science Advisory Committee.

\section{Scientific Theme 1: Biogeochemistry and Subsurface Science}

Chemical processes in soil and subsurface systems regulate the extent and rate of contaminant migration and transformation, fluxes of carbon and other bioessential elements, chemical speciation as a control on bioassimilation and uptake, and overall environmental reactivity. For this reason, the study of soil and subsurface cbemicalprocesses is crucial to various DOE mission areas, including environmental restoration, actinide chemistry, carbon sequestration, global atmospheric dynamics, and tk development of "green" technologies.

EMSL has been a national leader in various aspects of soil and subsurface science during the past decade, with research that has focused on 1) the biogeochemistry of mineral (oxide/clay)-microbe systems; 2) reaction processes at the mineralwater interface; and 3) thermodynamic and molecular models of actinides, mineral surfaces, bacterial membranes, and high-ionic strength systems.

Unique capabilities in computation and spectroscopy place EMSL in a strong position to address various fundamental science issues of future importance to DOE. EMSL's competitive nick is in elucidatingmolecular-levelreactions at mineraland microbe interfaces through molecular modeling and spectroscopy, and $\boldsymbol{t} \boldsymbol{k} \boldsymbol{n}$ validating theircontribution to reactivity at tk macroscopic scale and in natural materials through experimentation and computation.

\section{Scientific Theme 2: Interfacial Chemistry and Catalysis}

As in all catalyst research, a fundamental understanding of the chemical reaction mechanisms that occur on the catalyst surface, including an identification of the catalytic sites directly involved in the rate-limiting elementary processes, is critically important to rationally develop improved catalysts and catalytic processes. EMSL's capabilitiesin surface science (synthesis, characterization, and modeling) provide a competitive niche to address the fundamentalaspects of the ckmical reaction mechanism @ that occur on the catalystsurface, particularlywith respect to oxide-based catalystmaterials. These capabilities are founded upon a world-class program in condensed-phase interfacial chemistry and are coupled with facility and expert resources unique to DOE's national laboratory system that allow basic research results to be applied to programs aimed at catalyst material and process development.

State-of-the-art computational methods of chemistry, solid-state physics, and materials science are used to interpret experimental results and design new materials with favorable catalytic properties, with the goal to provide insight at the atomic level of the interaction of solids with adsorbed molecules and the behavior of catalytic sites. Furthermore, EMSL's nuclear magnetic resonance (NMR), electron spin resonance, and spectroscopic capabilities

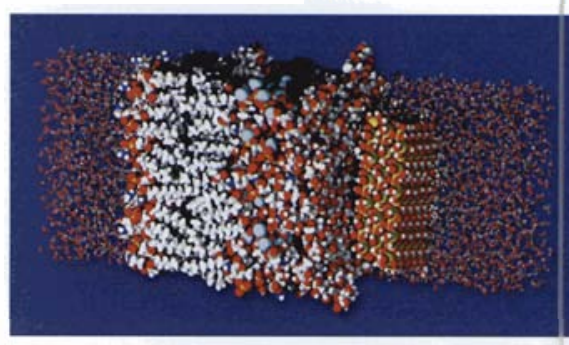

Electrostatic potential isocontours of the rough LPS membrane of pseudomonas aeruginosa

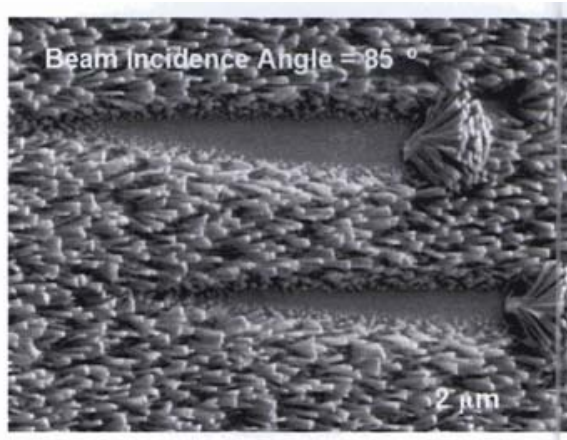

Nanoporous Mg0 film synthesized using Reactive Ballistic Deposition 


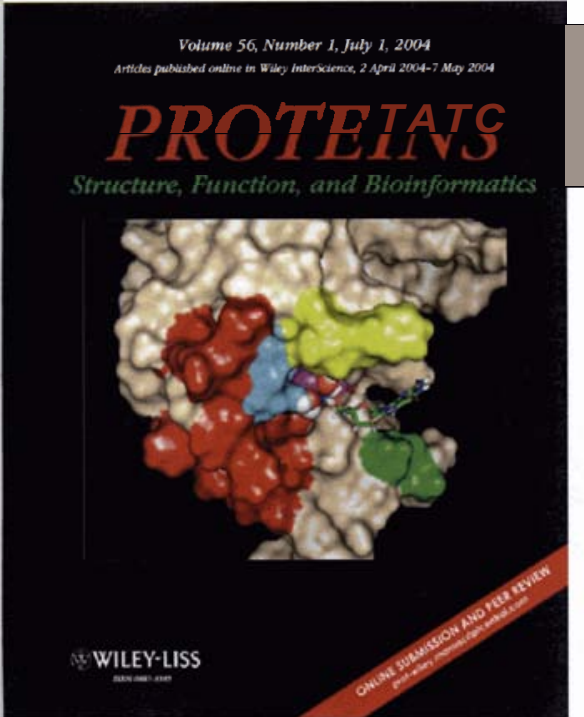

"Superbug" protein. The resistance of D. radioduransto radiation and to other DNAdamaging agents is hypothesized to involve its unusually high number of "surveillance and housecleaning" enzymes called Nudix hydroylases. The NMR structure of the Nudix hydrolase DR0079 was featured on a recent cover of Proteins. Shown is a proposed model for the binding of a generic substrate (nucleoside and diphosphate, atomic colors) and hydrated, divalent metal cation ion (pink) to the solution structure of DR079. have great potential for directly providing a fundamental characterization of the relationship between structure and reactivity of heterogeneous catalysts. Understanding how chemicals interact at the surfaces of catalyst materials is the key to converting energy into chemicals and vice versa, and can be applied to developing industrial and environmental solutions. Further, DOE's environmental and energy mission can be impacted through development of catalyst materials andprocesses for efficient use offossilfuels, automobile catalytic converter devices, green chemistry use of $\mathrm{CO}_{2}$, andproduction of value-added chemicals and fuels from biobasedfeedstocks.

\section{Scientific Theme 3: Structure/Dynamics of Biomolecules and Biomolecular Complexes}

A molecular-scale understanding is required for a science-to-solutions approach to biological systems. EMSL's unique capabilitiesin imaging technology, computationalmodeling,and bioinformatics enable an enhanced molecular-level understanding of the underlyingphysical,chemical, and biologicalprocesses by allowing researchers to visualize the structures, processes, and interactions of these molecules in detail. These capabilities will contribute to an understanding of the links among structures, dynamics, and functions of complex biological molecules such asDNA and proteins, and the behavior of the cells containing these molecules. Experimental methods include high-field solution and solid-state NMR, pulsed electron paramagnetic resonance (EPR), optical and laser spectrometry, molecular and cellular imaging, and molecular theory. Development and application of novel imaging techniques enable the study of selected metabolic pathways (cellular signaling pathways) and the effects of perturbations in live cells in more detail than was possible before. Understanding structure-function relationshipsis important, as it wouldprovide a means to begin understanding molecularprocessesin environmental remediation, wasteprocessing, and human health.

\section{Scientific Theme 4: Biochemical Pathways}

One critical fundamental science issue - a better understanding of biochemical pathways - is required for gaining a molecular-level understanding of how cells work. This is critical if microbial systems are to be engineered to be more effective for bioremediation, carbon sequestration, or other environmental uses. It is also critical for the effective identification of biomarkers and signatures. Biomolecular signatures and biomarkers - such as a set of expressed proteins, metabolites, and/or lipids in an organism - when examined together, present a unique pattern of molecular change that identifies an exposure or response to a specific environmental exposure or environmental stress. EMSL's niche in this area is its high-throughput proteomics capability centered around high-jield mass spectrometry, high-jield NMR microscopycapabilities, and computational methods, which allow researchers to gain a deeper understanding of these biochemicalpathways. EMSL's High-Performance Mass Spectrometry Facility is a leader in the development and application of new tools for measuring and modeling protein abundances and/or modifications as a function of organism development, perturbation, and/or environmental stress. EMSL's High-Field 
Magnetic Resonance Facility provides unique tools for probing biomolecular structures and dynamics. Bioinformatic tools for large-volume mass spectrometry data manipulation and higher-order data visualization suitable for computational biology model testing and validation, as well as new tools for the analysis of high-throughput proteomic data to abstract out gene and protein networks and other biological networks, are being developed to cultivate realistic models of living cells. Technology development in concert with its applications has provided a range of new insights into the proteins actually expressed by organisms under various conditions. Applications include understanding anaerobic microbial metabolism, which is of directrelevance to the DOE missions in environmental stewardship (contaminantbioremediation, microbial impacts on global warming through production and sequestration of methane and carbon dioxide); clean and secure energy (methane and $\mathrm{H}_{2}$ from wastes as alternative energy sources); and basic science (cycling ofcarbon, nitrogen, metals, and radionuclides).

\section{Scientific Theme 5: Aerosol Chemistry}

\begin{abstract}
EMSL's capabilities in instrument development and surface chemistry are extremely valuable contributions to the field of atmospheric chemistry Specifically, EMSL is well positioned to focus on the chemical reactions of atmospherically important organic compounds on the surfaces of carbonaceous (specifically, combustion-related) atmospheric aerosols.
\end{abstract}

EMSL's strength is its ability to make fundamental measurements tbat advance the understanding of aerosol cbemistry combined with its capacity for coupling laboratory experiments and field measurements witb modeling and simulation. EMSL has pushed the state of the art in atmospheric chemistry measurements using techniques such as scanning electron microscopy (SEM), time-offlight-secondary ion mass spectrometry (TOF-SIMS), sampling, and proton reaction transfer mass spectrometry. Research efforts are aimed at obtaining measurements that provide an understanding of fundamental processes, apply existing instrumentation to characterize the critical quantities, build and/or develop new capabilities, deploy these capabilities to field campaigns, and provide the theoretical framework for understanding the measurements while feeding values obtained for key parameters back into models. Understanding aerosol su face information could be used to improve the treatment of beterogeneous chemistry in air quality and climate models and to understand the effects of atmospheric processing on the chemical composition of atmospheric aerosols.

\section{Scientific Theme 6: Spectral Signatures and Trace Detection}

'The collection of high-quality, quantitative data associated with the detection of nuclear, chemical, and biological weapons manufacturing, storage, delivery, and degradation in the environment is of vital importance to DOE and other government agencies. Additionally, understanding how these threats can affect ecosystem health and recovery from chemical, biological, radiation, and nuclear threats and acts is of paramount importance. EMSL's niche is in the development

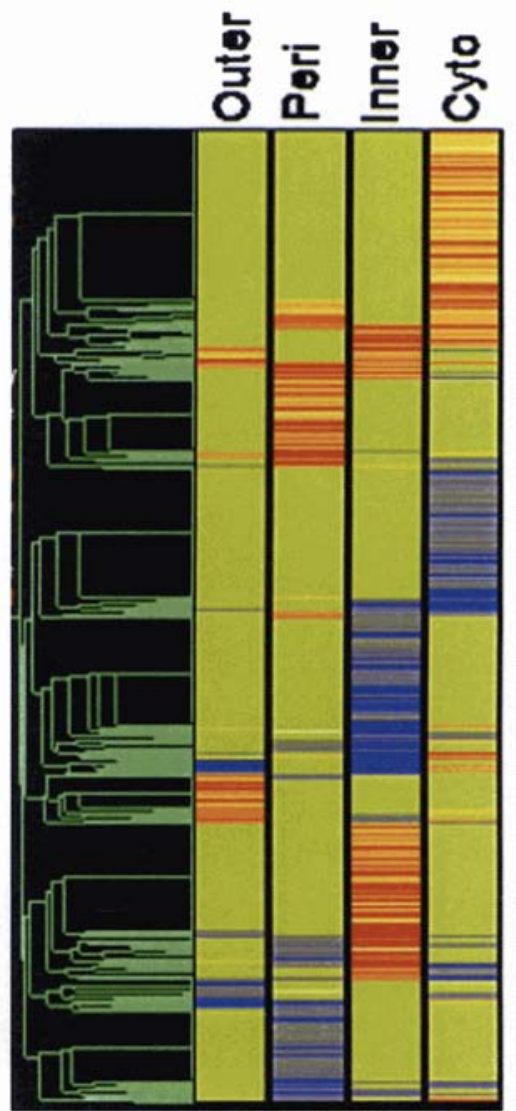

Clustering analysis of protein expression changes in Rhodobactersphaerodies grown under anaerobic and photosynthetic conditions. The cells were fractionated into the inner and outer membranes, the periplasm and the cytoplasm. Such analysis identifies groups of proteins that are important for understanding how this microbe performs its environmentally important roles of metal reduction and carbon sequestration. 


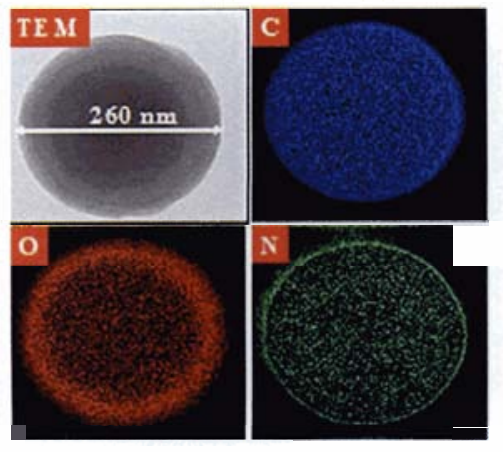

Electron energy loss spectroscopy shows elemental maps of tar ball including oxygenated rim. of world-class spectroscopy capabilities with unique instrumentation for signatures measurements combined with extensive expertise in spectraldata and its

interpretation. EMSL researchers use this capability to successfully demonstrate that trace amounts of heavy molecules in the vapor phase can be detected using high-resolution solid-state lasers. Today, EMSL researchers have received funding from DOE's National Nuclear Safety Administration to develop a spectral "library ${ }^{\mathrm{n}}$ that is distributed to 60 to 80 U.S. government entities who use the library as the definitive reference for chemical detection (http://nwir.pnl. gov) Ongoing use of such equipment and capabilities by EMSL users and their Pacific Northwest National Laboratory (PNNL) collaborators continues to drive technology to new levels of performance in search of answers to fundamental science questions. Additionally, EMSL scientists have demonstrated the ability to discriminate between bacterial growth media by analysis of metals signatures of bacterial spores. Identification and understanding of spectral signatures supports DOE's mission of enhancing the capability to detect weapons of mass destruction, including nuclear, chemical, and biological systems.
Vapor phase infrared spectral lihran

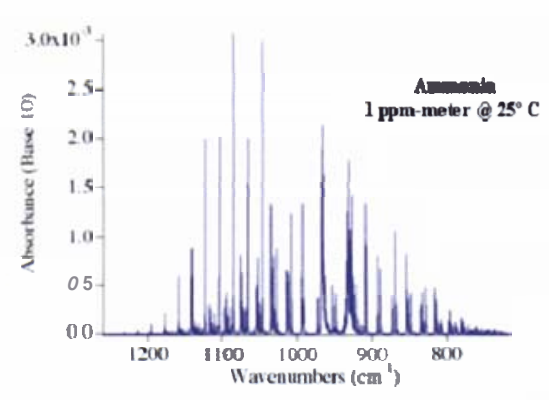

Database Summary Paper

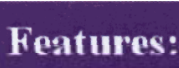

- Speriral coverage fiom 600 io $6500 \operatorname{cin}^{-106.16}$ ta 1.53 jum

- Composite spectra based an Reer's han Di of 5 or unere burtens

- Many spectra supplied at 5,25 and $50^{\circ} \mathrm{C}$

- All spectra inclode full doc unentation and rapor pressure versus temperature eurves

- One tine low price of \$200 for unlimibe d acress

Constantly growing list whth over 400 sompounde planned
EMSL-developed spectral library
Goal 2: Focus on the Nation's Most Challenging Scientific Problems within EMSL's Scope
EMSL's problem-solving approach provides an environment for large research activities that users could not accomplish elsewhere. A multifaceted approach to a particular scientific problem can be achieved by EMSL's users due to the ease and flexibility in integrating experimental and computational components of its core facilities. Additionally, staff quality and a willingness to collaborate with and assist users are key features that distinguish EMSL as a preferred location for users to pursue new or difficult scientific multi- or cross- disciplinary investigations.

To achieve this goal, EMSL is strengthening and focusing its user program through Scientific Grand Challenges and science-driven CATs.

\section{Scientific Grand Challenges}

EMSL provides an environment and cutting-edge capabilities that enable teams of users to focus on important scientific challenges that require a significant fraction of EMSL resources for considerable periods of time. To facilitate this environment, EMSL is implementing Scientific Grand Challenges coordinated, multi-investigator research focused on resolving a major scientific problem. 'Ihese Scientific Grand Challenges will:

- Focus on critical milestones in the advancementor use of science

- Support DOE mission areas 
- Be user driven

- Take full advantage of EMSL's unique resources and technical expertise

- Increase the scientific impact and robustness of EMSL as a user facility.

The scope of the scientific problems to be addressed will involve multidisciplinary teaming, and the breadth of a challenge will be of such magnitude that it cannot be addressed by any other existing single facility or by any single investigator. The Scientific Grand Challenges will require not only the capabilities of EMSL, but possibly other DOE user facilities and specialized instrumentation and capabilities at the participants' home institutions. They will also require significant management and coordination to meet their aggressive goals and are expected to attract and involve users who are among the best scientists in the world.

Currently, two Scientific Grand Challenges are

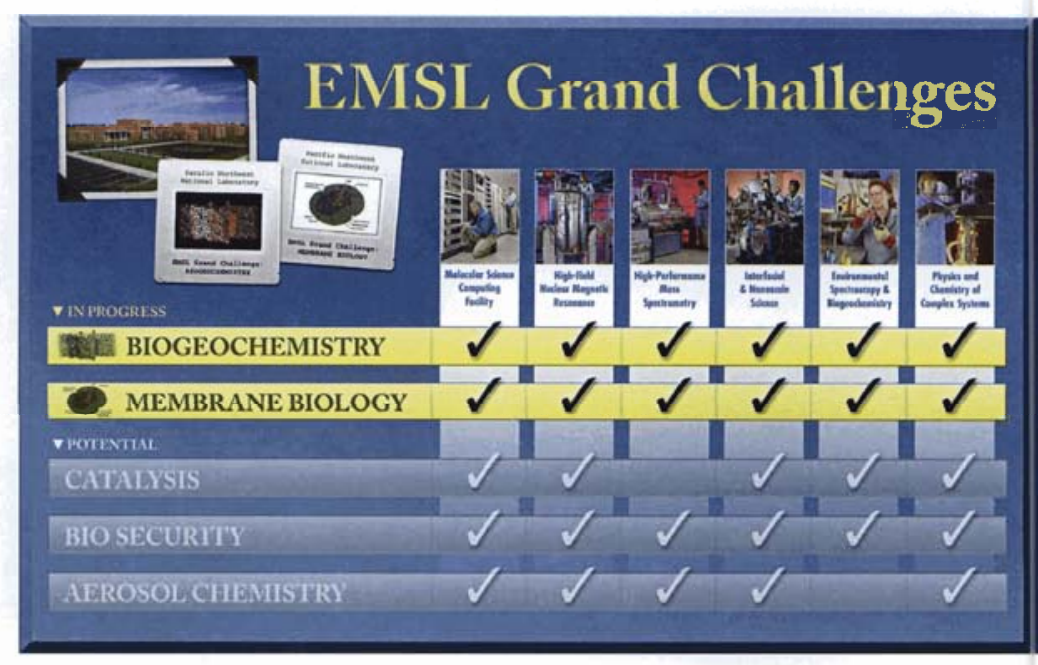
being conducted, one in biogeochemistry and the other in membrane biology. Detailed discussions about these Scientific Grand Challenges are provided in Section 4.3. Additionally, EMSL is working with DOE and its user community to define potential future Scientific Grand Challenge themes, as shown to the right.

\section{Science-Driven CATs}

In addition to EMSL's Scientific Grand Challenges, the scientific impact of the user program increased by focusing groups of users on important scientific problems that require a significant fraction of the resources in EMSL for considerable periods of time. This effort is facilitated through the formation of CATs, which are comprised of scientists who use EMSL facilities and capabilities to conduct funded programmatic research around focused science themes. CATs provide a mechanism to attract and increase the number of high-impact users in a focused research area and to build new capabilities for use by the CATs and the user community at large. Participation in a CAT is open to members of the scientific community who can contribute significantly to the scientific goals of the CAT. Many members of the user community may choose to gain access to EMSL through a CAT rather than by standard user proposals or other gateways.

EMSL is deploying four CATs:

- Structural Genomics: 'This CAT is carrying out structural genomics research focused on high volumes of protein structure data and is testing the feasibility of high-throughput, NMR-based structural genomics.

- Catalysis: 'This CAT provides an integrated experimental and theoretical approach to obtaining a molecular-level understanding of oxide-catalyzed chemical transformations. 
a Analytical Mass Spectrometry: The general objective of this CAT is to achieve a fundamental understanding of the reaction kinetics and dynamics of activating and dissociating complex molecular ions. 'These processes are the scientific foundation for tandem mass spectrometry (MS/MS), one of the most important analytical methods developed in the last century.

- Atmospheric Emissions: The purposes of this CAT are to create a unique research capability with an international user base and to understand the complex aerosol surface chemistries and its policy implications.

Details on each CAT are provided in Section 4.4.

\section{Goal 3: Maintain EMSL's Strategic Capabilities at the Scientific Forefront}

In EMSL's intense user environment, key instruments are highly used, so investments in upgrades for enhanced productivity, efficacy, or new capability are strongly leveraged against the national investment in the user community. Ongoing capital investments at EMSL for upgrading select systems under high use in EMSL distinctive science signatures, Scientific Grand Challenges and CAT research are needed to increase EMSL's productivity and scientific impact and help establish even greater user participation. The establishment of an annual inflation-adjusted capital budget of 4 percent of the replacement value for EMSL equipment $(\sim 100 \mathrm{M})$ is needed so that EMSL capabilities do not continue to fall behind their scientific usefulness. 'This level of ongoing funding is recommended by EMSL's Science Advisory and User Advisory committees and is consistent with the recommendations found in the Final Draft Report of the Secretary of Energy Advisory Board's (SEAB) Task Force on the Future of Science Programs at the Department of Energy, "Critical Choices: Science, Energy, and Security" (October 13,2003). This level of funding represents approximately 15 percent of the annual EMSL operations budget (less the allocation for the Molecular Science Computing Facility supercomputer lease).

During the past several years, EMSL management has made several strategic investment decisions relative to refreshment of the facility. By investing EMSL resources in a focused manner in areas of its distinctive science signatures and in areas of interest to D OE Office of Biological and Environmental Research programs, and by leveraging the investment of other agencies, EMSL has been able to make some progress toward refreshment.

\section{Advanced Planningfor Capability Development}

The driving force and focus for generating new capabilities is in the implementation of EMSL Scientific Grand Challenges, the deployment of CATs, and EMSL's scientific themes. When possible, EMSL capital or operations funding will be leveraged with programmatic resources. Beginning in Fiscal Year 2004, EMSL allocated annually $\$ 600 \mathrm{~K}$ of its operations budget to strategic capability development (in addition to the capital equipment budget). 


\section{Leveraging Investments with Other Agenciesand DOE Offices}

Establishing partnerships with other DOE offices and government agencies has proven to be an effective means of refreshing instrumentation. For example, in the past two years, the DOE Office of Science's Basic Energy Sciences Program has cost shared with EMSL to develop the following capabilities:

- An applied-fieldMossbauer spectrometer that supports resident users funded by the Basic Energy Sciences Program as well as a host of external users interested in the identification and quantification of iron (oxidation, coordination states) in geochemical, soil, and materials sciences. Additionally, this instrument represents a unique capability, as less than 5 percent of the few existing Mossbauer laboratories possess an applied-field spectrometer.

- A photo-electronemission microscope used for surface, interface, and catalysis studies. Leveraged funding ( $\$ 250 \mathrm{~K}$ capital from the Basic Energy Science's Catalysis Program and $\$ 150 \mathrm{~K}$ from EMSL's capital budget) was used to procure this instrument. 'This instrument provides an entirely new capability for imaging surfaces of metals, semiconductors, and thin films on the nanometer-length scale.

- A variable-temperature, ultrahigh vacuum, noncontact atomic force microscope/scanning tunneling microscope used for single-site surface chemistry/catalysis studies. Leveraged funding $(\$ 275 \mathrm{~K}$ capital from the Basic Energy Sciences Program's Chemical Sciences Capital and \$275K from EMSL's capital budget) was used to establish this capability, which allows researchers to develop a fundamental scientific understanding of sitespecific chemistry in surface science and catalysis studies.

In addition, EMSL has partnered with DOE's National Nuclear Safety Administration to enhance its high-resolution Fourier transform infrared spectrometry capability with the procurement of a system for analyzing molecules and clusters. The system, purchased using leveraged funding ( $\$ 150 \mathrm{~K}$ of EMSL capital budget and $\$ 150 \mathrm{~K}$ from the National Nuclear Safety Administration), provides updated capability and increased throughput and places EMSL's growing infrared spectroscopy programs at the international forefront.

EMSL has also leveraged its investments in its High-Field Magnetic Resonance Facility via the Structural Genomics CAT, which has provided a $600-\mathrm{MHz}$ NMR spectrometer with cold probe that will be available to EMSL users. The system was purchased with Battelle and National Institutes of Health funding. Additionally, EMSL will invest $\$ 300 \mathrm{~K}$ of capability development dollars to build a high-throughput system that will potentially increase throughput by three to four times.

Finally, EMSL's world-class global proteomics capability would not have been possible without very significantinvestments by other parts of the DOE Office of Biological and Environmental Research Program and other government agencies. An initial EMSL investment established the user facility as a leader in the rapidly developing field of proteomics. However, to stay at the forefront in this critical field required continuous developments in sample handling, mass spectrometry, and data analysis and management capabilities. This investment exceeded the 
funding the program was able to provide, so other funding sources were found. 'These new funds were then used to develop the Proteomics Research Information Storage and Management data management/analysis system and most of the sample-handling protocols, and to acquire six of EMSL's eight ion trap mass spectrometers (including all three of the newest linear ion trap systems) and two of the three Fourier transform ion cyclotron resonance (FTICR) mass spectrometers. In addition, these other funding sources partnered with EMSL to develop the newest FTICR mass spectrometer that currently constitutes EMSL's global proteomics capability.

\section{Adopt a Best Practice for Future Upgrades to the Molecular Science Computing Facility}

The Molecular Science Computing Facility specializes in developing and delivering science-driven computer architectures, software, and scientific expertise. Future upgrades will enable the faciity to further expand into new scientific areas, including systems biology, bioinformatics, climate modeling, and subsurface fate and transport modeling, while maintaining leadership in computational chemistry. High-performance computers have a typical life span of three to four years, while computer performance tends to double every 18 months (Moore's Law). Given a budget of $\$ 9 \mathrm{M}$ per year, it is possible to deliver a system that will enable competitive science during the next 10 years. From Fiscal Years 2000 to 2003, the Molecular Science Computing Facility demonstrated that a three- to four-year lease-to-own procurement and deployment cycle was a best practice for DOE scientific computing. EMSL has adopted this cycle by documenting the science drivers, partnering with vendors, and delivering systems every three to four years to keep the facility at the forefront of computational science. In addition, the science drivers also determine investments in theoretical and computational methods to be developed in the software to enable users to make use of the high-performance computer resources.

\section{Develop a Renewal, Refreshment, and Retirement Plan and Timeline}

Since EMSL's commissioning in 1997, the laboratory and its facilities have been vital to thousands of external users in pursuit of the highest-quality science. While some of the major instruments have undergone upgrades during EMSL's first six years of operation, many of the systems require further upgrades or nearterm replacement. While not all instruments at EMSL need to be maintained at the cutting edge, in order to maintain productivity and value to the scientific community, many of the instruments must be able to address today's scientific questions with the appropriate sensitivity, selectivity, and resolution.

Using its Scientific Grand Challenges, CATs, and distinctive science signatures as a focal point, EMSL is currently establishing a capital refreshment plan and timeline that will illustrate the anticipated useful scientific lifetime for a given capability, estimate upgrade and/or replacement costs, and highlight the new and emerging science enhancements that will result from refreshment. Additionally, EMSL will identify capabilities are no longer needed to meet anticipated user demand and will retire that instrumentation. 


\section{Goal 4: Enhance EMSL's Facilities to Support World- Class Science}

\section{Increased Raised Floor for Expanded Computational Needs}

EMSL plans to add approximately 4000 square feet of raised floor space to its existing 5000 square feet. Current plans call for increasing archive storage capabilities to approximately two petabytes by Fiscal Year 2009. This increase is necessary due to the large amount of data generated by EMSL's high-throughput proteomics research, which produces 10 gigabytes of data in a single experiment, and the Molecular Science Computing Facility, which can generate terabytes of data in single simulations. Storage needs will increase as EMSL combines image data with computation and as it enters into high-throughput image data analysis and management in support of its Scientific Grand Challenges.

Such increased storage needs will require as much as 60 additional rack locations, or approximatelyone-third of the current 5000 square feet of floor space. The planned expansion will accommodate this need and allow equipment currently occupying other EMSL laboratory space to be consolidated in the central raised floor space. A portion of the expanded floor space will also be used to build the first phase of the next-generation computing hardware to facilitate an orderly user migration without the need to dismantle part of the existing computing capability.

\section{Machine Shop Enhancements}

Machinists located in EMSL's machine shop partner closely with EMSL's Technical Leads and scientific staff to design and fabricate instruments. Many of the instrument enhancement and new capability development activities that occur at EMSL are the result of items designed and constructed in EMSL's machine shop.

Enhancements to EMSL's machine shop began in 2004 and were completed in 2005. 'These enhancements included:

- Adding approximately 600 cubic feet of floor space (from a vacated adjacent user shop)

- Acquiring three major pieces of excess machining equipment, with an estimated value of $\$ 200 \mathrm{~K}$, from closed facilities in the Hanford Site's 300 Area

- Upgrading the ventilation system to accommodate the exhaust from all machine shop equipment, providing a cleaner working environment.

'Ihe original EMSL machine shop was designed to accommodate one full-time machinist. However, by 2004 the demands for machining soon increased to the need for seven full-time staff members. At that time, EMSL employed four full-time machinists who were located in the EMSL machine shop and sent out three full-time machinists to work at other PNNL shops. When sending machinists to other PNNL shops, EMSL staff and users lost the one-on-one working relationship with the machinist, resulting in a lower-quality and noncost-effective product. 
The new enhancements to EMSL's machine shop are allowing the user facility to add a fifth machinist to its staff, resulting in greater use of the EMSL model on its projects.

\section{Radiological Work}

EMSL anticipates increased emphasis and need for performing low-level radiological experiments for the DOE Office of Biological and Environmental Research's Natural and Accelerated Bioremediation and Environmental Management Science programs, the principal programmatic drivers for EMSL's Biogeochemistry Scientific Grand Challenge. No radiologicalfacilities are currently available in EMSL for dispersible radiological samples.

Interfacial molecular science is a rapidly growing area that has contributed enormously to the understanding of the molecular processes that can control the geochemistry and biogeochemistry of natural systems. In this regard, it has the same potential to contribute to the understanding of the fundamental processes controlling the fate and transport of radionuclides in environmental systems and to unraveling the fundamental bonding of actinides and other radionuclides at the interface of manmade materials for selective extraction or materials fabrication. Making such molecular science capabilities available to a wide range of users with an interest in radiochemistry is of importance to EMSL's and DOE's mission.

Currently, all radioactive samples that are brought to EMSL must remain sealed to satisfy PNNL's criteria for volumetric release. Although this requirement does allow certain spectroscopic techniques (e.g., laser fluorescence) to be used to identify the molecular form of radionuclides in certain bulk samples in EMSL, it greatly hinders or completely negates experiments that require in-situ sample preparation or the use of most molecular-levelinterfacial surface probes. The sensitivity of these instruments to near-surface conditions (often tens to hundreds of $\AA$ ) means that any attempt to probe a sealed source with such instrumentation only results in probing the sealing material.

Access to specialized equipment for the study of radiological samples has been declining in this country for a long time. 'Thus, establishment of laboratories in EMSL for use by the scientific community in this area will be a key feature. In addition, providing training for the next generation of radiochemists and others interested in nuclear science has long been the subject of numerous symposia and lectures. The radiochemical capacity in EMSL would represent one key DOE capability that would provide these opportunities at minimal cost and still allow contribution of high-quality science to areas of importance to DOE's mission. 


\title{
1.2
}

\section{Highlights that Support EMSL's Science Themes}

\subsection{1 \\ Biogeochemistry and Subsurface Science Electron Microscopy Evaluation of the Role of Dissimilatory Metal-Reducing Bacteria in Biomineralization Pathways}

\author{
AC Dohnalkova, ${ }^{(a)}$ CM Hansel, (b) YA Gorby, ${ }^{(a)}$ and S Fendorf ${ }^{(b)}$ \\ (a) Pacific Northwest National Laboratory, Richland, Washington \\ (b) Stanford University, Stanford, California
}

\section{Figure 1-1}

Bacterially assisted mineral transformation of ferrihydrite-coated sand in advective flow columns. (a) Ferrihydrite at the starting point. (b) After 16 days of reduction, magnetite was the dominant phase. (c) Unstained cross section of secondary minerals surrounding bacterial cell (the membrane is shown by an arrow). (d) Goethite needle-like crystals (Go), magnetite (Ma), nanocrystalline ferrihydrite (F) Scale bar is 200 nanometers.
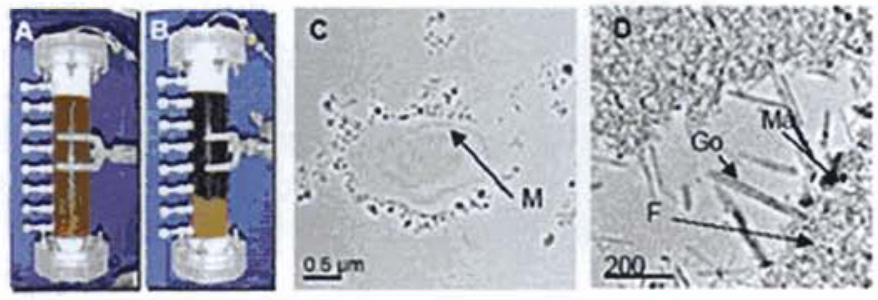

The importance of microorganisms in the biogeochemical cycling of iron is well recognized. Dissimilatory metal-reducing bacteria, which are ubiquitous in soils and aquifers, couple the oxidation of organic matter or $\mathrm{H} 2$ with the reduction of various $\mathrm{Fe}$ (III) oxide phases to obtain energy for growth and function. 'They can also catalyze $\mathrm{Fe}$ (III) reduction under anaerobic conditions, using crystalline and poorly crystalline iron oxides as a terminal electron acceptor.

Microbially induced iron mineral transformations were examined using Shewanella putrefaciens, strain CN32, in an artificial groundwater medium in columns under advective flow conditions (Hansel et al. 2003) (Figures 1-la and b). In this experiment, columns were filled with ferrihydrite-coatedquartz sand inoculated with S. putrefaciens (initial cell density $108 / \mathrm{mL}$ ). Lactate was added 
as an electron donor. Changes in microbial metabolism, aqueous chemistry, and solid-phase distributions were then monitored at time points until termination of the column experiment at 16 days.

Transmission electron microscopy (TEM) and SEM were used for investigating mineral association with bacterial cells, crystal size, morphology, and spatial relationships. A special TEM sample preparation protocol developed at EMSL was used for the accurate preservation of both the biological and mineral portion of the sample. To eliminate exposure of the anaerobic sample to oxygen, the whole embedding procedure, as well as the thin sectioning on an ultramicrotome, was carried out in an anaerobic glove box (95 percent argon, 5 percent hydrogen). Ultrathin sections of the material were studied using a JEOL 2010 TEM operating at $200 \mathrm{kV}$ coupled to an Oxford energy dispersive spectroscopy system. Images were collected and analyzed using a DigitalMicrograph $^{\mathrm{TM}}$ (Gatan), with elected area diffraction patterns evaluated by Desktop Microscopist (Lacuna) software.

Visual changes in the solid-phase within the column were evident: initial orange ferrihydrite started turning brown, and further darkened over the course of the experiment, as it was converted to predominantly goethite and magnetite (Figures 1-1c and d). In addition to spectroscopic methods, the presence of goethite and magnetite was further confirmed by TEM and SEM, and the spatial orientations and size of mineral particles were also determined. Typical needle-like structures of goethite crystals were predominantly associated with the surface of ferrihydrite, but were also found coupled with microbial cell surfaces. In fact, some bacterial cells appeared completely encrusted in goethite, most likely a result of electrostatic attraction between newly precipitated goethite and the microbial surface. Magnetite, on the other hand, was mainly associated with the ferrihydrite surface, and only rarely with the cell surface. Thus, the bacterial cell is only indirectly (by ferrous iron production) responsible for goethite and magnetite formation. Although intracellular precipitation of iron oxides in S. putrefaciens was recently reported (Glasauer et al. 2002), only extracellular precipitation was observed in this experimental setup. Bacteria have the ability to shed the mineral deposits from their outer membranes in order to prevent surface passivation caused by mineral sorption. Bacteria appeared to primarily serve as an $\mathrm{Fe}(\mathrm{II})$ source for the system; secondary mineralization was confirmed as a function of initial $\mathrm{Fe}$ (II) concentrations.

The mechanism of enzymatic reduction is not completely understood, and the accountable protein functions are being intensively investigated using several molecular biology techniques. Current and future studies will include immunogold labeling at the electron microscopy level as a method for determining localization of these proteins.

\section{Citations}

Glasauer S, S Langley, and TJ Beveridge. 2002. "IntracellularIron Minerals in a Dissimilatory Iron-Reducing Bacterium." Science 295(5552):117-119.

Hansel CM, SG Benner,J Neiss, A Dohnalkova, RK Kukkadapu, and S Fendorf. 2003. "Secondary Mineralization Pathways Induced by Dissimilatory Iron Reduction of Ferrihydrite Under Advective Flow." Geochimica et Cosmochimica Acta 67(16):2977-2992. 


\section{Interfacial Chemistry and Catalysis $\mathrm{NO}_{2}$ Adsorption of $\mathrm{BaO} / \mathrm{Al}_{2} \mathrm{O}_{3}$ : The Nature of Nitrate Species}

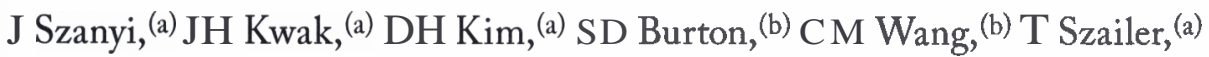 \\ and CHF Peden ${ }^{(a)}$
}

(a) Pacific Northwest National Laboratory

(b) Environmental Molecular Sciences Laboratory

$\mathrm{NO}_{\mathrm{x}}$ storage-reduction catalysis is based on the ability of $\mathrm{BaO}$ to reversibly form $\mathrm{Ba}\left(\mathrm{NO}_{3}\right)_{2}$ under lean engine operating conditions, and release the stored $\mathrm{NO}_{\mathbf{x}}$ in the hydrocarbon rich catalytic cycle. 'Ihe storage of NO, on aluminasupported $\mathrm{BaO}$ has been studied extensively in the past several years using primarily vibrational spectroscopic techniques and temperature programmed desorption (TPD). During thermal decomposition of the $\mathrm{NO}$,-saturated $\mathrm{BaO}$ / A1203 catalysts, two distinct desorption features were commonly seen: at lower temperature $\mathrm{NO} 2$ was observed to evolve only, while at higher temperature the desorption of $\mathrm{NO}$ was accompanied by the evolution of $\mathrm{O}_{2}$. Despite the significant efforts aimed at understanding the NO, storage-release processes, the origin of the two $\mathrm{NO}_{\mathbf{x}}$ desorption features observed in temperature programmed studies has not been addressed. 'The results of this combined Fourier-transform infrared spectrscopy, TPD and a ${ }_{15} \mathrm{~N}$ solid-state NMR study reveals the formation of a unique $\mathrm{Ba}\left(\mathrm{NO}_{3}\right)_{2}$ monolayer strongly interacting with the $\mathrm{Al}_{2} \mathrm{O}_{3}$ support, and upon decomposition releases $\mathrm{NO}_{2}$ only. $\mathrm{BaO}$, in excess of a monolayer, forms bulk-like $\mathrm{Ba}\left(\mathrm{NO}_{3}\right)_{2}$ in $\mathrm{NO} 2$ adsorption and decomposes by releasing $\mathrm{NO}+1 / 2 \mathrm{O}_{2}$. In this study, we have shown for the first time that NMR capabilities can be effectively used to characterize NO2 ions (Janos et al. 2005a).

TPD spectra acquired after $\mathrm{NO}_{2}$ adsorption at $300 \mathrm{~K}$ for $\mathrm{Al}_{2} \mathrm{O}_{3}, 8 \mathrm{wt} \%$, and $20 \mathrm{wt} \% \mathrm{BaO} / \mathrm{Al}_{2} \mathrm{O}_{3}$ catalysts are shown in Figure 1-2 ([NO] and [total NO,] were measured by chemiluminescence; $\left[\mathrm{NO}_{2}\right]=[$ total $\left.\mathrm{NO}]-,[\mathrm{NO}]\right)$. The TPD spectrum from the $\mathrm{NO}_{2}$-saturated $\mathrm{Al}_{2} \mathrm{O}_{3}$ support shows only a broad $\mathrm{NO} 2$ desorption feature between 523 and $873 \mathrm{~K}$. In the TPD spectrum of the $\mathrm{NO}_{2}$-saturated $8 \mathrm{wt} \% \mathrm{BaO} / \mathrm{Al}_{2} \mathrm{O}_{3}$ sample two desorption traces are observed; an NO2 feature with peak desorption rate at $\sim 673 \mathrm{~K}$ and an NO peak with maximum desorption rate at $\sim 775 \mathrm{~K}$ (the desorption of $\mathrm{NO}$ is always accompanied by the evolution of $\mathrm{O}_{2}$ ). 'Ihe shape and the broadness of the $\mathrm{NO}_{2}$ desorption feature indicate that part of this trace is originating in desorption from the $\mathrm{Al}_{2} \mathrm{O}_{3}$ support. Increasing the $\mathrm{BaO}$ coverage to $20 \mathrm{wt} \%$ results in a large increase in the intensity of the NO desorption trace. The NO2 desorption trace shifts to a higher temperature $(\sim 695 \mathrm{~K})$, and the maximum NO

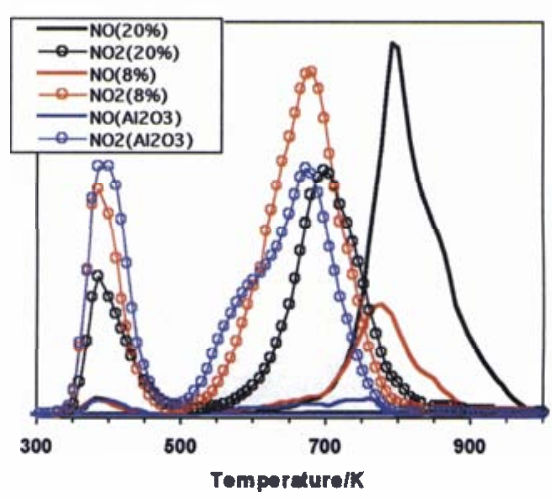

Figure 1-2. TPD spectra form $\mathrm{Al}_{2} \mathrm{O}_{3}, 8 \mathrm{wt} \%$, and $20 \mathrm{wt} \% \mathrm{BaO} /$ $\mathrm{Al}_{2} \mathrm{O}_{3}$ after $\mathrm{NO} 2$ saturation at $300 \mathrm{~K}$. 


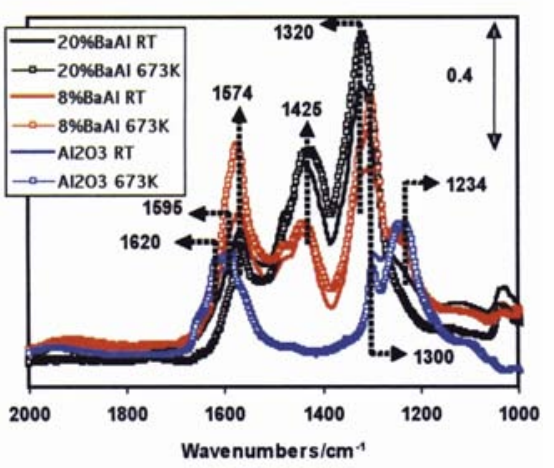

Figure 1-3. Infrared spectra from $\mathrm{Al}_{2} \mathrm{O}_{3}, 8 \mathrm{wt} \%$, and $20 \mathrm{wt} \%$ $\mathrm{BaO} / \mathrm{Al}_{2} \mathrm{O}_{3}$ after $\mathrm{NO} 2$ adsorption at $300 \mathrm{~K}$ (solid lines), and 673K (open circles).

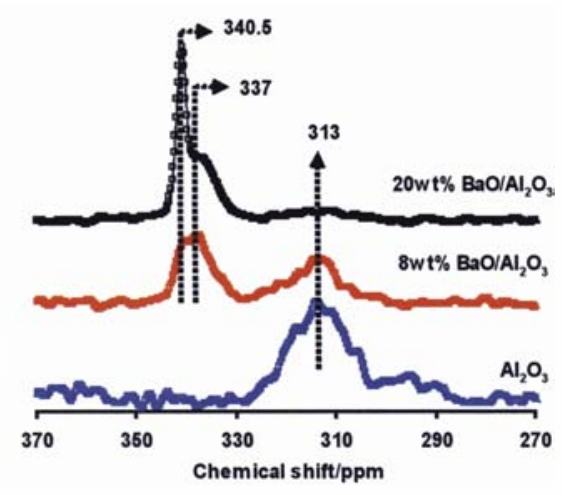

Figure $1-4 .{ }^{15} \mathrm{~N}$ solid state NMR spectra form $\mathrm{Al}_{2} \mathrm{O}_{3}, 8 \mathrm{wt} \%$, and $20 \mathrm{w} t \% \mathrm{BaO} / \mathrm{Al}_{2} \mathrm{O}_{3}$ after $\mathrm{NO} 2$ saturation $673 \mathrm{~K}$. desorption rate is seen at $\sim 795 \mathrm{~K}$. Note the dramatic decrease in the ratio of the amount of evolved $\mathrm{NO}_{2} / \mathrm{NO}$ with increasing $\mathrm{BaO}$ coverage. While only $\mathrm{NO} 2$ is desorbing from the pure $\mathrm{A} 1203$ support, the larger fraction of $\mathrm{NO}$, desorbing from the $20 \mathrm{wt} \% \mathrm{BaO} / \mathrm{Al}_{2} \mathrm{O}_{3}$ is $\mathrm{NO}$. The observed desorption patterns cannot simply be explained by the superposition of the TPD traces from the nitrated $\mathrm{Al}_{2} \mathrm{O}_{3}$ support and $\mathrm{Ba}\left(\mathrm{NO}_{3}\right)_{2}$ formed in $\mathrm{NO}_{2}$ adsorption. As we will show in the discussion of the infrared and NMR results, there is no $\mathrm{Ba}\left(\mathrm{NO}_{3}\right)_{2}$-free $\mathrm{Al}_{2} \mathrm{O}_{3}$ surface present after $\mathrm{NO} 2$ adsorption in the $20 \mathrm{wt} \%$ sample. Thus, all the NO, desorbed in this sample (and most of them in the $8 \mathrm{wt} \%$ sample) is associated with the $\mathrm{BaO}$ storage material. However, the $\mathrm{NO} 2$ desorption feature can not originate from bulk $\mathrm{Ba}\left(\mathrm{NO}_{3}\right)_{2}$ since that decomposes only above $900 \mathrm{~K}$ into $\mathrm{NO}$ and $\mathrm{O}_{2}$ only. We propose that the $\mathrm{NO} 2$ desorption trace from $\mathrm{BaO} / \mathrm{Al}_{2} \mathrm{O}_{3}$ samples originate from adsorbed nitrates on a monolayer of $\mathrm{BaO}$ on $\mathrm{Al}_{2} \mathrm{O}_{3}$, while the $\mathrm{NO}$ is evolving from the decomposition of crystalline $\mathrm{Ba}\left(\mathrm{NO}_{3}\right)_{2}$ particles.

A series of Fourier-transform infrared spectra obtained form $\mathrm{Al}_{2} \mathrm{O}_{3}, 8 \mathrm{wt} \%$, and $20 \mathrm{wt} \% \mathrm{BaO} / \mathrm{Al}_{2} \mathrm{O}_{3}$ after $\mathrm{NO}_{2}$ exposure at $300 \mathrm{~K}$ is displayed in Figure 1-3. Infrared spectra recorded after heating the samples at $673 \mathrm{~K}$ in an $\mathrm{NO}_{2}$ atmosphere for 10 minutes are also shown in the figure. The infrared features observed are in accord with those reported previously for $\mathrm{BaO} / \mathrm{Al}_{2} \mathrm{O}_{3}$. On the $\mathrm{Al}_{2} \mathrm{O}_{3}$ support two types of nitrates are formed in $\mathrm{NO} 2$ adsorption: bridging $\left[1234,1250 \mathrm{~cm}^{-1}\right.$ and $\left.1595,1620 \mathrm{~cm}^{-1}\right]$ and chelating bidentate $\left[1300 \mathrm{~cm}^{-1}\right.$ and $1570 \mathrm{~cm}^{-1}$ ] nitrates. On the $8 \mathrm{wt} \% \mathrm{BaO}$ sample infrared features representing both bidentate (1300 and $1575 \mathrm{~cm}^{-1}$ ) and ionic (1300 and 1420-1480 $\mathrm{cm}^{-1}$ ) nitrates are detected. Note that on the $8 \mathrm{wt} \% \mathrm{BaO}$ sample, nitrates adsorbed onto the $\mathrm{Al}_{2} \mathrm{O}_{3}$ support can clearly be seen (shoulders at 1250 and $1600 \mathrm{~cm}^{-1}$ ) as well. Increasing the $\mathrm{BaO}$ coverage to $20 \mathrm{wt} \%$ results in the nearly complete disappearance of the $\mathrm{Al}_{2} \mathrm{O}_{3}$-related nitrate bands, while the intensities of the ionic nitrate features increase significantly. In particular, there are prominent increases in the relative intensities of the ionic nitrates relative to those of the bidentate nitrates. Heating the alumina support at $673 \mathrm{~K}$ for 10 minutes in $\mathrm{NO}_{2}$ atmosphere does not change the infrared spectrum of the nitrate species. In the $8 \mathrm{wt} \% \mathrm{BaO}$ sample the high temperature heating in $\mathrm{NO} 2$ atmosphere results in a very large increase in the intensities of the bidentate nitrates (bands at 1300 and $1574 \mathrm{~cm}^{-1}$ ), while the doublet feature of ionic nitrates in the 1420 $1480 \mathrm{~cm}^{-1}$ range seem to lose some intensity. In the $20 \mathrm{wt} \% \mathrm{BaO} / \mathrm{Al}_{2} \mathrm{O}_{3}$ the intensities of the ionic nitrates $\left(1320\right.$ and $\left.1420 \mathrm{~cm}^{-1}\right)$ increase as a result of the high temperature anneal in $\mathrm{NO}_{2}$, while that of the bidentate nitrates decrease. The almost complete disappearance of the alumina-related nitrate bands in this sample following the high temperature anneal in $\mathrm{NO}_{2}$ is also noteworthy.

The infrared spectra presented above reveal that in room temperature $\mathrm{NO}_{2}$ adsorption, nitrate species are formed on both the alumina support, and on the $\mathrm{BaO}$ particles. It is also evident that some of the $\mathrm{Al}_{2} \mathrm{O}_{3}$ support surface is free of $\mathrm{BaO}$, even at a relatively high $\mathrm{BaO}$ coverage of $20 \mathrm{wt} \%$, it being more pronounced at lower $\mathrm{BaO}$ coverages. The infrared spectra clearly show that both bidentate (surface) and ionic (bulk) nitrates are formed in both 
$\mathrm{BaO}$-contaning samples, and that the relative intensity of the ionic nitrate peaks increase with $\mathrm{BaO}$ coverage. The infrared results after the $673 \mathrm{~K}$ heating suggest that there is redistribution of the barium-nitrates on the alumina surface at high temperatures. A monolayer barium-nitrate forms on the surface of the $\mathrm{Al}_{2} \mathrm{O}_{3}$ support, resulting in increased intensities of the bidentate nitrates, and a concomitant decrease in the intensities of the ionic nitrates in the $8 \mathrm{wt} \% \mathrm{BaO}$ sample. However, at this low coverage, this wellordered $\mathrm{Ba}$-nitrate film is not covering completely the entire $\mathrm{Al}_{2} \mathrm{O}_{3}$ surface, as alumina-related nitrate bands are still visible. The presence of ionic nitrates indicates that at this $\mathrm{BaO}$ coverage the nitrate layer is not able to access the entire alumina surface. In the $20 \mathrm{wt} \%$ $\mathrm{BaO} / \mathrm{Al}_{2} \mathrm{O}_{3}$ sample, the $\mathrm{Al}_{2} \mathrm{O}_{3}$ surface is almost completely covered with this nitrate monolayer after the $673 \mathrm{~K}$ anneal in $\mathrm{NO}_{2}$ and large barium-nitrate

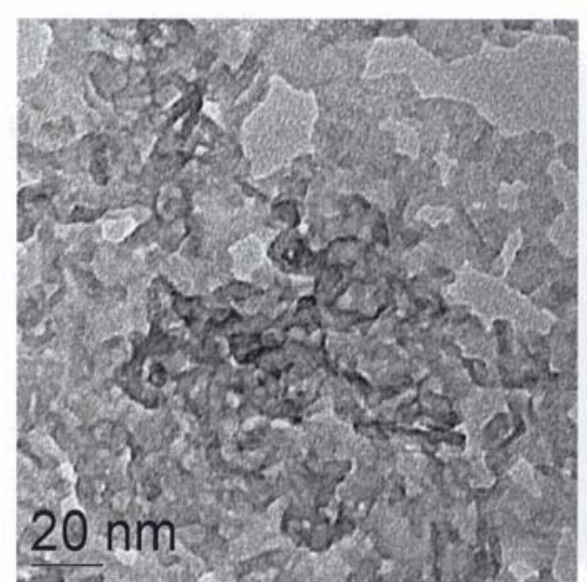
particles grow on top of this film. Note the very good correlation between the intensity ratios of the bidentate/ionic nitrates in the infrared spectra, and those of the $\mathrm{NO}_{2} / \mathrm{NO}$ peaks in the TPD spectra.

${ }^{15} \mathrm{~N}$ solid-state $\mathrm{NMR}$ spectra from the $\mathrm{Al}_{2} \mathrm{O}_{3}, 8 \mathrm{wt} \%$, and $20 \mathrm{wt} \% \mathrm{BaO} / \mathrm{Al}_{2} \mathrm{O}_{3}$ catalysts after ${ }^{15} \mathrm{NO}_{2}$-saturation at $673 \mathrm{~K}$ are displayed in Figure 1-4. On the A1203 support, a very broad NMR feature is seen at $313 \mathrm{ppm}$ chemical shift (relative to the ${ }^{15} \mathrm{NH}_{4} \mathrm{Cl}$ ). Two new peaks appear at 337 and $340.5 \mathrm{ppm}$ chemical shifts on both $\mathrm{BaO} / \mathrm{Al}_{2} \mathrm{O}_{3}$ samples, although with very different relative intensities, and can be assigned to nitrate species. The NMR peak at $337 \mathrm{ppm}$ chemical shift can be assigned to the nitrate monolayer covering the $\mathrm{Al}_{2} \mathrm{O}_{3}$ support, while the 340.5 ppm peak represents crystalline $\mathrm{Ba}\left(\mathrm{NO}_{3}\right)_{2}$ particles. The changes in the ratio of the $337 \mathrm{ppm} / 340.5 \mathrm{ppm}$ peak intensities in the NMR spectra is in line with the increases of the ionic nitrate peaks in the infrared spectra as the $\mathrm{BaO}$ coverage increased form 8 to $20 \mathrm{wt} \%$. It is also consistent with the TPD peak ratios of $\mathrm{NO}_{2} / \mathrm{NO}$.

'The morphology changes during the reaction processes were studied by TEM (Janos et al. 2005b). TEM image, collected with the same resolution, of the A1203 support, and $773 \mathrm{~K}$-calcined $20 \mathrm{wt} \%-\mathrm{BaO} / \mathrm{Al}_{2} \mathrm{O}_{3}$ material is displayed in Fig. 1-5a (left). Energy dispersive spectroscopy analysis of the 20wt\%$\mathrm{BaO} / \mathrm{Al}_{2} \mathrm{O}_{3}$ catalyst confirms the even distribution of the barium-containing phase on the alumina support. The two spots analyzed show practically the same Aluminum/barium atomic ratios (83/17 and 82/18, respectively). TEM images from a $20 \mathrm{wt} \%-\mathrm{BaO} / \mathrm{Al}_{2} \mathrm{O}_{3}$ sample after saturation with $\mathrm{NO}_{2}$ at $673 \mathrm{~K}$ is displayed in Figure 1-5. The formation of $\mathrm{Ba}\left(\mathrm{NO}_{3}\right)_{2}$ crystals during $\mathrm{NO} 2$ uptake is evidenced by the appearance of dark spots in the TEM images. 'This process is further substantiated by the results of energy dispersive spectroscopy analysis on selected spots of this sample. It is interesting to note that we

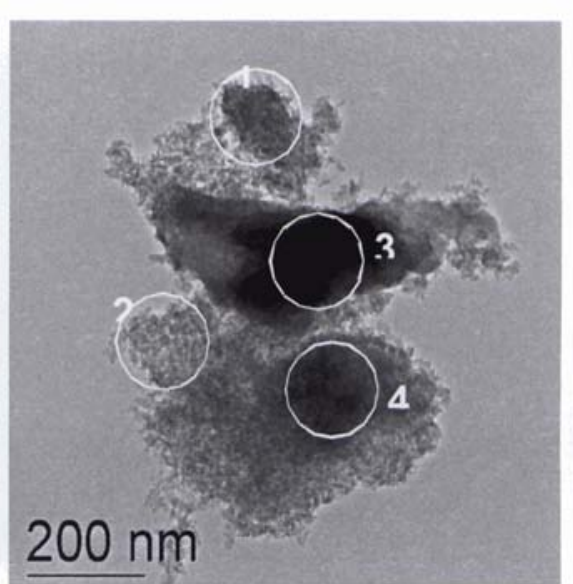

Figure 1-5. TEM images of $20 \mathrm{wt} \% \mathrm{BaO} / \mathrm{Al}_{2} \mathrm{O}_{3}$ after calcinations at $773 \mathrm{~K}$ (left) and after NO2 uptake $673 \mathrm{~K}$ (right). Left: Energy dispersive spectroscopy results: 1 . Barium1 aluminum $=17 / 83 ; 2$. Barium/ aluminum $=18 / 82$. Right: Energy dispersive spectroscopyresults: 1 . Barium/aluminum $=12 / 88$; 2. $12 / 89$; 3. $29 / 71 ; 4$. $7 / 93$ 
detected barium everywhere on the sample, not just at the places where the presence of $\mathrm{Ba}\left(\mathrm{NO}_{3}\right)_{2}$ crystallites are evident from the TEM images.

\section{Citation}

Szanyi J, J H Kwak, D H Kim, SD Burton, and CHF Peden. 2005. " $\mathrm{NO}_{2}$ Adsorption on $\mathrm{BaO} / \mathrm{Al}_{2} \mathrm{O}_{3}$ : 'The Nature of Nitrate Species." Journal of Physical Chemistry B 109(1): 27-29.

Szanyi J, JH Kwak, J Hanson, CM Wang, T Szaler, and CHF Peden. 2005b.

"Changing Morphology of $\mathrm{BaO} / \mathrm{Al}_{2} \mathrm{O}_{3}$ during $\mathrm{NO}_{2}$ Uptake and Release"Journal of Physical Chemistry B 109(15):7339-7344. 


\subsection{3}

\section{Structure/Dynamics of Biomolecules and Biomolecular Complexes Structural Proteomics: Annotating the Genome Using Three-Dimensional Structure}

A Yee, ${ }^{(a)}$ Bin Wu, ${ }^{(a)}$ T A Ramelot, ${ }^{(b)}$ JR Cort, ${ }^{(b)}$ MA Kennedy, ${ }^{(b)}$ and C Arrowsmith ${ }^{(\mathrm{a})}$

(a) University of Toronto, Toronto, Canada

(b) Pacific Northwest National Laboratory, Richland, Washington

The three-dimensional structure of proteins reveals several biochemical properties and functions that escape prediction based only on primary sequence. Structural proteomics is a genome-wide scale structural biology. Our ongoing structural proteomics project uses NMR spectroscopy as one of the methods for obtaining the solution structure of proteins. Our laboratory at the University of Toronto screens and makes samples amenable for structure determination by NMR spectroscopy. We rely heavily on the advanced NMR capabilities at EMSL for collection of triple-resonance NMR experiments. The structures of a yeast protein and two ribosomal proteins from the archaea Methanobacterium thermoautotrophicum have recently been solved, bringing the total to 14 structures solved so far in this collaboration.

\section{Ribosomal Proteins S17E and S28E}

Ribosomes are protein complexes required for protein synthesis in all organisms. S17E and S28E are two of the 28 proteins that make up the $30 \mathrm{~S}$ ribosome in archaea. 'The $\mathrm{S} 28 \mathrm{E}$ is an all $\beta$ strand protein, while the $\mathrm{S} 17 \mathrm{E}$ is an all-a helical protein (Wu et al. 2003). Both proteins have very high sequence homology to their human ribosomal protein counterpart. The structure of these archaeal proteins provides a good template for modeling human proteins. Figure 1-6 shows the solution structures of S28E, S17E and YHR087W proteins as determined by NMR spectroscopy.

\section{YHR087W}

YHR087W is a protein from Saccharomycescerevisiae, and it was annotated as a hypothetical protein because it has no sequence homologues to any protein of known function. Its three-dimensional structure revealed a resemblance to a domain of the Shwachman-Bodian-Diamond Syndrome protein, a human disease protein. Further structure comparison of the two proteins reveals common features that are likely to be functionally important. Further characterization based on this resemblance is ongoing. 
Wu B, A Yee, A Pineda-Lucena, A Semesi, TA Ramelot, JR Cort, J W Jung, A Edwards, W Lee, M Kennedy, and CH Arrowsmith. 2003. "Solution Structure of Ribosomal Protein S28E from Methanobacterium thermoautotrophicum." Protein Science (12):2831-2837.

\title{
1.2.4
}

\section{Biochemical Pathways Accurate Identification of Peptides Using Tandem Mass Spectrometry}

\author{
KD Jarman, (a) W R Cannon, (a) K H Jarman, (a) A Heredia-Langner, (a) \\ T Malard, ${ }^{(a)}$ and DJ Baxter ${ }^{(b)}$ \\ (a) Pacific Northwest National Laboratory, Richland, Washington \\ (b) W.R. Wiley Environmental Molecular Sciences Laboratory, Richland, \\ Washington
}

In recent years, gene sequences have been completed for a wide array of organism types - from single-cell bacteria to human. 'These genomes are the culmination of world-wide collaborative efforts among academic institutions, private industry, and government agencies. The net result has been the generation of a historically unparalleled amount of publicly available data for organism gene sequences. 'This information resource continues to grow as the sequences of new organisms are completed. Putting this huge information resource to practical use has brought the next layer of knowledge-proteomicsincreasingly to the forefront of biology.

Proteomics is the study of proteins in a whole organism under a given set of conditions. Proteins - the molecular "building blocks" in biological systemsperform any number of special functions, allowing cells to live, grow, and react to their surroundings. Proteins are made by joining amino acid residues (smaller molecular units) end-to-end in a particular order that is determined by gene sequence. After proteins reach their final or functional form, they are ready to interact with other proteins, substances inside or outside cells, or genes. Because of their central role in almost all of the processes required to sustain life, understanding protein behavior is a key aspect of understanding any organism's behavior.

To keep pace with the growing demand for proteomic data, high-throughput methodologies have been developed which provide a "snapshot" of all the protein contents of a biological sample. One such method is known as tandem mass spectrometry-or MS/MS. Proteins from biological samples being analyzed by MS/MS are first digested with trypsin to produce smaller fragments (peptides), 
which are then sorted by liquid chromatography elution or some other method. These smaller fragments are then sent to the first stage of the MS/MS process, in which the charge-to-mass ratio of the fragment is determined. In the second phase of MS/MS, the population of identical peptides is broken with highenergy beams, resulting in collection of small fragments representing the various ways the peptide can be broken. 'These fragments are sent through a second mass spectrometry stage, and all of their charge-to-mass ratios are obtained in one dataset. The collection of fragment masses can be thought of as a fingerprint - a profile of peak locations which is unique for each peptide. Developing methods for accurately identifying peptides from this fingerprint is the aim of MSCF Computational Grand Challenge 9603 (Principal Investigator: W R Cannon).

Currently, peptides are identified by creating hypothetical fingerprints of known peptides. The list of known peptides can be any available set of protein sequences and identities. 'Then for a given spectrum (experimental fingerprint), the similarity to all known peptides of the correct mass-to-charge ratio is calculated, and statistical methods are used to assess the confidence in each similarity score.

'This peptide identification scheme has been implemented in a software development project, called POLYGRAPH, on two high-performance environments: 1) EMSL's 1960-processor Linux cluster supercomputer, and 2) Altixl, the PNNL Advanced Computing Center's 128-processor machine manufactured by Silicon Graphics. In both environments, the independent nature of individual spectrum scoring has been exploited to achieve near-perfect speedup. On EMSL's supercomputer, early benchmarks of POLYGRAPH, shown in Figure 1-7, scaled well to thousands of processors using a protein database with 88,000 entries. Larger databases, such as the nonredundant protein database (nr), may contain millions of entries and must be distributed for POLYGRAPH to function.

Development of the distributed database version of POLYGRAPH has resulted in novel algorithms for managing this massive amount of data in both shared memory and distributed memory architectures. This represents a significant development for capabilities in the growing field of data-intensive computing, and allows for the solution of previously intractable problem sizes using highperformance machines.

'This EMSL Computational Grand Challenge and Pilot Project produced the paper outlined in Heredia-Langner et al. 2004.

\section{Citation}

Heredia-Langner A, WR Cannon, KD Jarman, and KH Jarman. 2004. "Sequence Optimization as an Alternative to de novo Analysis of Tandem Mass Spectrometry Data." Bioinformatics, 20(14):2296-2304.

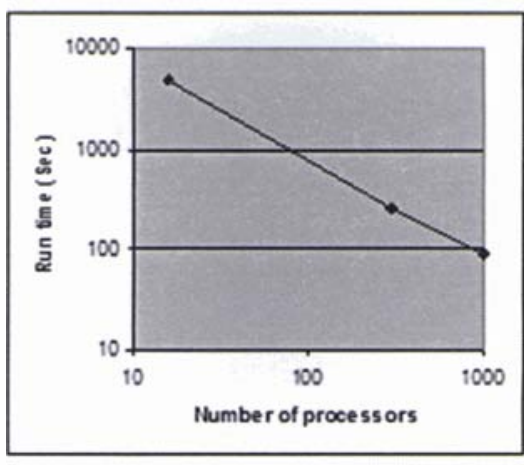

Figure 1-7. Run-time of POLYGRAPH On EMSL'S supercomputer 
samples were transferred back to the microscope, where the same individual particles were analyzed again in order to detect consequential changes in their morphology and chemical composition.

'Ihe dust samples show very different chemical composition and mineralogy with respect to the other dust samples and each other. 'Ihe SEM/EDX data in Figure 1-9 demonstrate this point. Figure 1-9 shows an SEM image and five elemental maps (calcium, magnesium, silicon, nitrogen, and sulfur) of different particles from the coastal Saudi Arabian dust sample. Each of these particles contains high levels of calcium. Two of the particles also contain large amounts of sulfur. 'Ihe particles that contain sulfur (particles labeled a and b) are associated with gypsum. The two other particles (particles labeled $\mathrm{c}$ and d) do not contain significant amounts of sulfur and therefore are carbonate particles. Nitrogen is not observed in any of the particles prior to exposure to nitric acid vapor. After exposure to nitric acid, it is evident from the SEM image that calcium particles that also contain sulfur do not change morphology upon reaction. The nitrogen content of the calcium sulfate particles also does not increase upon reaction, indicating that these particles are not reactive towards nitric acid whereas the carbonate particles increase in nitrogen content.

These results show that by using individual particle analysis that allows for imaging and chemical analysis, dust particle reactivity depends on the mineralogy of individual particles. The carbonate component of the dust is particularly reactive. Importantly, the results of this study indicate substantial differences in individual particle reactivity and therefore clearly show the need to include dust mineralogy in atmospheric chemistry and climate models. This work demonstrates the need to have a detailed assessment of the mineral dust composition and its mineralogy in atmospheric models. These exciting results are published in Krueger et al. 2004.

\section{Citation}

Krueger BJ, VH Grassian,JP Cowin, and A Laskin. 2004. "Heterogeneous Chemistry of Individual Mineral Dust Particles from Different Dust Source Regions: The Importance of Particle Mineralogy." Atmospheric Environment 38(36):6253-6261.

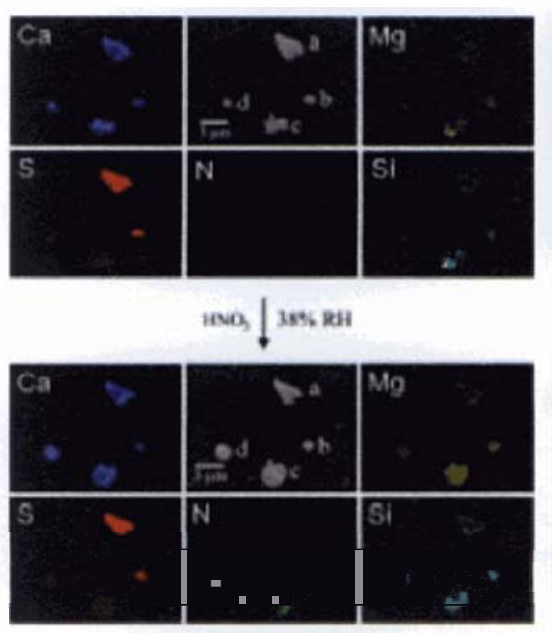

Figure 1-9. SEM image and elemental mapping of individual calcium-containing particles (labeled a-d) of coastal Saudi Arabian dust prior to and after exposure to nitric acid at 38 percent $\mathrm{RH}$. 


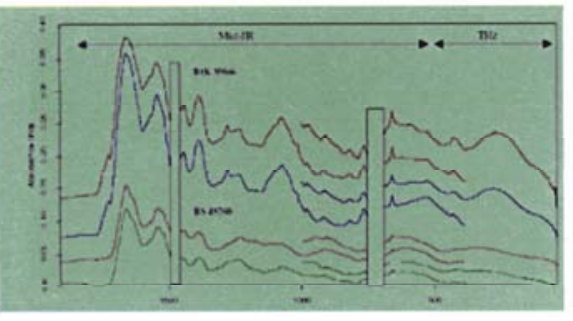

Figure 1-10. Composite midinfrared and $\mathrm{THz}$ spectra of $B$. thuringiensis ssp. kurstaki and $B$. subtilis 49760 from 1800 to $30 \mathrm{~cm}-1$. Those regions where the polyethylene substrate does not transmit have been blocked out for clarity. Spectra were recorded at $2 \mathrm{~cm}-1$ resolution. Spectra have been vertically offset for clarity with the $\mathrm{THz} 3$ spectrum of a sample plotted directly atop the mid-infrared spectrum of the same sample.
1.2 .6

\section{Spectral Signatures and Trace Detection Mid-Infrared Versus Far-Infrared (THz) Relative Intensities of Room-Temperature Bacillus Spores}

TJ Johnson, (a) NB Valentine, (a) and S W Sharpe ${ }^{(\mathrm{a})}$

(a) Pacific Northwest National Laboratory, Richland, Washington

Recently, interest surged in the far-infrared or terahertz $(\mathrm{THz})$ spectral region. An advantage of working at these wavelengths, located between the infrared and millimeter-wave regions, is that many synthetic materials are transparent, and although there are interferences from rotational transitions of water vapor, the transitions are relatively few at the very long wavelengths (i.e., $<50 \mathrm{~cm}^{-1}$ ). Work in the $\mathrm{THz}$ domain recently warranted great interest with emphasis placed on both sensor development (such as those focusing primarily on technologies using pulsed laser sources and gated detectors) as well as signatures recognition-THz spectral signatures have been reported for explosives, nucleotides, DNA/RNA samples, organics, a few gas-phase samples, and pharmaceuticals whose coatings are transparent in the $\mathrm{THz}$ region, thus allowing for mapping within a tablet.

We recently reported identification and discrimination of bacterial spores using mid-infrared technologies. Certain gram-positive bacteria have the ability to form spores, a dormant state that is highly resistant to both chemical and thermal extremes. Using both transmissive and photoacoustic infrared methods, we extended the mid-infrared studies for identifying vegetative bacteria to include bacterial spores. Applying established chemometric methods to our spectra, the data could be reduced to distinguish spores from unknowns, and also distinguish between spores of similar Bacillus species and strains.

The present work compares the relative signal strengths of the mid-infrared to the far-infrared signatures of spores, thus probing the possibility of using $\mathrm{THz}$ signatures for clinical, analytical, or forensic applications (Johnson et al. 2005).

Typical results for bacterial spore absorbance spectra are shown in Figure 1-10 for B. subtilis 49760 and B. thuringiensis subsp. kurstaki. The data above $1900 \mathrm{~cm}^{-1}$ revealed little information other than the well-characterized C-H stretches, and are therefore not discussed here. 'The strongest bands are the protein Amide I and Amide II bands near 1657 and $1541 \mathrm{~cm}^{-1}$, respectively, with nucleic acid and lipid skeletal modes seen at lower frequency. Of greater interest is the lack of spectral signatures seen in the long wavelength regions (wavenumbers $<600 \mathrm{~cm}^{-1}$ ). Each of the mid- and far-infrared spectra was recorded for the same sample on the same substrate. With the exception of a weak $431 \mathrm{~cm}-1$ band, no far-infrared signatures were found that are clearly 
associated with the sample. Although an exact spore count for the sample is not easily ascertained, an estimated spore count was between 108 and 1010.

The present data demonstrate that any $\mathrm{THz}$ signatures associated with the room-temperature spectra of bacterial spores are comparatively weak. Averaged over 30 spectra and comprising five Bacillus species, any $\mathrm{THz}$ signatures are 28.5 times weaker (based on p-p noise) than the $1657 \mathrm{~cm}^{-1}$ Amide I band. 'The lack of $\mathrm{THz}$ peaks makes conjecture as to their (non-) origin difficult. Others have reported DNA/RNA/nucleotide $\mathrm{THz}$ signals, but a spore is a complex containing thousands of chemical species. Smearing of large molecule signatures may play a role, as well as masking by spore-bound water molecules. It may be that the transition dipole moments for such large amplitude motions are fundamentally weak. We anticipate cooling the samples (e.g., 77 or $10 \mathrm{~K}$ ) would increase the intensity by narrowing the linewidths of the $\mathrm{THz}$ bands as has been shown (10-110 $\left.\mathrm{cm}^{-1}\right)$ not only for short peptides, but also long chain retinal and DNA nucleotides. However, for many applications (e.g., first responders), time is critical and precludes using cryogenic methods. Although other species may show stronger signatures, particularly at low temperatures, the present work may suggest a limited utility of the $\mathrm{THz}$ domain for the room temperature detection of spores.

\section{Citation}

Johnson TJ, NB Valentine, and SW Sharpe. 2005. "Mid-Infrared Versus Far-Infrared (THz) Relative Intensities of Room-Temperature Bacillus Spores." Chemical Review Letters 403(1-3):152-157. 


\section{EMSL History and Organizational Relationships}

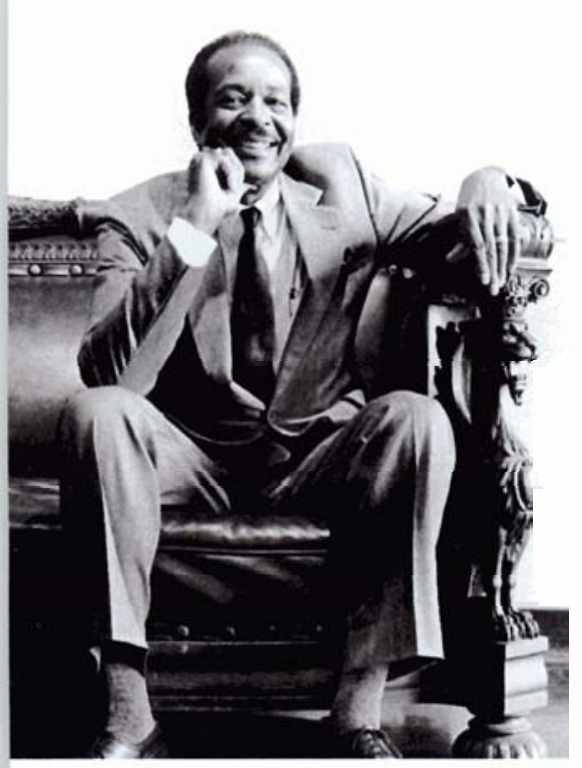

William R. Wilev

\section{1}

\section{History of EMSL}

The genesis of EMSL can be traced to 1986 when PNNL Director William R. Wiley and a handful of his senior managers met to discuss how PNNL could respond to the scientific challenges facing the nation — particularly those related to energy and the environment, which are critically dependant on fundamental advances in chemistry.

The resulting concept was that of a center for molecular science research, located at PNNL, that would bring together theoreticians with expertise in computer modeling of molecular processes with experimentalists from the physical and life sciences. Wiley and others at PNNL, knowing the tremendous advances that were occurring and would continue to occur in the ability of scientists to characterize, manipulate, and create molecules, believed molecular-level research would significantly contribute to solving challenges associated with environmental cleanup, energy efficiency, health, and other important issues. This center would provide PNNL researchers and the nation with a user facility equipped with the most advanced instrumentation available for molecular-level chemistry.

Battelle Memorial Institute, which is headquartered in Columbus, Ohio, and operates PNNL for DOE, shared Wiley's enthusiasm and approved funding to establish the Molecular Sciences Research Center (which was to become EMSL four years later). With these funds, PNNL made plans for developing state-ofthe-art research programs in molecular science and for obtaining the equipment, facilities, and scientists and staff to support these programs.

DOE also recognized EMSL as a natural extension of PNNL's mission. With the facility's location determined and environmental mission established, PNNL was authorized by DOE in October 1993 to proceed with the project. Construction began in July 1994. The facility was dedicated in Wiley's honor in October 1996, a few months after he unexpectedly passed away. Construction was completed in August 1997, and EMSL opened on October 1,1997, for full operations as DOE's newest national scientific user facility. 


\section{2}

\section{DOE and PNNL Organizational Structure}

EMSL is a national user facility supported by the DOE Office of Biological and Environmental Research, an arm of the Office of Science. Within the Office of Biological and Environmental Research, EMSL falls under the responsibility of the Environmental Remediation Sciences Division. Figure 2-1 shows the organizational relationship of EMSL (highlighted in yellow) with DOE.

Figure 2-1. Organizational Relationship of EMSL within the DOE Office of Science.
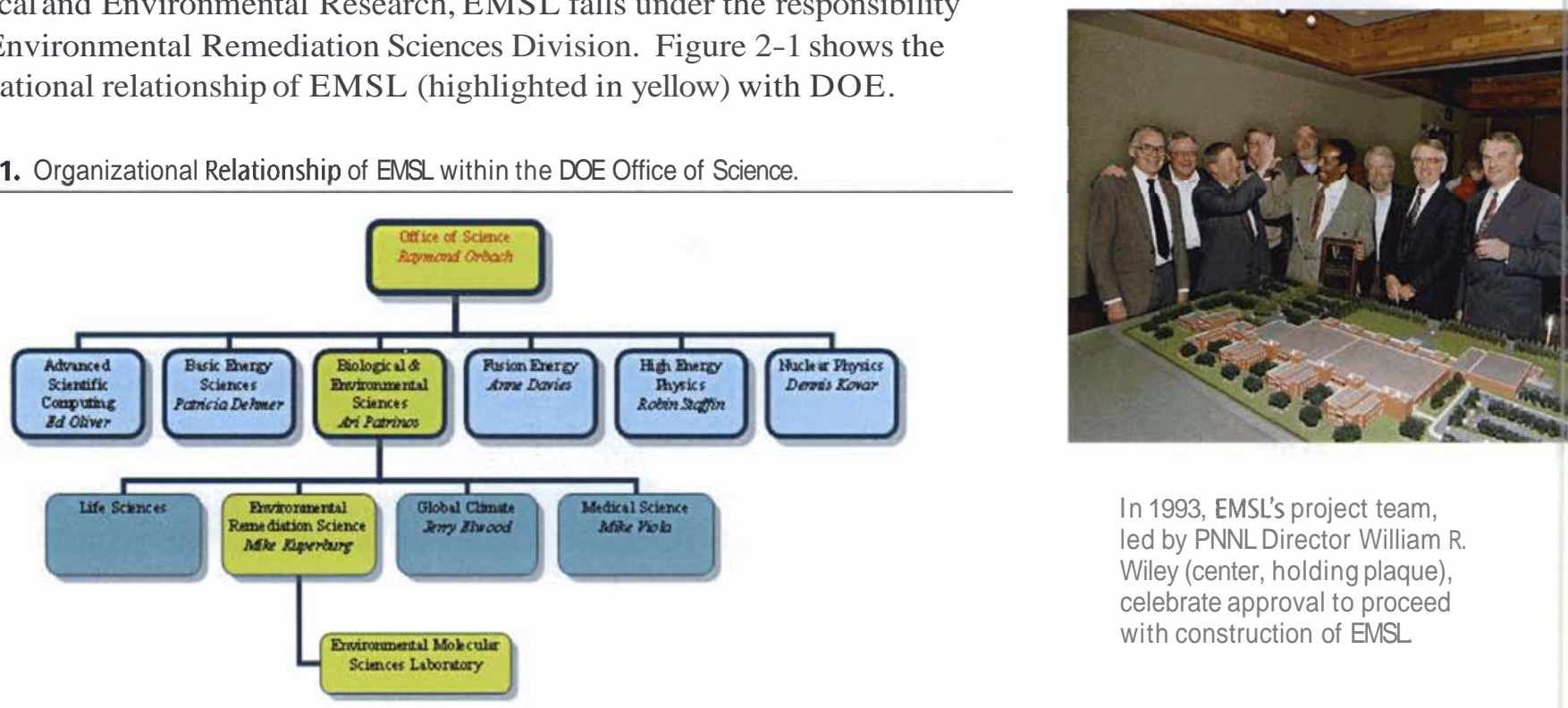

In 1993, EMSL's project team, led by PNNL Director William R. Wiley (center, holding plaque), celebrate approval to proceed with construction of EMSL

EMSL is a stand-alone organization within the organizational structure of PNNL (see Figure 2-2). As shown in the figure, EMSL's Director reports directly to the PNNL Laboratory Director.

Figure 2-2.

Organizational Relationshị, of EMSL within PNNL

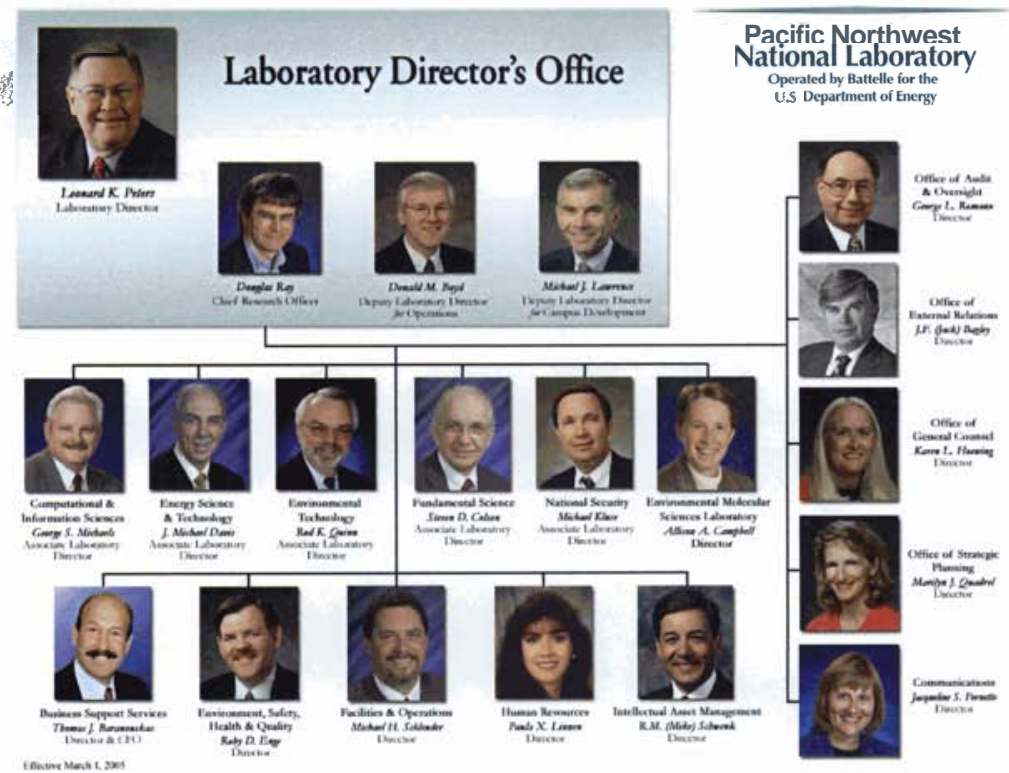


3

\section{EMSL Organizational Structure and Governance}

\section{1}

\section{EMSL Management}

As shown in Figure 3-1, EMSL management is comprised of a Director, an Associate Director for Scientific Experimental Resources, and an Associate Director for Scientific Computational and User Resources. An Operations/ Facilities Manager reports directly to the EMSL Director and is responsible for the safety, space, and operational aspects of EMSL activities. Reporting to the Associate Directors are 10 EMSL Technical Leads, who are responsible for the equipment/resources, staff, and user programs within their assigned groups. Finally, two external advisory committees, the Science Advisory Committee and the User Advisory Committee, are assembled to provide expertise and direction to EMSL management.

Figure 3-1.

EMSL Organizational Structure (Including Advisory Committees)

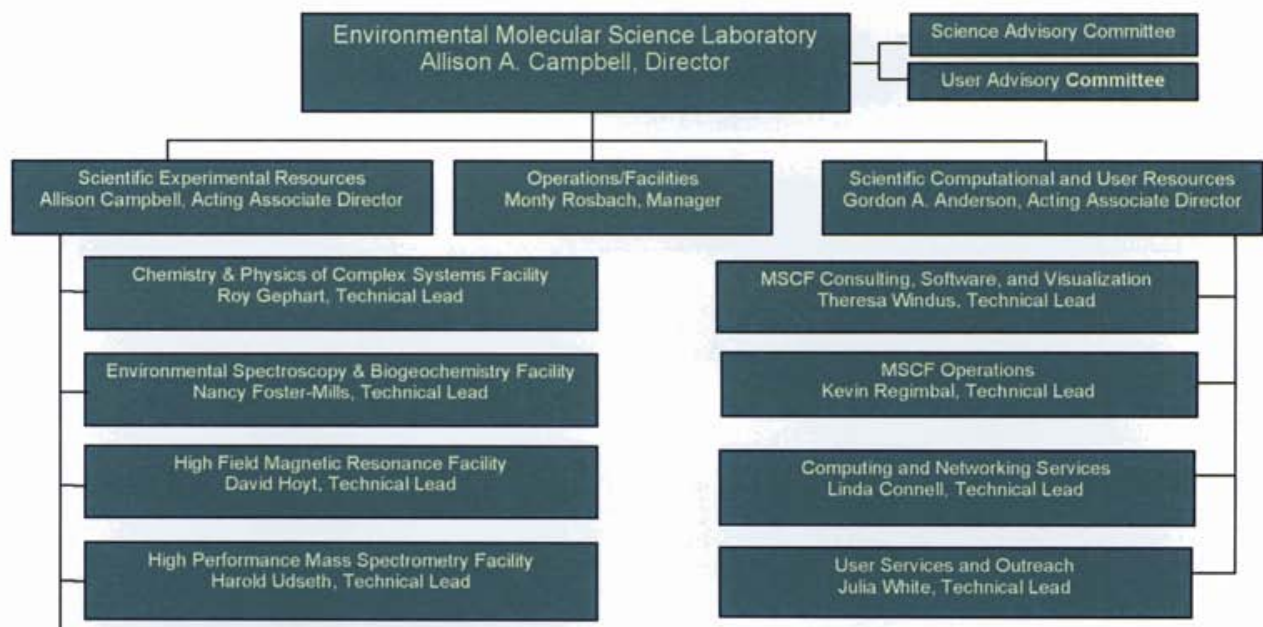




\subsection{1}

\section{EMSL Director}

The primary role of the EMSL Director is to oversee the activities of the Associate Directors, and to work with the advisory committees to design and implement EMSL's strategic plan and vision. 'The EMSL Director is ultimately responsible for all aspects of EMSL's environment, safety and health program as well as stewardship of the user program. Additional details of the EMSL Director's roles, responsibilities, accountabilities, and authorities are provided in Appendix A.

\subsection{2}

\section{EMSL Associate Directors}

EMSL is assigned two Associate Directors: one for Scientific Experimental Resources, and another for Scientific Computational and User Resources. 'These Associate Directors provide direct oversight of EMSL facilities and staff, and are responsible for working with the EMSL Director to implement EMSL's strategy, security, safety, and user programs. Additional details of the EMSL Associate Directors' roles, responsibilities, accountabilities, and authorities are provided in Appendix A.

\subsection{3}

\section{EMSL Operations Manager}

The EMSL Operation Manager is primarily responsible for managing EMSL's environment, safety and health policy and procedures as well as facilitating space and the operational aspects required for staff and users to undertake scientific endeavors. Additional details of the EMSL Operations Manager's roles, responsibilities, accountabilities, and authorities are provided in Appendix A.

\subsection{4}

\section{EMSL Technical Leads}

The EMSL Technical Leads are the frontline managers who are responsible for the daily operations of EMSL's user program and facilities, such as approving/rejecting user proposals, assigning staff to assist users with research, and maintaining facilities. Additional details of the Technical Leads' roles, responsibilities, accountabilities, and authorities are provided in Appendix A. 


\section{EMSL Advisory Committees}

'The Science Advisory Committee and User Advisory Committee are independent bodies charged with providing objective, timely advice to the leadership of EMSL. Both committees report to the EMSL Director. 'The EMSL operations budget funds both advisory committees and is provided by the EMSL Director.

\subsection{1}

\section{Science Advisory Committee}

'The Science Advisory Committee is responsible for the following activities:

- Performing periodic reviews of the scientific impact posed by elements of the EMSL user program. 'These elements include the newly formed CATs, Scientific Grand Challenges, requests for proposals, and unsolicited proposals (open access).

- Providing advice on long-range plans, priorities, and strategies to more effectively address the scientific aspects of EMSL's user program. 'This includes balancing allocation time among the elements of the user program (e.g., CATs, Scientific Grand Challenges) and identifying potential new capabilities or scientific areas of research.

- Providing advice on the appropriate levels of funding and time allocation to develop the aforementioned plans, priorities, and strategies and to help maintain the appropriate balance among competing elements of EMSL.

- Providing advice to EMSL leadership on a variety of scientific and technical aspects, as requested by the EMSL Director. 'The Science Advisory Committee may seek outside input or expand to include $a d b o c$ members who can provide specific expertise in technical areas under discussion.

- Serving as DOE client advocates.

The nature of the goals and purposes of the Science Advisory Committee requires it to be ongoing and dynamic. The committee meets a minimum of once per year, with membership of approximately 20 individuals appointed by the EMSL Director.

Subcommittees of the Science Advisory Committee may be formed to provide recommendations with respect to particular matters related to committee responsibilities. Ad hoc members external to the Science Advisory Committee may be appointed to ensure the competency necessary to conduct the subcommittee's business. 


\subsection{2}

\section{User Advisory Committee}

'The User Advisory Committee is responsible for the following activities:

- Providing advice on how to facilitate the effective use of EMSL. This includes advocacy for user interests as well as recommendations for integrating the various demands on EMSL equipment and staff resources to optimize use and impact.

- Providing advice on priorities and strategies to effectively address the multiple missions of EMSL. 'This includes exploring new paradigms for instrument use and user activity associated with EMSL's broad-based and often unique instrumentation resources.

- Advocating and promoting effective communication between EMSL leadership and the user community to help facilitate mutual understanding in support of achieving maximum impact by EMSL users.

'The User Advisory Committee is comprised of twelve members and includes at least one experienced user in each of the six major research facilities of EMSL. Nominations for committee membership are solicited from EMSL's Technical Leads and Associate Directors. The User Advisory Committee is responsible for recommending to the EMSL Director the appointment of potential new members, and the EMSL Director appoints the committee members and selects the committee chair. In general, committee appointments are made for a term of three years, with the potential for re-appointment to one additional term. Ad boc subcommittees of the User Advisory Committee may be established to focus on specific areas requiring concentrated effort.

In order to secure continuity in committee activity, appointments are staggered so that one-third of the appointments is made at the beginning of each calendar year.

'The User Advisory Committee meets two times a year at approximately sixmonth intervals. Every effort is made to schedule one of these meetings in conjunction with the annual Users' Meeting at EMSL to encourage direct contact between the committee and the broader user community. Additional meetings of the committee or any subcommittees may be scheduled as needed to provide timely advice in support of optimizing EMSL effectiveness. 


\section{EMSL Science and Research Capabilities}

Since opening EMSL, research has focused principally on 1) developing a molecular-level understanding of the physical, chemical, and biological processes that underlie the most critical environmental issues facing DOE and 2 ) developing the computational and experimental tools required to do so. A key attribute of EMSL's research is a close coupling of experimental and computational research to yield insights not attainable by computation or experiment alone.

Research at EMSL is conducted using the resources contained in the six research facilities discussed in this section. EMSL's scientific staff continually push the development of new capabilities as part of their research efforts, which is then translated to the user program. Additionally, EMSL's staff reputation and the scientific challenges being addressed at the user facility attract a significant number of users and collaborators to the facility. This synergy enables EMSL's staff and users to address complex and high-impact scientific issues.

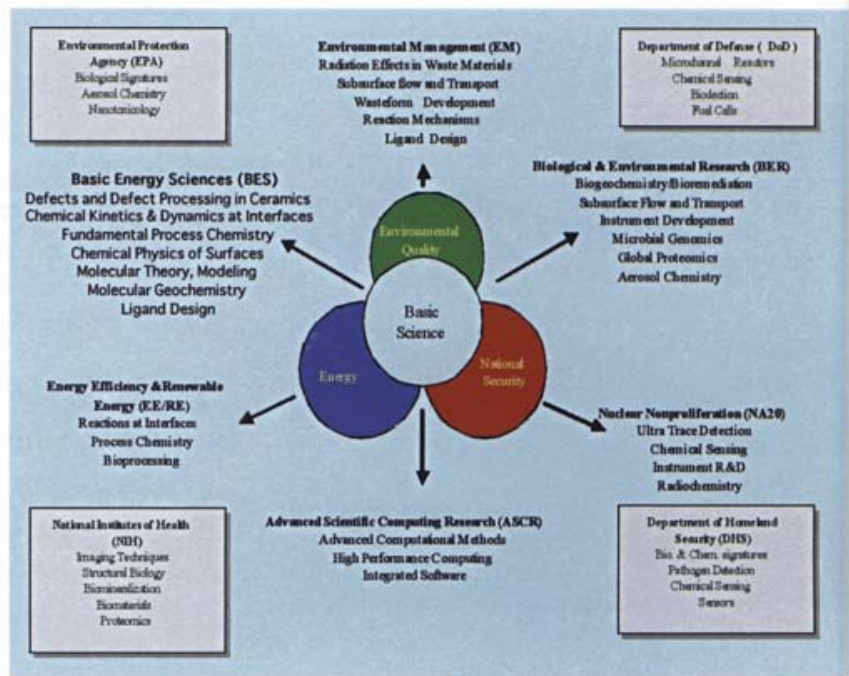

EMSL's research directions link to specific missions within DOE and other government agencies

\section{1}

\section{EMSL Facility Descriptions}

EMSL houses six research facilities that are available, either individually or in combination, for research by users from industry, academia, and other national laboratories. Additionally, EMSL houses an Instrument Development Laboratory and Computing and Networking Services and User Outreach and Support groups. Each facility contains state-of-the-art instrumentation and highly skilled staff who work in an interdisciplinary manner to support user research activities.

This section provides a summary of the research facilities and support groups within EMSL, and highlights some of the critical science performed at each of these facilities. More detailed facility descriptions are provided in Appendix B. In addition, Appendix $C$ provides a list of the cutting-edge scientific instruments that are housed in EMSL and are available for use, and Appendix D provides the resumes and biographies of key facility staff. Finally, Appendix E provides testimonials by facility from past and current users, which support EMSL as a leading national user facility. 


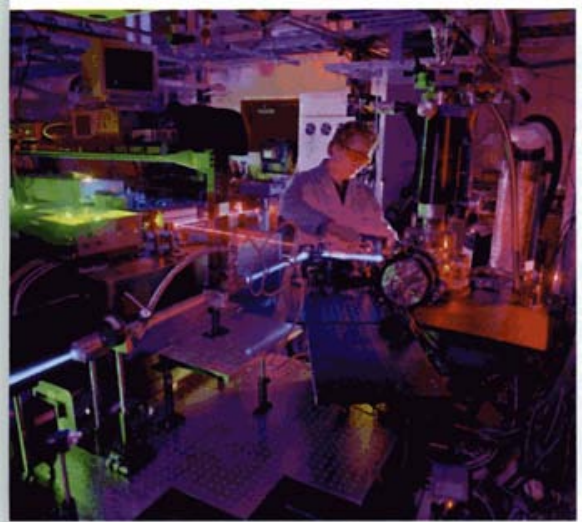

Research at EMSL's Chemistry and Physics of Complex Systems Facility includes laser desorption experiments to investigate the effects of electronic excitation on crystalline materials.

\subsection{1 \\ Chemistry and Physics of Complex Systems Facility}

'Ihe Chemistry and Physics of Complex Systems Facility fosters fundamental research in the natural sciences to provide the basis for new and improved energy technologies and for understanding and mitigating the environmental impacts of energy use and contaminant releases.

\section{Representative Research Activities}

'Ihis facility possesses particular capabilities and expertise that support user research involving preparation and spectroscopic analysis of molecular clusters; high-resolution imaging of biological samples and studies of cellular processes, including DN A damage and repair and low-dose radiation processes; ultra-fast and non linear optical spectroscopies; ultra high-resolution spectroscopy for measurements of electronic and geometric structures and dynamics; surface and interface structure; chemical reaction dynamics and kinetics; ion-molecule traps and storage technology; and specialized chambers, instruments, and models for chemical reactivity and analysis of atmospheric species, including aerosols.

Research at this facility underpins the fundamental understanding of chemical transport and reactivity in the condensed phase. It addresses the underlying uncertainties in thermal and non thermal (i.e., radiation) chemistry, interfacial molecular and ionic transport, and other processes in complex natural and human-made systems related to energy use, environmental remediation, waste management, and understanding biological responses to environmental stresses. One focus is on structures and processes of molecular and nanoscale systems in complex environments, such as condensed phases and interfaces. Research guides the development of new materials and approaches for clean and efficient energy use.

Another central feature of the Chemistry and Physics of Complex Systems Facility is the development of innovative experimental methods with broad applications to research in the natural sciences. Two examples of innovative methods developed by users and staff of this facility include 1) the detection and monitoring of trace atmospheric species, including gaseous and particulate matter, and 2) the study of biological processes important in the environment and health, including imaging of live cells to observe the reaction dynamics of functioning biological systems in real time, advancing the understanding of protein-protein interactions and DNA damage and repair using singlemolecule spectroscopy, and studying cellular responses to low-dose radiation using novel instrumentation 


\section{Research Capabilities}

- High-intensity and high-sensitivity surface chemistry systems

- Systems for thermal and non thermal interfacial chemistry research

- Single-molecule and non linear imaging microscopy systems

- Systems for molecular-scale synthesis and characterization of model materials

- Environmental scanning electron microscope

- Reaction mechanisms at solid, liquid, and gas interfaces

- High-energy processes at environmental interfaces

- Cluster models of the condensed phase

- Ultra sensitive and environmental analysis.

\section{Recent Upgrades}

\section{Pboto-Emission Electron Microscope}

In November 2004, a new photo-emission electron microscope was installed in the Energetic Processes Laboratory of the Chemistry and Physics of Complex Systems Facility. The instrument is capable of imaging nanoscale surface structures by imaging electron emissions induced by ultraviolet and laser light sources. The microscope will be applied to surface science studies of individual nanostructures and catalytic sites on specially designed metal, semi-conducting, and metal-oxide substrates. Since these sites are of nanometer dimension, the highest-resolution instrument available was selected. It uses inherently low-aberration magnetic lens elements to achieve the highest theoretical and practical resolution. "The new instrument will be combined with the ultra fast laser sources at the facility to study femtosecond dynamical processes using unprecedented spatial resolution.

\section{High-Resolution Fourier Transform Spectrometer}

A Bruker 125-high-resolution Fourier transform spectrometer was installed in August 2004 in the High-Resolution Infrared Spectroscopy Laboratory of the Chemistry and Physics of Complex Systems Facility. 'This spectrometer was custom configured for an ultimate spectral resolution of $0.0012 \mathrm{~cm}^{-1}$ and can cover a 10 to $45,000 \mathrm{~cm}^{-1}$ spectral region. The instrument boasts all digital electronics, with each detector equipped with its own analog digital converter for improved performance. The spectrometer can be used with a host of accessories for handling gas, liquid, and solid samples.

\section{Future Direction}

In 2005, the Chemistry and Physics of Complex Systems Facility will focus on performing high-impact science and expanded instrument capability development to support research related to chemical transport and reactivity in the condensed phase, molecular processes, aerosol characterization, and biological imaging. "This research will support the PNNL Biogeochemistry Scientific Grand Challenge as well as the EMSL Scientific Grand Challenge in Membrane Biology. 


\section{Facility Staff}

Roy E. Gephart

Technical Lead

(509) 376-1421

roy.gephart@pnl.gov

Christine C. Montgomery

Administrative Secretary

(509) 376-9867

christine.montgamery@pnl.gov

Stephan E. Barlow

Chief Scientist

(509) 376-9051

se.barlow@pnl.gov

Kenneth M. Beck

Senior Research Scientist

(509) 376-9152

kenneth.beck@pnl.gov

'Thomas A. Blake

Senior Research Scientist

(509) 376-8974

ta.blake@pnl.gov
John L. Daschbach

Senior Research Scientist

(509) 376-2467

john.daschbach@pnl.gov

Gary Holtom

Chief Scientist

(509) 376-5331

gary.holtom@pnl.gov

Martin J. Iedema

Senior Research Scientist

(509) 376-6039

martin.iedema@pnl.gov

Alexander Laskin

Senior Research Scientist (509) 376-8741

alexander.laskin@pnl.gov

\section{Key Staff}

Key staff, in addition to those listed above, include Alan Joly.

\section{Soft-Landing of Peptide Ions on Self- Assembled Monolaver Surfaces}

J Alvarez, (a) RG Cooks, ${ }^{(a)}$ J H Futrell, ${ }^{(b)}$ SE Barlow, ${ }^{(c)}$ and J Laskin ${ }^{(b)}$

(a) Purdue University, West Lafayette, Indiana

(b) Pacific Northwest National Laboratory, Richland, Washington

(c) W.R. Wiley Environmental Molecular Sciences Laboratory, Richland, Washington

Soft-landing of ions on surfaces (deposition of intact ions) is a process that occurs in collision of low-energy ions $(<10 \mathrm{eV})$ with carefully selected semiconductive surfaces. Soft-landing experiments have been reported for small closed-shellions (Miller et al. 1997), oligo-nucleotides (Feng et al. 1999), and, most recently, for multiply protonated proteins (Ouyang et al. 2003). Most of these experiments utilized self-assembled monolayer (SAM) 
surfaces or liquid surfaces (Wu et al. 2000). SAM surfaces facilitate efficient dissipation of the initial kinetic energy of the ion, prevent ion neutralization by charge reduction or proton transfer, and minimize ion-molecule reactions on the surface.

Mass-selected peptide ions produced by electrospray ionization were deposited onto fluorinated self-assembled monolayer (FSAM) surfaces by soft-landing using a FTICR mass spectrometer specially designed for studying interactions of large ions with surfaces. Analysis of the modified surface was performed in situ by combining $2 \mathrm{keV} \mathrm{Cs}^{+}$SIMS with FTICR detection of the sputtered ions. Regardless of the initial charge state of the precursor ion, the SIMS mass spectra included a singly-protonated peptide ion, peptide fragment ions, and peak characteristic of the surface in all cases. In some experiments, multiplyprotonated peptide ions and $[\mathrm{M}+\mathrm{Au}]^{+}$ions were also observed upon SIMS analysis of modified surfaces.

As previously reported for small organic ions, multiply-charged peptide ions can retain charges (typically converting to singly charged ions) when deposited into selected organic monolayer surfaces, such as SAM surfaces. 'The SAM surfaces are effective in reducing neutralization of the peptide ions by proton transfer. Charge retention of soft-landed ions was found to dramatically increase the ion yields obtained during SIMS analysis. We found that a significant number of soft-landed peptide ions retained their charge on the surface even after its exposure to laboratory air, which allowed us to conduct ex situ characterization of modified surfaces using a TOF-SIMS instrument. 'The comparison of TOF-SIMS spectra obtained from an FS AM surface modified by soft-landing and a surface with the same amount of peptide deposited by electrospray provided further support for the presence of preformed ions on the former surface.

We further explored the effect of the initial kinetic energy of the ion on softlanding by exposing the same surface to the ion beam of varying kinetic energy for the same time duration to eliminate possible variations between different surfaces. 'Ihe buildup of the peptide signal was monitored as a function of time (Figure 4-1). If the kinetic energy had no effect on the amount of peptide that can be deposited onto an FSAM surface, we would expect to obtain a linear increase in signal as a function of time. 'The deviation-from linearity of the plot shown in Figure 4-la suggests that the efficiency of soft-landing decreases with collision energy. Indeed, the soft-landing efficiency obtained from the first derivative of the plot decreases with collision energy as shown in Figure 4-1b. 'Ihis decrease can be rationalized by the corresponding decrease in the Langevin capture cross section shown as a solid line in the figure.

Citations

F e n BB, DS Wunschel, CD Masselon, L Pasa-Tolic, and RD Smith. 1999. "Retrieval of DNA Using Soft-Landing after Mass Analysis by ESI-FTICR for Enzymatic Manipulation." Journal of the American Chemical Society 121:8961-8962.

Miller SA, H Luo, SJ Pachuta, and RG Cooks. 1997. "Soft-Landingof Polyatomic Ions at Fluorinated Self-Assembled Monolayer Surfaces." Science 275(5305):1447-1450.

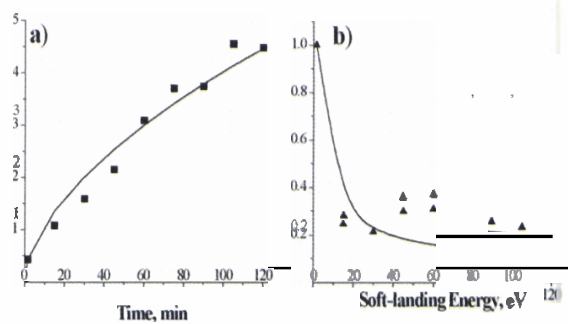

Figure 4-1. a) Cumulative peptide ion abundance (substance P) as a function of soft-landing energy. b) The efficiency of soft-landing. Lines are calculated from the capture cross section at each collision energy. 
from vibrational zeropoint energy differences associated with the frustrated rotational modes of the $\mathrm{H} 20\left(\mathrm{D}_{2} \mathrm{O}\right)$ molecules comprising the water lattice. 'Ihe magnitude of the secondary isotope effect is a sensitive probe of angular anisotropies in the helium-water interaction potential, and the experimental data provide an excellent test of the accuracy of the water/water and helium/water potentials.

\section{Citation}

Daschbach JL, GK Schenter, P Ayotte, RS Smith, and BD Kay. 2004. "Helium Diffusion through $\mathrm{H} 20$ and $\mathrm{D}_{2} \mathrm{O}$ Amorphous Ice: Observation of a Lattice Inverse Isotope Effect." Physical Review Letters 92(19):198306.

\section{Radiation Induced Genomic Instability and Non-Targeted Bystander Effects Induced by the Electron Microbeam}

\author{
M Sowa Resat ${ }^{(a)}$ and WF Morgan ${ }^{(b)}$ \\ (a) W.R. Wiley Environmental Molecular Sciences Laboratory, Richland, \\ Washington \\ (b) University of Maryland, School of Medicine, Baltimore, Maryland
}

Ionizing radiation induces many forms of DNA damage, both directly by energy absorption and indirectly by producing highly reactive free radicals. In recent years, it has become evident that radiation also induces delayed genomic instability, defined as an increased rate of genetic alterations in the genome of progeny of irradiated cells multiple generations after the initial insult. Delayed effects include chromosomal rearrangements and aberrations (chromosomal instability), micronuclei, gene mutations, microsatellite instability, changes in ploidy, and decreased plating efficiency. Many of these effects appear to be mediated by nontargeted effects occurring in cells that were not traversed by an ionizing particle.

Ionizing radiation initiates the instability phenotype either directly by hitting the target cell or indirectly via the secretion of soluble factors or cell-to-cell gap junction-mediated communication from an irradiated cell to a nonirradiated cell (Figure 4-4). Once initiated instability can manifest in the progeny of that cell during clonal expansion and is measured by multiple endpoints. Cell clones showing induced instability can also exhibit persistently elevated levels of reactive oxygen species, which in turn can stimulate changes in gene expression and/or protein/enzyme levels. 'Ihe combination of increased reactive oxygen species and subsequent altered cellular homeostasis provide protracted stimuli perpetuating instability over time. Some unstable clones also generate soluble cytotoxic factors such that media from unstable clones is lethal when transferred to nonirradiated cells. 'Ihis "Death Inducing Effect" results in the induction of DNA double strand cleavage rapidly after transfer to recipient cells, leading to chromosome

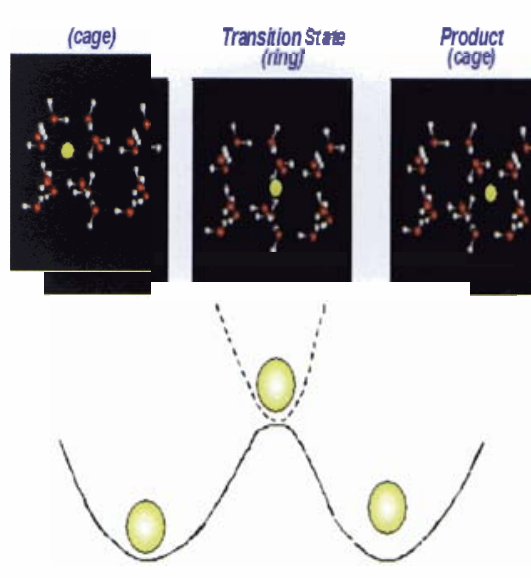

Figure 4-3. Helium diffusion model. Initially the helium atom is trapped in a water cage. In order to diffuse, it must overcome an energetic barrier (transition state). In this model, the transition state is a hexagonal water ring. After passing through the transition state, the helium atom drops down into the adjacent water cage. The lattice inverse isotope effect observed for helium diffusion in $\mathrm{H}_{2} \mathrm{O}$ and $\mathrm{D}_{2} \mathrm{O}$ is the result of a "tight" transition state. 
that few other stable gaseous clusters may be assembled into bulk materials because of cluster-cluster interactions leading to agglomeration. Indeed, no cluster-assembled materials have been synthesized on the basis of stable gaseous clusters other than the fullerenes, despite intensive experimental and theoretical efforts.

Recently, we reported that a 20-atom gold cluster possesses a tetrahedral $\left(\mathrm{T}_{d}\right)$ structure with a remarkably large highest occupied molecular orbital-lowest unoccupied molecular orbital energy gap, suggesting that it would be highly chemically inert and may have novel optical and catalytic properties (Li et al. 2003).

Here, we report the observation of the tetrahedral $\mathrm{Au}_{20}$ cluster in solution, ligated with triphenyl phosphine $\left(\mathrm{PPh}_{3}\right)$ ligands, and its confirmation by theoretical calculations. The current work presents a successful synthetic effort directly guided and motivated by a gas-phase observation, validating the gasphase-to-condensed-phase approach for the discovery of cluster-assembled nanomaterials.

Because of potential cluster-cluster agglomeration, $\mathrm{Au}_{20}$ must also be protected by ligands in order to be used as a building block for cluster-assembled materials. To maintain the unique structural and electronic properties of the $\mathrm{T}_{\mathrm{d}} \mathrm{Au}_{20}$, the ligands must be carefully chosen. 'Thiol ligands strongly interact chemically with gold and would alter the electronic structure of the $T_{d} A_{20}$. Preliminary theoretical calculations revealed that the $\mathrm{Au}_{20}\left(\mathrm{PR}_{3}\right)_{4}(\mathrm{R}=\mathrm{H}, \mathrm{Ph})$ complexes indeed possess high stability. 'The high-resolution transmission electron microscopy (HRTEM) image in Figure 4-6 shows that the soluble samples contained gold nanoparticles with diameters as large as $3 \mathrm{~nm}$, but the majority of the particles have diameters of less than $1 \mathrm{~nm}$. We further characterized the sample using a high-resolution FTICR mass spectrometer, which was accurately calibrated and equipped with an electrospray ionization source. A careful examination of the mass spectrum revealed doubly charged ions corresponding to $\mathrm{Au}_{20}$ clusters with eight and seven $\mathrm{PPh}_{3}$ ligands. To obtain structural information for the $\mathrm{Au}_{20}\left(\mathrm{PPh}_{3}\right)_{8}{ }^{2+}$ cluster, we conducted collision-induced dissociation experiments in the FTICR cell. 'These results, shown in Figure 4-7, suggest that the tetrahedral core of $\mathrm{Au}_{20}$ is intact in the $\mathrm{PPh}_{3}$-coordinated clusters. "This is consistent with our initial expectation and calculation that the four apex sites of $\mathrm{Au}_{20}$ are the most reactive sites that bind strongly to the four $\mathrm{PPh}_{3}$ ligands.

'The current experimental and theoretical results suggest that $T_{d} A_{20}$ clusters coordinated with phosphine ligands may be obtained in bulk quantity. It is expected that by increasing the size of the ligands, one can synthesize the $T_{d}$ $\mathrm{Au}_{20}$ clusters with only the four apex sites coordinated. 'These clusters may be promising catalysts with the highest surface area and well-defined surface sites.

These exciting results are featured in and on a recent cover of theJournal $\oint$ Pysical Chemistry B (Figure 4-8).

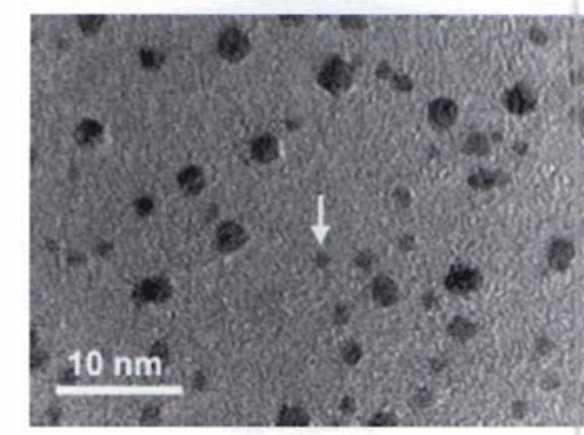

Figure 4-6. HRTEM image of the synthesized gold-phosphine nanoparticles. The arrow points to one possible $\mathrm{Au}_{20}$ cluster.

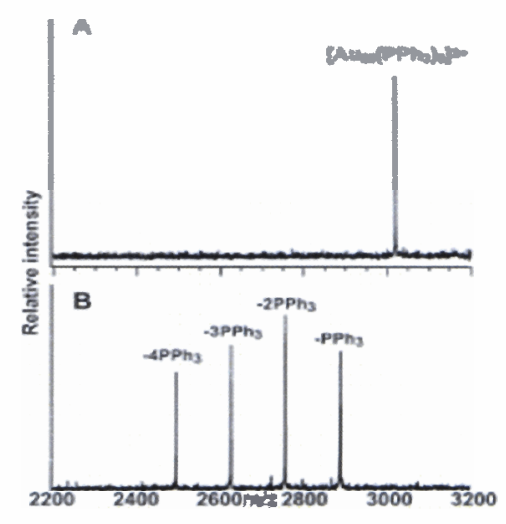

Figure 4-7. Collision-induced dissociation of $\mathrm{Au}_{20}\left(\mathrm{PPh}_{3}\right)_{8}{ }^{2+}$. 


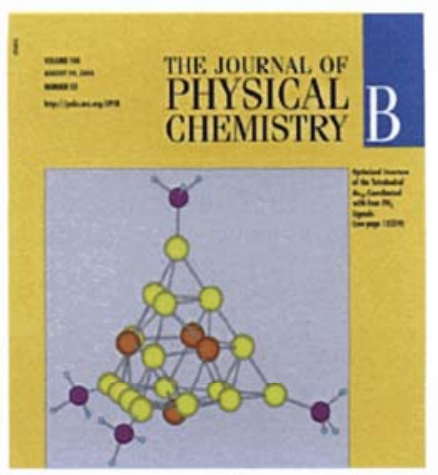

Figure 4-8. This research was featured on the cover of the journal of Physical Chemistry $B$.

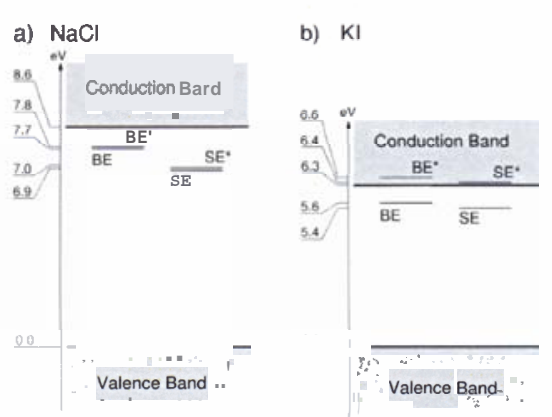

Figure 4-9. Schematic diagram of bulk electronic bands a d bulk and surface exciton states for a) $\mathrm{NaCl}$ and b) KI. SE and SE* are surface exciton states with $\mathrm{J}=$ $3 / 2$ and $1 / 2$, respectively. BE and $\mathrm{BE}^{*}$ are bulk exciton states.

\section{Citations}

Li J, X Li, HJ Zhai, and LS Wang. 2003. "Au20: A Tetrahedral Cluster." Science 299(5608):864-867.

Zhang HF, M Stender, R Zhang, C Wang, J Li, and LS Wang. 2004. "Toward the Solution Synthesis of the Tetrahedral Au ${ }_{20}$ Cluster." Journal of Physical Chemistry B 108(33):12259-12263.

\section{Laser Control of Product Electronic State: Desorption from Alkali Halides}

KM Beck, ${ }^{(a)}$ A G Joly, ${ }^{(b)}$ N F Dupuis, ${ }^{\left({ }^{c}\right)}$ P Perozzo, ${ }^{(d)}$ WP Hess, ${ }^{(a)}$ PV Sushko, ${ }^{(e)}$ and AL Shluger $(\mathrm{e})$

(a) W.R. Wiley Environmental Molecular Sciences Laboratory, Richland, Washington

(b) Pacific Northwest National Laboratory, Richland, Washington

(c) University of California, Santa Barbara, California

(d) Mary Baldwin College, Staunton, Virginia

(e) University College London, United Kingdom

It has been demonstrated that surface selective laser excitation and ultrasensitive atomic desorption techniques can be used to study the behavior of electronically excited alkali halide surfaces (Hess et al. 2002; Joly et al. 2003). A sequence of photo-induced processes involving the excitation, self-trapping, and decomposition of surface excitons produces surface $F$ centers (halogen vacancies that trap an electron) and desorbed hyperthermal halogen atoms. Further, the decomposition of the surface exciton leads to desorption of a mixture of groundand spin-orbit excited halogen atoms.

Through our experimental and theoretical collaborative effort, we have determined that, if the values of the surface exciton energy and the halogen spin-orbit splitting are known, the halogen atom electronic state distribution and yield could be controlled using tunable laser excitation. For $\mathrm{KCl}$, we found that, by tuning the excitation laser photon energy across the absorption threshold, the relative $\mathrm{Cl}^{*}$ yield could be made to vary from near zero to 80 percent, thus dramatically confirming the exciton model and our laser control approach. A similarly large range also was obtained for $\mathrm{NaCl}$. We achieved these results by determining and exploiting the shift between surface and bulk exciton bands and by using photon energies less than those necessary to produce significant bulk absorption or reaction.

Figure 4-9 is a schematic diagram of bulk and surface energy levels. Significantly, the spin-orbit splitting in alkali chlorides is small (AE $\sim 0.1 \mathrm{eV}$ ) so both the $\Gamma_{3 / 2}$ and $\Gamma_{1 / 2}$ exciton states are located within the lowest absorption 
feature at room temperature (Figure 4-9a). Furthermore, in comparison, the shift between the surface and bulk exciton is large $(>0.5 \mathrm{eV})$. "These two factors constitute the requirements for laser control over the halogen atom electronic state distribution.

'The $\mathrm{Cl}$ and $\mathrm{Cl}^{*}$ velocity profiles, from a $\mathrm{NaCl}(100)$ surface at room temperature irradiated at $7.5 \mathrm{eV}$, are shown in Figure 4-10. To generate the velocity profile, the $\mathrm{C} 1$-atom yield is plotted as a function of the delay between pump and probe lasers. 'The velocity profiles can then be converted to kinetic energy distributions by applying the appropriate Jacobian transform (Figure 4-10, bottom graph) (Beck et al. 2001).

Figure 4-11 (top graph) displays relative spin-orbit yields measured for room temperature $\mathrm{KCl}$, as a function of photon energy, and Figure 4-11 (bottom graph) shows the available data for KI. One can clearly see a strong difference in behavior between the chlorides and the larger $\mathrm{Z}$ halides.

We also have successfully measured the hyperthermal atomic emission from hydrothermally grown $\mathrm{CaCl}_{2}$ crystals and $\mathrm{KBr}$ powders, so these results are not confined to alkali halide single crystals. Our model and technique appear to be as general as hyperthermal emission due to surface exciton decay.

\section{Citations}

Beck KM, AG Joly, and W P Hess. 2001. "Evidence for the Surface Exciton in $\mathrm{KBr}$ Via Laser Desorption." Physical Review B 63(12):125423, 1-6.

Hess WP, AG Joly, DP Gerrity, KM Beck, PV Sushko, and AL Shluger. 2002. "Control of Laser Desorption Using Tunable Single Pulses and Pulse Pairs." Journal of Chemical Pbysics 116(18):8144-8151.

Joly AG, KM Beck, M Henyk, W P Hess, PV Sushko, and AL Shluger. 2003. "Surface Electronic Spectra Detected by Atomic Desorption." Surface Science 544(1):L683-L688.

\section{High-Resolution Infrared Spectroscopy and Molecular Structure of Sulfur Trioxide: The $v_{3}$ and $2 v_{3}$ Bands of ${ }^{32} \mathrm{~S}^{16} \mathrm{O}^{3},{ }^{32} \mathrm{~S}^{18} \mathrm{O}_{3}$, ${ }^{34}{ }^{16} \mathrm{O}_{3}$, and ${ }^{34} \mathrm{~S}^{18} \mathrm{O}_{3}$}

T A Blake, ${ }^{(a)}$ R L Sams, ${ }^{(b)}$ S W Sharpe, ${ }^{(b)}$ A Maki, ${ }^{(c)}$ T Masiello, ${ }^{(d)}$ J Barber, ${ }^{(d)}$ EtH Chrysostom, ${ }^{(d)} \mathrm{N}$ Vulpanovici, (d) JW Nibler, ${ }^{(\mathrm{d})}$ and A Weber ${ }^{(\mathrm{e})}$

(a) W.R. Wiley Environmental Molecular Sciences Laboratory, Richland, Washington

(b) Pacific Northwest National Laboratory, Richland, Washington

(c) Independent Researcher
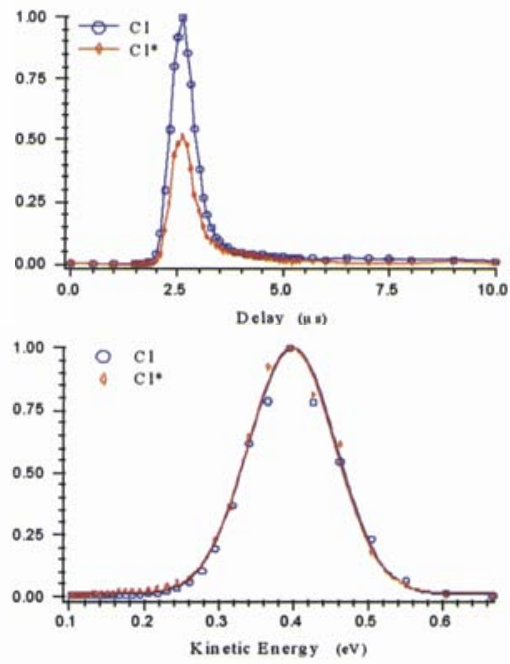

Figure 4-10. Top: Velocity profiles for $\mathrm{Cl}$ and $\mathrm{Cl}^{*}$ following irradiation of room temperature $\mathrm{NaCl}$ at 7.5 eV. Bottom: Kinetic energy distributions for $\mathrm{Cl}$ and $\mathrm{Cl}^{*}$.
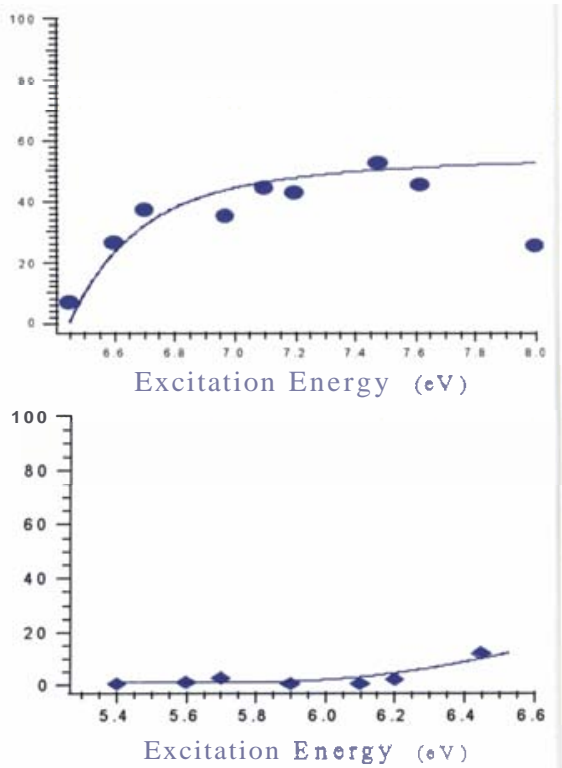

Figure 4-11. The relative $\mathrm{Cl} *$ yield, $\boldsymbol{\Phi C C ^ { * }}=100 \times\left[\mathrm{Cl}^{*}\right] /$ $\left([\mathrm{Cl}]+\left[\mathrm{Cl}^{*}\right]\right)$, for $\mathrm{NaCl}$. Left: The relative hyperthermal I*. Right: The relative $\mathrm{Br}^{*}$ and $\mathrm{I}^{*}$ yield ranges from near zero to $<15$ percent from $\mathrm{KBr}$ and $\mathrm{Kl}$. 


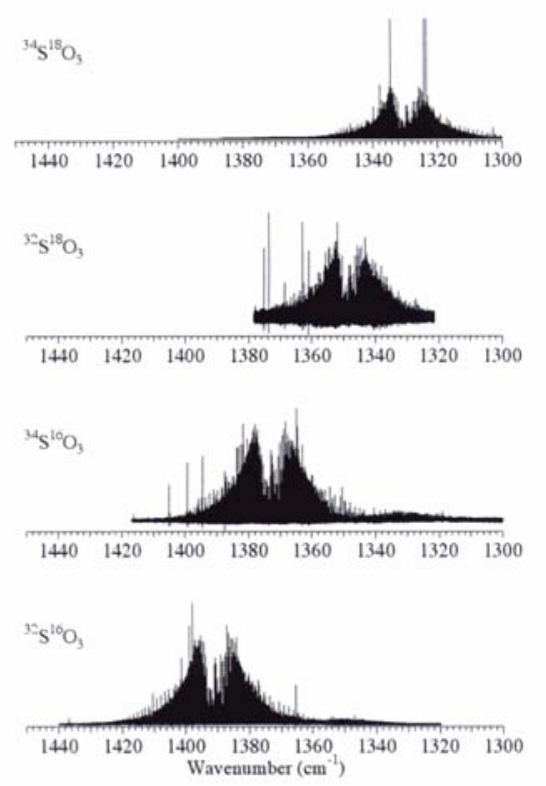

Figure 4-12. High-resolution infrared spectra corresponding to various $v_{3}$ fundamental modes of the ${ }^{34} \mathrm{~s}^{18} \mathrm{O}_{3},{ }^{32} \mathrm{~s}^{18} \mathrm{O}_{3}$ ${ }^{34} \mathrm{~s}^{16} \mathrm{O}_{3}$, and ${ }^{32} \mathrm{~s}^{16} \mathrm{O}_{3}$ molecules used in this detailed rovibrational analysis. (d) Oregon State University, Corvallis, Oregon

(e) National Science Foundation and National Institute of Standards and Technology, Washington, D.C.

During the past several years, we have been engaged in a comprehensive study of the high-resolution spectroscopy of $\mathrm{SO}_{3}$, including all four symmetric top isotopomers formed by ${ }^{32} \mathrm{~S},{ }^{34} \mathrm{~S},{ }^{16} \mathrm{O}$, and ${ }^{18} \mathrm{O}$ (Chrysostom et al. 2001; Maki et al. 2001; Barber et al. 2002; Barber et al. 2003a; Barber et al. 2003b). The initial work focused on the goal of resolving the rotational fine structure of the $v_{1}$ Q-branch coherent anti-Stoke Raman scattering (CARS) spectrum of ${ }^{32} \mathrm{~S}^{16} \mathrm{O}_{3}$ at an instrumental resolution of $0.001 \mathrm{~cm}^{-1}$ (Chrysostom et al. 2001). 'This attempt was only partially successful, in that the K- but not the J-structure was resolved. More interesting, however, was the observed splitting of the Q-branch. This splitting was initially attributed to a Fermi resonance interaction between the $v_{1}$ and $2 v_{4}$ vibrational levels but, even with the inclusion of the somewhat more remote $2 v_{2}$ level, it was found to be impossible to reproduce the details of the observed spectrum without resorting to more infrared measurements. An analysis of the high-resolution (from $0.0015 \mathrm{~cm}^{-1}$ to $0.0025 \mathrm{~cm}^{-1}$ ) infrared spectrum of the hot band transitions from the $v_{2}$ and $v_{4}$ levels to the states $2 v_{2}$ $(1=0), v_{2}+v_{4}(1= \pm 1)$, and $2 v_{4}(1=0, \pm 2)$ was found to be necessary since these levels strongly interact via Fermi resonance and indirect Coriolis interactions with the $v_{1}$ levels. The molecular parameters that resulted from this work enabled the simulation of the $v_{1}$ CARS spectrum with a near-perfect match with the observed one (Barber et al. 2002). In addition, the high-resolution infrared spectra of the $v_{2}, v_{3}, v_{4}$, and $2 v_{3}$ bands of ${ }^{32} \mathrm{~S}^{16} \mathrm{O}_{3}$ (Maki et al. 2001) have been presented and, most recently, for ${ }^{34} \mathrm{~S}^{16} \mathrm{O}_{3}$ the $v_{2}$ and $v_{4}$ bands have been analyzed (Barber et al. 2003a) and the interaction of combination and overtone levels of $v_{2}$ and $v_{4}$ with the $v_{1}$ state has been evaluated (Barber et al. 2003b).

Our most recent results (Sharpe et al. 2003) present a systematic study of the $v_{3}$ and $2 v_{3}$ infrared bands of the four symmetric top isotopomers ${ }^{32} \mathrm{~S}^{16} \mathrm{O}_{3},{ }^{34} \mathrm{~S}^{16} \mathrm{O}_{3}$, ${ }^{32} \mathrm{~S}^{18} \mathrm{O}_{3}$, and ${ }^{34} \mathrm{~S}^{18} \mathrm{O}_{3}$. The observed $v_{3}$ and $2 v_{3}$ bands are shown in Figures $4-12$ and $4-13$, respectively, for the four isotopomers. An internal coupling between the $\mathrm{I}=0\left(\mathrm{~A}_{1}^{\prime}\right)$ and $l= \pm 2\left(\mathrm{E}^{\prime}\right)$ levels of the $2 v_{3}$ states was observed. This small perturbation results in a level crossing between $|k-\ell|=9$ and 12 , in consequence of which the band origins of the $A_{1}^{\prime}, I=0$ dark states could be determined to a high degree of accuracy. For example, the parent isotopic species, ${ }^{32} \mathrm{~S}^{16} \mathrm{O}_{3}, v_{0}$ (I $= \pm 2)=2777.87142(7) \mathrm{cm}^{-1}$ and $v_{0}(I=0)=2766.405(17) \mathrm{cm}^{-1}$ for the $2 v_{3}$ state and $v_{0}=1391.52025(3) \mathrm{cm}^{-1}$ for the $v_{3}$ state. The paper reports ground- and upper-state rotational, centrifugal distortion, and Coriolis coupling constants for the four isotopomers. The vibrational anharmonicity constants $\left(\gamma_{33}+\gamma^{33 \mathrm{~B}}\right)$, $\left(\gamma^{C_{33}}+\gamma^{33 \mathrm{C}}\right), x_{33}$, and $x^{33}$ are also reported. The spectroscopic constants for the center-of-mass substituted species ${ }^{32} \mathrm{~S}^{16} \mathrm{O}_{3}$ and ${ }^{34} \mathrm{~S}^{16} \mathrm{O}_{3}$ vary only slightly, as do the constants for the ${ }^{32} \mathrm{~S}^{18} \mathrm{O}_{3},{ }^{34} \mathrm{~S}^{18} \mathrm{O}_{3}$ pair. The $\mathrm{S}-\mathrm{O}$ bond lengths for the vibrational ground states of the species ${ }^{32} \mathrm{~S}^{16} \mathrm{O}_{3},{ }^{34} \mathrm{~S}^{16} \mathrm{O}_{3},{ }^{32} \mathrm{~S}^{18} \mathrm{O}_{3}$ and ${ }^{34} \mathrm{~S}^{18} \mathrm{O}_{3}$, are, respectively, 141.981 99(1), 141.979 38(6), 141.972 78(8), and 141.969 93(8) $\mathrm{pm}$, where the uncertainties, given in parentheses, are two standard deviations and refer to the last digits of the associated quantity. 
Using the CARS facility at Oregon State University and the high-resolution Fourier transform infrared spectrometer at EMSL, we have been able to extract extraordinary spectroscopic detail from sulfur trioxide, making this the most thoroughly understood nonlinear polyatomic molecule.

\section{Citations}

Barber J, EtH Chrysostom, T Masiello, JW Nibler, A Maki, A Weber, TA Blake, and RL Sams. 2002. "Analysis of $\boldsymbol{v}_{2}, \boldsymbol{v}_{4}$ Infrared Hot Bands of ${ }^{32} \mathrm{~S}^{16} \mathrm{O}_{3}$ : Resolution of the Puzzle of the $v_{1}$ CARS Spectrum." Journal of Molecular Spectroscopy216(1):105-112.

Barber J, T Masiello, EtH Chrysostom,JW Nibler, A Maki, A Weber, TA Blake, and RL Sams. 2003a. "High Resolution Infrared Studies of the $v_{2}, v_{4}$ Bands of ${ }^{34} \mathrm{~S}^{16} \mathrm{O}_{3}$, Including Both Intensity and Wavenumber Perturbations." Journal of Molecular Spectroscopy 218(2):197-203.

Barber J, EtH Chrysostom, T Masiello,JW Nibler, A Maki, A Weber, TA Blake, and RL Sams. 2003b. "Analysis of $\boldsymbol{v}_{2}, v_{4}$ Infrared Hot Bands and $v_{1}$ CARS Spectrum of 3481603." Journal of Molecular Spectroscopy 218(2):204-212.

Chrysostom EtH, N Vulpanovici, T Masiello,J Barber, J W Nibler, A Weber, A Maki, and TA Blake. 2001. "Coherent Raman and Infrared Studies of Sulfur Trioxide." Journal of Molecular Spectroscopy 210(2):233-239.

Maki A, TA Blake, RL Sams, N Vulpanovici,J Barber, EtH Chrysostom, T Masiello, J W Nibler, and A Weber. 2001. "High-Resolution Infrared Spectra of the $v_{2}, v_{3}, v_{4}$, and $2 v_{3}$ Bands of ${ }^{32} \mathrm{~S}^{16} \mathrm{O}_{3}$." Journal of Molecular Spectroscopy 210(2):240-249.

Sharpe SW, TA Blake, RL Sams, A Maki, T Masiello,J Barber, N Vulpanovici, $\mathrm{JW}$ Nibler, and A Weber. 2003. "The $\boldsymbol{v}_{3}, 2 \boldsymbol{v}_{3}$ Bands of ${ }^{32} \mathrm{~S}^{16} \mathrm{O}_{3},{ }^{32} \mathrm{~S}^{18} \mathrm{O}_{3},{ }^{34} \mathrm{~S}^{16} \mathrm{O}_{3}$, ${ }^{34} \mathrm{~S}^{18} \mathrm{O}_{3}$." Journal of Molecular Spectroscopy 222(2):142-152.

\section{Biological Applications of Imaging Micro- and Nano-Scale Metal Oxides Using Multiphoton Microscopy}

GR Holtom, ${ }^{\left({ }^{(a)}\right.}$ YA Gorby, ${ }^{(b)}$ JS McLean, ${ }^{(b)}$ and Y Zheng ${ }^{(b)}$

(a) W.R. Wiley Environmental Molecular Sciences Laboratory, Richland, Washington

(b) Pacific Northwest National Laboratory, Richland, Washington

Despite the enormous activity involving live-cell imaging using fluorescent materials and the continuing improvements in methods and instrumentation, a number of characteristic problems remain that limit the quality of three-dimensional images in long-duration imaging. "These problems are fundamentally related to the physics of the organic chromophores that are usually employed, and appear in the images as photobleaching or photo-induced damage to the cells. To resolve these problems, we are investigating several

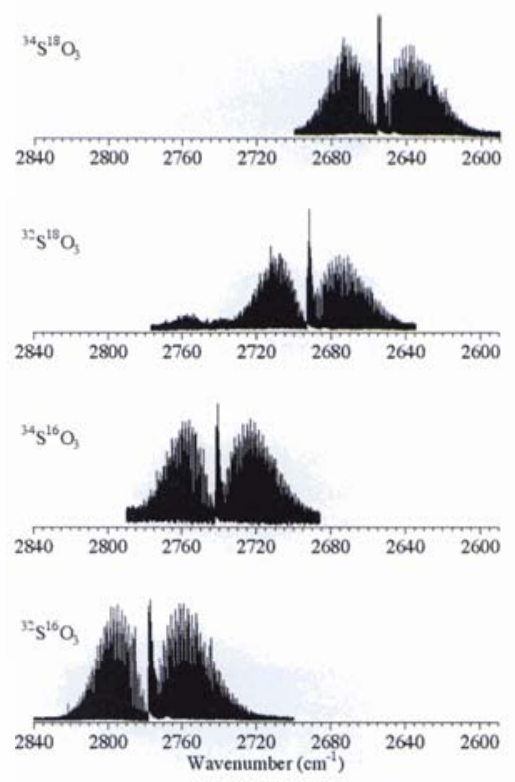

Figure 4-13. High-resolution infrared spectra corresponding to various $2 v_{3}$ first overtone modes of the ${ }^{34} \mathrm{~s}^{18} \mathrm{O}_{3}$, ${ }^{32} \mathrm{~S}^{18} \mathrm{O}_{3},{ }^{44} \mathrm{~S}^{16} \mathrm{O}_{3}$, and ${ }^{32} \mathrm{~S}^{16} \mathrm{O}_{3}$ molecules used in this detailed ro-vibrational analysis. 


\section{Laboratory Studies of Atmospheric Processing of Sea Salt}

B Finlayson-Pitts, ${ }^{(a)}$ A Laskin, ${ }^{(b)}$ D Gaspar, ${ }^{(b)}$ and JP Cowin ${ }^{(c)}$

(a) University of California, Irvine, California

(b) W.R. Wiley Environmental Molecular Sciences Laboratory, Richland, Washington

(c) Pacific Northwest National Laboratory, Richland, Washington

Heterogeneous chemistry of atmospheric aerosol affects both the gas-phase chemical balance of the atmosphere and the physicochemical properties of individual particles. Changes in the properties of individual particles, such as size, shape, composition, and hygroscopicity, will alter aerosol optical properties and thus may impact the Earth's climate.

A team of researchers from EMSL and the University of California at Irvine (Figure 4-16) has examined how sea salt particles overwhelmingly dominate the aerosol composition in the marine boundary layer in remote and coastal urban regions. The ways in which sea salt aerosol impact atmospheric processes such as climate forcing, heterogeneous atmospheric chemistry, and cloud formation are of particular interest in atmospheric science.

Previous studies (Knipping et al. 2000) of the reaction of deliquesced sodium chloride particles with hydroxyl free radicals (a major atmospheric oxidant) showed that the $\mathrm{Cl}_{2}$ generated was far greater than that expected from uptake of $\mathrm{OH}$ into the particles and oxidation of chloride ions in the bulk liquid. A reaction of $\mathrm{OH}$ with $\mathrm{Cl}^{-}$at the air-water interface as a key part of the mechanism was proposed to explain that observation. However, direct measurements to detect changes of the particle composition were not performed in those experiments.

The project at EMSL investigates experimentally the mechanisms of the reactions of sodium chloride with $\mathrm{O}_{3}$ and $\mathrm{OH}$. This work uses EMSL's state-ofthe-art CCSEM/EDX and TOF-SIMS analytical techniques to search for the production of $\mathrm{NaOH}$ in individual particles, which presumably should be formed via the proposed air-water interface mechanism. Initial experiments have been completed and more experiments and analysis is ongoing.

The SEM images in Figure 4-17 are of sodium chloride particles before and after exposure to $\mathrm{OH}$ radicals. Formation of the reaction product recrystallized on the particle surface can be easily seen. It is believed that additional experiments (currently underway) using CCSEM/EDX and TOF-SIMS techniques would allow determination of elemental and molecular composition of the reaction product.

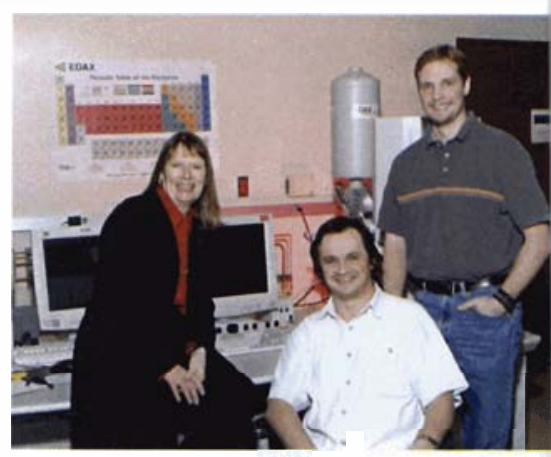

Figure 4-16. Barbara FinlaysonPitts (left), University of California at Irvine, worked with EMSL researchers Alex Laskin (center) and Dan Gaspar (right) to understand the heterogeneous chemistry of sea salt at the single particle level.

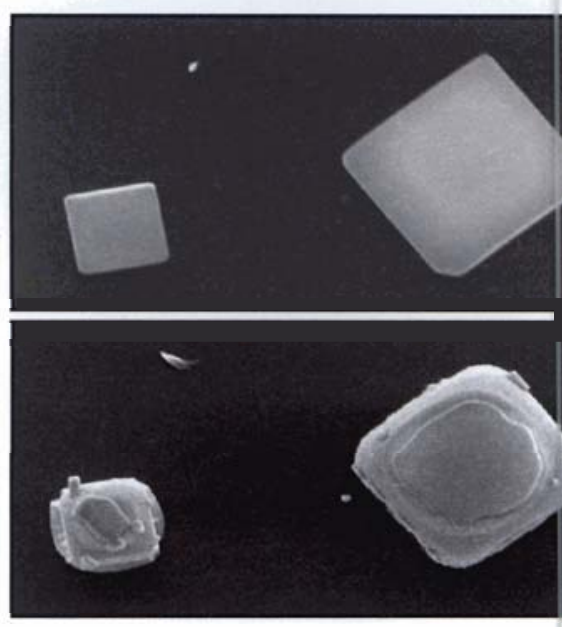

Figure 4-17. SEM images of sodium chloride particles exposed to $\sim 10^{10}$ molecules/ $\mathrm{cm}^{-3} \mathrm{OH}$ at 80 percent relative humidity: before exposure (upper panel) and after exposure (lower panel) 


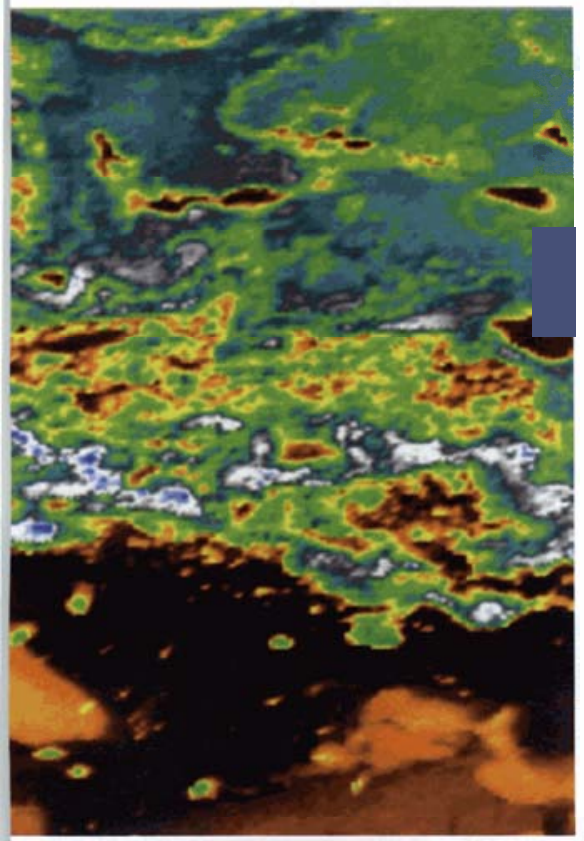

Figure 4-18. The x-ray microprobe technique used here allows the direct fluorescence mapping of $\mathrm{Ca}^{2+}$ constituents at the basalt/caliche interface over the 820- x 350- micron region above. Sample stepping size was maintained at 2.0 microns over this 19-hour scan. Concentrations range from black (min.) to white (max.).

\section{Citations}

Knipping EM, MJ Lakin, KL Foster, P Jungwirth, DJ Tobias, RB Gerber, D Dabdub, and BJ Finlayson-Pitts. 2000. "Experiments and Simulations of Ion-Enhanced Interfacial Chemistry on Aqueous $\mathrm{NaCl}$ Aerosols." Science 288(5464):301-306.

\section{Strontium Migration and Co-precipitation at Columbia Basin Basalt/Caliche Interfacial Regions}

KM Beck, (a) WP Hess, ${ }^{\text {(b) }}$ SM Heald, ${ }^{(b)}$ SH Withers, ${ }^{(c)}$ RJ Peale, ${ }^{\text {(c) }}$ and RJ Reeder ${ }^{(d)}$

(a) W.R. Wiley Environmental Molecular Sciences Laboratory, Richland, Washington

(b) Pacific Northwest National Laboratory, Richland, Washington

(c) University of Central Florida, Orlando, Florida

(d) State University of New York, Stony Brook, New York

Carbonate minerals are components in the soil and vadose zone of several DOE sites, such as the Hanford Site in Richland, Washington. Because they can form rapidly and are highly reactive, carbonates may be extremely effective in attenuating transport of toxic metals and radionuclides by several uptake mechanisms, including co-precipitation and adsorption. In contrast to the sorptive behavior shown by many hydroxide and clay minerals, sorptive uptake of dissolved metals onto calcite usually results in co-precipitation. "This has important implications for the long-term retention of contaminants, since metal species are incorporated into the bulk, rather than solely at the surface of the crystal, and bulk dissolution is necessary for their re-release to fluids.

'Throughout the vadose zone of the Hanford Site, which is dominated by siliciclastic sediments, pedogenic calcification has resulted in the extensive occurrence of carbonate as coatings on grains, as disseminated particles, and as thick and extensive, buried caliche layers (Slate 1996). Where carbonate coatings exist, they are likely to be more important than the substrate grains in controlling uptake of contaminants. Release of highly alkalinity tank waste into the vadose zone, combined with a generally low soil moisture content, is expected to promote periodic precipitation of calcium carbonate, and high $\mathrm{pH}$ conditions should also favor sorption on existing calcite. Preliminary characterization of the caliche underlying a tank waste location at the Hanford Site indicates that ${ }^{90} \mathrm{Sr}^{2+}$ radionuclides are strongly associated with calcite.

A variety of co-precipitation studies have been conducted for divalent metal species with calcite and aragonite, with many corresponding to seawater chemistry conditions. Principal trends have been reviewed by Mucci and 
Morse (1990) and Rimstidt et al. (1998). A significant observation is that transition and post-transition metals (divalent cobalt, zinc, manganese, iron, and cadmium) typically show highly compatible incorporation behavior for calcite (i.e., $\mathrm{K}>1$ ), whereas alkaline earth metals (divalent magnesium, strontium, and barium) are incompatible $(\mathrm{K}<1)$. 'There is, however, a very significant finding among the co-precipitation studies that have assessed the influence of crystallization rate on uptake behavior; specifically, incompatibility diminishes with increasing growth rate (e.g., Lorens 1981; Morse and Bender 1990). Consequently, under conditions of rapid growth, such as might be expected in the soil and vadose zone as a result of periodic wetting and drying, uptake of incompatible (as well as compatible) contaminant species by co-precipitation may be significant for calcite.

'Ihe companion synchrotron-based techniques micro $\mathrm{x}$-ray fluorescence (sometimes called x-ray microprobe) and micro-x-ray absorption spectroscopy, which offer micron-scale element mapping combined with x-ray absorption spectroscopy, are ideally suited for determining spatial heterogeneity of contaminants on natural samples with various levels of information about speciation.

Naturally occurring strontium near the basalt/caliche interfacial region affords an opportunity to investigate how divalent metal leaching and co-precipitation may occur within the caliche layer. Our primary focus is the heterogeneity of contaminant uptake and retention on natural caliche and coatings from Hanford Site samples.

Extensive fluorescence mapping has indicated (as shown in Figures 4-18 and 4-19) that the primary characteristic found in the caliche overlayer is a heterogeneous strontium content with calcium. A co-precipitation process that could account for natural strontium migration and incorporation in "Hanford formation" basalt deposits is consistent with these findings.

Carbonate minerals (particularly calcite) are important components in the soil. In contrast to the sorptive behavior shown by many oxide and clay minerals, sorptive uptake of dissolved metals onto calcite usually results in co-precipitation. Extensive mapping of natural strontium deposits near the basalt/caliche interface via $\mathrm{x}$-ray microprobe indicates hetergeneous calcium and strontium concentrations-"where there is calcium, there is strontium." This implies a coprecipitation mechanism for natural strontium migration and incorporation in caliche. Since metal species are incorporated into the bulk, rather than solely at the surface of the crystal, this has important implications for the long-term retention of contaminants. Uptake of incompatible divalent contaminant species by co-precipitation appears to be significant for calcite.

\section{Citations}

Lorens RB. 1981. “Sr, Cd, Mn, and Co Distribution Coefficients in Calcite as a Function of Calcite Precipitation Rate." Geochimica et Cosmochimica. Acta 45:553-561.

MorseJW, and ML Bender. 1990. "Partition Coefficients in Calcite: Examination of Factors Influencing the Validity of Experimental Results and their Application to Natural Systems." Chemical Geology 82:265-277.

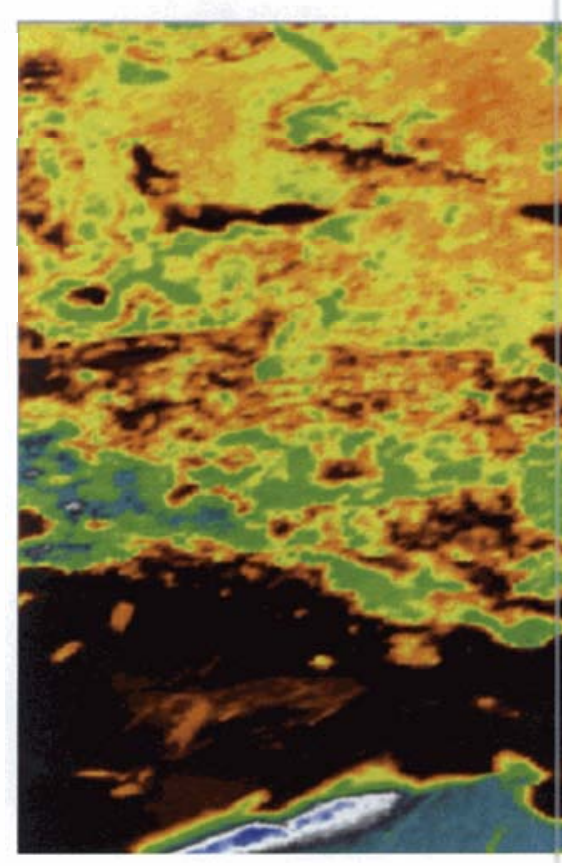

Figure 4-19. The fluorescence signal of $\mathrm{Sr}^{2+}$ was gathered simultaneously over the same region as in Figure 4-18. The heterogeneous nature of the calcium and strontium fluorescence signals apparent in these extensive images may be evidence of co-precipitation. Concentrations range from black (min.) to white (max.). 


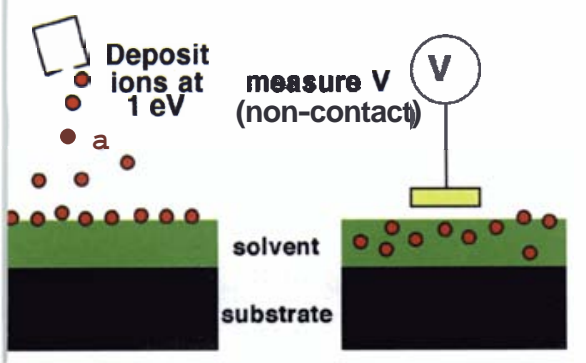

Figure 4-20. Soft-landing ions. At the left, ions are deposited at $1 \mathrm{eV}$ onto prepared cryogenic films of glassy organic, water, or mixed/composite films. At right, thermal and field-induced ion motion is followed via work function changes $\Delta \phi$ as the film is warmed. $\Delta \phi$ scales as the average ion height in the film.

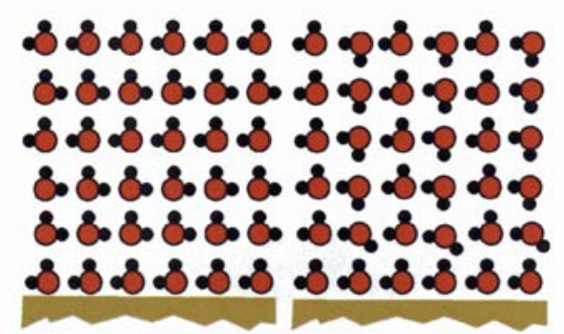

Figure 4-21. Proton ordering at interfaces. The left panel shows a square two-dimensional lattice cartoon of ice on a substrate, where a strong substrate-water interaction propagates water alignment throughout the bulk film. At right is the more realistic situation, where the second-layer defects permit no bulk alignment.
Mucci A, and JW Morse. 1990. "Chemistry o f Low-Temperature Abiotic Calcites: Experimental Studies on Coprecipitation, Stability, and Fractionation." Aquatic Sciences $3: 217-254$

SlateJL. 1996. "Buried Carbonate Paleosols Developed in Pliocene-Pleistocene Deposits of the Pasco Basin, South-Central Washington, U.S.A." Quaternary International34-36:191-196.

RimstidtJD, A Balog, and J Webb. 1998. "Distribution of Trace Elements between Carbonate Minerals and Aqueous Solutions." Geochimica et Cosmochimica Acta, 62(11):1851-1863.

\section{Soft-Landed Ion Study of Water}

JP Cowin, ${ }^{(a)}$ MJ Iedema, ${ }^{(a)} \mathrm{K} \mathrm{Wu},{ }^{(a)}$ and AA Tsekouras ${ }^{(b)}$

(a) William R. Wiley Environmental Molecular Sciences Laboratory, Richland, Washington

(b) University of Athens, Athens, Greece

Water is one of our most fundamentally important materials. We created a unique soft-landing ion source with ionic processes in water as a major goal. One obvious target was proton transport in water ices. In the process of our studies, we also set out to confirm some fundamental properties of water ices (crystalline or amorphous) that standard monographs on water suggest are well understood. We found our studies gave some significant surprises, forcing reassessment of some basic water properties. Results in recent past years include 1) the immobility of protons in crystalline water ice (failure of the Grotthus tunneling mechanism); 2) proton-ordered water ice; 3) field-dependent motion of $\mathrm{D}$-defects in water ice; and 4) dielectric relaxation in amorphous ice. "The newest two results are discussed below.

'The soft-landing ion source is a unique, very-low-energy ion beam that produces mass-selected, aqueous-type molecular ions at $1 \mathrm{eV}$. "This "chemist's ion beam" can gently land ions on or in solvent films to recreate ionic interfaces with unprecedented control, and was built in collaboration with Professor Barney Ellison at the University of Colorado, Boulder.

Applications of the soft-landing ion beam typically follow the approach seen in Figure 4-20. Composite solvent films of thickness from a few to thousands of monolayers thick are first grown a temperatures too low for solvent diffusion to occur. Then ions are deposited, and more solvent can then be added. Upon warming, the ions can move by their attraction to the substrate due to their collective field and ion mobility.

\section{Proton Ordering at the Water-Metal Interface}

Water ice in its familiar form (Ice I, either hexagonal or cubic) is well ordered, both with respect to the oxygen positions and in that all hydrogens are hydrogen-bonded to the oxygen of neighboring molecules, as schematically 
shown in Figure 4-21. But this still allows an infinity of water dipole orientation patterns. 'Ihus, ice is normally proton-disordered, even at $0 \mathrm{~K}$, due to the sluggish ability of the molecules to re-orient. Note from Figure 4-21 that if a strong preference for hydrogen up (or down) occurs at either interface, one would expect this to align the entire growing bulk film, as has been claimed by the groups of Somorjai and Shen (U. Cal. Berkeley), and Toennies (Max Planck, Gottingen). Our recent work examined this issue, and our contradiction of their conclusions was featured in a recent Nature "News and Views" (Bramwell 1999). Our most recent work used careful measurements of the work function change (without ions added) to give the net molecular dipole alignment in each monolayer of the ice from the first through thousands. We confirmed recent theoretical work by Witek and Buch (1999), which concludes that the hydrogen bonding rules are broken in the second water layer (Figure 4-21), permitting equal amounts of up/down hydrogens. Only a feeble alignment tendency of the vacuum interface to orient the hydrogens down persists after the third monolayer ( 2 to 0 percent, depending on $\mathrm{T}$ ).

\section{Amorphous Water: Liquid Above 135 K?}

Much controversy surrounds amorphous water, in the struggle to reconcile diverging properties of supercooled water above $240 \mathrm{~K}$, and amorphous ice from $135 \mathrm{~K}$ through its crystallization near $157 \mathrm{~K}$. The glass temperature of amorphous water has usually been identified as $135 \mathrm{~K}$. We used ion mobility as a probe of any liquid-like nature of amorphous water above $135 \mathrm{~K}$, and also $\mathrm{Kr}$ diffusion in the water. Figure 4-22 shows data for an amorphous water film at one of many conditions of field strength and film thickness. We found no evidence for any ion mobility that would be expected for liquid water above $135 \mathrm{~K} . \mathrm{Kr}$ diffusion also showed no evidence for liquid-like diffusion above 135 $\mathrm{K}$, though it did offer evidence for lateral surface diffusion of water on water, commencing as low as $80 \mathrm{~K}$. We propose a new picture of amorphous water: We identify $228 \mathrm{~K}$ as the true glass temperature of amorphous water, and $135 \mathrm{~K}$ as a proton disordering temperature. This reconciles the properties of supercooled and amorphous water in a simple way.

\section{Citations}

Bramwell ST. 1999. "Condensed Matter Science: Ferroelectric Ice." Nature 397(6716):212-213.

Witek H, and V Buch. 1999. "Structure of Ice Multilayers on Metals." Journal § Chemical Physics 10(6):3168-3175.

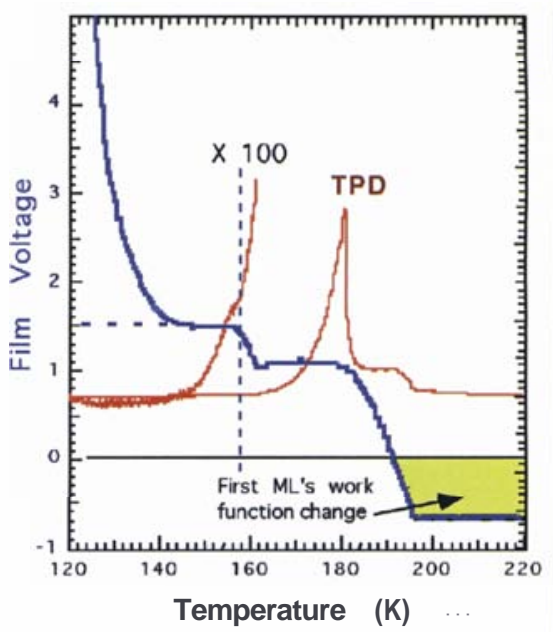

Figure 4-22. Amorphous waterlhydronium ion. 1000 monolayers of amorphous water ice was annealed to 125 $\mathrm{K}$ during hydronium ion dosing. Shown is the subsequent film voltage (descending curve) as $\mathrm{T}$ is increased at $0.2 \mathrm{~K} / \mathrm{s}$. Voltage changes due to dielectric constant changes are seen at 130 and 160 $\mathrm{K}$ and, due to film desorption, near $190 \mathrm{~K}$. The other curve shows water vaporization rate and crystallization, which is complete by $157 \mathrm{~K}$. 
- Mossbauer and EPR spectrometers

- Scanning tunneling and atomic force microscopes

- Multifluid flow/transport cells

- Geochemistry molecular modeling software

- General analytical equipment.

\section{Recent Upgrades}

\section{Pressure Monitoring Flow Cell}

A pressure monitoring flow cell was designed and constructed for use in the Environmental Spectroscopy and Biogeochemisty Facility's Subsurface Flow and Transport Experimental Laboratory. The cell is equipped with several monometer ports to measure the buildup of gas pressure. Initial experiments performed with this new instrument will examine the fate and transport of nitrogen gas produced in situ by indigenous nitrate-reducing organisms.

\section{Mossbauer Laboratory}

'Ihe Mossbauer Laboratory was rearranged to eliminate the possibility of creating magnetic field effects when a future applied-field Mossbauer spectrometer is installed in the proximity of existing spectrometers. To facilitate laboratory rearrangement, two cryostats, along with electronics, sources, computers, and data acquisition systems, were ordered and installed; framing systems for all the Mossbauer spectrometers were designed and installed; and framing and shielding were optimized to allow for installation of permanent radiation shielding. 'Ihe laboratory now contains four Mossbauer spectrometers: one room-temperature instrument, two 4.2-K instruments, and one $10-\mathrm{K}$ instrument. 'Ihe new cryostats allow variable temperature studies to be performed as low as $4.2 \mathrm{~K}$, which is critical to characterizing speciation, mineralogy, and magnetic properties of iron-containing nanoparticles.

\section{Optical Microscope}

$\boldsymbol{A}$ Nikon TE-2000U inverted optical microscope was purchased and integrated with a SpectraPhysics MOPO-730 nanosecond laser system and an ActonResearch MicroSpec 2156i spectrograph fitted with a Roper Scientific Research intensified charge coupled device detector. Currently, this system in the Environmental Spectroscopy Laboratory is being tested for cryogenic fluorescence studies of heavy metal radionuclides, such as uranium, europium, and curium, which are present in contaminated sediments at the Hanford Site in Richland, Washington. 'Ihis optical microscope system can potentially be used to perform time-resolved fluorescence imaging and to subsequently acquire time-resolved fluorescence spectra of specific areas of interest on the fluorescence image.

\section{Ultrafast Laser}

A picosecond diode-pumped Nd:YAG laser was installed at the Environmental Spectroscopy Laboratory. The laser provides an output of 2 watts of 532-nm pulses 


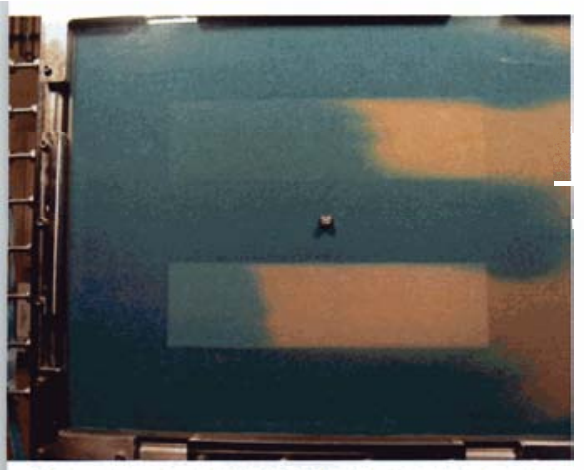

at a repetition rate of $76 \mathrm{M} \mathrm{Hz}$ and a pulse width of $12 \mathrm{psec}$. 'The output can be used to synchronously pump a cavity-dumped dye-laser, providing tunable picosecond pulses in the visible (560 to $760 \mathrm{~nm}$ ) and near-ultraviolet (280 to $380 \mathrm{~nm}$ ) regions through second harmonic generation. This laser combination can be used to measure luminescence lifetimes by coupling with either the Hamamatsu streak camera (providing a time resolution of approximately $10 \mathrm{psec}$ ) or the time-correlated single photon counting system (providing a time resolution of approximately $50 \mathrm{psec}$ ). Current experiments using this laser system involve measuring the fluorescencelifetimes of doped inorganic nanoparticles.

\section{Future Direction}

In 2005, the Environmental Spectroscopy and Biogeochemistry Facility will participate in the new EMSL Biogeochemistry Scientific Grand Challenge, which will involve scientist interaction in the areas of fluorescence spectroscopy; imaging microscopy (atomic force, scanning electron, and transmission electron microscopy); and molecular modeling. In addition, the capabilities of the facility's Mossbauer Laboratory will also be used for research in the

small-scale flow cell. A water soluble dye (green) tracer test was conducted to evaluate the horizontal flow field of the newest intermediate scale flow cell in EMSL's Subsurface Flow and Transport Laboratory. The flow cell was packed with two zones that have lower hydraulic conductivities to demonstrate how the water will wrap around or bypass the regions of lower conductivity. As the solution flowed from left to right, the dye moved faster in courser sand and slower in the two different finer sands.
Biogeochemistry Scientific Grand Challenge as well as EMSL's new CATs. 'The facility plans to develop the following capabilities during the coming year that will help support this Scientific Grand Challenge and CATs.

\section{Laser-Induced Breakdown Spectroscopy/Detection}

This emerging analytical capability offers the prospect of in situ, rapid, highly sensitive, and selective detection and analysis of natural and manmade materials. Such a capability will be developed and used for in situ investigation of nanoparticulates, such as aerosols and nanocolloids, and for possible remote chemical analysis of contaminants in hostile environments. Recently, the development of laser-induced breakdown spectroscopy and detection-based techniques has surged, partly because of the availability of more compact and reliable solid-state lasers, broadband high-resolution spectrometers, and sensitive detectors. The intrinsic similarities noted between laserinduced breakdown spectroscopy and detection and the current laser-induced fluorescence techniques, in combination with the currently available laser systems, detection systems, and expertise in the Environmental Spectroscopy Laboratory, offer an ideal starting point for developing these new and cuttingedge capabilities at EMSL.

\section{Low-Temperature Applied-Field Mossbauer Spectromet y}

The Mossbauer spectrometers located at EMSL are primarily used to identify, characterize, and quantify $\mathrm{Fe}$ (III)-oxides and iron-containing clays in pristine and contaminated soils and sediments. In addition, they are also used to study mineralization associated with dissimilatory bacterial reduction of these $\mathrm{Fe}$ (III) oxides and iron-containing clays. While Mossbauer spectrometers alone are not adequate to fully characterize iron in other systems of interest [e.g., biotransformed $\mathrm{Fe}$ (III) in reduced sediments, such as sediments from the Hanford Site and Oak Ridge Reservation], an applied-field (magnetic) Mossbauer spectrometer coupled with low temperature would provide researchers 
with this ability, along with the capability to characterize iron for application to catalysts, magnetic materials, and nanoparticles.

\section{Small-Scale Flow Cell}

Many experiments in the Environmental Spectroscopy and Biogeochemistry Facility's Subsurface Flow and Transport Experimental Laboratory do not require the use of larger intermediate-scale flow cells. A smaller $(50-\mathrm{cm})$ chlorinated solvent-resistant flow cell would allow researchers to conduct multiple experiments in the time it would take to pack a single larger flow cell. The new flow cell would offer increased flexibility to run an intermediate-scale experiment under multiple conditions more efficiently and would also allow researchers to test ideas and determine experimental parameters before "scaling up" to the larger flow cells.

\section{Facility Staff}

Nancy S. Foster-Mills

Technical Lead

(509) 376-1343

nancy.foster@pnl.gov

Kim Korenkiewicz, Administrative Secretary (509) 373-0765

kim.korenkiewicz@pnl.gov
Ravi Kukkadapu

Senior Research Scientist

(509) 376-3795

ravi.kukkadapu@pnl.gov

Thomas W. Wietsma

ResearchScientist

(509) 376-6588

wietsma@pnl.gov

Paul L. Gassman

Research Scientist

(509) 376-7972

pl.gassman@pnl.gov

\section{Key Staff}

Key staff, besides those listed above, include James E. Amonette, Eric J. Bylaska, Alice Dohnalkova, Andrew R. Felmy, Eugene S. Ilton, Timothy F. Johnson, Alan G. Joly, Chongxuan Liu, Robert Oor, Martinus Oostrom, Odeta Qafoku, Joy D. Rosscup, Kevin M. Rosso, Zheming Wang, and John M. Zachara. 


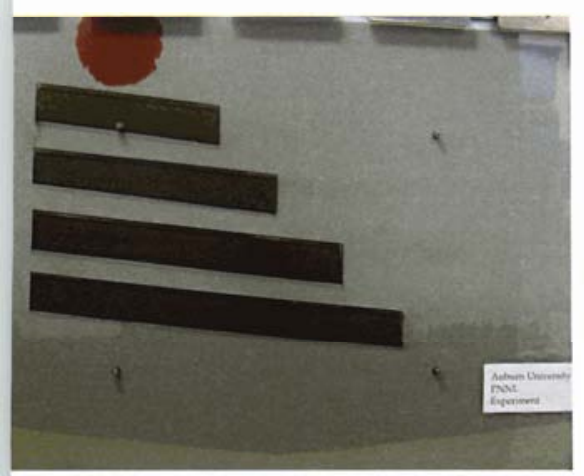

Figure 4-23. The four sloping layers from top to bottom consisted of a fine, a coarse, a fine, and a coarse material. The initial simulated DNAPL spill is the red sphere.

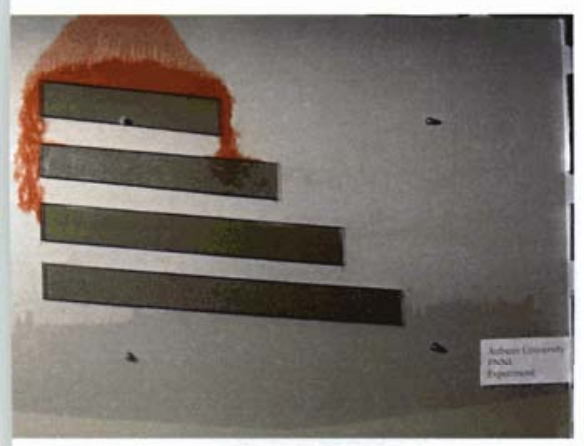

Figure 4-24. Once the DNAPL reached the first fine layer, it started to spread to the left and right and edged over both ends. When the DNAPL hit the course material (second layer) on the right, the solution moved through it.

\section{Removal of DNAPL from a Layered Porous Medium by Means of Vapor Extraction Enhanced by Desiccation and Water Table Reduction}

JH Dane, ${ }^{(a)}$ M Oostrom, ${ }^{(b)}$ and TW Wietsma ${ }^{(c)}$

(a) Auburn University, Auburn, Alabama

(b) Pacific Northwest National Laboratory, Richland, Washington

(c) W.R. Wiley Environmental Molecular Sciences Laboratory, Richland, Washington

Vapor extraction is an in situ soil-cleaning process designed to remove volatile organic compounds from the unsaturated (vadose) zone of soil. Some of the more commonly occurring volatile organic compounds that have contaminated soil and ground water are the dense nonaqueous phase liquids (DNAPLs) trichloroethylene, perchloroethylene, and carbon tetrachloride. "These contaminants can occur in the liquid phase as free, residual, and/or trapped; in the solid phase by means of adsorption, especially to organic matter; in the aqueous phase when dissolved; and/or in the gas phase.

Any remediation treatment stands to benefit from a proper analysis of the liquid and gaseous flow regimes and their fate in the subsurface environment. 'The model must, however, give a true description of all physical, chemical, and biological phenomena. 'Therefore, models must be continuously tested by comparing their results with quantitative data obtained during controlled experiments that mimic real situations. Based on some of our knowledge gaps and desire to enhance our understanding of DNAPL fate in the environment and subsequent cleanup activities, we formulated the following objectives (Oostrom et al. 2004):

- Investigate the infiltration and redistribution of a DNAPL in a variably saturated, layered porous medium by means of an intermediate-scale experiment.

- Remove the non-trapped DNAPL present in the vadose zone by means or wet vapor extraction.

- Remove trapped DNAPL from the vadose zone and that present in the saturated zone by reducing the water table level and employing dry vapor extraction.

- Model the processes of the first three objectives using the computer model STOMP (Subsurface Transport Over Multiple Phases) and compare numerical and experimental results.

To perform the experimental part, four sloped layers were embedded in an otherwise mainly homogeneous porous medium. From top to bottom, these 
layers consisted of a fine, a coarse, a fine, and a coarse material (Figure 4-23). 'The purpose of this layering was to obtain a clear picture of DNAPL behavior at sloped interfaces of materials with different pore sizes and consequently with different hydraulic properties like permeability and displacement pressures. The layers were also thought to affect any remediation efforts. To mimic a real situation, the materials for the layers were obtained from the Hanford Site in Richland, Washington.

Figures 4-23 through 4-26 show a series of four time-elapsed photos taken of the experiment. Initially (Figure 4-23), the DNAPL infiltrated very much as a sphere, similar to the infiltration of water from a point source into dry soil. Once the DNAPL reached the first fine layer, it started to spread to the left and to the right, edging over both ends (Figure 4-24). During the subsequent redistribution, the DNAPL continued to move straight downward on the left side of the flow cell (high ends of the layers), but followed a more intriguing path at the lower ends. 'The first coarse layer, which was unsaturated, accepted the DNAPL after some spreading occurred at the boundary, again indicating that the DNAPL moved as a wetting fluid in the unsaturated zone. Once it had entered the first coarse layer, the DNAPL moved straight downward (Figure 4-24), a result of the diminished capillary action of the bigger pores. As time progressed, the DNAPL on the left side had reached the capillary fringe (saturated zone) and apparently was under enough pressure to displace the water (Figure 4-25). Meanwhile, on the right side of the layers, the free DNAPL had moved through the coarse layer, accumulated on top of the second fine layer, and started to flow down the boundary until it spilled over the edge (Figure 4-25). It then started to spread somewhat on top of and move into the second coarse layer (unsaturated).

As with the first coarse layer, the DNAPL moved subsequently straight through and entered the underlying finer material without much delay. 'The DNAPL again affected the surface tension of water, because the capillary fringe on the right side had also started to collapse (Figure 4-25). The final DNAPL distribution, for which we assumed static equilibrium, is shown in Figure 4-26. It clearly shows the collapse of the capillary fringe, which was attribution to a reduction of the surface tension of water caused by DNAPL diffusion in the liquid phase.

During the infiltration and initial redistribution of a DNAPL in an unsaturated porous medium, its flow is so rapid that the best possible way of documenting its behavior is by obtaining photos (Figure 4-23 through 4-26). However, once static equilibrium of both water and DNAPL had been obtained, gamma radiation attenuation was used to determine liquid saturations at 1150 more or less evenly distributed locations. Additional gamma-radiation scans were obtained to quantify the removal of carbon tetrachloride during the vapor extraction procedures. "The gamma data and analysis of the extracted vapor samples will be modeled using S TOMP to compare numerical and experimental results.

\section{Citation}

Oostrom M,JH Dane, and T W Wietsma. 2004. "Removal of Carbon Tetrachloride from a Layered Porous Medium by Means of Soil Vapor Extraction Enhanced by Desiccation and Water Table Reduction." Vadose Zone Journal (in press).

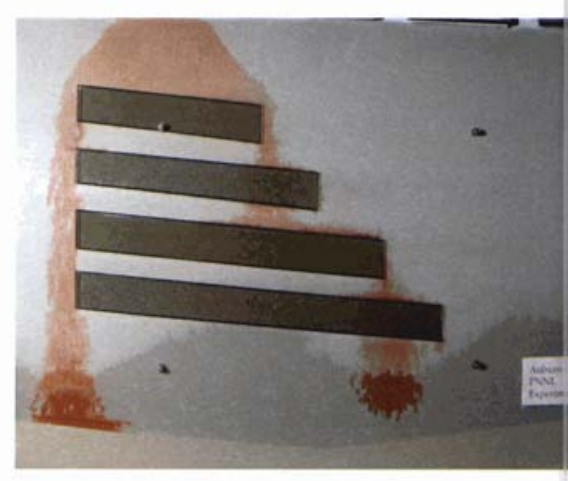

Figure 4-25. On the right side, the DNAPL continues to move downward in a stair step pattern: spreading across the fine material then down, moving through the course material, spreading across the fine material then down, and then moving through the final course material. The collapse of the capillary fringe was evident on both the right and left sides near the DNAPL.

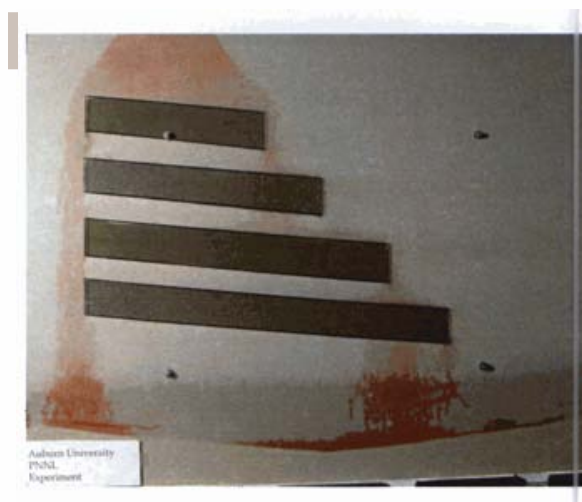

Figure 4-26. Near the final DNAPL distribution, the full collapse of the capillary fringe was observable, which was attribution to a reduction of the surface tension of water caused by DNAPL diffusion in the liquid phase. 


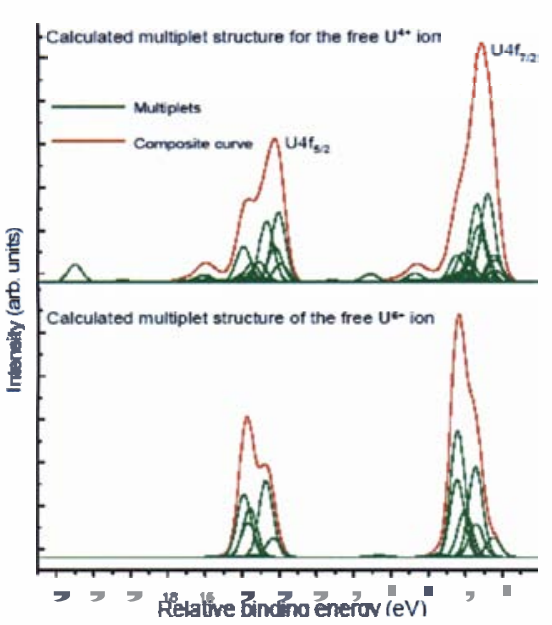

Figure 4-27. Theoretical uranium $4 \mathrm{f} x$-ray photon spectroscopy for the free $\mathrm{U}^{4+}$ ion (top graph), and for the free $\mathrm{U}^{5+}$ ion (lower graph).

\section{A Theoretical and Experimental Investigation of X-ray Photo Electron Spectra for $\mathrm{Cr}$ and $\mathrm{U}$}

ES Ilton, (a) and PS Bagus ${ }^{(b)}$

(a) Pacific Northwest National Laboratory, Richland, Washington

(b) University of North Texas, Denton, Texas

We have developed relativistic $a b$ initio models for the $2 p$ and $3 p$ (Bagus et al. 2004a) spectra of chromium ions in embedded $\mathrm{CrO}_{6}$ clusters of varying symmetry. The models for chromium $2 \mathrm{p}$ predict the relative energies of the multiplets, but more work is required before relative intensities can be calculated. In related work, we discovered a new atomic, manybody effect that significantly improves comparison of theory for manganese $3 \mathrm{~s}$ with experiments (Bagus et al. 2004b). We coined the term 'frustrated auger configuration,' or FAC, to provide an abbreviated description of the effect. We also have begun to investigate the influence of bonding environment on the $4 \mathrm{f}$ spectra of uranium ions. Theoretical models will help to determine the uniqueness of spectral features for uranium (IV), uranium (V), and uranium (VI) that help identify uranium oxidation states. For example, theoretical multiplet and satellite intensities and energies for the uranium $4 \mathrm{f}$ lines of the free $\mathbf{U}^{4}+$ and $\mathrm{U}^{5+}$ ions have been calculated (Figure 4-27). Comparison of the calculated spectrum for $\mathbf{U}^{4}+$ with a spectrum for $\mathbf{U O}_{2}$ indicates that intra-atomic effects are first order. However, inter-atomic effects appreciably decrease the multiplet splitting. Further, theory does not predict the $7-\mathrm{eV}$ satellite that is diagnostic of $\mathbf{U}^{4}+\mathrm{in} \mathrm{UO}_{2}$. This finding is consistent with experimental evidence for an inter-atomic origin of this satellite.

Citations

Bagus PS, ES Ilton, and JR Rustad. 2004a. "Ligand Field Effects for the 3p Photoelectron Spectra of $\mathrm{Cr}_{2} \mathrm{O}_{3}$." Physical Review B 69(20):205112.

Bagus PS, R Broer, and ES Ilton. 2004b. "A New Near Degeneracy Effect for Photoemission in Transition Metals." Chemical Physics Letters 394(1-3):150-154. 


\section{Cryogenic Laser Induced U(VI) Fluorescence Studies of a U(VI) Substituted Natural Calcite: Implications to U(VI) Speciation in Contaminated Hanford Sediments}

\author{
Z Wang, ${ }^{(a)}$ JM Zachara, ${ }^{(a)}$ JP McKinley, ${ }^{(a)}$ SC Smith, ${ }^{(a)}$ and SM Heald(b) \\ (a) Pacific Northwest National Laboratory, Richland, Washington \\ (b) Argonne National Laboratory, Argonne, Illinois
}

The interaction of uranium with carbonate minerals, particularly the calcium carbonate polymorphs calcite and aragonite, has an important bearing on the mobility of contaminant uranium in near-surface environments. In the near surface, uranium is prevalently hexavalent, and occurs as the uranyl ion, $\mathrm{UO}_{2}{ }^{2+}$, which forms strong carbonato complexes. The association of uranyl with carbonate minerals, either sorbed on the mineral surface or incorporated in the mineral structure through co-precipitation, could have a controllinginfluence on its mobility. In addition, the relative solubility and stability of carbonate minerals containing uranyl could determine its long-term availabilityfor remobilization, and co-precipitation of uranyl with carbonate minerals occurs with complex variations. In this study, we used time-resolved, laser-induced fluorescence spectroscopy (TRLFS) to examine the crystallographic environment of uranyl in sediments from a uranyl-bearing infiltration pond with uranium concentrations ranging from 360 to $3300 \mathrm{ppm}$ at DOE's Hanford Site in southeastern Washington state. We also contrast these highly disturbed environmental results with results from a previously studied natural, uranyl-bearing calcite (NUC). The NUC specimen (360 mg U/ kg) originally retrieved from a 13,700-year-old speleothem deposit in the Vinschgau Valley of northernmost Italy was provided by Argonne National Laboratory. X-ray diffraction (XRD) analysis indicated that the sample was more than 99.8 percent pure calcite.

\footnotetext{
Composite time-resolved fluorescence images (TRLFISM) at a series of delay times and gate widths were acquired from the NUC, over an area of approximately $7 \mathrm{~mm}$ by stepping the X-Y translation stage. The distribution of fluorescence (Figure 4-28) showed a relatively small dynamic range, indicating that uranium was distributed more or less uniformly with the sample. Fluorescence was also relatively uniform across measurements at different delay times (Figure 4-28), indicating that individual contributors of the fluorescence signal were uniformly distributed. Time-resolved fluorescence spectra recorded at multiple delay times between 0 and $4 \mathrm{~ms}$ for bulk crystals showed that at the same delay times, the spectral characteristics were similar for spectra obtained on the bulk (TRLFS) and microscopic (TRLFISM) systems, regardless of the spatial location of the area examined or its relative intensity, which was presumably proportional to the relative concentration of uranium within the crystal. However, the fluorescence
}
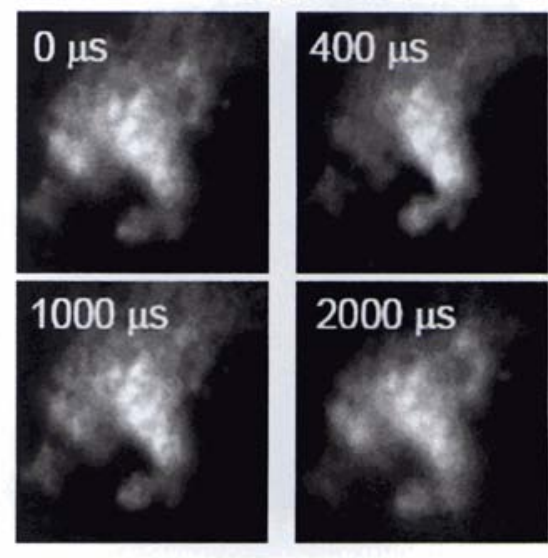

Figure 4-28. TRLFISM of the NUC sample at different delay times. hex $=415 \mathrm{~nm}$. 


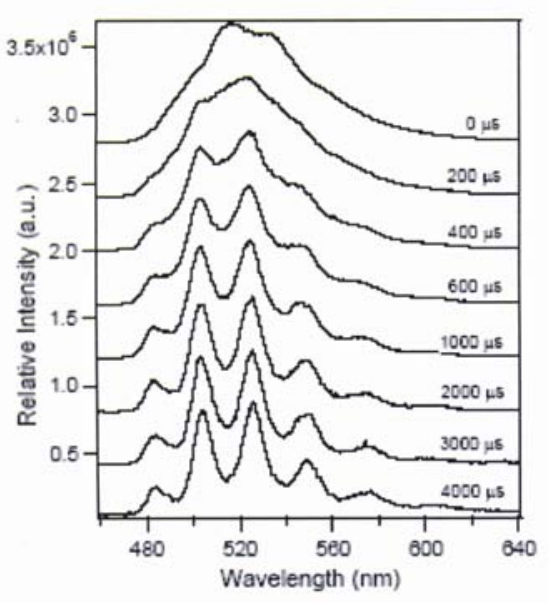

Figure 4-29. Cryogenic TRLFS spectra of a natural Urich calcite at a series of delay times. hex $=415 \mathrm{~nm}$. The maximum intensities were normalized for comparison and the spectra were offset along the Yaxis.

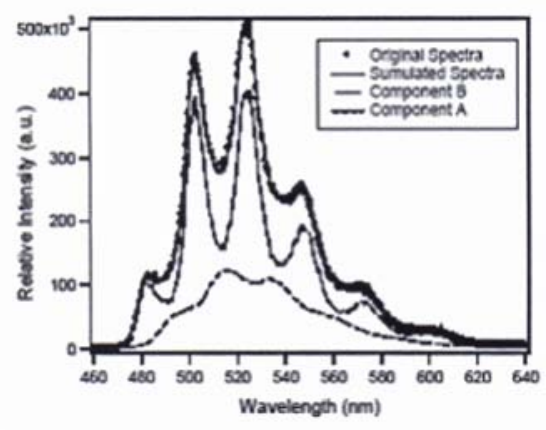

Figure 4-30. Deconvolution of the LHeT time-resolved fluorescence spectra of the uranyl-rich natural calcite at a delay time of $1 \mathrm{~ms}$ into two unique spectral components: $A$ and $B$. spectra showed significant, systematic changes as a runction of the delay time. At shorter delay times, the spectra were less resolved with a spectral maximum located at approximately $513 \mathrm{~nm}$ (Figure 4-29). The spectral resolution increased as the delay time increased, and well-resolved spectra were observed after a delay of about $2 \mathrm{~ms}$.

By assigning the long-delay spectrum to a single component (trace B, Figure 4-30), all of the spectra were definable by linear combinations of two unique spectral components, designated A and B (Figure 4-30). The spectrum of the longer-lived uranyl species (B) in NUC was almost identical to that of uranyl incorporated into aragonite, while the spectrum of the shorter-lived species (A) was similar to that of uranyl incorporated into synthetic calcite. Comparison of our long-lived spectra from the NUC with other results suggested that it was similar to the aqueous tricarbonate species.

Both the sediment samples were examined using TRLFISM and TRLFS with similar procedures applied for the NUC. TRLFISM at a series of delay times indicated that long-lived fluorescence was present. The distribution of fluorescence and the relative fluorescence intensity varied spatially. For some large clasts, fluorescence varied significantly within the clast, and, for a given set of fluorescent clasts, their relative fluorescence intensities varied as a function of delay. 'These observations were clear indications of the presence of multiple uranyl species and of compositional heterogeneity. 'The evolution of TRLFS spectra of sediment NP4-1 (Figure 4-31) displayed a similar pattern to that of the NUC (Figure 4-29), and, in fact, the spectral features of the NP4-1 at delay times longer than $\sim 600 \mu$ s were nearly identical to that of the NUC. The TRLFS spectra of NP4-1 sediment were analyzed following the same procedures for the spectral simulation of the NUC. All of the spectra of NP4-1 could be simulated by the linear combination of two spectral components, one with lower spectral resolution and a spectral maximum at $-517 \mathrm{~nm}$, and another with high spectral resolution and a maximum at $501 \mathrm{~nm}$. The fluorescence decay curves for sediment NP4-1 could also be well fitted by the biexponential function-resulting fluorescence lifetimes of $422 \mu$ s and 92 ps, respectively.

Comparisons of the fluorescence spectra and lifetimes of the uranyl species in NP4-1 with those of the NUC suggested that the same type of uranyl species were present in both samples. The spectral features of the component B in both samples were almost identical, and those for component A were also very close, despite a small shift of the spectral origin towards longer wavelength. Considering the similar fluorescence lifetimes for uranyl species B in the NP4-1 and the NUC, the difference in delay times at which species B became dominant ( $600 \mu$ s for NP4-1 and 2 ms for the NUC) indicated that the relative concentration of this 'aragonite' component was much higher in NP4-1 than in the NUC.

Carbonate minerals are among the most common secondary minerals formed in nature, and dissolved carbonate is a common component of waste solutions containing uranium. The presence of similar uranyl species in the NUC and the Hanford Site sediments implied that the reaction of uranyl with calcite, either through surface precipitation or co-precipitation, facilitated uranium (VI) sorption in the sediments. 


\section{Effects of Sediment Iron Mineralogy on Microbially Mediated Changes in Divalent Metal Speciation: Importance of Ferrihydrite}

DC Cooper, ${ }^{(a)}$ AL Neal, (b) RK Kukkadapu, (c) DC Brewe, ${ }^{(d)}$ A Coby, ${ }^{(e)}$ and FW Picardal (e)

(a) Idaho National Engineering and Environmental Laboratory, Idaho Falls, Idaho

(b) University of Georgia Department of Microbiology, Aiken, South Carolina

(c) W.R. Wiley Environmental Molecular Sciences Laboratory, Richland, Washington

(d) Argonne National Laboratory, Argonne, Illinois

(e) Indiana University, Bloomington, Indiana

Dissimilatory metal-reducing bacteria can influence geochemical processes that subsequently affect the speciation and mobility of metallic contaminants within natural environments. Most investigations into the effect of these bacteria on sediment mineralogy use various synthetic $\mathrm{Fe}$ (III) oxides as the source, providing for well-controlled experiments. However, these oxides do not necessarily emulate the complex mineralogical composition of natural systems, nor do they account for the effect of complex sediment mineralogy on microbial activity and/or microbially induced geochemical processes. Our experiments with a pure dissimilatory metal-reducing bacteria culture (Shewanella putrefaciens $200)$ and a divalent metal $[\mathrm{Zn}(\mathrm{II})]$ indicate that, while complexity in sediment iron mineralogy may not strongly impact the degree of "microbial Fe(III) reducibility," this complexity does alter the geochemical effect of such microbial activity. These experiments also demonstrate that, regardless of sediment composition, the ferrihydrite [poorly crystalline Fe(III)-oxide] content is of central importance.

In this research, changes in $\mathrm{Zn}$ (II) chemistry were quantified through a combination of chemical extractions and x-ray absorption spectroscopy analysis on reduced sediments and sterile controls. In a synthetic goethite and ferrihydrite mixture, $\mathrm{Zn}$ (II) was originally adsorbed to iron oxide surfaces via outer-sphere surface complexes. Upon reduction, the amount of acid-soluble $\mathrm{Zn}$ (II) reduced dramatically and $\mathrm{Zn}$ k-edge extended $\mathrm{x}$-ray absorption finestructure spectroscopy data demonstrated a slight increase in the degree of second order, $\mathrm{Zn}-\mathrm{O}-\mathrm{Zn} / \mathrm{Fe}$ interactions. This shift was not observed in natural sediments containing predominantly quartz, hematite, and kaolinite/illite clays, even though the original $\mathrm{Zn}$ (II) binding was similar to that in the synthetic goethite/ferrihydrite mixture. Here, microbial Fe(III) reduction resulted in the

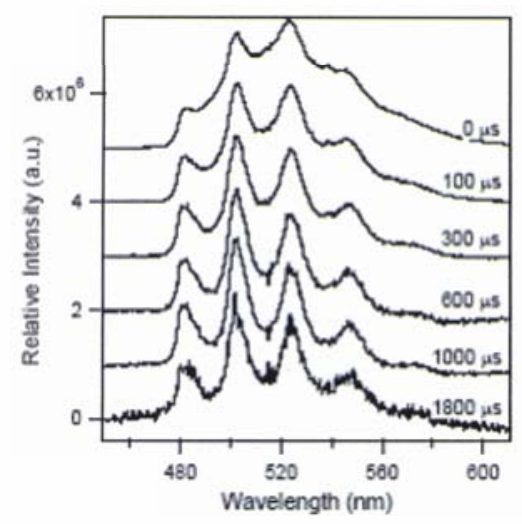

Figure 4-31. Cryogenic TRLFS spectra of NP4-1 sediments at a series of delay times. hex $=415 \mathrm{~nm}$. Gate width $=100 \mu$ s. The maximum intensities of the spectra were normalized and offset along the $\mathrm{Y}$-axis for comparison. 


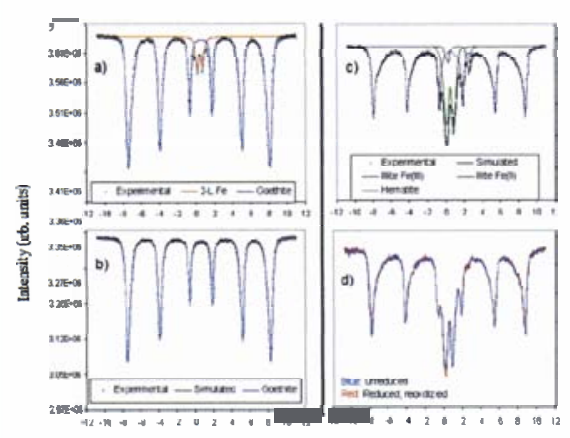

Figure 4-32. Liquid nitrogen (77 K) Mossbauer data for synthetic goethitelferrihydrite. (a) Unreduced synthetic goethite/ ferrihydrite mixture. (b) Reduced and re-oxidized goethite/ ferrihyrite mixture. (c) Unreduced natural sediment. (d) Reduced and re-oxidized sediment. formation of an outer-sphere $\mathrm{ZnCl}_{2}$ complex and did not affect the degree of $\mathrm{Zn}$ (II) acid solubility.

Mossbauer analyses were central to these experiments, and provided key evidence that the differences in the geochemical effect of microbial $\mathrm{Fe}$ (III) reduction were related to the ferrihydrite content of the iron-oxide/sediment. Liquid nitrogen $(77 \mathrm{~K})$ Mossbauer data for synthetic goethite/ferrihydrite reveal a loss of the central doublet upon microbial reduction and subsequent reoxidation, indicating preferential loss of ferrihydrite (Figures 4-32a and b). 'This Mossbauer data (taken at $77 \mathrm{~K}$ ) for the natural sediment (Figures 4-32c and d) reveal a different response to microbial $\mathrm{Fe}$ (III) reduction. Here, a combination of Mossbauer spectroscopy and chemical extractions specific for poorly crystalline iron oxide minerals has indicated that the central doublet (Figure 4-32c) arises from sedimentary clay minerals and not from the ferrihydrite. 'The doublet is representative of structural $\mathrm{Fe}(\mathrm{II})$ in the illite clays, and the sextet arises from the contribution of iron-oxide minerals identified by $\mathrm{x}$-ray diffraction to be hematite. In Figure 4-32d, spectra of the unreduced material and the sample that has been reduced and then re-oxidized are compared. Comparison of these data reveals that, unlike the synthetic goethite/ferrihydrite, microbial $\mathrm{Fe}$ (III) reduction did not permanently alter the iron speciation in this sediment. Also, because previous work has demonstrated that microbial reduction of structural $\mathrm{Fe}$ (III) in illite clay significantly alters the $\mathrm{Fe}$ (II)/ $\mathrm{Fe}$ (III) ratio of the reduced and re-oxidized clay as compared to the unreduced clay, it can be concluded that $\mathrm{Fe}$ (III) in iron oxides was microbially reduced. Roomtemperature Mossbauer data indicated that 1) the percent of total peak area attributable to iron oxide agreed with results from chemical extractions, and 2) apparent Mossbauer parameters did not reflect the presence of siderite or green rust (data not shown).

The preferential loss of ferrihydrite in the synthetic goethite/ferrihydrite stands in contrast to the retention of more crystalline iron oxides (goethite, hematite) in both sediments, and indicates that reactions involving ferrihydrite are responsible for the changes in $\mathrm{Zn}$ (II) acid solubility observed for the synthetic goethite/ferrihydrite. When this evidence is considered in light of earlier studies indicating that the addition of $\mathrm{Fe}$ (II) to ferrihydrite can catalyze transformation of ferrihydrite to goethite, it becomes apparent that ferrihydrite may stimulate a sequence of geochemical reactions that would not occur in its absence. Experiments to specifically test the effect of ferrihydrite addition to iron-bearing sediments are currently underway. 


\section{Self-consistent, Self-interaction Corrected DFT: The Method and Applications to Extended and Confined Systems}

K Tsemekhman, ${ }^{(a)}$ EC Brown, ${ }^{(a)}$ H Jonsson, ${ }^{(a)}$ and EJ Bylaska ${ }^{(b)}$

(a) University of Washington, Seattle, Washington

(b) W.R. Wiley Environmental Molecular Sciences Laboratory, Richland, Washington

We have developed a framework for the self-consistent calculation of selfinteraction corrections (SICs) to the density functional theory (DFT)

(Tsemekhman et al. 2003a, 2003b). 'The technique implements the original method due to Perdew and Zunger and combines two procedures: construction of maximally localized Wannier functions (MLWF, procedure due to Marzari and Vanderbilt and to Silvestrelli) and direct minimization of the DFT+SIC total energy functional. In this formulation, the technique is applicable to both confined and extended systems. While construction of the Wannier functions is a useful tool in the case of molecules and clusters, it is a necessary step for extended systems since self-interaction energies constructed on Bloch functions vanish. Construction of Wannier functions thus provides both a good initial guess and a set of functions for which calculation of nonvanishing SICs is possible. Two direct minimization schemes have been used to solve the nontrivial generalized eigenvalue problem. One of the methods that is similar to the Car-Parrinello method uses the gradient proposed by Goedecker and Umrigar (1998). The other method uses a conjugate gradient algorithm with orthogonality constraints that is based upon the work of Edelman et al. (1998).

'The DFT+SIC method has been applied to several systems for which standard DFT methods do not work well (Figure 4-33). One of the more persistent failures of standard DFT methods has been their failure to yield accurate reaction barriers. However, pragmatic approaches in which the exchange correlation functionals are augmented with small amounts of exact exchange have shown great promise (i.e., B3LYP, PBEO, BH\&HLYP, and mPWH\&HPW91) in improving the accuracy of reaction barriers. Our studies of various chemical reactions showed that SICs can be used in much the same way as exact exchange.

Studies of various chemical reactions showed that including a fractional amount of SICs (40 percent) into a DFT calculation improved the accuracy of calculated reaction energies and barriers considerably.

Another notable failure of standard DFT methods has been their inability to reproduce band gaps. For wide-gap systems (insulators and molecules with large highest occupied molecular orbital-lowest unoccupied molecular orbital gaps), SICs appear to work well. DFT+SIC calculations for $\mathrm{SiO}_{2}, \mathrm{Al}_{2} \mathrm{O}_{3}$, and $\mathrm{TiO}_{2}$
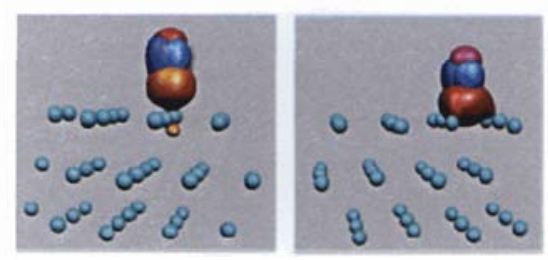

Figure 4-33. DFT-SIC calculations of the chernisortion of carbon monoxide on platinum (111). Left: Top coordination site carbon monoxide orbitals. Right: Face centered cubic coordination site carbon monoxide orbitals. 

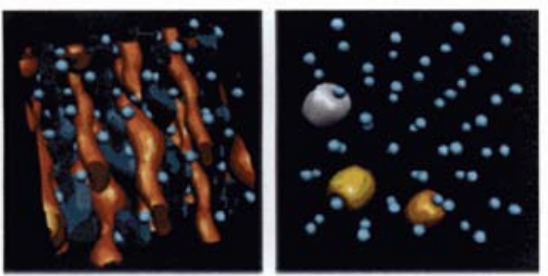

Figure 4-34. Left: Traditional DFT calculations of germanium predict it to be a metal. The Wannier orbitals are not localized, yet a delocalized metallic state persists. Right: DFT+SIC calculations of germanium predict it to be a semiconductor. The Wannier orbitals are localized and are very similar to silicon. crystals, as well as for the $\mathrm{CO}$ molecule, have shown that $0.4^{*} \mathrm{SIC}$ correction predicts values for the gaps as well as for the singlet-triplet splitting in reasonable agreement with the observed values. However, for systems with narrower gaps, the results are more problematic, and the $0.4^{*} \mathrm{SIC}$ correction appears to overcorrect the DFT results. DFT+0.4*SIC calculations for a silicon crystal predicted a minimal gap of $2.25 \mathrm{eV}$ compared to the experimental gap of $1.2 \mathrm{eV}$; in germanium, a $1.2-\mathrm{eV}$ gap is predicted compared to the experimental gap of $0.8 \mathrm{eV}$ (Figure 4-34).

\section{Citations}

Edelman K, TA Arias, and ST Smith. 1998. "The Geometry of Algorithms with Orthogonality Constraints. SIAM Journal on Matrix Analysis and Applications 20(2):303-353.

Goedecker S, and CJ Umrigar. 1998. "Natural Orbital Functional for the ManyElectron Problem." Physical Review Letters 81(4)866-869.

Tsemekhman K, EJ Bylaska, and H Jonsson. 2003a. "Self-Consistent,Self-Interaction Corrected DFT: The Method and Applications to Extended and Confined Systems." Workshop on Ab Initio Electrons Excitations "Theory: Towards Systems of Biological Interest, September 21-24,2003, Donostia International Physics Center, San Sebastian/ Donostia, Spain.

Tsemekhman K, EJ Bylaska, EC Brown, and H Jonsson. 2003b. "Self-Consistent, Self- Interaction Corrected DFT: The Method and Applications to Extended and Confined Systems." Poster presented at the 10th edition of the InternationalCongress on the Applications of Density Functional Theory in Chemistry and Physics, September 7-12,2003, Vrije Universiteit Brussel, Brussels, Belgium.

\section{Effects of Impermeable-Zone Diffusion on Continuous and Intermittent Pump-and- Treat Remediation at Dover Air Force Base, Delaware: Validation of Back Diffusion}

C Liu, (a) and WP Ball(b)

(a) Pacific Northwest National Laboratory, Richland, Washington

(b) Johns Hopkins University, Baltimore, Maryland

Contamination in subsurface sorbing impermeable zones can be viewed either as "sequestered $\mathrm{n}^{\mathrm{n}}$ or "a continuing source," depending on initial conditions, physiochemical properties of the impermeable region, extent of remediation, and conditions of subsequent water withdrawal. The impermeable zones are typically contaminated through diffusive processes from adjacent contaminated permeable zones. In the current study, we used diffusion model simulations and fieldscale measurements to validate that the contaminants in an impermeable zone 
can slowly diffuse back to its adjacent permeable aquifer region as a long-term continuing source during and after aquifer remediation.

\section{Field Research Site, Measurement, and Modeling Approach}

The field research site is located at Dover Air Force Base, Delaware. The subsurface at the site consists of an unconfined aquifer (about $14.4 \mathrm{~m}$ deep) of poorly sorted, fine-to-coarse-grained sand that is underlain by an impermeable aquitard. The aquitard comprises two geologic strata: upper low-sorbing orange silty clay loam and underlying high-sorbing dark gray silt loam (Figure 4-35). 'Ihe aquitard has been contaminated from above as the result of longterm contact with perchloroethene and trichloroethene that are dissolved in the groundwater of the overlying aquifer (Mackay et al. 2000). The primary source of contamination is believed to be approximately 450 meters north (upgradient) of the experimental site and is thought to have first occurred roughly 25 to 35 years before site setup in 1994. Site development included the installation of bentonite-sealed steel sheetpiles to hydraulically isolate portions of the aquifer and aquitard from surrounding groundwater (Mackay et al. 2000). Two experimental cells were created, each of which has plan dimensions of roughly $9.9 \mathrm{~m} \times 3.7 \mathrm{~m}$ and about $15.9 \mathrm{~m}$ depth, with sheetpile penetrating the entire depth of the unconfined aquifer and extending about 1.5 to $2.0 \mathrm{~m}$ into the aquitard. "The concentrations in the aquifer during its remediation were monitored by a multilevel sampling technique and concentrations in the aquitard were monitored by sediment corings before, during, and after "cleanup" of the aquifer region.

Back diffusion modeling involved two steps: inverse estimation of contamination history at the aquifer/aquitard interface within the research site, and diffusion prediction based on the estimated contamination history and known aquifer conditions during and after the period of controlled field remediation. The first step involved Tikhonov-type inverse function estimation to calculate temporal contamination history from measured contaminant profiles in the aquitard at start of field remediation experiments. The mathematical approach and results of the first step were reported elsewhere (Liu and Ball 1999). The current study focused on the prediction of temporal evolution of contaminants in the aquifer and aquitard. "The detailed results were published in Liu and Ball 2002.

\section{Summary of Modeling Results and Comparison with Measurements}

The diffusion-predicted concentrations matched well with the measured values (Figure 4-35). The predictions were made using two estimated contamination history functions at the aquifer/aquitard interface based on two vertical contaminant profiles in the aquitard (at core locations called PPC 11 and PPC 13) measured at the start of aquifer remediation. Predictions were made after 7.5 months at core location PPC 16 and 15 months at PPC 19 after the cleanup of the aquifer site. Predicted and measured upward concentration
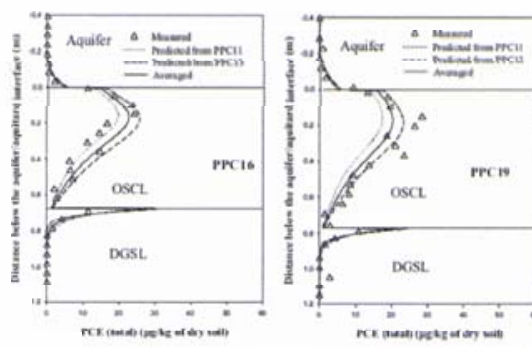

Figure 4-35. Measured and predicted vertical perchloroetheneconcentration profiles at PPC 16 and 19. 
gradients near the aquifer/aquitard interface, downward concentration gradients in the deep aquitard, and the reappearance of perchloroethene in the aquifer region after aquifer remediation are direct evidences of back diffusion. The total (aqueous and sorbed) concentration discontinuity at the orange silty clay loam and dark gray silt loam and orange silty clay loam/aquifer interfaces is due to the sorption discontinuity. 'The close match between diffusion predicted and measured profiles indicated that diffusion was a dominant transport process within this impermeable aquitard.

\section{Citations}

Liu C, and W P Ball. 2002. "Back Diffusion of Chlorinated Solvent Contaminants from a Natural Aquitard to a Remediated Aquifer under Well-Controlled Field Conditions: Predictions and Measurements." Ground Water 40(1):175-184.

Liu C, and W P Ball. 1999. "Application of Inverse Methods to Contaminant Source Identification from Aquitard Diffusion Profiles at Dover AFB, DE." Water Resources Research 35(7):1975-1985.

Mackay DM, RD Wilson, MP Brown, W P Ball, G Xia, and DP Durfee. 2000. "A Controlled Field Evaluation of Continuous Versus Pulsed Pump-and-Treat Remediation of a VOC-Contaminated Aquifer: Site Characterization, Experimental Setup, and Overview of Results." Journal of Contaminant Hydrology 41(1-2):81-131.

\section{Surface Structure Effects on Direct Reduction of Iron Oxides by Shewanella oneidensis}

AL Neal, ${ }^{(a)}$ KM Rosso, ${ }^{(b)}$ G G Geesey, ${ }^{(c)}$ and YA Gorby, ${ }^{(b)}$ and BJ Little ${ }^{(d)}$

(a) University of Georgia, Athens, Georgia

(b) Pacific Northwest National Laboratory, Richland, Washington

(c) Montana State University, Bozeman, Montana

(d) U.S. Naval Research Laboratory, Stennis Space Center, Mississippi

Dissimilatory iron-reducing bacteria (such as Shewanella spp., Geobacter spp. and Fenibacterium spp.) are afforded clear environmental significance in the cycling of iron due to reduction of $\mathrm{Fe}$ (III) associated with iron oxides/oxyhydroxides and clays, the reactivity of the resulting $\mathrm{Fe}$ (II), and potential effects on the mobility of other metals and radionuclides. Solution chemistry effects upon biogenic Fe3+-mineral reduction and subsequent precipitate formation have been intensively studied and, at least in laboratory reactors, are increasingly well-understood aspects of the bioreduction process. However, the atomic and electronic structures of mineral surfaces are rarely considered to be relevant to dissimilatory bacterial reduction of iron and manganese minerals. In this regard, surface area and thermodynamics are more commonly considered. Here, 
we took a first step toward understanding the nature of the influence of mineral surface structure upon the rate of electron transfer from Shewanella oneidensis strain MR-1 outer membrane proteins to the mineral surface and the subsequent effect on cell activity.

In this study (Neal et al. 2003), we addressed this issue by employing a series of three single-crystalline iron-oxide surfaces for comparison of bioreduction rates, namely hematite (001), magnetite (111), and magnetite (100). 'The structures of the three surfaces (Figure 4-36) are expected to differ significantly for many reasons, not the least of which is that while hematite possesses the corundum structure and has univalent iron $\left(\alpha-\mathrm{Fe}_{2} \mathrm{O}_{3}\right)$, magnetite possesses the inverse spinel structure and has mixed-valentiron $\left[\left(\mathrm{Fe}^{2+}{ }_{2}, \mathrm{Fe}^{3+}\right) \mathrm{O}_{4}\right]$. We used a combined experimental and theoretical approach. Anaerobic cell growth on the three well-characterized single-crystalline iron-oxide surfaces in flow reactors was monitored and was used as a proxy for bioreduction activity. A combination of $a b$ initio modeling and Marcus theory was used to predict electron transfer rates from a model outer-membrane cytochrome molecule to the three different oxide surfaces, and to evaluate the principal structural and energetic factors that lead to intrinsic differences in the surfaces as electron acceptors for bioreduction.

A sequential increase in maximum cell density supported by the three mineral faces was observed with magnetite (100) sustaining the lowest, magnetite (111) an intermediate, and hematite (001) a significantly greater density. This sequence was not reflected in the number of cells collected in the effluent where, relatively, the two magnetite surfaces were associated with the greatest effluent cell density. Thus, despite having similar surface areas, differences between cell accumulation at the surface and cells released into solution are observed between the three crystal faces. The mode of growth of S. oneidensis on hematite appears to be surface related; on magnetite proportionately more daughter cells leave the surface. However, total cell productivity on the magnetite (100) surface is much reduced compared to the other two faces, consistent with this face supporting the lowest surface-associated population.

$A b$ initio molecular modeling was applied to estimate the rate of electron transfer from an idealized utermembrane cytochrome (OmcA) to $\mathrm{Fe}^{3+}$ sites associated with the three oxide surfaces. The model predicts rapid electron transfer kinetics overall, with a strong dependence on both the surface structure and on the distance of separation between a heme center in utermembrane cytochrome and the oxide surface. Several lines of evidence suggest heme/surface distances pertinent to the current system fall in the range 5 to $9 \AA$. Within this expected range of separation, electron transfer to hematite (001) turned out to be faster than for magnetite surfaces at distances less than about $6 \AA$, while the reverse was found true for longer distances. Thus, if the actual electron transfer distances are predominantly at the small end of the range, the electron transfer model is consistent with the experimental cell accumulation results, and the importance of interfacial electron transfer in controlling the activity of attached cells remains a valid possibility. If actual distances are greater, then our findings indicate interfacial electron transfer is not a valid control. Since actual electron transfer distances are not easily determined, we cannot draw definitive conclusions regarding the role of interfacial electron transfer as a control on cell a)

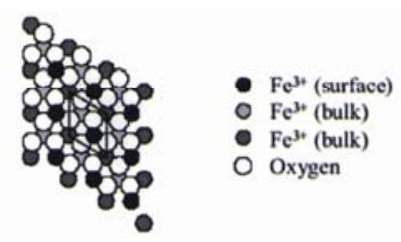

b)

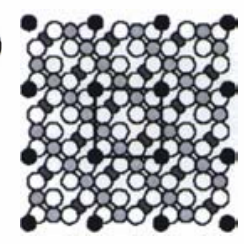

- $\mathrm{Fe}^{3+} \mathrm{Tet}_{\text {(surface) }}$ - $\mathrm{Fe}^{3+2+}{ }_{\mathrm{OAt}}^{\mathrm{Tet}}$ (surface) $\mathrm{Fe}^{3+}{ }_{\text {Tat }}$ (bulk) O Oxygen

c)

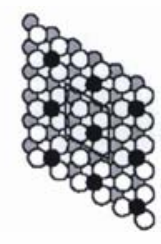

- $\mathrm{Fe}^{3+}$ Tet (surface)

Oxygen

Figure 4-36. Ball models of the surface structures of a) hematite (001), b) magnetite (100), and c) magnetite (111) as viewed down the surface normal direction. Only the upper-most oxygen plane is shown along with iron atoms coordinated to it. The tetrahedral and octahedral iron sublattices in magnetite are differentiated by subscripts. The octahedral iron sublattice in magnetite consists of equal numbers of $2+$ and $3+$ valence states. 


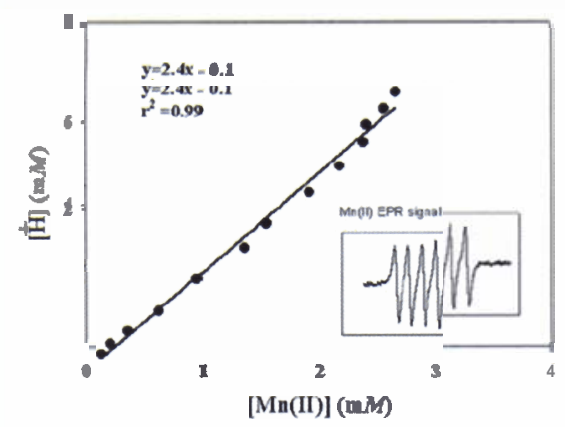

Figure 4-37. Stoichiometry of the reductive dissolution of manganite by catechol. The insert confirmed that manganite was reduced to the $\mathrm{Mn}(\mathrm{II})$ product based on the diagnostic IR six-line spectrum.

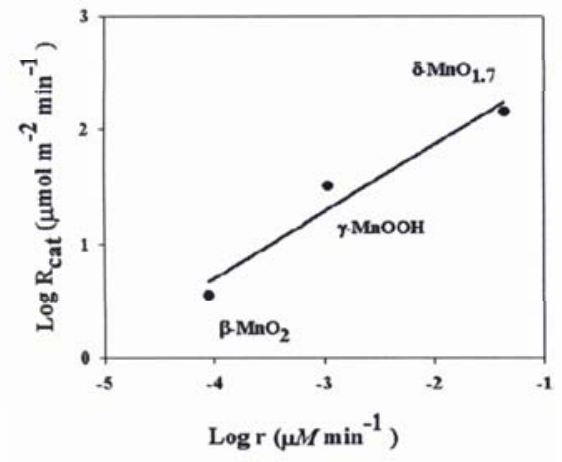

Figure 4-38. Relationship between the initial rate of $\mathrm{Mn}(\mathrm{III})$ extraction by pyrophosphate, defined as available $\mathrm{Mn}(\mathrm{III})$, and the reductive dissolution rate bv catechol. activity at this time. Clearly, however, the model findings are consistent with the experimental observations that surface structure is an important factor for this interfacial system.

Citation

Neal AL, KM Rosso, GG Geesey, YA Gorby, and BJ Little. 2003. "Surface Structure Effects on Direct Reduction of Iron Oxides by Shewanella oneidensis." Geochimica et Cosmochimica Acta 67(23):4489-4503.

\section{Role of Mn(III) Oxidation of Soil Organic Matter}

CJ Matocha, ${ }^{(a)}$ DL Sparks, ${ }^{(b)}$ RK Kukkadapu, ${ }^{(c)}$ and JE Amonette ${ }^{(d)}$

(a) University of Kentucky, Lexington, Kentucky

(b) University of Delaware, Newark, Delaware

(c) William R. Wiley Environmental Molecular Sciences Laboratory. Richland, Washington

(d) Pacific Northwest National Laboratory, Richland, Washington

$\mathrm{Mn}(\mathrm{II})$ and $\mathrm{Mn}(\mathrm{IV})$ have received the most attention in aquatic chemistry (Morgan 1967) until recently, when solid $\mathrm{Mn}$ (III) oxides and soluble $\mathrm{Mn}$ (III) complexes have shown to be environmentally significant oxidants (Stone and Morgan 1984; Luther 1990; Kostka et al. 1995; Klewicki and Morgan 1998; Luther et al. 1998). Mn(III) solid phases would be expected to be more kinetically reactive than $\mathrm{Mn}$ (IV) minerals, based on frontier molecular orbital theory; however, no detailed studies have been conducted. 'This study investigated the reductive dissolution kinetics of naturally occurring solid $\mathrm{Mn}(\mathrm{III}, \mathrm{IV})$ (hydr) oxide minerals by comparing several phases [birnessite $(\delta$ $\left.\mathrm{MnO}_{2}\right)$; manganite $(\gamma-\mathrm{MnOOH})$ and pyrolusite $\left.\left(\beta-\mathrm{MnO}_{2}\right)\right]$ with varying levels of structural $\mathrm{Mn}(\mathrm{III})$ in their reactivity with catechol, a model organic ligand of soil organic matter (Evanko and Dzombak 1998).

Reactivity studies of solid $\mathrm{Mn}(\mathrm{III}, \mathrm{IV})$ (hydr) oxide minerals with catechol were followed in situ using an EPR stopped flow technique (Matocha et al. 2001) and diffuse reflectance spectroscopy. Manganite $(\gamma-\mathrm{MnOOH})$, a pristine $\mathrm{Mn}(\mathrm{III})$ solid phase, was reduced by catechol to the $\mathrm{Mn}$ (II) product (Figure 4-37). It was found that initial reduction dissolution rates by catechol measured with EPR spectroscopy did not scale with total structural Mn(III), but rather with available $\mathrm{Mn}$ (III) based on initial rates of pyrophosphate-extractable $\mathrm{Mn}$ (III) (Figure 4-38). 'Ihe positions of the $\mathrm{Mn}$ (III) ligand field bands derived from diffuse reflectance spectroscopy analyses suggested different coordination environments for structural $\mathrm{Mn}$ (III) in manganite and birnessite, which explained the different levels of availability. 'Therefore, the role of $\mathrm{Mn}$ (III) in different coordination environments merits special attention in abiotic cycling of soil organic matter and manganese. 
Evanko CR, and DA Dzombak. 1998. "Influence of Structural Features of Sorption of NOM-Analogue Organic Acids to Goethite." Environmental Science and Technology 32(19):2846-2855.

KlewickiJK, and JJ Morgan. 1998. "Kinetic Behavior of Mn(III) Complexes of Pyrophosphate, EDTA, and Citrate." Environmental Science and Technology 32(19):2916-2922.

Kostka JE, G W Luther III, and KH Nealson. 1995. "Chemical and Biological Reduction of $\mathrm{Mn}(\mathrm{III})$-Pyrophosphate Complexes: Potential Importance of Dissolved $\mathrm{Mn}(\mathrm{III})$ as an Environmental Oxidant." Geochimica et Cosmochimica Acta 59(5):885-894.

Luther GW, III. 1990. "The Frontier-molecular-orbital Theory Approach in Geochemical Processes." In Aquatic Chemical Kinetics: Reaction Rates of Processes in Natural Water. W. Stumm (ed.) pp. 173-198. Wiley-Interscience, New York.

Luther GW, III, D T Ruppel, and C Burkhard. 1998. "Reactivity of Dissolved Mn(III) Complexes and Mn(IV) Species with Reductants: Mn Redox Chemistry Without a Dissolution Step?" In Mineral-Water Interfacial Reactions: Kinetics and Mechanisms. DL Sparks and TJ Grundl (eds.) pp. 265-280. ACS Symposium Ser. No. 715, Washington, DC.

Matocha CJ, DL Sparks, JE Amonette, and RK Kukkadapu. 2001. "Kinetics and Mechanism of Birnessite Reduction by Catechol." Soil Science Society of AmericaJournal 65(1):58-66.

Morgan JJ. 1967. "Chemical Equilibria and Kinetic Properties of Manganese in Natural Waters." In Principles and Applications of Water Chemistry. SD Faust and JV Hunter (ed.). pp. 561-624. Wiley, New York.

Stone AT, and JJ Morgan. 1984. "Reduction and Dissolution of Manganese(III) and Manganese(IV) Oxides by Organics. 1. Reaction with Hydroquinone." Environmental and Science Technology 18(6):450-456.

\title{
Iron Sulfides and Sulfur Species Produced at (001) Hematite Surfaces in the Presence of Sulfate-Reducing Bacteria
}

\author{
AL Neal, (a) S Techkarnjanaru, ${ }^{\left({ }^{(a)}\right.}$ A Dohnalkova, ${ }^{(b)}$ DE McCready, ${ }^{(c)}$ B M \\ Peyton, ${ }^{(d)}$ and GG Geesey(a) \\ (a) Montana State University, Bozeman, Montana \\ (b) Pacific Northwest National Laboratory, Richland, Washington \\ (c) W.R. Wiley Environmental Molecular Sciences Laboratory, Richland, \\ Washington \\ (d) Washington State University, Pullman, Washington
}

In the presence of sulfate-reducing bacteria (Desulfovibrio desulfuricans), hematite $\left(\alpha-\mathrm{Fe}_{2} \mathrm{O}_{3}\right)$ dissolution is affected and hydrogen sulfide-the product 


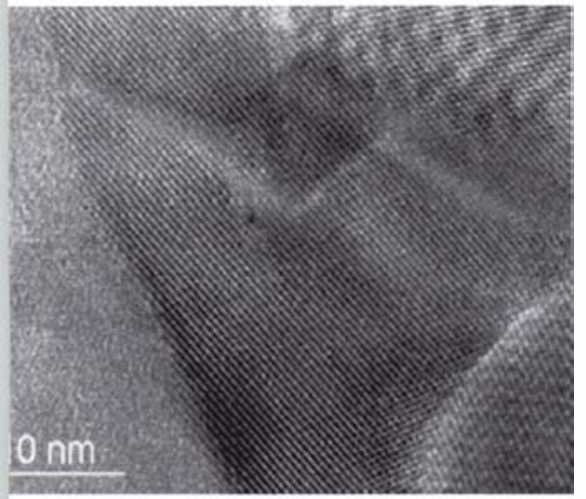

Figure 4-39. HRTEM image of a crystalline layer present on a hematite (001) surface after three months of incubation with D. desulfuricans. D-spacingsare 2.6, 3.8, and $5.1 \mathrm{~A}$. of sulfate reduction-is released. As a consequence, ferrous ions are free to react with excess $\mathrm{H} 2 \mathrm{~S}$ to form insoluble iron sulfides. Iron sulfides produced by sulfate-reducing bacteria have been studied and described as mackinawite and greigite (Rickard 1969; Herbert et al. 1998). In both studies, iron sulfides were produced by free-living bacteria in liquid culture with added ferrous ions. However, most subsurface bacterial activity is likely to be associated with mineral surfaces (Ghiorse and Wilson 1988: Costerton et al. 1995). We have therefore chosen to study and identify iron sulfides produced by the bacteria Desulfovibrio desulfuricans associated with a hematite $\left(\alpha-\mathrm{Fe}_{2} \mathrm{O}_{3}\right)$ surface as the most abundant iron oxide along with goethite found in soils and sediments. We report that the iron sulfide formed at the mineral surface under these conditions is pyrrhotite-like.

Two Desulfovibrio desulfuricans strains, G20 and Essex 6, were grown in lactate medium and incubated with natural specular hematite $\left(\alpha-\mathrm{Fe}_{2} \mathrm{O}_{3}\right)$ for three months. After removing the samples from the culture medium, hematite and the associated precipitates were observed using epifluorescent microscopy, and analyzed by X-ray photoelectron spectroscopy (XPS), XRD, and HRTEM (Figure 4-39). Throughout incubation, culture media were evaluated for the formation of iron sulfides in suspension, concluding that sulfite production was confined to the hematite surfaces. XPS spectra of all hematite surfaces exposed to $D$. desulfuricans compared to the unexposed surfaces showed a presence of a prominent photopeak at $708.4 \mathrm{eV}\left(\mathrm{X}^{2}=1.15\right)$ which was assigned to $\mathrm{Fe}^{2}+-\mathrm{S}$.In the $\mathrm{S} 2 p$ region, the $\mathrm{S}^{2-}$ peak was identified at $161.3 \mathrm{eV}$, the $\mathrm{S}_{2}{ }^{2-}$ peak at $162.5 \mathrm{eV}, \mathrm{S}^{2-}$ at $163.9 \mathrm{eV}$ and $\mathrm{SO}_{4}{ }^{2-}$ at $168 \mathrm{eV}\left(\mathrm{X}^{2}=2.63\right)$. The XPS spectra of the hematite surface exposed to Essex 6 in the presence of sulfate suggested that the sulfide layer was of sufficient thickness to mask any hematiterelated signal. The XRD pattern of a hematite exposed to bacteria for three months showed peaks with d-spacings of $1.28,1.81,2.04$, and $2.09 \AA$, confirming that a crystal phase forms on a surface; however, due to a lack of heterogeneous crystal orientation with so few lines, a reliable assignment of an unequivocal crystal structure is not possible, particularly since the 2.09 - $\AA$ line could be assigned to pyrrhotite as well as mackinawite and greigite.

HRTEM images of the hematite surface precipitates illustrate the presence of numerous crystals; d-spacings of 2.6, 3.8, and 5.1 Å were consistently measured. However, Fourier transformation of the 2.6-A pattern, the most commonly observed, yielded a hexagonal crystal structure substantiating the identification of the iron-sulfide phase as a pyrrhotite.

Iron sulfides have an important role in both the sulfur and iron cycles. 'The intermediate metastable species, mackinawite and greigite, have a key influence upon the iron and sulfur balance. Any reduction in ambient $\left[\mathrm{Fe}^{2+}\right]$ or [ $\left.\mathrm{S}^{2-}\right]$ results in remobilization of the precipitates, as is oxidation. "This remobilization attains greater significance when one considers that other potentially toxic elements are often co-precipitated with iron. The formation of pyrrhotite, a species considered stable at low temperatures, will greatly limit the remobilization of iron, sulfur, and other co-precipitates caused by environmental fluctuations in iron, sulfur, and 02 . Polysulfides, being a reduced species, represent a reactive form of elemental sulfur and together with monosulfides are 
responsible for maintaining trace metal concentrations in anoxic sediments at relatively high concentrations. Sulfide/polysulfide formation by sulfate-reducing bacteria has the potential to greatly affect sulfur, iron, and other trace metal concentrations in anoxic soils and sediments, of significance not only to the sulfur and iron cycles but also to the bioavailability of toxic trace metals.

The geological significance of sulfate-reducing bacteria activity derives from the production of $\mathrm{H}_{2} \mathrm{~S}$, resultant from sulfate reduction, and its subsequent reaction with $\mathrm{Fe}^{2+}$ to form iron sulfides. Such sulfides are common components of both recent and ancient sediments and include the 1) tetragonal, sulfur-deficient $\mathrm{Fe}^{2+}$ sulfide, mackinawite ( $\left.\mathrm{FeS}-\mathrm{Fe}_{1.07} \mathrm{~S}\right) ; 2$ ) the mixed-valence thiospinel, greigite $\left(\mathrm{Fe}_{2}{ }^{3+}, \mathrm{Fe}^{2}+\mathrm{S}_{4}\right)$; and 3$)$ the $\mathrm{Fe}^{2+}$ - polysulfide, pyrite $\left(\mathrm{FeS}_{2}\right)$. Additionally, H2S production by sulfate-reducing bacteria has implications for steel corrosion (Hamilton 1991) and bioremediation of heavy metal pollution in anoxic environments (Webb et al. 1998).

\title{
Citations
}

Costerton JW, Z Lewandowski, DE Caldwell, DR Korber, and HM Lappin-Scott. 1995. "Microbial Biofilms." Annual Review of Microbiology 49:711-745.

Ghiorse WC, and J T Wilson. 1988. "Microbial Ecology of the Terrestrial Subsurface." Advances in Applied Microbiology 33:107-172.

Hamilton, WA. 1991. "Sulphate-ReducingBacteria and Their Role in Microbially Influenced Corrosion." In Microbially Influenced Corrosion and Biodeterioration (ed. $N$. J. Dowling et al.), pp. i-iv. Institute for Applied Microbiology.

Herbert RB, Jr., SG Benner, AR Pratt, and DW Blowes. 1998. "Surface Chemistry and Morphology of Poorly Crystalline Iron Sulfides Precipitated in Media Containing Sulfate-Reducing Bacteria." Chemical Geology 144(1-2):87-97.

Rickard DT. 1969. "The MicrobiologicalFormation of Iron Sulphides." Stockholm Contributions in Geology 20:49-66.

Webb JS, S McGinness, and HM Lappin-Scott. 1998. "Metal Removal by SulfateReducing bacteria from Natural and Constructed Wetlands." Journal of Applied Microbiology 84(2):240-248.

\section{Mossbauer Spectroscopic Study of Iron Redox in Silicate Glasses}

\author{
RK Kukkadapu, ${ }^{(a)}$ GL Smith, ${ }^{(b)} \mathrm{H} \mathrm{Li,}$, (b) JV Crum, ${ }^{\text {(b) }}$ MC Weinberg, ${ }^{\text {(c) }}$ and \\ H Poisl ${ }^{(c)}$
}

(a) W.R. Wiley Environmental Molecular Sciences Laboratory, Richland, Washington

(b) Pacific Northwest National Laboratory, Richland, Washington

(c) University of Arizona, Tucson, Arizona 
High-level waste glasses contain significant quantities of iron. Vitrification processing conditions and waste glass composition can affect the ironoxidation state in glass, which in turn can influence the tendency of liquidliquid immiscibility, or phase separation, of the final glass waste formed. An understanding of the effects of the iron redox ratio on liquid-liquid immiscibility is important for immobilization of high-level waste because glass phase separation can adversely impact chemical durability of the final waste form (Tomozawa 1979; Taylor 1990). The objective of this study is to determine the nature of the iron species and the iron-oxidation state in glasses employing colorimetery and complementary spectroscopic techniques, such as absorption spectroscopy in the ultraviolet-visible and infrared wavelength ranges, and ${ }^{57} \mathrm{Fe}-\mathrm{Mössbauer}$. The results will be correlated with glass-phase separation work currently being conducted at the University of Arizona.

${ }^{57} \mathrm{Fe}-\mathrm{Mössbauer}$ spectroscopy is an iron-specific technique with greater sensitivity than XRD; iron-oxidation states and the local environment are identifiable by Mossbauer for samples with iron concentrations as little as $0.5 \mathrm{wt} \%$. The ${ }^{57} \mathrm{Fe}-$ Mossbauer technique provides information on the valence and coordination state, crystal field strengths [e.g., low-spin and high-spin $\mathrm{Fe}(\mathrm{II})]$, magnetic ordering temperatures, etc. In contrast to XRD, it also provides information on compounds that do not exhibit long-range order (poorly crystalline or amorphous materials). Common iron-oxide phases, such as magnetite and hematite, are readily distinguished from each other and from ferrous compounds.

Transmission ${ }^{57} \mathrm{Fe}$-Mössbauer spectra were collected at room temperature (about $25^{\circ} \mathrm{C}$ ) and liquid helium temperature $(4.2 \mathrm{~K})$ using a $50-\mathrm{mCi}(1.85-\mathrm{MBq})$ ${ }^{57} \mathrm{Co} / \mathrm{Rh}$ single-line thin source. The velocity transducer (Wissel, Germany) was operated in the constant-acceleration mode $(23 \mathrm{~Hz}, \pm 10 \mathrm{~mm} / \mathrm{sec})$. Data were acquired on 1024 channels and then folded to 512 channels to give a flat background and a zero velocity position corresponding to the center shift (CS or $\mathrm{O}$ ) of a metallic-iron foil at room temperature. Calibration spectra were obtained with a $20-\mu \mathrm{m}$-thick (a-Fe foil (Amersham, England) placed in exactly the same position as the samples to minimize any error due to changes in geometry. The transmitted radiation was recorded with an argon-krypton proportional counter. Approximately $180 \mathrm{mg}$ of powder sample (particle size $<75 \mu \mathrm{m}$ ) was mixed uniformly with petroleum jelly in a 0.5 -inch-thick and 0.5 -inch-diameter copper holder sealed at one end with clear tape. 'The amount of sample chosen corresponded to the ideal absorber thickness that provided the largest signal-to-noise ratio in the given collection time. For the $4.2 \mathrm{~K}$ analyses, an oxygen impermeable film, which would be stable at $4.2 \mathrm{~K}$ (Arlon, Inc., California) was used instead of tape. Petroleum jelly was used to fill the sample space volume, and the end was sealed with tape or Arlon film. The $4.2 \mathrm{~K}$ Mossbauer measurements were performed using a top-loading Janis exchangegas cryostat. The temperature was continuously monitored with a Nichrome thermocouple and was within $\pm 0.5 \mathrm{~K}$. A Lakeshore Model DRC-93CA temperature controller was used to control the temperature. 'The measured isomer shifts were quoted relative to the Fe foil at room temperature. The entire drive-source assembly was external to the cryostat. In other words, only the absorber (sample) was cooled to $4.2 \mathrm{~K}$. 
The unfolded spectra obtained were folded and evaluated with the MOS MOD and Recoil programs (University of Ottawa, Canada) using the Voigt-based hyperfine parameter distribution method (Rancourt and Ping 1999). The Voigt-based method assumes a certain number (V) of generalized sites (quadrupole splitting distributions and/or hyperfine field distributions), each having their own continuous distribution (quadrupole splitting distributions or hyperfine field distributions). Each normalized site-specific quadrupole splitting distribution or hyperfine field distribution is composed of a certain number of Gaussian components $(\mathrm{C})$ being the sum of more than one Voigt line. The center shift $\left({ }^{\mathrm{TM}}\right)$ of each quadrupole splitting distribution site is related to its quadrupole splitting, (A or QS, as $\delta=\delta_{0}+\delta_{1} \Delta$ where 60 is the value of 6 when the distributed hyperfine parameter is zero, and 61 is the coupling of 6 to the distributed hyperfine parameter. For the hyperfine field distribution site, the 6 is related to its Zeeman splitting, z, as $6=\delta_{0}+\delta_{1} z$; and its quadrupole shift parameter, $\Sigma$, site is related to its $z$, as $\Sigma=\sum_{0}+\sum 1 z$, where $\Sigma_{0}$ is the value of $\mathbf{z}$ when $\mathbf{z}$ (which is distribution) is zero $\operatorname{and} \Sigma_{1}$ is the coupling of $\sum$ to the distributed hyperfine parameter.

Liquid-liquid immiscibility in sodium silicates has been extensively studied (Hammel 1965; Porai-Koshits and Averjanov 1968). From the data available in the literature, a composition of $18.56 \mathrm{~mol} \% \mathrm{Na}_{2} \mathrm{O}$ and $81.44 \mathrm{~mol} \% \mathrm{SiO}_{2}$ is expected to be outside the immiscibility dome at and above glass transition temperature. Therefore, the effect of an iron-oxidation state on this baseline composition can be investigated. 'The effects of iron concentration and oxidation state on the glass phase separation is part of the overall research objective. In this study, the silicate glass doped with $0.5 \mathrm{~mol} \% \mathrm{Fe}_{2} \mathrm{O}_{3}$ (UA-3) was prepared at $1600^{\circ} \mathrm{C}$ at ambient conditions. Figure 4-40 shows Mossbauer spectra collected at room temperature and $4.2 \mathrm{~K}$. The room-temperature spectrum shows an asymmetric doublet (in the region -2 to $3 \mathrm{~mm} / \mathrm{sec}$ ) with a non-linear background. The outer peak ( 2.2 to $2.5 \mathrm{~mm} / \mathrm{sec}$ ) of the doublet is characteristic of high-field components of paramagnetic $\mathrm{Fe}$ (II) (Ono and Ito 1964), while the inner peak is a mix ture of low-field components of $\mathrm{Fe}(\mathrm{II})$ and both the doublets of Fe(III) (Greenwood and Gibb 1971). A shoulder to this effect is evident at the high-energy (higher velocity) end of the peak. The nonlinear background, which is uncommon in this type of glass, indicates the presence of magnetic iron species or clusters that are amorphous in nature. "The $\mathrm{Fe}$ (II)/iron-total ratio of the sample can be derived by fitting the spectrum, provided the valence of iron contributing to the nonlinear background is known. Cooling down the sample to lower temperatures, usually to those of liquid nitrogen or liquid helium, would resolve this ambiguity. At low temperatures (usually below $30 \mathrm{~K}$ for iron minerals), the spin-spin and spinlattice relaxation processes slow down, such that their $t_{1 / 2}$ become longer than that of the ${ }^{57} \mathrm{Fe}$ excited state, and magnetic spectra can be observed (Hawthorne 1988). For paramagnetic iron materials, the magnetic field normally changes due to these relaxation effects, with the changes being fast compared to the lifetime of the 57Fe excited state.

Experimental and simulated spectra of the sample at $4.2 \mathrm{~K}$ are shown in Figure 4-40. The experimental spectrum exhibited apart from the

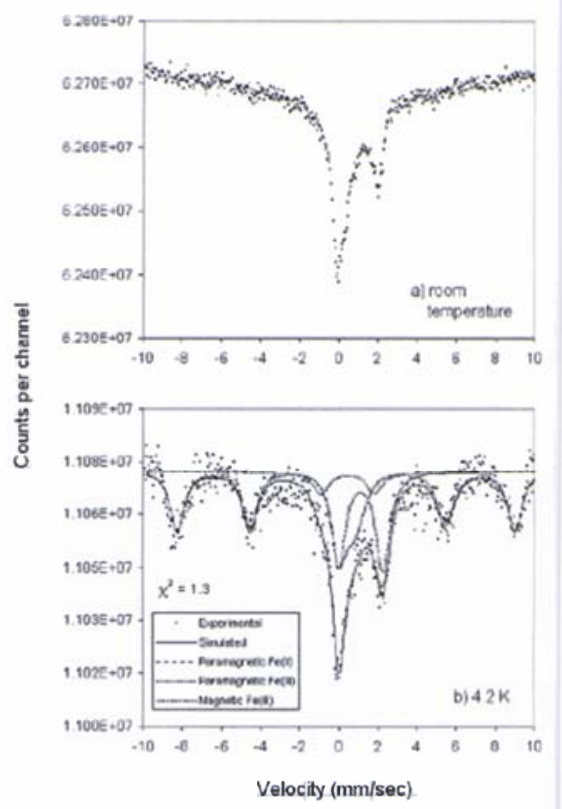

Figure 4-40. Room-temperature and 4.2K Mossbauer spectra. 
asymmetric doublet due to paramagnetic iron sites are a sextet characteristic of magnetic iron. Simulation of the pattern yielded the following parameters for the sextet: $6=0.42 \mathrm{~mm} / \mathrm{sec} ; \mathrm{A}=-0.05 \mathrm{~mm} / \mathrm{s}$; magnetic hyperfine field $=$ 53.5 T. 'The derived 6 and A parameters are characteristic of Fe(III); Fe(II) sites exhibit larger A values. The hyperfine field value of $53.5 \mathrm{~T}$ and other Mossbauer parameters are similar to hematite $\left(\alpha-\mathrm{Fe}_{2} \mathrm{O}_{3}\right)$; for example, a weakly ferromagnetic hematite exhibits a hyperfine field of 53.3 T at 4.2K, (Murad et al. 1988) whereas magnetite $\left(\mathrm{Fe}_{3} \mathrm{O}_{4}\right)$ and other commonly occurring iron oxides exhibit lower hyperfine fields. We invoked a hyperfine distribution model to fit the spectrum: the derived full-width at half maximum was $0.40 \mathrm{~mm} / \mathrm{sec}$ versus $0.20 \mathrm{~mm} / \mathrm{sec}$ (natural line width) expected for an elemental sextet, suggesting that a distribution of local chemical environments exists in the sample.

Additional studies will be performed to investigate the origin of the magnetic behavior of the glass with such low iron concentration.

The asymmetric doublet at $4.2 \mathrm{~K}$ is similar to the one at room temperature. Fit parameters indicated that paramagnetic $\mathrm{Fe}$ (II) and $\mathrm{Fe}$ (III) contributed to this signal. The derived parameters for $\mathrm{Fe}(\mathrm{II})(6=1.13 \mathrm{~mm} / \mathrm{sec} ; \mathrm{A}=2.27 \mathrm{~mm} / \mathrm{s})$ and $\mathrm{Fe}(\mathrm{III})(6=0.76 \mathrm{~mm} / \mathrm{sec} ; \mathbf{A}=0.37 \mathrm{~mm} / \mathrm{s})$ are similar to those reported in the literature. A comparison of room temperature and $4.2 \mathrm{~K}$ spectra shows that the ratio of the areas of the high-energy peak to the low-energy peak is similar in both, suggesting that the nonlinear background present at room temperature is solely due to magnetic Fe(III).

The $\mathrm{Fe}$ (II)/iron-total ratio was calculated from the relative areas of the different iron sites of the $4.2 \mathrm{~K}$ spectrum. In the literature, the iron redox ratio in glass is conventionally determined by the ratio of the area under the spectrum for $\mathrm{Fe}$ (II) over the total area of the spectrum, (Levy et al. 1976; Mysen et al. 1980) assuming that the ratio off $\mathrm{Fe}(\mathrm{II}) / f \mathrm{Fe}(\mathrm{III})$ is not substantially different from unity (where $f$ is the fraction of these atoms that is recoil-free). 'The spectral area percentages derived from the $4.2 \mathrm{~K}$ spectrum due to different iron components are as follows: magnetic $\mathrm{Fe}(\mathrm{III})$ is 46.4 percent, paramagnetic $\mathrm{Fe}$ (III) is 23.4 percent, and paramagnetic $\mathrm{Fe}(\mathrm{II})$ is 30.2 percent. Based on these values, the ratio of $\mathrm{Fe}$ (II)/iron-total was calculated. "The ratio obtained (0.30) was slightly higher than 0.22 determined by using a calorimetric method. In the calorimetric method, $\mathrm{Fe}$ (II) could be oxidized during the dissolution of glass in acid at ambient temperature, resulting in a slightly lower measured $\mathrm{Fe}(\mathrm{II})$ concentration.

Magnetic behaviors of low and high iron (1 to 4 mol\% $\mathrm{Fe}_{2} \mathrm{O}_{3}$ ) glasses with a different iron redox ratio will be further studied using Mossbauer in a collaboration with the glass-phase separation study. Because of the use of a powder sample with large surface area for the measurement, it is important to accurately determine the surface (top $100 \mathrm{~nm}$ or so) iron-oxidation states, especially for the glasses partially reduced; a conversion electron Mossbauer spectroscopy setup, currently being developed at EMSL, would be employed for a surface study. In addition, synchrotron Mossbauer spectroscopy studies are planned to gather information on f-factors and relaxation phenomena. The use of transmission electron microscopy to characterize the glass microstructures at a nanoscale is also planned, which could provide further 
understanding of the origin of the magnetic behaviors in glass with iron partially reduced.

\section{Citations}

Greenwood NN, and T C Gibb. 1971. Mossbauer Spectroscopy. Chapman and Hall Ltd., London.

Hammel JJ. 1965. "Experimental Evidence for Spinodal Decomposition in Glasses of the $\mathrm{Na} 20 \mathrm{SiO}_{2}$ System." 7th International Congress on Glass.

Hawthorne FC. 1988. "Mossbauer-Spectroscopy."Reviews in Mineralogy 18:255-340.

Levy RA, CHP Lupis, and PA Flinn. 1976. "Mossbauer Analysis of Valence and Coordination of Iron Cations in $\mathrm{SiO} 2-\mathrm{Na} 2 \mathrm{O}-\mathrm{CaO}$ Glasses" Physics and Chemistry of Glasses 17(4): 94-103.

Murad E. 1988. "Properties and Behavior of Fe Oxides as Determined by Mossbauer Spectroscopy." In: Iron in Soils and Clay Minerals. Eds. J W Stucki, BA Goodman, and U Schwertmann, pp. 309-350 Reidel Publishing Company: Dordrecht, Holland.

Mysen BO, F Seifert, and D Virgo. 1980. "Structure and Redox Equilibria of IronBearing Silicate Melts." American Mineralogist 65(9-10):867-884.

Ono K., and A Ito. 1964."Mossbauer Study of Magnetic Properties in Ferrous Compounds." Journal of the Physical Society of Japan 19(6):899-907.

Porai-Koshits EA, and VI Averjanov. 1968. Journal of Non-CrystallineSolids, 1(1): 29-38 (1968).

Rancourt DG, and JY Ping. 1999. Nuclear Instruments \& Methods in Physics Research Section B-Beam Interactions With Materials and Atoms B58:85.

Taylor P. 1990. Review of Phase Separation in Borosilicate Glasses with Reference to Nuclear Fuel Waste Immobilization. AECL-10173. Atomic Energy of Canada Limited, Whiteshell Laboratories, Pinawa, Manitoba, Canada.

Tomozawa M. 1979. "Phase Separation in Glass." In Treatise on Materials Science and Technology Vol. 17. Eds. M Tomozawa and R H Doremus, pp. 71-113. 


\section{Recent Upgrades}

\section{0-MHz Cold Probe}

In early 2004, the High-Field Magnetic Resonance Facility enhanced its available NMR capabilities with the arrival of an $\mathrm{H}(\mathrm{C} / \mathrm{N})$ pulsed-field gradient triple resonance cold probe for the 600/51 Inova NMR spectrometry system.

'Ihe system has contributed to the collection of high-quality data for a number of peer-reviewed scientific projects. "The cold probe system allows EMSL users to reduce system thermal noise and increase signal-to-noise three- to four-fold. Greatly improved signal-to-noise allows the user to either reduce data collection times, resulting in an increase in the number of experiments that can be performed during a given time, or to reduce sample concentrations so that more challenging sample systems can be examined. The improvement in research productivity afforded by the cold probe, as well as the ability to examine less tractable systems by NMR, has increased the facility's ability to support leadingedge scientific research.

\section{0-MHz INOVA Console and Pentaprobe}

'Ihe 600-MHz INOVA console replaced an outdated 14-year-old console that could not be operated using the current generation of software. "The new 600-MHz console can run BioPack, a state-of-the art suite of biological experiments that simplifies the collection of protein structure data. BioPack offers a range of experiments that could not be performed using the old console. The new console features a deuterium-decoupling channel, which opens up a new range of samples and experimental approaches to collecting protein structure data on large proteins. As part of the console upgrade, a $\mathrm{H}(\mathrm{NCPD})$ probe was purchased that will increase signal-to-noise on existing experiments, as well as add capability for additional structural experiments of RNA and DNA. 'This probe is being installed in early 2005.

\section{Solid-State NMR Probe Development}

During the past year, researchers from the High-Field Magnetic Resonance Facility and PNNL developed several unique solid-state probes for use at EMSL. Two new cryogenic probes were constructed for the 9.4-tesla and 11.7-tesla NMR magnets. The 9.4-tesla probe is triple-tuned to zinc, nitrogen, and hydrogen, and the 11.7-tesla probe is an HX probe capable of tuning from ${ }^{25} \mathrm{Mg}$ to ${ }^{31} \mathrm{P}$. The techniques used to develop the new capacitor and vacuum feed required by these probes have enabled all of the tuning elements to be located in the cryogenic environment. 'Thus, the tuning ranges are no longer limited and the probes can be tuned to a wide range of nuclei. In addition to these two new probes, a new slow-spinning 5-mm cross-polarization,magic-angle spinning (CPMAS) probe was constructed for use in the Varian and Bruker imaging gradients sets. 'This probe has enabled a new class of experiments to be implemented for studying metabolic changes in cellular systems. Finally, a high-speed 3.2-mm CPMAS HX probe with a tuning range of ${ }^{87} \mathrm{Sr}$ to ${ }^{23} \mathrm{Na}$ was the first CPMAS probe developed for the 21.1-tesla magnet, equipped on the facility's state-of-the-art 900-MHz NMR spectrometer. Because of its high spinning speed $(>23 \mathrm{kHz})$ and high-performance radio frequency section, this

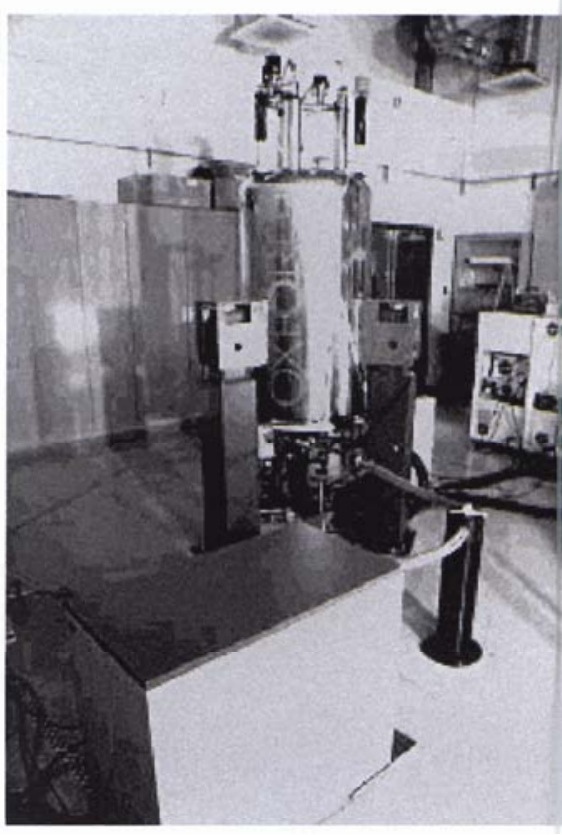

$600-\mathrm{MHz}$ cold probe

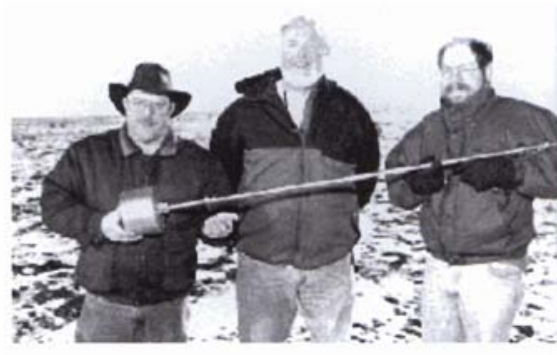

A cryogenic solid-state NMR probe and its developers. 
probe will enable several new solid-state experiments to be performed at this high field. One of the first user projects to benefit from the new probe was a study of environmentally challenging waste site cleanup samples containing strontium.

\section{EPR Console and Pulsed Bridge}

The console and microwave bridge on the Pulsed EPR/ENDOR/ELDOR spectrometer were upgraded in November 2004 to an EleXsys E580 console and a SuperX-FT bridge. This upgrade provides EMSL researchers with a modern operating system and user interface for the spectrometer and enables remote operation of the spectrometer over the Internet. A great improvement in resolution and throughput are afforded by the pulse programmer and signal averager in the new console, and with many samples, a ten-fold increase in signal-to-noise ratio is achievable. The microwave bridge offers improved sensitivity and pulse shaping ability, and is capable of producing microwave pulses as narrow as 2 ns. Two independent microwave sources in the bridge permit routine pulsed ELDOR measurements of nanoscale distances and facilitate a number of hyperfine selective ENDOR measurements. The SuperX-FT bridge includes a strip-line pulse former unit with four microwave channels and a pair of microwave pulse former units that provide four additional microwave channels in addition to the ELDOR channel. Pulse sequences are being ported over to the new system, and calibrated data collection for research projects is currently underway. The new console is supported by a new Xepr data analysis software suite and the Xsophe simulation suite, with enhanced, extensible processing and simulation of experimental data.

\section{Future Direction}

As the High-Field Magnetic Resonance Facility prepares to meet the requirements of two new EMSL Scientific Grand Challenge projects and strategically fit facility resources to match the capability needs of some of the future CATs, careful efforts will be made to select resource enhancements, update equipment, and develop novel capabilities. This effort will require additional capabilities that will obviously impact data collection for a large number of projects, Scientific Grand Challenges, CATs, and open-call proposals. These additional capabilities could include a cold probe for a high-field instrument or investment in more specific capabilities, such as a unique probe design for in-situ catalysis experiments as part of the EMSL Catalysis CAT. The needs for the upcoming Scientific Grand Challenges will emerge further in 2005 as scientific milestones are set.

Recent upgrades to the pulsed EPR spectrometer will expand the range of experiments for EMSL's Biogeochemistry Grand Challenge. Designing a new "flat-coil ${ }^{\mathrm{n}}$ probe for use with biosolid-state experiments could help with the study of protein-membrane orientation, both in cyanobacterium and other systems identified by EMSL's user base. This type of probe, paired with EMSL's 900M Hz NMR spectrometer, could yield a powerful combination for gaining new understanding of membrane biology. Another unique capability, the study of NMR-active radionuclides, is being developed on 300-MHz NMR system. This capability promises to advance the understanding of fundamental radionuclide chemistry, with applications to Hanford Site cleanup. 
In fall 2005, the High-Field Magnetic Resonance Facility plans to receive an $800-\mathrm{MHz}$ cold probe system that was purchased in mid-2004. This system will be equipped with the highest signal-to-noise capability of all of EMSL's biological liquids NMR probes (a 50 percent increase over the $600-\mathrm{MHz}$ cold probe system received in 2004). 'This will be a welcome improvement for analysis of difficult samples such as larger macromolecules and other samples less tractable at millimolar concentrations. New sample systems that were previously intractable for study with the earlier technology will be practical at tens of micromolar concentrations.

Other capability development projects that may be pursued in 2005 include a 4-mm H X CPMAS (VT-capable; ${ }^{87} \mathrm{Sr}-{ }^{23} \mathrm{Na}$ ) for EMSL's state-of-the-art 900 MHz NMR spectrometer; this system could assist with a variety of solid-state material projects and would provide needed sensitivity to help with studies such as those related to strontium environmental cleanup.

Researching and taking advantage of opportunities to support distinguished science capabilities, as well as maintaining state-of-the-art systems, will keep productivity of the High-Field Magnetic Resonance Facility high and its capabilities relevant.

\section{Facility Staff}

\section{David Hoyt \\ Technical Lead \\ (509) 373-9825 david.hoyt@pnl.gov}

Araceli Perez

Administrative Secretary

(509) 376-2548

Ariceli.Perez@pnl.gov

Sarah D. Burton

Senior Research Scientist

(509) 376-1264

sarah.burton@pnl.gov

Joseph J. Ford

Senior Research Scientist

(509) 376-2446

joseph.ford@pnl.gov

\author{
Michael J. Froehlke \\ Technician \\ (509) 376-2391 \\ michael.froehlke@pnl.gov \\ Nancy G. Isern \\ Research Scientist \\ (509) 376-1616 \\ nancy.isern@pnl.gov \\ Donald N. Rommereim \\ Senior Research Scientist \\ (509) 376-2671 \\ don.rommereim@pnl.gov
}

Jesse A. Sears, Jr.

Technician

(509) 376-7808

jesse.sears@pnl.gov

\section{Key Staff}

Key staff, besides those listed above, include Michael K Bowman, Garry W Buchko, Herman M Cho, John R Cort, Paul D Ellis, Jian Zhi Hu, Michael A Kennedy, David W Koppenaal, Andrew S Lipton, Paul D Majors, Kevin R Minard, 'Theresa A Ramelot, Robert A Wind, John R Bagu, and Kate McAteer. 


\title{
Protein NMR Structures from the Northeast Structural Genomics Consortium in 2004
}

\author{
JR Cort, ${ }^{(a)}$ T A Ramelot, ${ }^{(a)}$ JM Aramini, ${ }^{(b)}$ GJ Kornhaber, ${ }^{(b)}$ DA Snyder, ${ }^{(b)}$ \\ MC Baran, ${ }^{(b)}$ GVT Swapna, ${ }^{(b)}$ A A Yee, ${ }^{(c)}$ B Wu, ${ }^{(c)}$ G T Montelione, ${ }^{(b)}$ \\ $\mathrm{CH}$ Arrowsmith, ${ }^{(\mathrm{c})}$ and MA Kennedy, ${ }^{(\mathrm{a})}$ \\ (a) Pacific Northwest National Laboratory, Richland, Washington \\ (b) Center for Advanced Biotechnology and Medicine, Rutgers University, \\ Piscataway, New Jersey \\ (c) University of Toronto, Toronto, Ontario, Canada
}

Proteins comprise the machinery of the cell-they convert energy from one form to another, assemble and repair DNA, transmit signals from outside the cell to the nucleus, and serve numerous other roles. For many proteins, roles have yet to be discovered. The large fraction of proteins that are functionally uncharacterized presents a major challenge for today's efforts to reach a holistic understanding of the cell, which typically contains thousands of different proteins. Knowledge of the structure of a particular protein of unknown function can aid the development and testing of hypotheses about its function. The collected knowledge of the structures of a representative sampling of all the proteins in the cell will enhance our ability to describe the cell as a networked system of interacting parts, many of which are proteins.

The Northeast Structural Genomics Consortium (NESG) is a group of investigators at several institutions in the United States and Canada, funded by the Protein Structure Initiative of the National Institutes of Health and engaged in a project using both NMR spectroscopy and X-ray crystallography for highthroughput protein structure determination. EMSL is one of several facilities at which NMR data is collected for the NESG. This data is distributed to groups at Rutgers University, University of Toronto, the State University of New York at Buffalo, and the Biological Sciences Division of PNNL. Since the NESG collaboration was established in 1999, 175 total structures have been deposited in the Protein Data Bank, of which about half were determined using NMR methodologies. Of these, 28 structures were determined from complete datasets collected at PNNL; partial datasets collected at PNNL have been used to assist in elucidating more than 25 additional structures. Data collected at PNNL has resulted in 26 papers published in the scientific literature, four of which were selected as journal covers. In 2004, complete data sets for nine proteins, and partial data sets (usually select experiments at high field) for nine additional proteins were recorded for the NESG. Full data sets typically represent four to five weeks of instrument time; the partial data sets take between one and three weeks of time. Altogether, approximately 60 weeks of EMSL instrument time were devoted to structural genomics in 2004. Ten refined structures were 
deposited to the Protein Data Bank at www.rcsb.org. The structure that is deposited consists of atomic coordinates in cartesian space, though in Figure 4-41 they are shown as ribbon cartoon representations. 'They represent the final product produced from the data collected at EMSL and are available in the Protein Data Bank for use by scientists everywhere.

Following structure determination, NESG scientists compare the structures to others in the structural data base. Often two proteins with dissimilar amino acid sequences that adopt similar structures will have functional similarities. Other clues to protein function can be derived from identification of particular arrangements of amino acid side chains in the structure. Such analyses often suggest further experimental studies and can lead to new discoveries in biochemistry and molecular biology.

Figure 4-41.

Structures of 10 proteins, eight from bacteria and two from humans. Detailsare available at www.nesg. org. The structures were deposited in the Protein Data Bank (www.rcsb.org) in 2004. The structures were determined with data collected at the EMSL High-Field Magnetic Resonance Facility for the NESG. For each protein structure, the NESG and Protein Data Bank identification numbers are given.
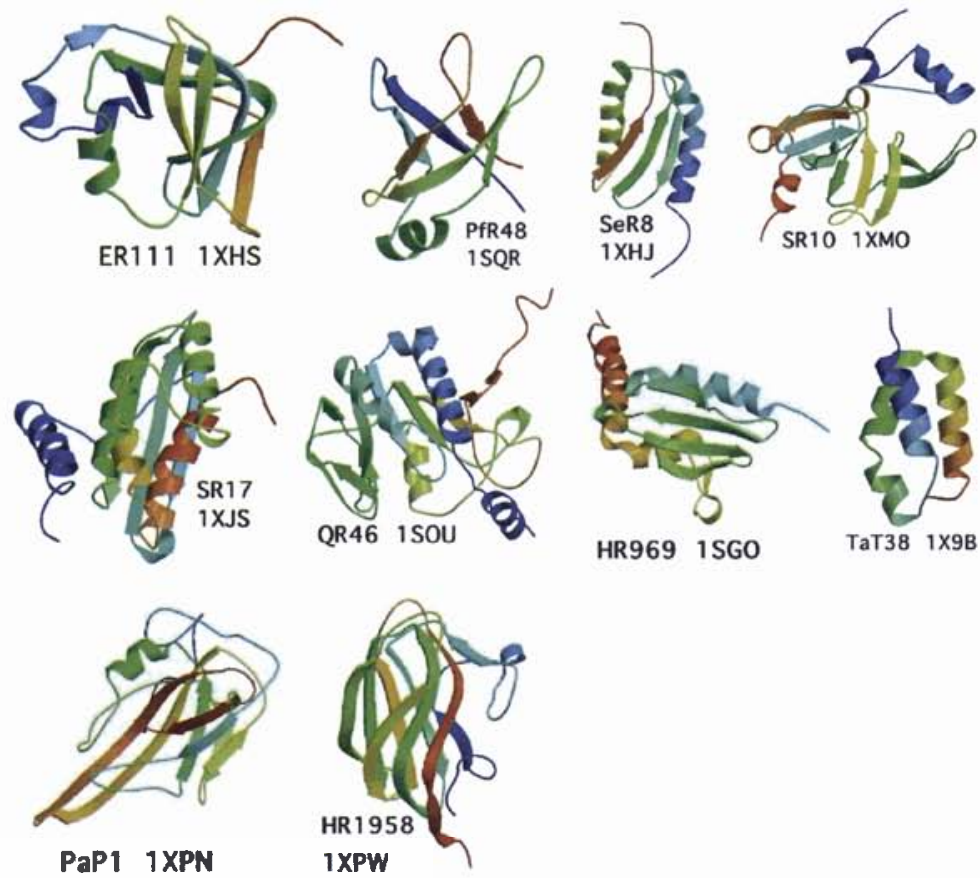

HR969 1SGO 


\section{The following publications weregenerated as part of this work:}

Cort JR, Y Chiang, D Zheng, M A Kennedy, and G Montelione. 2002. “NMR Structure of Conserved Eukaryotic Protein ZK652.3 from C. elegans: a Ubiquitin-like Fold." Proteins. Structure, Function, and Genetics 48(4):733-736.

Cort JR, SS Mariappan, CY Kim, MS Park, TS Peat, GS Waldo, T C Terwilliger, and M A Kennedy. 2001. "Solution Structure of Pyrobaculum aerophilum DsrC, an Archaeal Homologue of the Gamma Subunit of Dissimilatory Sulfite Reductase." European Journal of Biochemistry/FEBS 268(22):5842-50.

Cort JR, A Yee, A M Edwards, C H Arrowsmith, and M A Kennedy. 2000. "Structure-Based Functional Classification of Hypothetical Protein MTH538 from Methanobacterium thermoautotrophicum." Journal of Molecular Biology 302(1):189-203.

Cort JR, EV Koonin, PA Bash, and M A Kennedy. 1999. "A Phylogenetic Approach to Target Selection for Structural Genomics: Solution Structure of YciH." Nucleic Acids Research 27(20):4018-4027.

Kennedy MA, G Montelione, CH Arrowsmith, and JL Markley. 2002. "A Role for NMR in Structural Genomics." Journal of Structural and Functional Genomics 2(3):155169.

Powers R, T B Acton, Y Chiang, R Paranji,JR Cort, M A Kennedy, J Liu, L Ma, B Rost, and G T Montelione. 2004. ${ }^{11} \mathbf{H},{ }^{13} \mathrm{C}$ and ${ }^{15} \mathrm{~N}$ Assignments for the Archaeglobus fulgidis Protein AF2095." Journal of Biomolecular N M R 30: 107-108.

Ramelot TA, JR Cort, S Goldsmith-Fischman, GJ Kornhaber, R Xiao, R Shastry, T B Acton, B Honig, G T Montelione, and M A Kennedy. 2004. "Solution NMR Structure of the Iron-sulfur Cluster Assembly Protein U (IscU) with Zinc Bound at the Active Site." Journal of Molecular Biology 344: 567-583.

Ramelot TA, JR Cort, A Yee, F Liu, MB Goshe, A M Edwards, R D Smith, CH Arrowsmith, T E Dever, and M A Kennedy. 2002. "Myxoma Virus Immunomodulatory Protein M156R is a Structural Mimic of Eukaryotic Translation Initiation Factor eIF2a." Journal of Molecular Biology 322:943-954.

Savchenko A, N Krogan, JR Cort, E Evdokimova,J M Lew, A A Yee, L SánchezPulido, M A Andrade, A Bochkarev, M A Kennedy, J Greenblatt, T Hughes,

C H Arrowsmith, J Rommens, and A M Edwards. "The Shwachman-Bodian-Diamond Syndrome Protein Family is Involved in RNA Metabolism." 2005. Journal of Biological Chemistry (in press).

Yee A, X Chang, A Pineda-Lucena, B Wu, A Semsesi, B Le, T A Ramelot, G M Lee, S Bhattacharyya, P Gutierrez, A Denisov, C Lee, JR Cort, G Kozlov, J Liao, G Finak, L Chen, D Wishart, W Lee, LP Mclntosh, K Gehring, M A Kennedy, A M Edwards, and CH Arrowsmith. 2002. "An NMR Approach to Structural Proteomics." Proceedings of the National Academy of Sciences of the United States of America 99(4):1825-1830. 


\title{
Variable-Temperature MAS NMR Spectroscopic Study of Incorporated and Sorbed ${ }^{133} \mathrm{Cs}$ and ${ }^{23} \mathrm{Na}$ in Zeolites
}

\author{
Y Deng, ${ }^{(a)}$ J Ford, ${ }^{(b)}$ SD Burton, ${ }^{(b)}$ JB Harsh, ${ }^{(a)}$ and M Flury ${ }^{(a)}$ \\ (a) Washington State University, Pullman, Washington \\ (b) W.R. Wiley Environmental Molecular Sciences Laboratory, Richland, \\ Washington
}

Mineral alteration has been observed in sediments underlying high-level radioactive waste storage tanks that have leaked at DOE's Hanford Site in Richland, Washington. The alteration may change sediment properties for incorporation, adsorption, and desorption of pollutants from the waste leaks. Cancrinite, sodalite, Linde Type A zeolite, and allophane have been observed in our simulation experiment. The new mineral phases, especially the colloid-sized particles, may facilitate the transport of radioactive nuclides in the vadose zone. Radioactive ${ }^{133} \mathrm{Cs}$ is a major radiation source in the tank waste. Feldspathoids and zeolites contain cages and channels in which $\mathrm{Cs}^{1+}$ can exist as ion pairs with $\mathrm{OH}^{1}-\mathrm{NO}_{3}{ }^{1}, \mathrm{NO}_{2}{ }^{1-}, \mathrm{CO}_{3}{ }^{2-}, \mathrm{Cl}^{1-}$, and $\mathrm{SO}_{4}{ }^{2-}$. Water in the cages of the minerals may also affect the chemical environment of the cations. In this project, we used variable temperature magic-angle-spinning NMR to investigate the chemical environment of cesium and sodium inside feldspathoids and zeolites (Norby et al. 1998).

We carried out this experiment on EMSL's Varian/Chemagnetics Infinity-Plus 400-MHz NMR spectrometer. The temperature varied from $213 \mathrm{~K}$ to $498 \mathrm{~K}$, and the rotors were spun at $8 \mathrm{kHz}$. Cancrinite, sodalite, and Linde Type A zeolite showed different responses to the temperature changes, two examples of which are given in Figure 4-42. For the sodalite in which cesium was incorporated, the ${ }^{23} \mathrm{Na}$ magic-angle spinning spectrum recorded at $293 \mathrm{~K}$ shows a main peak at -5.7 ppm and a shoulder at around 2.4 ppm (Figure 4-42a). Decreasing temperature to $213 \mathrm{~K}$ causes the peaks to merge to one broader peak. Increasing temperature enhanced the intensity of the 2.4-ppm peak and weakened and shifted the peak at $-5.7 \mathrm{ppm}$. The two peaks were best resolved in the temperature range of 353 to 423 $\mathrm{K}$. Increasing temperature above $448 \mathrm{~K}$ caused the collapse of the 2.4-ppm peak. These changes suggest that the chemical environment in sodalite is homogenous for sodium at low temperatures. Motion averaging of cations in sodalite does not seem to be the major contributor to the singularity of the NMR peak at low temperatures. Increasing temperature reduced moisture in the cages and likely caused the different chemical environments. We may assign the -5.7-ppm peak to the hydrated sodium and the 2.4-ppm peak to the dehydrated sodium.

The ${ }^{133} \mathrm{Cs}$ NMR spectra of Linde Type A zeolite (Figure 4-42b) are very different from the spectra of cancrinite or sodalite (data not shown). Linde Type A zeolite has only one narrow peak at different temperatures, thus indicating
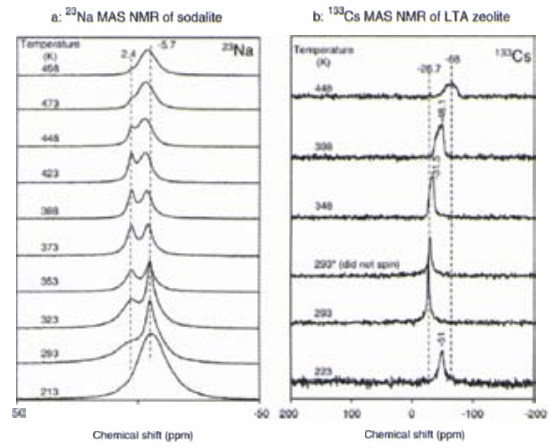

Figure 4-42. Variable temperature magic-angle spinning NMR spectra of samples containing cesium. (a) ${ }^{23} \mathrm{Na}$ of sodalite and $(b){ }^{133} \mathrm{Cs}$ of Linde Type A zeolite. (Sodium is the dominant cation in both samples.) 


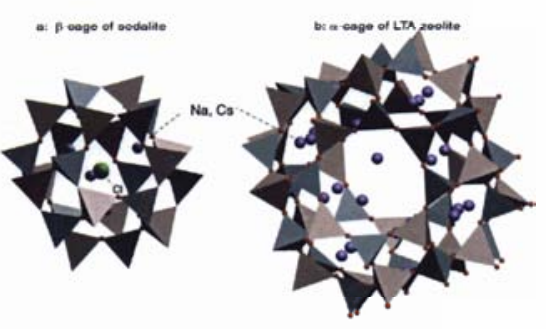

Figure 4-43. Cages in (a) sodalite and (b) Linde Type A zeolite. Linde Type A zeolite contains both $\mathrm{a}$ - and $\boldsymbol{\beta}$-cages, but cations stay in the large a-cage, which has more freedom for the migration of cations. very homogenous chemical environments or high motion of the cesium in Linde Type A zeolite. We recorded a pattern at $293 \mathrm{~K}$ without spinning the rotor [the third spectrum from bottom (Figure 4-42b)], and the peak is nearly as narrow as the one recorded with a spin rate of $8 \mathrm{kHz}$. This narrow peak width suggests that the mobility of cations in Linde Type A zeolite is nearly as high as those in solutions. This is likely the result of the large size of the (-cage in Linde Type A zeolite (Figure 4-43b).

Two manuscripts are ready for submission based on this data, and it is a continuation of earlier work conducted at EMSL using the electron microscope facilities (Zhao 2004).

\section{Citations}

Norby P, FI Poshni, AF Gualtieri,JC Hanson, and CP Grey. 1998. "Cation Migration in Zeolites: An In Situ Powder Diffraction and MAS NMR Study of the Structure of Zeolite Cs(Na)-Y During Dehydration." Journal of Physical Chemistry B 102(5):839-856.

Zhao HT, YJ Deng, JB Harsh, M Flury, and JS Boyle . 2004. "Alteration of Kaolinite to Cancrinite and Sodalite by Simulated Hanford Tank Waste and Its Impact on Cesium Retention." Clays and Clay Minerals 52(1):1-13.

\section{Magic-Angle-Spinning NMR of Highly Radioactive Ceramics: ${ }^{29}$ Si NMR of Zircons Containing $10 \mathrm{wt} \%{ }^{239} \mathrm{Pu}$ and ${ }^{238} \mathrm{Pu}$}

\author{
I Farnan, ${ }^{(a)} \mathrm{H}$ Cho, ${ }^{(b)}$ WJ Weber, ${ }^{(b)}$ R W Scheele, ${ }^{(b)}$ NR Johnson, ${ }^{(a)}$ and \\ A Kozelisky(b) \\ (a) University of Cambridge, Cambridge, United Kingdom \\ (b) Pacific Northwest National Laboratory, Richland, Washington
}

We have developed a protocol for acquiring magic-angle-spinning NMR spectra of highly radioactive samples. To our knowledge, this is the first report of such an experiment. These methods have been used to quantify the radiation damage occurring in manmade ceramic samples containing ${ }^{239} \mathrm{Pu}$ and ${ }^{238} \mathrm{Pu}$. Internal radioactive decay is accelerated in these systems, and they serve as a model for the long-term behavior of potential ceramic radionuclide waste forms.

Naturally occurring zircons $\left(\mathrm{ZrSiO}_{4}\right)$ can contain the radioactive elements uranium and thorium at levels up to several thousand parts per million. 'They exhibit a mixed crystalline and amorphous character because of the large number of radioactive decay events that have taken place within them over geological time. Magic-angle spinning NMR measurements can enumerate silicon atoms in the amorphous and crystalline phases and have provided, for the first time (Farnan and Salje 2001), a quantitative measure of the amount of structural damage that occurs in terms of the number of permanently displaced atoms per $\boldsymbol{\alpha}$-decay. These 
"active" experiments test the feasibility of the rigorous quantification of radiation damage/resistance in ceramics that are candidates for radionuclide immobilization.

'The key safety consideration in an experiment with a radioactive sample is to prevent contamination or harmful exposures of the operator or equipment. 'This is accomplished by preparing the sample in a nondispersibleform. We used a diamond-tipped core drill to extract a 3-mm-diameter core from a sintered disk of a zircon-based ceramic. "These cores were inserted into a ceramic holder to a maximum stacked height of $10 \mathrm{~mm}$. 'This holder was sealed in a polytetrafluroethylene capsule, and the whole assembly was loaded into a 7.5-mm zirconia rotor to form a triply contained sample (Figure 4-44). At each stage of loading, the assembly is checked for external contamination. 'The rotor was then test spun and rechecked for contamination, and once cleared it was moved to the spectrometer. In proof-of-principle tests, we cored two ceramic samples that were prepared in 1984 with $10 \mathrm{wt} \%{ }^{239} \mathrm{Pu}$ and ${ }^{238} \mathrm{Pu}$ loadings, respectively. For increased sensitivity, the ${ }^{29} \mathrm{Si}$ magic-angle spinning NMR signals were acquired with Carr, Purcell, Meiboom, and Gill echo trains.

${ }^{239} \mathrm{Pu}$ has a half-life of 24,100 years and ${ }^{238} \mathrm{Pu}$ a half-life of 87 years, thus the ${ }^{238} \mathrm{Pu}$ sample has received on the order of $10^{20} \alpha$-decays/g and the ${ }^{239} \mathrm{Pu}$ sample $-5 \times 10^{17} \alpha$-decays $/ g$. Figure $4-45$ shows the spectra of the summed Carr, Purcell, Meiboom, and Gill echoes. The ${ }^{239} \mathrm{Pu}$ sample shows very little damage, a result that agrees with data on naturally occurring $\mathrm{ZrSiO}_{4}$. 'This is an important result in terms of interpreting dose rate effects on radiation damage because this sample has received in 20 years the same dose a natural sample would have received in -500 million years. The ${ }^{238} \mathrm{Pu}$ sample, on the other hand, has become completely amorphous due to its large $\alpha$-dose, with some recrystallization of an as yet unidentified phase. 'The stage is set to produce selected samples of ${ }^{238} \mathrm{Pu}$-doped ceramics and quantitatively evaluate their radiation resistance with NMR spectroscopy.

A preliminary report of these results was presented in an invited talk by Dr. Farnan at the 2004 Experimental Nuclear Magnetic Resonance Conference (Pacific Grove, California) in April 2004. 'This conference is an annual international conference attended by $1200 \mathrm{NMR}$ spectroscopists. In addition to the paper cited in this publication, Farnan et al. 2004b was generated as a result of this work.

\section{Citations}

Farnan I, HM Cho, W J Weber, RD Scheele, NR Johnson, and AE Kozelisky. 2004a. "Magic-Angle Spinning NMR of Highly Radioactive Ceramics: ${ }^{29} \mathrm{Si}$ NMR Zircons Containing $10 \mathrm{wt} \%{ }^{239} \mathrm{Pu}$ and ${ }^{238} \mathrm{Pu}$." Presented by Ian Farnan at Experimental NMR Conference, Asilomar, California, on April 18,2004.

Farnan I, HM Cho, WJ Weber, RD Scheele, N Johnson, and AE Kozelisky. 2004b. "High-Resolution Solid-State Nuclear Magnetic Resonance Experiments on Highly Radioactive Ceramics." Review of Scientific Instruments 75(12):5232-5236.

Farnan I, and EKH Salje. 2001. "The Degree and Nature of Radiation Damage in Zircon Observed by ${ }^{29} \mathrm{Si}$ Nuclear Magnetic Resonance."Journal of Applied Physics 89(4):2084-2090.
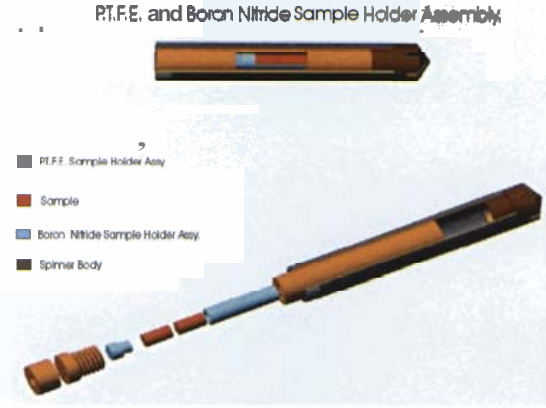

Figure 4-44. Exploded view of the triple-containment magic-anglespinning rotor for radioactive samples. The innermost capsule in this depiction is made from a boron nitride ceramic; current designs use an aluminum nitride ceramic.

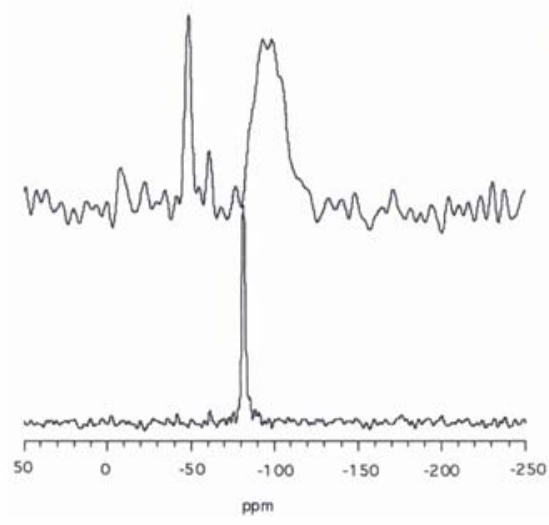

Figure 4-45. ${ }^{29}$ Si magic-angle spinning NMR spectra (sample spinning speed $=3.5 \mathrm{kHz}$ ) of a sintered zircon ceramic containing approximately 10 wt $\%{ }^{238} \mathrm{Pu}$ (top) and ${ }^{239} \mathrm{Pu}$ (bottom). The spectra were acquired with a Carr, Purcell, Meiboom, and Gill echo train. Sample masses were $75 \mathrm{mg}$ and $52 \mathrm{mg}$ for the ${ }^{239} \mathrm{Pu}$ and ${ }^{238} \mathrm{Pu}$ samples, respectively. The top spectrum reveals the highly amorphous state of the ${ }^{238} \mathrm{Pu}$ ceramic, which has received an internal a-dose more than two orders of magnitude higher than the ${ }^{239} \mathrm{Pu}$ specimen. 


\section{Detection and Characterization of ZSM-5 in a Mesoporous Host Matrix}

CA Fyfe, ${ }^{(a)}$ C Schneider, ${ }^{(a)}$ JL Bretherton, ${ }^{(a)}$ S Kaliaguine, ${ }^{(b)} \mathrm{T} D O,{ }^{(b)}$ A Nossov, ${ }^{(c)}$ and M A Springuel-Huet ${ }^{(c)}$

(a) University of British Columbia, Vancouver, British Columbia, Canada

(b) Laval University, Quebec, Canada

(c) Université Pierre et Marie Curie, Paris, France

'This is an extension of previous work at EMSL's High-Field Magnetic Resonance Facility, which included studies of ceramics and minerals as well as zeolites and resulted in six publications [Do et al. 2004; Fechtelkord et al. 2003; Fyfe et al. 2001 and 2000; Groat et al. 2003; Moorlag et al. 2005 (in press)].

Figure 4-46. Examples of zeolitic frameworks Previous research with the zeolites used high-field NMR to demonstrate that four aluminum sites were present in the catalyst zeolite-Y, instead of the three identified at lower field, and that they possessed the correct population ratio.

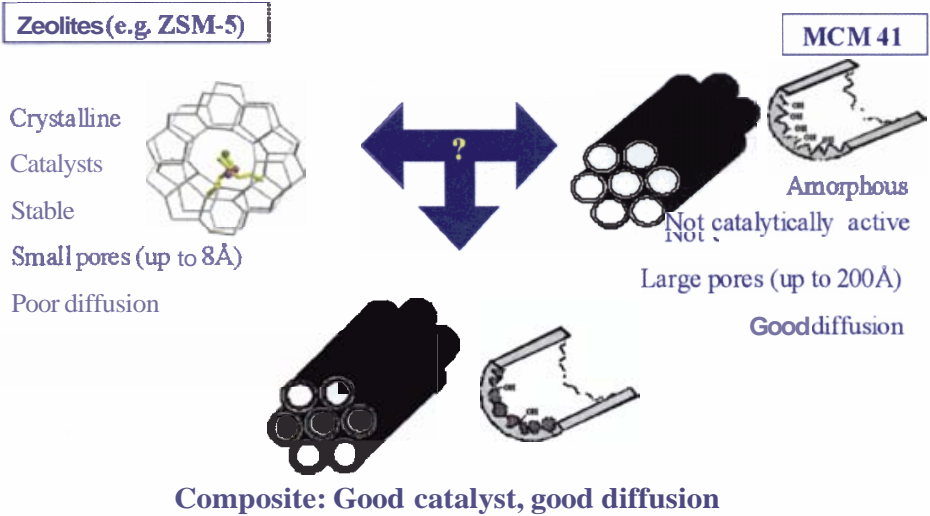

Figure 4-47. The incorporation of zeolite nanocrystals in the mesoporous structure.
The mineral studies used lithium NMR to verify in the diffraction data that there were two sites present in the amblygonite $\left(\mathrm{LiAlPO}{ }_{4} \mathrm{~F}\right)$ montebrasite $\left(\mathrm{LiAlPO}{ }_{4} \mathrm{OH}\right)$ solid solution; to measure internuclear distances in this solution; and to determine that the sites were randomly distributed in the material, which diffraction measurements could not determine.

Zeolites are very open frameworks of aluminosilicates or silicates composed of corner- and edge-sharing $\mathrm{SiO}_{4}{ }^{4-}$ and $\mathrm{AlO}_{4}{ }^{5-}$ tetrahedra. 'Ihey contain regular systems of cavities and channels of molecular dimensions that control the uptake of organic molecules in terms of shape and size selectivity (Figure 4-46). They are powerful acid catalysts, but their small pores limit diffusion. It has been shown recently that the coating of protozeolitic nanoclusters onto the surface of preformed mesostructured aluminosilicates can greatly improve their hydrothermal stability and acidity, both essential for catalysis (Figure 4-47). Although the change of pore size and volume as well as Fourier-transform infrared observations indicated the location of the zeolite nanoclusters in the mesopore channels, XRD diagrams failed to prove the presence of zeolite crystals in the coated material. Ultrahigh-field (17.6 tesla) ${ }^{27} \mathrm{Al}$ magic-angle-spinning and multiple quantum magic-angle spinning NMR was used to detect the zeolite nanocrystals and quantify the multiple aluminum environments in these materials. A complete account is given in Do et al. 2004.

Figure 4-48a shows the various aluminum environments detected by ${ }^{27} \mathrm{Al}$ magic-angle spinning NMR at 17.6 tesla. 'Ihe parent sample shows two broad peaks (one tetrahedral and the other octahedral) characteristic of amorphous 
materials and the calcined zeolite-coated samples show two additional sharper peaks consistent with the chemical shift values of the corresponding zeolite. 'The broadening of these peaks, compared to the ones obtained from perfectly crystalline zeolites, results from their being in a less ordered environment. As Figure 4-48b shows, it was possible to discriminate the higher degree of ordering of the zeolite (longer T2) from the amorphous mesoporous framework (shorter $\mathrm{T} 2$ ) through a series of spin echo experiments. ${ }^{27} \mathrm{Al}$ multiple quantum magicangle spinning NMR confirms these results, as only two partially resolved signals are observed and assigned to the tetrahedral aluminum sites in the zeolite (Figure 4-48c).

Figure 4-48.

The use of ultrahigh field ${ }^{27} \mathrm{Al}$ NMR to discriminate the nanozeolite from the mesostructure.

a) Single pulse

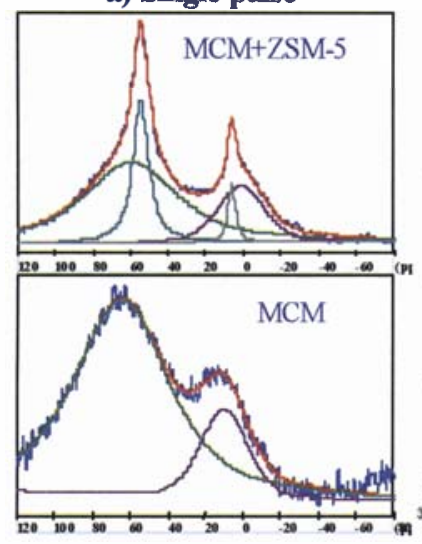

b) Spin Echo

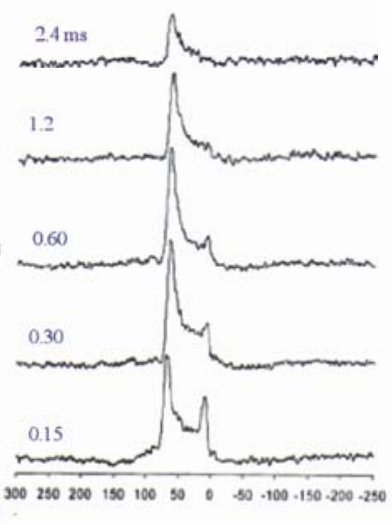

c) 3QMAS

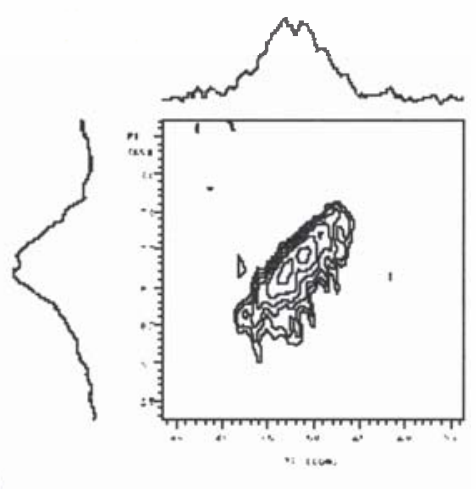

Ultrahigh-field ${ }^{27} \mathrm{Al}$ magic-angle spinning and multiple quantum magicangle spinning NMR proved to be extremely useful tools for the detection of zeolite nanoclusters and the different aluminum environments in nanozeolite/ mesoporous aluminosilicate composites, which-are impossible to detect by conventional XRD techniques.

\section{Citations}

Do T, A Nossov, MA Springuel-Huet, C Schneider,JL Bretherton, CA Fyfe, and S Kaliaguine. 2004. "Zeolite Nanoclusters Coated onto the Mesopore Walls of SBA-15." Journal of the American Chemical Society 126(44):14324-14325.

Fechtelkord M, H Behrens, F Holtz, J Bretherton, CA Fyfe, LA Groat, and M Raudsepp. 2003. "Influence of Fluorine Content on the Composition of Al-rich Synthetic Phlogopite: Probing the Structural Arrangement of Fluorine and Hydroxyl Anions in the Brucite Layers by ${ }^{27} \mathrm{Al}$ MQMAS and ${ }^{1} \mathrm{H} /{ }^{19} \mathrm{~F}-{ }^{27} \mathrm{Al}$ HETCOR and REDOR Experiments."American Mineralogist 88(1):47-53.

Fyfe CA, JL Bretherton, and LY Lam. 2001. "Solid-State NMR Detection, Characterization and Quantification of the Multiple Aluminum Environments in USY Catalysts by ${ }^{27} \mathrm{Al}$ MAS and MQMAS Experiments at Very High Field." Journal of the American Chemical Society 123(22):5285-5291. 


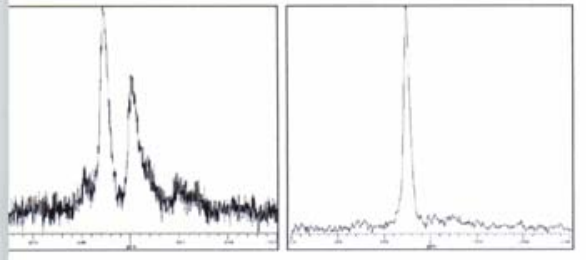

Figure 4-49. (Left): Aluminum NMR magic-angle spinning spectra for siliceous sinter from the Kamchatka Peninsula. Significant quantities of both tetrahedral and octahedral aluminum are present. (Right): Aluminum NMR magic-angle spinning spectra for siliceous sinter from Yellowstone National Park. This ${ }^{27}$ Al spectrum shows only the tetrahedral aluminum species present.
Fyfe CA,JL Bretherton, and LY Lam. 2000. "Detection of the "Invisible Aluminum" and Characterization of the Multiple Aluminum Sites in Zeolite USY by High-Field Solid-State NMR." Chemical Communications 17:1575-1576.

Groat LA, BC Chakoumakos, CM Hoffman, H Morell, CA Fyfe, D Brouwer, and AJ Schulz. 2003. "The Amblygonite $\left(\mathrm{LiAlPO}_{4} \mathrm{~F}\right)$ Montebrasite $\left(\mathrm{LiAlPO}{ }_{4} \mathrm{OH}\right)$ Solid Solution: A Single Crystal Neutron Diffraction and ${ }^{6} \mathrm{Li}$ MAS-NMR Spectroscopic Study." American Mineralogist 88(1):195-210.

Moorlag C, QYang, T Troczynski, J Bretherton, and CA Fyfe. 2005. "Aluminum Phosphates Derived from Alumina and Alumina Sol-Gel Systems." Journal of the American Ceramic Society (in press).

\section{Chemical Signatures in Thermal Springs: Atomic-Level Structure of Silicon and Aluminum in Natural and Synthetic Minerals}

N Hinman, ${ }^{(a)}$ A Tenesch, ${ }^{(a)} \mathrm{H}$ Cho, ${ }^{(b)}$ and S Burton ${ }^{(b)}$

(a) University of Montana, Missoula, Montana

(b) Pacific Northwest National Laboratory, Richland, Washington

Many factors drive chemical change in geothermal systems. Long-term effects include climatic change; change in the location, quantity, and rate of release of heat; and volcanic processes. For example, climatic processes affect the amount of water circulating through the system and, therefore, the amount of heat released as steam and liquid water. Mid-term effects include seasonal processes and human impacts; for example, interactions between shallow groundwater and deeper thermal water can affect chemistry of the water in surface springs. Shortterm effects include photochemical and photosynthetic processes; for example, reduced forms of iron are detected in mid-day because of photochemical processes, whereas the by-products of such reactions apparently reduce sulfide concentrations. 'These changes result from changes in $\mathrm{pH}$, redox conditions, and solution composition, and can impart chemical signatures in deposits formed in such springs.

What signatures are likely to be preserved in the geological record? Because chemical changes can affect the $\mathrm{pH}$ and redox conditions of thermal springs, it makes sense to pursue signatures of elements affected by these factors. Although many minor elements are affected by these changes, in this study we focus on changes in major element speciation, specifically, the concentrations and forms of aluminum and silicon in thermal springs of different compositions. Waters from an unnamed geyser in Shoshone Geyser Basin (Yellowstone National Park, Wyoming) and from the Trinoi Geyser (Krontoskii Reserve, Kamchatka, 
Russia) were selected to represent different spring water chemistries that might result from changes over a long time period or that might result from original differences in host rock composition.

NMR spectra acquired from instruments at EMSL show differences in the atomic environment of aluminum between waters from the unnamed U.S. geyser and the Trinoi Geyser (Figure 4-49). Water from the Trinoi Geyser shows significant quantities of both tetrahedrally coordinated aluminum and octahedrally coordinated aluminum. Water from the unnamed U.S. geyser has no octahedrally coordinated aluminum. 'The differences in the silicon environment in waters from the Trinoi and Pork Chop geysers are not as pronounced. Solid-state silicon-NMR shows two distinct silicon environments. Results from cross-polarizationexperiments (Figure 4-50) show silicon bridging to two other silicon atoms via oxygen (Q2) and to three other silicon atoms via oxygen (Q3). Sinters from both Yellowstone National Park and the Krontoskii Reserve are a mix of these two silicon types.

Such differences in the aluminum's atomic environment may be attributed to effects of solution chemistry on the speciation of dissolved forms of aluminum. Speciation affects the rate and properties of solid deposition and, therefore, is the likely explanation in the observed differences in NMR spectra. Springwater chemistry, therefore, affects the properties of solids formed there and may yield evidence of past changes. This work has been important for disclosing that NMR is a useful tool for geological work.

As a consequence of these earlier results, silicon-aluminum transfer of population by double resonance (TRAPDOR) experiments were conducted to determine the proximity of aluminum and silicon in a siliceous sinter matrix. Siliconoxygen-aluminumbridges should be detectable by monitoring the silicon NMR frequency while pulsing the aluminum NMR frequency. If a reduction in signal occurs during the TRAPDOR pulsing experiments, then aluminum is close enough to silicon to influence the silicon nucleus. The NMR frequencies of aluminum and silicon are sufficiently similar that the experiment might not work. For this reason, feldspar was used as a known, with little, if any, TRAPDOR effect observed. However, a significant signal was observed in the siliceous sinter from Trinoi Geyser (Figure 4-51). It is difficult to draw conclusions about aluminum effects on silicon on the basis of only one sample. However, this work demonstrated the success of the silicon-aluminum TRAPDOR experiment. More TRAPDOR experiments have been run since this initial test of the experiment and we are currently working on a manuscript with the results.

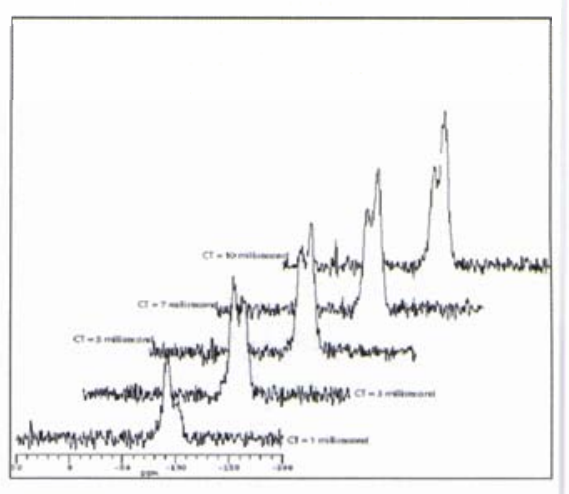

Figure 4-50. Cross-polarization magic-angle spinning silicon NMR spectra for siliceous sinter from the Kamchatka Peninsula. The array illustrates the affect of increasing cross-polarization contact times on the observation of Q2and Q3.

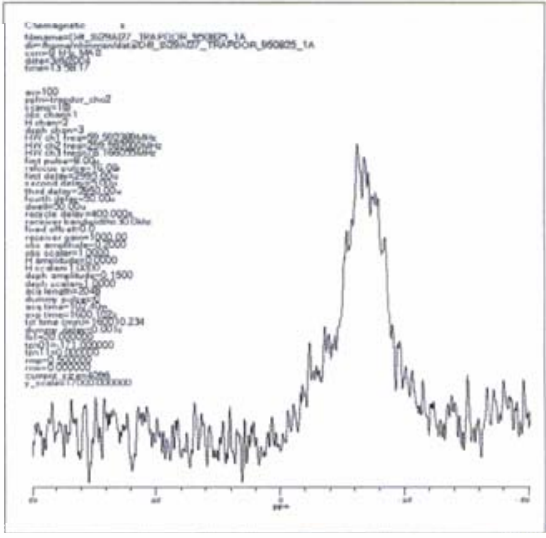

Figure 4-51. Silicon TRAPDOR spectrum of a siliceous sinter from Trinoi Geyser calculated from the difference between silicon spectra collected with and without pulsing at aluminum frequency. In the silicon TRAPDOR experiment, the difference between the two spectra represents silicon nuclei that are influenced by nearby aluminum nuclei. 


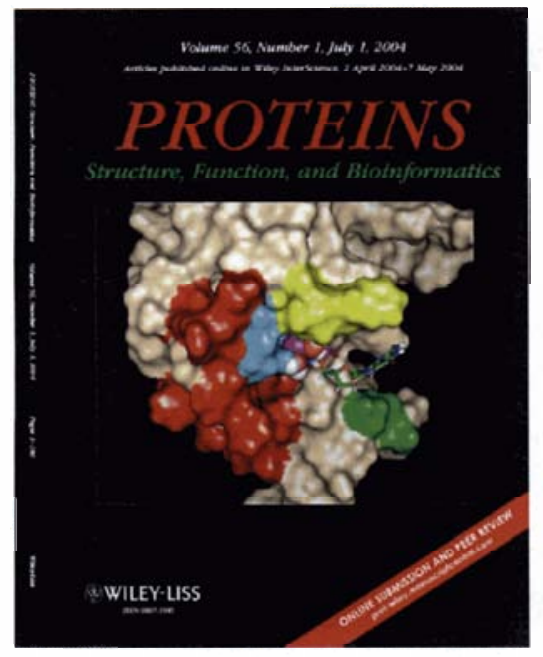

Figure 4-52. Our structure of the Nudix hydrolase DR0079 was featured on a cover of Proteins. Shown is a proposed model for the binding of a generic substrate (nucleoside and diphosphate, atomic colors) and hydrated, divalent metal cation ion (pink) to the solution structure of DR079.

\section{NMR Spectroscopic Investigations of Nudix Hydroylases from the Extremely Radiation-resistant "Superbug" Deinococcus Radiodurans}

GW Buchko, ${ }^{(a)}$ S. Ni, ${ }^{(a)}$ SR Holbrook, ${ }^{(b)}$ and M A Kennedy ${ }^{(a)}$

(a) Pacific Northwest National Laboratory, Richland, Washington

(b) Lawrence Berkeley National Laboratory, Berkeley, California

The bacterium Deinococcus radiodurans is often called a "superbug" because of its ability to survive under conditions that are lethal to most other organisms. It is able to withstand high doses of ionizing and ultraviolet radiation, physical and chemical DNA-damaging agents, and is highly resistant to desiccation. 'The molecular bases for these unusual properties are unknown. Consequently, there is considerable interest in identifying the proteins responsible for the "superbug" properties of $D$. radiodurans and understanding how they work. One hypothesis is that the resistance to radiation and to other DNA-damaging agents (including hydrogen peroxide and mitomycin C) may be due to an unusually high number of surveillance and housecleaning enzymes called Nudix hydroylases.

Nudix proteins are widely distributed among species, having been found in the genomes of the three kingdoms and in the genomes of organisms as diverse as viruses and humans. 'Ihey are identified by the consensus sequence $\mathrm{GX}_{5} \mathrm{EX}_{7} \mathrm{REUXEEXGU}$ (where $\mathrm{U}=\mathrm{I}$, $\mathrm{L}$, or $\mathrm{V}$ and $\mathrm{X}=$ any amino acid) that forms part of the catalytic site for the hydrolysis of a NUcleoside DIphosphate linked to some other moiety, $\mathrm{X}$. The prototypical member of this family is the Escherichia coli MutT protein that catalyzes hydrolysis of nucleotide triphosphates with a preference for 8-oxo-dGTP. The primary functions of Nudix proteins are to sanitize the cell by reducing the level of potentially mutagenic and/or toxic compounds and the accumulation of biochemicalintermediates. While more than 1200 putative Nudix proteins have been identified in genomes on the basis of the Nudix consensus sequence, few Nudix protein structures have been determined. For those few structures that have been determined, beyond the conserved cataylic core there is a considerable variation in the peripheral structure and oligomerization state. Therefore, we have targeted the 23 hypothetical Nudix proteins for structure determination in order to help identify their cellular functions and to relate their structural diversity to functional diversity. Using the NMR facilities at EMSL, we collected data that allowed us to determine the solution structure for the hypothetical $D$. radiodurans Nudix protein DR0079, a 171 residue, $19.3 \mathrm{kDa}$ protein.

Our structure for DR0079 was featured on the cover of the journal Proteins (Figure 4-52). Figure 4-53 shows a cartoon representation of the protein's structure generated by Molscript. The protein contains eight $\boldsymbol{\beta}$-strands and three 
a-helices organized into three subdomains: an N-terminal 6-sheet (1-34), a central Nudix core (35-140), and a C-terminal helix-turn-helix motif (141-171). 'Ihe Nudix core and the C-terminal helix-turn-helix motif form the fundamental fold common to the Nudix family, a large mixed $\beta$-sheet sandwiched between a-helices. While Figure 4-52 shows that is possible to model a generic substrate into the location of the likely active site, it is not possible to predict the exact substrate for DR0079 based on the structure. Finding the biochemical substrate for DR0079 will require further experimentation to determine if DR0079 plays a "super function" in this "superbug."

\section{The following publications have been generated as part of this work:}

Buchko GW, S Ni, SR Holbrook, and M A Kennedy. 2004. "Solution Structure of Hypothetical Nudix Hydrolase DR0079 from Extremely-radiation Resistant Deinococcus Radiodurans Bacterium." Proteins 56:28-39.

Buchko, GW, S Ni, SR Holbrook, and M A Kennedy. 2003. " ${ }^{1} \mathrm{H},{ }^{13} \mathrm{C}$, and ${ }^{15}$ N NMR Assignments of the Hypothetical Nudix Protein DR0079 from the Extremely Radiation-resistant Bacterium Deinococcus Radiodurans."Journal $₫$ Biomolecular NMR 25:169-170.

\section{NMR Structural Investigations of the Breast Cancer Susceptibility Protein, BRCAI}

P Brzovic, ${ }^{(a)}$ DW Hoyt, ${ }^{(b)}$ and RE Klevit ${ }^{(a)}$

(a) University of Washington, Seattle, Washington

(b)W.R. Wiley Environmental Molecular Sciences Laboratory, Richland, Washington

'Ihe breast and ovarian cancer tumor suppressor protein, BRCAl is central to a number of fundamental cellular pathways. Its absence during embryogenesis is lethal. Loss of function in proliferating breast or ovarian epithelial cells can result in the development of cancer. BRCAl has been shown to function in processes such as the cellular response to DNA damage, homologous recombination, transcriptional regulation, and, more recently, ubiquitination. Protein ubiquitination provides a powerful regulatory mechanism for controlling pathways that include cell-cycle progression, transcriptional regulation, and responses to DNA damage. The importance of ubiquitin to cellular viablity is underscored by the award of the 2004 Nobel Prize in Chemistry for research into the function of ubiquitination at the molecular level.

$\mathrm{BRCAl}$ is a large and complicated protein which is undoubtedly comprised of a multiplicity of functional domains. A growing body of literature suggests that BRCAl interacts with at least 30 different macromolecules to accomplish its diverse functional roles. To date, our structural work has focused on the $\mathrm{N}$-terminal RING domain of BRCAl in complex with the N-terminal RING

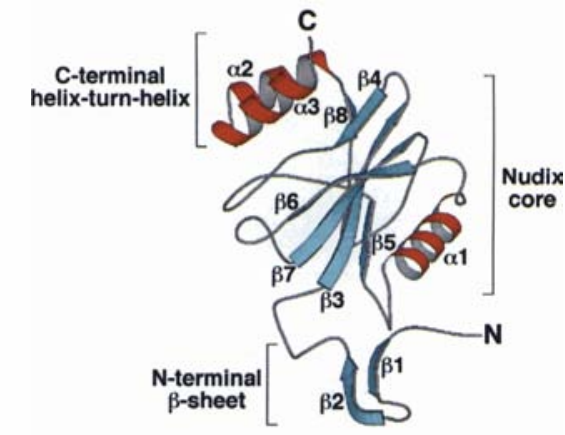

Figure 4-53. Ribbon representation of the solution structure of DR079 (PDB 1Q27). The structure was determined using NMR-based distance geometry methods that included the use of residual dipolar coupling restraints. The three a-helices and eight $\beta$-strands are labeled. Alpha helix 1 contains the Nudix box. 


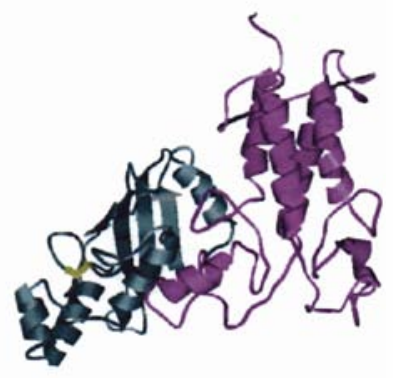

Figure 4-54. Structure of the BRCA1-BARD1 heterodimer (magenta) in complex with UbcH5c (blue-green). The UbcH5c active site cysteine is highlighted in yellow.

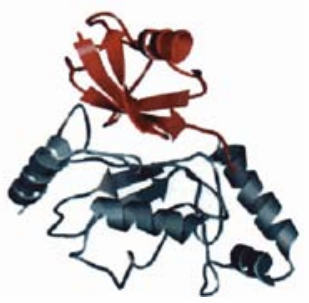

Figure 4-55. Structure of the noncovalent complex formed between $\mathrm{UbcH} 5 \mathrm{c}$ and Ubiquitin. Ubiquitin (red) binds to the exposed $\boldsymbol{\beta}$-sheet region of UbcH5c (blue-green). The active site of $\mathrm{UbcH} 5 \mathrm{C}$ is on the opposite side of the molecule. domain of BARD1. Of particular interest is the recent demonstration that BRCA1 RING functions as a ubiquitin ligase and interaction with the BARD1 RING is obligatory for this activity. As E3-ligases, RING domains are thought to facilitate the specificity of ubiquitination reactions by forming a multiprotein complex, binding both a ubiquitin conjugating enzyme (E2) covalently activated with ubiquitin and specific proteins targeted for ubiquitination. Cancer predisposing mutations found in the BRCAl RING domain have been found to interfere with its ability to function as a ubiquitin ligase. Thus, our research at EMSL is poised to study two critical cellular pathways at the molecular level.

This system provides a unique opportunity for studying protein-protein interactions by NMR. It involves characterizing the structures and interactions between at least four different protein components: BRCA1, BARD1, an E2 (UbcHSc or UbcH7) and ubiquitin (Ub). The molecular weight of the fully assembled complexes approaches $60 \mathrm{kD}$. In previous years, we have been able to collect a great deal of data on the individual components of this system. During the last year, data collected on the 600-, 800-, and 900-MHz NMR spectrometers have allowed us to develop a model of this multiprotein complex.

The first piece of the puzzle was determination of the solution structure of the BRCA1-BRCA1 RING domain heterodimer. Subsequently, we solved the NMR solution structure of $\mathrm{UbcH} 5 \mathrm{c}$. With these two structures in hand, we were able to investigate the complex formed between the BRCA1-BARD1 RING heterodimer and UbcH5c (Figure 4-54). Although other RING proteinE2 structures have been reported, this complex is catalyticallycompetent and the two proteins appear to associate in a manner substantially different from previous reports. These studies help to delineate factors that govern the specificity of RING(E3)-E2 protein interactions.

Secondly, our studies at EMSL's High-Field Magnetic Resonance Facility uncovered a noncovalent binding site for $\mathrm{Ub}$ on a surface of $\mathrm{UbcHSc}$ located on a surface far removed from the active site. A single mutation in UbcHSc that disrupts this interaction is sufficient to eliminate BRCAl-directed polyubiquitin chain formation in vitro. Though the affinity of this site for $\mathrm{Ub}$ is low $(\mathrm{Kd}-300 \mu \mathrm{M})$ data collection on both $800-$ and $900-\mathrm{MHz}$ instruments at EMSL has been critical for calculating the structure of the $\mathrm{UbcH} 5 \mathrm{c}-\mathrm{Ub}$ structure (Figure 4-55.)

Recently, we have been able to generate and collect preliminary spectra on the activated $\mathrm{UbcH} 5 \mathrm{c} \sim \mathrm{Ub}$ covalent complex. In this complex, the $\mathrm{C}$-terminus of $\mathrm{Ub}$ is covalently attached to the active site cysteine residue of UbcHSc via the formation of a thiolester bond. Surprisingly, with the exception of the extreme $\mathrm{C}$-terminal tail of $\mathrm{Ub}$, there are no other apparent interactions between $\mathrm{UbcH} \mathrm{Hc}$ and the attached Ub moiety.

These accomplishments allow us to build a model of an active BRCA1/BARD1 E3 ubiquitin ligase complex (Figure 4-56). This is particularly exciting since these complexes have previously not been amenable to detailed structural characterization. This work was made possible by the high-field NMR instrumentation available at EMSL. We anticipate that during the coming year, 
these studies will help us further understand the complicated set of interactions between BRCA1 and its partner proteins.

\section{The following publications have been generated as part of this research:}

Brzovic PS, J O'Keeffe, DE Christensen, DW Hoyt, and RE Klevit. 2004. "Structure of a Non-covalent Ubch5c-Ub Complex Required for BRCA1-directed Polyubiquitinylation." (In preparation.)

Brzovic PS, JR Keeffe, H Nishikawa, K Miyamoto, D Fox, M Fukuda, T Ohta, and RE Klevit. 2003. "Binding and Recognition in the Assembly of an Active BRCA1BARD1 Ubiquitin Ligase Complex." Proceedings of the National Academy of Sciences 100:5646-5651.

Brzovic PS, P Rajagopal, DW Hoyt, MC King, and RE Klevit. 2001. "Structure of a BRCA1-BARD1 Heterodimeric RING-RING Complex." Natural Structural Biology 10:833-837.

\section{NMR Spectroscopic Investigation of Human Carbonic Anhydrase Points to Alternative Mechanism of Action}

AS Lipton, ${ }^{(a)}$ B Heck, ${ }^{(a)}$ JA Sears, ${ }^{(b)}$ and PD Ellis ${ }^{(a)}$

(a) Pacific Northwest National Laboratory, Richland, Washington

(b)W.R. Wiley Environmental Molecular Sciences Laboratory, Richland, Washington

Carbonic anhydrase catalyzes a deceptively simple chemical reaction - the hydration of $\mathrm{CO}_{2}$ to yield bicarbonate and a proton: $\mathrm{CO}_{2}+\mathrm{H}_{2} \mathrm{O}=\mathrm{HCO}_{3}{ }^{-}+$ $\mathrm{H}^{+}$. Further, it is the first enzyme recognized to have a biological function for $\mathrm{Zn}^{2+}$, which was postulated as the activation of bound $\mathrm{H}_{2} \mathrm{O}$. Abundant in all mammalian tissues, plants, algae, and bacteria, carbonic anhydrase is perhaps the most studied metalloprotein with more than 180 crystal structures in the protein database. Scientists at PNNL recently found evidence that a commonly held mechanism of action for human carbonic anhydrase may not be correct. Instead, the new data are consistent with an alternative mechanism proposed in Merz et al. 1989, and were generated using novel low-temperature solid-state NMR spectroscopy, which allowed direct observation of the $\mathrm{Zn}^{2}+$ in carbonic anhydrase.

Originally, this research began as part of efforts to calibrate the spectroscopy developed at EMSL; however, during the process, the scientists discovered that assumptions about how this enzyme works were incorrect. The spectroscopy data showed that more research is warranted to understand the mechanisms of carbonic anhydrase. In addition, EMSL now has a powerful tool to probe otherwise very difficult states in this class of metalloprotein.

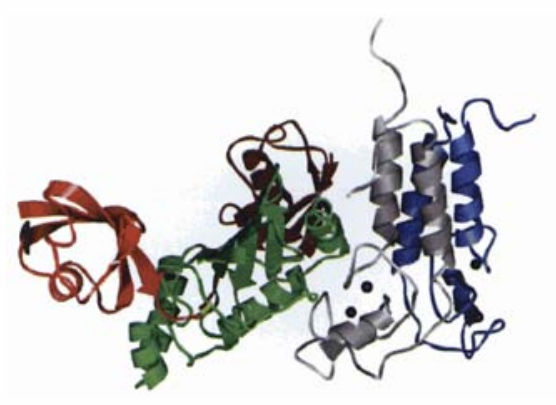

Figure 4-56. Assembly of an active ubiquitin ligase complex involving BRCA1(gray)/ BARD1(blue), UbcH5c(green), and $\mathrm{Ub}(\mathrm{red})$. 
(a)

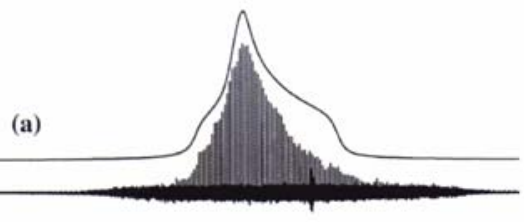

(b)

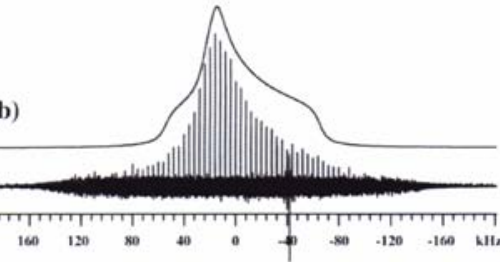

Figure 4-57. Low-temperature (10 K) solid-state ${ }^{67}$ Zn NMR spectra of carbon anhydrase at (a) $\mathrm{pH} 5$ and (b) $\mathrm{pH}$ 8.5. Above each experimental spectrum is a simulation of the spikelet envelope. At $\mathrm{pH} 5$, the extracted value of $\mathrm{Cq}$ is $9.6 \mathrm{MHz}$, whereas at $\mathrm{pH} 8.5$ the value is $10 \mathrm{MHz}$.
The most active form of carbonic anhydrase has very fast reaction rates approaching $-106 / 8$. As a result, innumerable studies have been conducted directed at this protein's action mechanism. The accepted reaction mechanism holds that the water ionizes (rate-limiting step) to yield a bound hydroxide that then adds to the nearby $\mathrm{CO}_{2}$, resulting in a $\mathrm{Zn}^{2+}$ - bound bicarbonate. 'The presence of bound water and fast catalytic rates together presents a problem that up to now could not be explained. Simple arguments would predict that the fastest that carbonic anhydrase could turn over would be at rates on the order of $104 / 8$.

The principal observable in a solid-state ${ }^{67} \mathrm{Zn}$ NMR experiment is the quadrupole coupling constant, $\mathrm{Cq}$. The ${ }^{67} \mathrm{Zn} \mathrm{Cq}$ values are sensitive to changes in structure and bonding associated with water or hydroxide. Figure 4-57 shows data collected using EMSL's 18.8-tesla NMR spectrometer, illustrating that the ${ }^{67} \mathrm{Zn} \mathrm{NMR} \mathrm{measurement} \mathrm{is} \mathrm{independent} \mathrm{of} \mathrm{pH}$ over the range of 5 to 8.5.

'The two spectra are essentially the same. At pH 5, the $\mathrm{Zn}^{2}+$ should be coordinated by $\mathrm{H}_{2} \mathrm{O}$, and as a result, the ${ }^{67} \mathrm{Zn}$ NMR spectrum is expected to be three to five times broader than the spectrum at $\mathrm{pH}$ 8.5. This is clearly not the case; however, these data are consistent with $\mathrm{OH}$ - being bound to $\mathrm{Zn}^{2}+$, not $\mathrm{H}_{2} \mathrm{O}$ over the entire $\mathrm{pH}$ range investigated and as modeled by molecular theory. This observation is contradictory to the accepted mechanism. However, it is consistent with an alternative mechanism proposed by Merz et al. 1989 . Furthermore, these data also provide an explanation for the issues associated with the turnover rates for carbonic anhydrase and point to an important complementary aspect of NMR methods to XRD, namely the sensitivity of the NMR parameters to the presence or absence of protons. Moreover, this work demonstrates the impact zinc spectroscopy can have with respect to delineating the structure and action mechanism of this important class of metalloproteins.

As a byproduct of this research, we will gain an understanding of the structure/ function relationships for metalloproteins in general. This can have applicability for other metals such as mercury, magnesium, lead, and/or iron. 'This can also have implications for bioremediation or heavy-metal trafficking within microbial communities. 'The first step in understanding how to fine-tune a system or how metals poison a system (i.e., co-carcinogenesis) is comprehension of the native systems, which is the work we are undertaking.

The research described above is one part of a research effort to facilitate the direct observation of dilute solid spins within metalloproteins. Work in this area has been enhanced greatly by the collaborations with users Gerald Parkin from Columbia University and Dan Reger from the University of South Carolina, who provided model compounds containing ${ }^{67} \mathrm{Zn}$, and Evan Kantrowitz from Boston College, who contributed ${ }^{67} \mathrm{Zn}$ metallo proteins like alkaline phosphatase. Critical to this project has been our experimental improvements to increase sensitivity. Additional papers (Lipton et al. 2004a and b; Lipton et al. 2003; Lipton et al. 2002a and b; Lipton et al. 2001a and b; Larsen et al. 1998a, b, and c; Larsen et al. 1997; and Vosegaard et al. 1997) provide more details pertaining to observation of solid-state zinc and the methods that make this research possible. 


\section{Citations}

Larsen FH, HJ Jakobsen, PD Ellis, and NC Nielsen. 1998a. "Determination of Molecular Dynamics by ${ }^{2} \mathrm{H}$ QCPMG Solid-State NMR Spectroscopy." Chemical Physics Letters 292(4-6):467-473.

Larsen FH, HJ Jakobsen, PD Ellis, and NC Nielsen. 1998b. "High-Field QCMPGMAS NMR of Half-Integer Quadrupolar Nuclei With Large Quadrupole Couplings." Molecular Physics 95(6):1185-1195.

Larsen FH, HJ Jakobsen, PD Ellis, and NC Nielsen. 1998c. "QCPMG-MAS NMR of Half-Integer Quadrupolar Nuclei." Journal of Magnetic Resonance 131:144-147.

Larsen FH, HJ Jakobsen, PD Ellis, and NC Nielsen. 1997. "Sensitivity-Enhanced Quadrupoloar Echo NMR of Half-Integer Quadrupolar Nuclei. Magnitudes and Relative Orientation of Chemical Shielding and Quadruplar Coupling Constants." Journal of Physical Chemistry A 101(46):8597-8606.

Lipton AS, RW Heck, JA Sears, and PD Ellis. 2004a. "Low Temperature Solid-State NMR.Experiments of Half-Integer Quadrupolar Nuclides: Caveats and Data Analysis." Journal of Magnetic Resonance 168:66-74.

Lipton AS, RW Heck, and PD Ellis. 2004b. "Zinc Solid-State NMR Spectroscopy of Human Carbonic Anhydrase. Implications for the Enzymatic Mechanism."Journal of the American Chemical Society 126(14):4735-4739.

Lipton AS, C Bergquest, G Parkin, and PD Ellis. 2003. "Solid State ${ }^{67}$ Zn NMR Spectroscopic and ab initio Molecular Orbital Calculations on a Synthetic Analogue of Carbonic Anhydrase." Journalof the American Chemical Society 125(13):3768-3772.

Lipton AS, T Wright, MK Bowman, DL Reger, and PD Ellis. 2002a. "Solid State ${ }^{67} \mathrm{Zn}$ NMR Spectroscopy in Bioinorganic Chemistry. Spectra of 4-and 6-Coordinate Zinc Pyrazolylborate Complexes Obtained by Management of Proton Relaxation Rates with a Paramagnetic Dopant." Journal of the American Chemical Society 124(20):5850-5860.

Lipton AS, MD Smith, RD Adams, and PD Ellis. 2002b. "Solid State ${ }^{67} \mathrm{Zn}$ NMR Spectroscopy in Bioinorganic Chemistry. ${ }^{67} \mathrm{Zn}$ Solid-State and Single Crystal NMR Spectroscopy and X-ray Crystal Structure of Zinc Formate Dihydrate." Journalof the American Chemical Society 124(3):410-414.

Lipton AS, JA Sears, and PD Ellis. 2001a. "A General Strategy for the NMR Observation of Half-Integer Quadrupolar Nuclei in Dilute Environments." Journal 6 Magnetic Resonance 151:48-59.

Lipton AS, G W Buchko,JA Sears, M A Kennedy, and PD Ellis. 2001b. “67Zn SolidState NMR of the Minimal DNA Binding Domain of Human Nucleotide Excision Repair Protein XPA." Journal of the American Chemical Society 123(5):992-993.

Merz KM, R Hoffmann, and MJS Dewar. 1989. "The Mode of Action of Carbonic Anhydrase." Journal of the American Chemical Society 111(15):5636-5649.

Vosegaard T, FH Larsen, HJ Jakobsen, PD Ellis, and NC Nielsen. 1997. “SensitivityEnhanced Multiple-Quantum MAS NMR of Half-Integer Quadrupolar Nuclei." Journal of the American Chemical Society 119(38):9055-9056. 


\section{Application of High-Field Solid-State NMR Spectroscopy to Clays Contaminated with Simulated Hanford Tank Waste}

GM Bowers, ${ }^{(a)}$ S Choi, (b) J Chorover, ${ }^{(b)}$ GS Crosson, ${ }^{(a)}$ and KT Mueller ${ }^{(a)}$

(a) Pennsylvania State University, University Park, Pennsylvania

(b) University of Arizona, Tucson, Arizona

An understanding of radio-cation mobility in the lithosphere and hydrosphere is necessary to predict the environmental fate of nuclides released from leaking storage tanks. The types of minerals present in the soil, their cation exchange capacities, and their respective affinities for various cations play an important role in determining cation transport. Clay minerals exposed to Hanford Site tank waste under near-field conditions have been shown to undergo mineral transformation reactions that produce tetrahedral aluminosilicate phases, introducing new mineral phases to the soils and complicating current transport models with mineral dissolution and formation kinetics. In order to model Hanford soil chemistries, kaolinite clay samples similar to that found at Hanford have been exposed to a simulated tank waste leachate solution $\left(0.05 \mathrm{M} \mathrm{Al}(\mathrm{OH})_{4}{ }^{-}, 2 \mathrm{M} \mathrm{Na}^{+}, 1 \mathrm{M} \mathrm{NO}_{3}{ }^{-}, \mathrm{pH}\right.$ near 14 , various concentrations of $\mathrm{Cs}^{+}, \mathrm{Sr} 2^{+}, \mathrm{I}-$ ), with the goal of investigating precipitate formation and identifying precipitates that sequester cesium, strontium, and iodine. For these investigations, solid-state NMR and complementary analytical techniques are being used.

Preliminary ${ }^{27} \mathrm{Al}$ solid-state NMR spectra at 7 tesla show the growth of an asymmetrical peak near 60 pprn when kaolite clay is exposed to simulated tank waste leachate. 'This peak is consistent with the formation of tetrahedral aluminum sites similar to those found in zeolites; the peak near 4 pprn originates from the six coordinate aluminum found in untreated clay. "The asymmetry of the new peak suggests that there are multiple sites present, but studies at higher fields will be needed to resolve the issue. "The peak near $60 \mathrm{pprn}$ sharpens as the field is increased and the best resolution has been obtained from the 17.6-tesla spectrometer at EMSL. High-field NMR provides evidence that the broad peak is due to a single chemical environment, but additional features at -55 pprn and -65 pprn are present that are not visible at lower fields, due to their lower resolution and sensitivity. 'These features indicate that there are additional chemical environments present. By varying the ratio of silicon/aluminum in synthetic colloidal silica samples, the relative abundance of these new environments, as denoted by the 55 pprn and 65 pprn peaks, can be measured, as well as how changing reaction conditions might affect the ratio of these phases. Data at 9.4 tesla was not sufficiently resolved for this analysis. 'These peaks, and therefore the structures that give rise to them, are significantly reduced when the silicon/aluminum ratio is $2: 1$ during the reactions, but they are clearly present when the ratio is 1:1. Temperature during the reactions did 
not affect the relative intensities. Clearly, adding simulated tank waste leachate to clay soils affects the chemistry of the soils and, therefore, the speed at which cesium and strontium cations can move through the soil. It would be better, though, to observe the cesium and strontium directly by NMR to determine how they are interacting with the soil.

The interactions of strontium with clay minerals and zeolites are not well understood and must be researched in order to construct accurate models for predicting the environmental fate of radioactive strontium-90 released from sites such as Hanford. However, there is only one NMR active isotope of strontium $\left({ }^{87} \mathrm{Sr}\right)$ and the direct study of strontium with solid-state NMR is experimentally challenging. Strontium-87 has similar chemistry to ${ }^{90} \mathrm{Sr}$ and is a quadrupolar nucleus $(I=-9 / 2)$ with a low natural abundance ( -7 percent), a low gyromagnetic ratio ( $\mathrm{y}=-1.163 \times 10^{7} 1 / \mathrm{T}_{-}$s), and large quadrupolar coupling constants (14$25 \mathrm{MHz}$ ). This combination of properties indicates that the highest possible magnetic fields must be used for these studies. The 900-MHz system (21.1 tesla) at EMSL is ideal for this work, especially coupled with modern techniques to enhance sensitivity. Figure 4-58 shows data from the mineral strontianite, or strontium chloride, under different conditions. It is evident that there is a substantial improvement in signal to noise by going from 11.7 tesla to 21.1 tesla, and that there is an additional significant increase in signal to noise by using the Quadrupole Carr-Purcell Meiboom Gill (QCPMG) data collection sequence. The net increase in signal to noise from spectrum A to $C$ is over 800 fold. Since signal-to-noise increases with the square root of the number of scans, this results in a 640,000 fold savings in time. 'This demonstrates that strontium can be studied routinely at 21.1 tesla in pure materials. As part of our ongoing studies at PNNL on the 21.14 tesla $\left({ }^{1} \mathrm{H}\right.$ resonance frequency of $\left.900 \mathrm{MHz}\right)$ instrument, we are examining sensitivity-enhancing techniques to study crystalline strontium samples and strontium-saturated clays and zeolites, in an attempt to characterize the local electromagnetic environment of strontium nuclei in these systems. Our recent collaboration using QCPMG approaches have led to a recent publication submission [(Bowers et al. 2005 (in submission)].

The use of the high-field NMR spectrometers has clearly been necessary for this work; without high-field NMR, our current understanding of soil phases would not be possible. The prospect of directly studying strontium salts in situ is compelling, even though it presents daunting technical challenges. The information gained could be crucial in understanding the scope of the contamination problem at Hanford and in better identifying appropriate strategies for improving cleanup.

Citation

Bowers GM, AS Lipton, and KT Mueller. 2005. "Solid-state ${ }^{87}$ Sr NMR Analyses of Salts and Natural Minerals at 21.14 T." Angewandte Chemie International Edition (paper submitted).

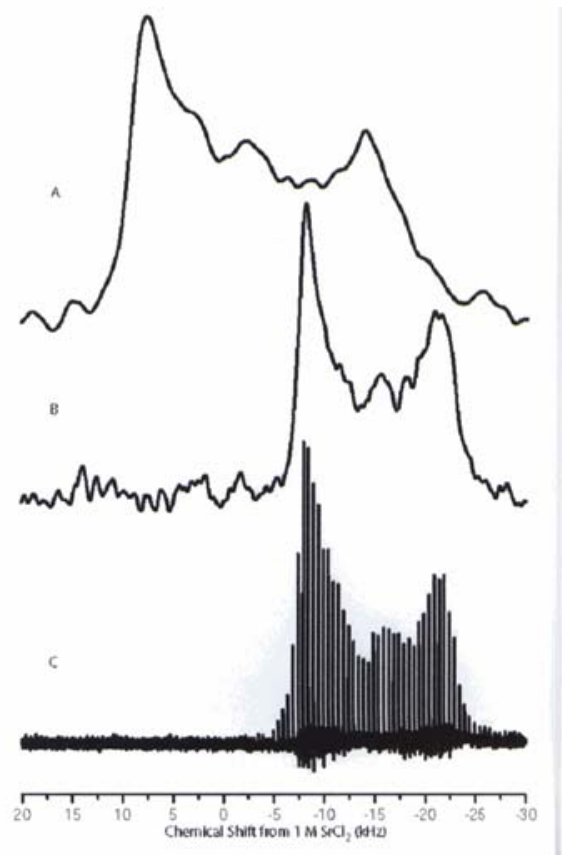

Figure 4-58. Strontium carbonate ${ }^{87} \mathrm{Sr}$ NMR spectra: (A) static echo at 11.74 tesla,

(B) static echo at 21.14 tesla,

(C) QCPMGat 21.14 tesla. 


\section{NMR Analysis of Methyl Groups in the 240kDa Arp2/3 Complex and Model Systems up to $560 \mathrm{kDa}$}

M Kreishman-Deitrick, ${ }^{(a)}$ C Egile, ${ }^{(b)}$ D W Hoyt, ${ }^{(c)}$ JJ Ford, ${ }^{(c)}$ R Li, ${ }^{(b)}$ and MK $\operatorname{Rosen}^{(\mathrm{a})}$

(a) University of Texas Southwestern Medical Center at Dallas, Dallas, Texas

(b)Harvard Medical School, Boston, Massachussets

(c) W.R. Wiley Environmental Molecular Sciences Laboratory, Richland, Washington

Signal-mediated rearrangements of the actin cytoskeleton are critical to many biological processes, including cell motility and morphology, bacterial and viral pathogenesis, and tumor metastasis. The $240 \mathrm{kDa}$ Arp $2 / 3$ actin nucleation complex has emerged as a key regulator of these rearrangements, and has consequently become an important target of structural studies. Recent work has revealed that regulation of the actin nucleating activity of Arp $2 / 3$ complex by the Wiskott-Aldrich Syndrome protein family proteins and filamentous $(\mathrm{F}-)$ actin is likely to involve a significant quaternary rearrangement in the complex. The nature of this conformational change and the binding modes of WASP proteins and $\mathrm{F}$-actin are not currently understood.

Biomolecular solution NMR is an extremely powerful tool with which to study the structure, conformational dynamics, and ligand interactions of macromolecules and assemblies. A significant obstacle facing NMR spectroscopy of large systems is the rapid deterioration of NMR signals due to fast transverse relaxation. While most of the work to date has focused on NMR signals from protein amide groups, sidechain methyl groups offer a useful alternative spectroscopic probe in large systems. Methyl groups have several advantages over amides in terms of their utility in NMR spectroscopy. Methyl protons are not exchangeable with solvent, whereas amide proton line widths are inherently broadened by solvent exchange. ${ }^{1} \mathrm{H}-{ }^{13} \mathrm{C}$ correlation spectra are generally disperse and well resolved, and methyl ${ }^{13} \mathrm{C}$ and ${ }^{1} \mathrm{H}$ lines are inherently narrower as a result of rapid rotation of the three protons about the methyl symmetry axis. The presence of three protons per methyl group provides higher sensitivity and efficient longitudinal relaxation through intramethyl dipolar interactions, resulting in short required $\mathrm{T} 1$ relaxation delays in NMR pulse sequences, even in systems highly deuterated at non-methyl positions. It has been shown that for ${ }^{1} \mathrm{H},{ }^{13} \mathrm{C}$ (methyl)/U-15 $\mathrm{N},{ }^{2} \mathrm{H}$-labeled samples of MBP $(42 \mathrm{kDa})$ and DHNA $(110 \mathrm{kDa})$, a simple ${ }^{1} \mathrm{H} /{ }^{13} \mathrm{C}-\mathrm{HSQC}$ spectrum of the methyl resonances is three-fold and -10-fold more sensitive, respectively, than a $1 \mathrm{H} / 15 \mathrm{~N}-\mathrm{TROSY}$ spectrum of the amides in the same sample. Methyl groups are also enriched in protein interiors and at protein- 
protein interfaces, and as with amide moieties, perturbation of methyl chemical shifts can be used to map ligand interactions.

Our time at EMSL has allowed us to establish the feasibility and scope of using methyl groups in NMR spectroscopy in molecules as large as $560 \mathrm{k}$ Da. To establish the general utility of methyl groups in large systems, we used a test sample consisting of a $16-\mathrm{kDa}$ protein suspended in a solution of 40 percent $\mathrm{v} / \mathrm{v}$ glycerol. By exploiting the steep temperature dependence of glycerol solution viscosity, we were able to simulate a range of rotational correlation times (60 to $330 \mathrm{~ns}$ ), which corresponds to molecular weight range of about 100 to 560 $\mathrm{kDa}$. The use of the high-field spectrometers available at EMSL allowed us to do a systematic, thorough analysis of ${ }^{1} \mathrm{H} /{ }^{13} \mathrm{C}-\mathrm{HSQC}$ spectra of this protein at a variety of temperatures and at two different fields (Figure 4-59). We find that signals from well-resolved methyl resonances can be detected in a 4.5-hour ${ }^{1} \mathrm{H} /{ }^{13} \mathrm{C}-\mathrm{HSQC}$ experiment on our $1.1 \mathrm{mM}$ model sample even at $\tau_{\mathrm{C}} \approx 330 \mathrm{~ns}$, and intense signals can easily be detected in a 100-hour spectrum of $\sim 87 \mu \mathrm{M}$ Arp2/3 complex. 'These results indicate that protonated methyl groups will be powerful tools for the study of large macromolecular systems by NMR, even at significantly lower concentrations. We also took advantage of the high-field instruments at EMSL to compare the above methyl spectra with ${ }^{1} \mathrm{H} /{ }^{15} \mathrm{~N}$ TROSY and CRINEPT-TROSY spectra on the same sample, and discovered that the methyl HSQC is in fact more sensitive than the amide experiments.

With the work we have done at EMSL, we have established sidechain methyl groups as extremely sensitive probes for NMR spectroscopy of large molecules. 'The acquired ${ }^{1} \mathrm{H} /{ }^{13} \mathrm{C}-\mathrm{HSQC}$ spectra of Arp2/3 complex will allow us to dissect the interactions of this assembly with its activators. 'These studies will provide detailed insight into the mechanism of activation of this important cellular machine.

\section{The following publication has resulted from this work at EMSL:}

Kreishman-Deitrick M, C Egile, D Hoyt, J Ford, R Li, and MK Rosen. 2003. "NMR Analysis of Methyl Groups in the 240kDa Arp2/3 Complex and Model Systems up to $560 \mathrm{kDa}$." Biochemistry 42(28):8579-8586.

\section{Progress Towards Structural Determination of a Complex Membrane Protein, Diacylglycerol Kinase}

CR Sanders ${ }^{(a)}$ and FD Sönnichsen ${ }^{(b)}$

(a) Vanderbilt University, Nashville, Tennesee

(b) Case Western Reserve University, Cleveland, Ohio

Prokaryotic diacylglycerol kinase (DAGK) is a homotrimeric integral membrane protein composed of $13-\mathrm{kDa}$ subunits, with each subunit having

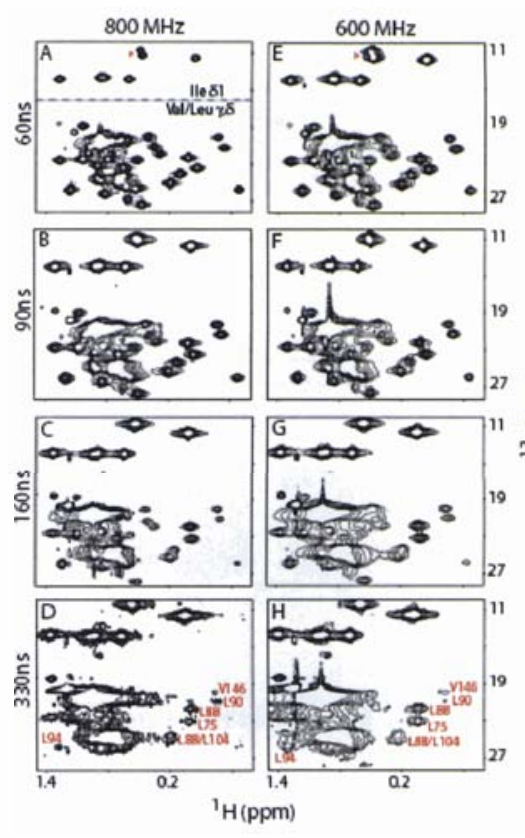

Figure 4-59. ${ }^{1} \mathrm{H} /{ }^{13} \mathrm{C}-\mathrm{HSQC}$ spectra of a $16 \mathrm{kDa}$ protein in 40 percent $v / v$ glycerol. Spectra were acquired at the fields indicated, and at the following temperatures: $25^{\circ} \mathrm{C}$ (panels $\mathrm{A}$ and E), $15^{\circ} \mathrm{C}(\mathrm{B}$ and $\mathrm{F}), 0^{\circ} \mathrm{C}(\mathrm{C}$ and $\mathrm{G})$ and $-10^{\circ} \mathrm{C}(\mathrm{D}$ and $\mathrm{H})$. Estimated $\tau_{\mathrm{C}}$ values for each temperature are shown to the left of each row. 
three transmembrane helices (Figure 4-60). As a membrane protein, DAGK represents a class of proteins, which is of enormous biological and biomedical importance, but which has proven largely refractory to structural determination by classical NMR and crystallographic methods. However, there has been recent and impressive progress in the area of membrane protein NMR, represented by the determination of monomeric beta barrel integral membrane porin structures (ca. $17 \mathrm{kDa}$ ) in detergent micelles by the Tamm/Bushweller, Wuthrich, and Kay labs. DAGK represents a target for NMR structural

\section{Diacylglycerol Kinase}

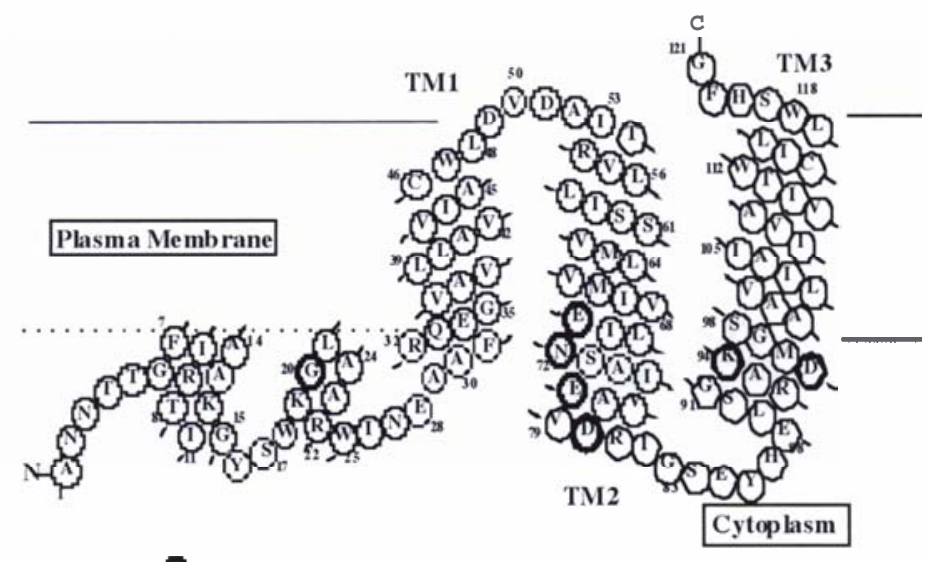

O Absolutely Conserved Site analysis which is at least one step beyond the porins in terms of difficulty because of its much larger size (40 $\mathrm{kDa}$ homotrimer) and because it is a largely helical protein. Determination of the structure of DAGK by solution NMR would be an important achievement for several reasons. If the structure of DAGK can be solved, then it should be possible to also tackle the important $\mathrm{G}$ protein-coupled receptors, which tend to have roughly the same molecular weight and number of transmembrane segments as the DAGK homotrimer. These proteins are the target of at least one-third of all known drugs. Prokaryotic DAGK is itself of considerable interest for several reasons. It plays a critical function on a metabolic pathway (the membrane-derived oligosaccharide cycle) which is not present in eukaryotes and which therefore may be a target for novel antimicrobial agents. DAGK is also of interest as a system for studying membrane protein folding and stability and for studying membrane biocatalysis. Indeed, we and our collaborators have carried out extensive structure-function studies of DAGK which has led to assignment of specific roles for many residues in protein stability and catalytic function (Figure 460). The Bowie lab at the University of California, Los Angeles has also been attempting to grow high-quality crystals of DAGK for about seven years. While crystals can be grown, they diffract to only 11 angstroms.

In collaboration with Dr. Sonnichsen, the Sanders lab has systematically optimized conditions under which high resolution NMR methods can be successfully applied to DAGK as seen by the data in Figure 4-61. We now have reliable methods for producing triple-labeledDAGK and for preparing samples of this enzyme in detergent micelles, where the DAGK trimer/detergent aggregate mass is ca. $100 \mathrm{kDa}$. DAGK concentrations of $\mathbf{3} \mathrm{mM}$ can be achieved and samples are completely stable for at least a week at $45^{\circ} \mathrm{C}$.

EMSL has been quite generous by providing us with blocks of $800-\mathrm{MHz}$ NMR spectrometer time over the past few years. Based upon TROSY-based threedimensional experiments conducted at EMSL using perdeuterated DAGK, we have completed assignment of about 90 percent of DAGK's 120 native backbone resonances (Figure 4-61). We have data that suggest that many of the remaining 10 percent of unassigned amide resonances are absent in the spectra because of a failure to completely back-exchange amide deuterons for protons following biosynthetic sample perdeuteration. We are now in the process of working out conditions for forcing such back-exchange so that the unassigned amide 
resonances can be viewed in otherwise fully perdeuterated samples. Moreover, we have also published two papers (Oxenoid et al. 2002 and 2004) that present a preliminary description of DAGK's secondary structure based on analysis of the ${ }^{15} \mathrm{~N}$ and ${ }^{13} \mathrm{C} \mathrm{NMR}$ chemical shifts measured for assigned residues as well as complete assignments. The instrument time provided by EMSL and the assistance of Dr. David Hoyt are acknowledged in those papers. It should also be noted Dr. Sanders acknowledged EMSL's support at the end of presentations on the DAGK NMR work at international conferences during the past year.

With assignments nearing completion, the shift of the DAGK NMR project is towards the acquisition of restraints for structural determination (nuclear Overhauser effects and residual dipolar couplings). While the Sanders lab has moved to Vanderbilt, the collaboration between his lab and that of Dr. Sonnichsen continues. Also, while there is an in-house $800-\mathrm{MHz}$ NMR at Vanderbilt, we will continue to request time at EMSL, particularly for the $900-\mathrm{MHz}$ instrument. Experiments to measure NOEs and dipolar couplings are likely to be extremely demanding because of the relatively wide line widths yielded by DAGK (even in TROS Y spectra) and resulting sensitivity problems-such measurements should benefit significantly from access to the $900-\mathrm{MHz}$ instrumentation.

\section{The following publications have been generated as a result of this research:}

Ashish A, F Abildgaard,JH Bushweller, and LK Tamm. 2001. "Structure of Outer Membrane Protein A Transmembrane Domain by NMR Spectroscopy." Nature Structural Biology 8:334-338.

Fernandez C, K Adeishvili, and KWuthrich. 2001. "TROSY with the Outer Membrane Protein OmpX in Dihexanoyl PhosphatidylcholineMicelles." Proceedings of the National Academy of Sciences 98:2358-2363.

Hwang PM, W Choy, EI Lo, L Chen, JD Formay-Kay, CRH Raetz, GG Prive, RE Bishop, and LE Kay. 2002. "Solution Structure and Dynamics of the Outer Membrane Protein PagP by NMR." Proceedings of the National Academy of Sciences 99:13560-13565.

Kennedy EP. 1996. "Membrane-derived Oligosaccharides of E. Coli. In "E. Coli and S. Typhimurium." Cellularand Molecular Biology I:1064-1071, F. C. Neidhardt, Ed., ASM Press, Washington.

Lau FW, X Chen, and JU Bowie. 1999. "Active Sites of E. Coli DAGK are Shared Between Subunits." Biochemistry 38:5521-5527.

Nagy JK, WL Lonzer, and CR Sanders. 2001. "Kinetic Study of Folding and Misfolding of Diacylglycerol Kinase in Model Membranes." Biochemistry 40:8971-8980.

Oxenoid K, HK Kim, J Jacob, FD Sonnichsen, and CR Sanders. 2004. "NMR Assignments for a Helical $40 \mathrm{kDa}$ Membrane Protein in Micelles." Journal of the American Chemical Society 126:5048-5049.

Oxenoid K, FD Soennichsen, and CR Sanders. 2001. "Conformationally-specific Misfolding of an Integral Membrane Protein." Biochemistry 40:5111-5118.

Sanders CD and K Oxenoid. 2000. "Customizing Model Membrane and Samples for NMR Spectroscopic Studies of Complex Membrane Proteins." Biochimica et Biophysica Acta 1508:129-145.

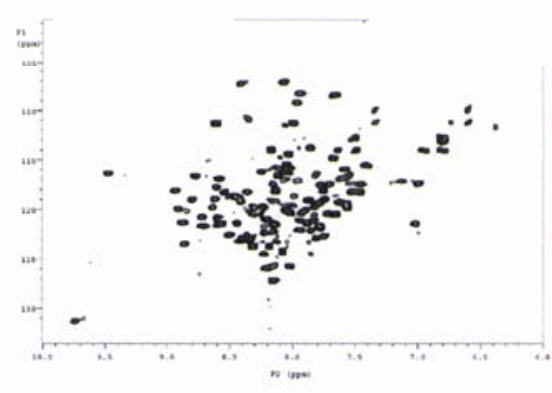

Figure 4-61. 800-MHz TROSY Spectrum of Triple Labeled DAGK in DPC Micelles at 45 degrees acquired at EMSL. 80 percent of DAGK's backbone amide resonances have now been assigned (Vinogradova et al. 1997). (Downfield Trp indole NH peaks are not shown.) 
is composed of a network of dihydrogen bonds formed between the protic $\mathrm{H}^{+}$ attached to nitrogen and hydridic $\mathrm{H}^{-}$attached to boron.

Preliminary results showed the rates of hydrogen release from the bulk phase solid ammonia borane follows an apparent nucleation and growth kinetic model. However, little is known about the nucleation events and the role of the intermolecular dihydrogen bonding in the formation of molecular hydrogen. Solid-state nuclear magnetic resonance (SSNMR) ${ }^{11} \mathrm{~B}\left\{{ }^{1} \mathrm{H}\right\}$ spectra of these reactions taken at $300-\mathrm{MHz}{ }^{1} \mathrm{H}$ frequency aided in determining reaction mechanisms; however, some products remain unidentified due to spectral overlap. NMR experiments run using higher fields $(500 \mathrm{M} \mathrm{Hz}$ and $800 \mathrm{MHz}$ ) enhanced resolution and reduced the quadrupolar coupling, which simplified the distinction between quadrupolar coupling and multiple species reaction products formed as a result of hydrogen release (Figures 4-62 and 4-63). In some cases, $500 \mathrm{MHz}$ was adequate to identify products, but $800 \mathrm{M} \mathrm{Hz}$ was needed to clear ambiguities, as shown in Figure 4-63. Collecting high-field NMR data on some representativesamples can greatly aid the interpretation of lower field data.

In addition to the ${ }^{11} \mathrm{~B}$ SSNMR experiments, ${ }^{1} \mathrm{H}$ SSNMR spectra were also attained at $800 \mathrm{MHz}$. Each of the starting materials and reaction products were studied. As expected, chemical shift differences were observed. These observations will be further quantified using heteronuclearcorrelation to correlate the ${ }^{1} \mathrm{H}$ and ${ }^{11} \mathrm{~B}$ resonances to provide further product information. ${ }^{1} \mathrm{H}$ experiments are also being used to investigate scaffolding properties. We found that scaffolding the $\mathrm{NH}_{3} \mathrm{BH}_{3}$ into mesoporus silica reduces the temperature for hydrogen release. The chemistry and thermodynamics behind this observation are not understood; however, the extreme narrowing in the proton NMR would suggest either an increased ordering or a more liquid-like behavior in the scaffold (Figure 4-64). Further experiments such as rotational echo double resonance for the direct investigation of atomic distances are necessary to propose a mechanism, but the initial insight provides evidence of a fundamentally different organization within the scaffold.

\section{A Radius of Curvature Analysis of a 16- Base-Pair DNA Oligomer Provides New Insight into Global DNA Curvature}

K McAteer, ${ }^{(a)}$ A Aceves-Gaona, ${ }^{\left({ }^{a}\right)}$ R Michalczyk, ${ }^{(b)}$ G W Buchko, ${ }^{(c)}$ NG Isern, ${ }^{(c)}$ LA Silks, ${ }^{(b)}$ JH Miller, ${ }^{(a)}$ and MA Kennedy ${ }^{(c)}$

(a) Washington State University-Tri-Cities, Richland, Washington

(b)Los Alamos National Laboratory, Los Alamos, New Mexico

(c) Pacific Northwest National Laboratory, Richland, Washington

In-phase ligated DNA containing TnAn segments fail to exhibit the retarded polyacrylamide gel electrophoresis migration observed for in-phase ligated

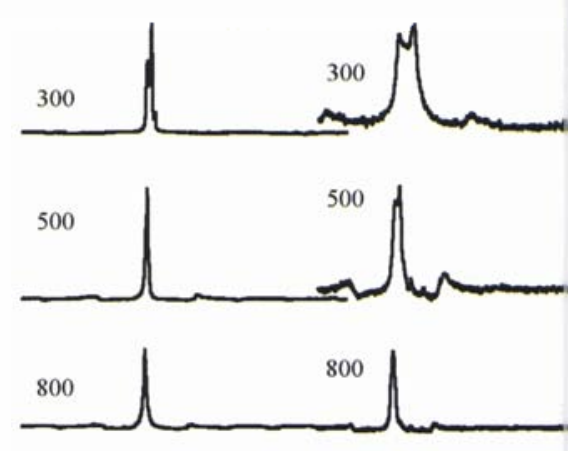

Figure 4-63. SSNMR ${ }^{11} \mathrm{~B}\left\{{ }^{1} \mathrm{H}\right\}$ spectra as a function of field, spinning at $10 \mathrm{kHz}$. The starting material (left) and the final reaction products (after heating at $170^{\circ} \mathrm{C}$-right) are shown at all three fields. For some compounds, such as $\mathrm{NH}_{3} \mathrm{BH}_{3}$ (left), $500 \mathrm{MHz}$ provides maximum narrowing. For some of the products (right), $800 \mathrm{MHz}$ is needed to sufficiently narrow the peaks. Investigating at all three fields provides important information about the coupling constant, the symmetry and the products.

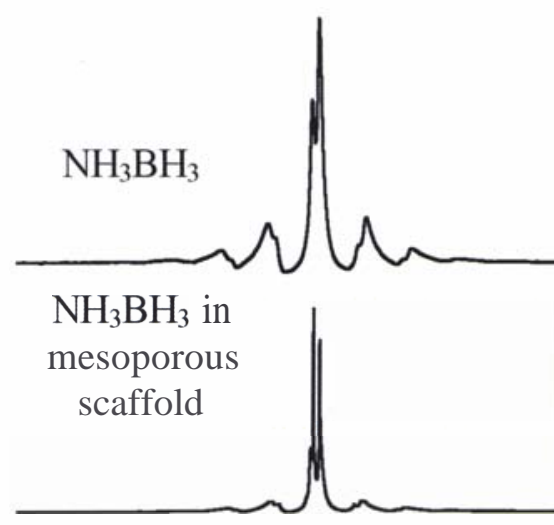

Figure 4-64. ${ }^{1} \mathrm{H}$ SSNMR (spinning speed $15 \mathrm{kHz}$ ) reveals a distinct narrowing of the two resonances as a function of scaffolding $\mathrm{NH}_{3} \mathrm{BH}_{3}$ into mesoporous silica. 


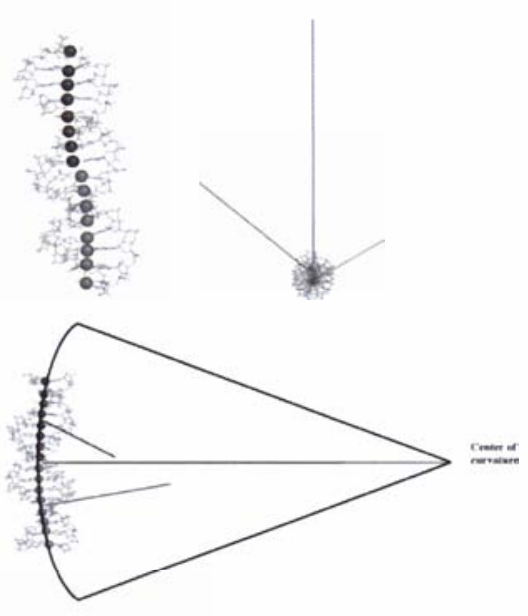

Figure 4-65. Global curvature of the average RDC structure (top left) of the $\mathrm{T}_{3} \mathrm{~A}_{3} 16 \mathrm{mer}$ analyzed by fitting a circle to the helical axis reference points (blue/pink balls). (b) $R_{C}$ for the whole molecule (red), and for the upper (blue) and lower (pink) halves, looking parallel to the helix axis (top right). (c) $R_{c}$ for the whole molecule (red) and for the upper (blue) and lower (pink) halves, looking perpendicular to the helix axis (bottom).
An'Tn segments, a behavior thought to be correlated with macroscopic DNA curvature. The lack of macroscopic curvature in ligated TnAn segments is thought to be due to cancellation of bending in regions flanking the TpA steps. To address this issue, solution-state NMR, including residual dipolar coupling restraints (Figure 4-65), was used to determine a high-resolution structure of [d(CGAGGTTTAAACCTCG $)_{2}$, a DNA oligomer containing a $\mathrm{T}_{3} \mathrm{~A}_{3}$ tract. 'The overall magnitude and direction of bending, including the regions flanking the central TpA step, was measured using a radius of curvature, Rc, analysis. The Rc for the overall molecule indicated a small magnitude of global bending ( $\mathrm{Rc}=138 \pm 23 \mathrm{~nm}$ ) towards the major groove, whereas the Rc for the two halves $(72 \pm 33 \mathrm{~nm}$ and $69 \pm 14 \mathrm{~nm}$ ) indicated greater localized bending into the minor groove. The direction of bending in the regions flanking the $\mathrm{TpA}$ step is in partial opposition $\left(109^{\circ}\right)$, contributing to cancellation of bending. The cancellation of bending did not correlate with a pattern of roll values at the $\mathrm{TpA}$ step, or at the 5-foot and 3-foot junctions, of the $\mathrm{T}_{3} \mathrm{~A}_{3}$ segment, suggesting a simple junction/roll model is insufficient to predict cancellation of DNA bending in all TnAn junction sequence contexts. Importantly, Rc analysis of structures refined without residual dipolar coupling restraints lacked the precision and accuracy needed to reliably measure bending (McAteer et al. 2004).

Although residual dipolar coupling restraints have been shown to be essential for accurate NMR-based DNA structure determination, improvements in data collection have also been necessary for obtaining reliable initial starting structures for calculations with residual dipolar coupling restraints. The collaboration between PNNL and Los Alamos National Laboratory has led to new and improved pulse sequences for measuring proton-proton coupling constants that are necessary for analyzing the sugar conformation(s) in DNA (Yang et al. 1997 and 2000).

\section{Citations}

McAteer K, A Aceves-Gaona, R Michalczyk, GW Buchko, NG Isern, LA Silks, JH Miller, and MA Kennedy. 2004. "compensating Bends in a 16 Base-Pair DNA Oligomer Containing a TnAn Segment: A NMR Study of Global DNA Curvature." Biopolymers 75:497-511.

Yang J, KMcAteer, LA Silks, R Wu, NG Isern, CJ Unkefer, and MA Kennedy. 2000. "A Comprehensive Approach for Accurate Measurement of Proton-Proton Coupling Constants in the Sugar Ring of DNA." Journal of Magnetic Resonance 146:260-276.

Yang J, LA Silks, R Wu, NG Isern, CJ Unkefer, and MA Kennedy. 1997. "Improvements for Measuring ${ }^{1} \mathrm{H}^{-1} \mathrm{H}$ Coupling Constants in DNA via New StripeCOSY and Superstripe-COSY Pulse Sequences Combined with a Novel Strategy of Selective Deuteration." Journal of Magnetic Resonance 129:212-218. 


\section{Structure of Telomerase and Telomeric Proteins}

T Leeper, (a) SL Reichow, (a) Y Chen, (a) JK Fender, ${ }^{(a)}$ KS Godin, (a) and G Varani (a)

(a) University of Washington Seattle, Washington

Telomerase is the ribonucleoprotein enzyme responsible in most eukaryotes for the replication of the chromosome termini (telomeres). It also plays a critical role in cellular division and cancer: it is activated in the germ line and in the great majority of cancer cells. In humans, it is composed of a 400-nucleotide RNA and several proteins that associate with it and carry out the enzymatic activity and promote its cellular localization and assembly. We are studying the structure of two critical domains of telomerase RNA responsible for RNA biogenesis and for recruitment of the catalytic activity to the holoenzyme, respectively. Four proteins of unknown structure bind to human telomerase RNA: in doing so, they stabilize the RNA in the cell and direct its processing and nuclear localization. We aim to determine the structures of two such proteins (called NoplO and Garl) and of the RNA domains they associate with. NMR studies conducted in Seattle and at PNNL have provided us with very-high-quality data for all three proteins and for a key domain of human and lower eukaryotic telomerase RNAs. NMR experiments conducted at PNNL have contributed significantly to our progress towards determining the structures of critical domains of human telomerase RNA and its associated proteins.

Structure of telomerase bindingproteins - NoplO, plays an essential function in the biogenesis of telomerase and of the ribosome. NMR spectra of very high quality collected in part at PNNL have allowed us to determine the structure of the archeal and yeast proteins (Figure 4-66). The archeal protein forms a zinc-knuckle packed against a $\mathrm{C}$-terminal helix. The yeast and human proteins have lost the zinc-binding domain, but Nop10 retains a similar overall fold, though with considerably increased flexibility. Data collected at PNNL are also allowing structure determination of the archeal and yeast Gar1 proteins. Because of high levels of sequence homology, the structure of the human protein will be reliably modeled once these studies are completed. Although Garl had been proposed to be related to Sm proteins, a family of RNAbinding protein that form ring-like structures on U-rich RNAs, in actuality this protein forms an all- $\boldsymbol{\beta}$ structure of with a different fold.

The activation domain of human telomerase - Human and mouse telomerase RNAs retain a domain called CR4-CR5 capable of reconstituting telomerase activity when combined with the template domain. It is a key functional element of vertebrate telomerases that, we hypothesize, is conserved in all eukaryotes, including ciliates. 'The NMR work on the human CR4/CR5 domain conducted at PNNL has progressed as shown in Figure 4-67 (Leeper et al. 2005, in press)
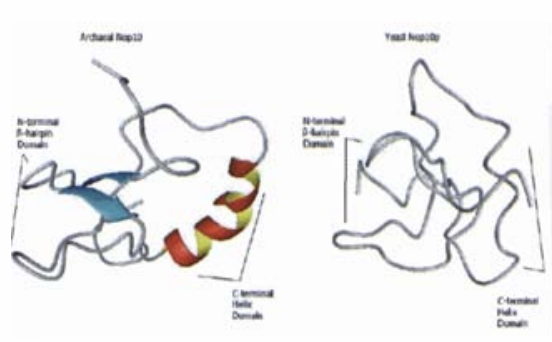

Figure 4-66. NMR structures of archea (left) and yeast (right) NoplO proteins determined in part using data collected at PNNL

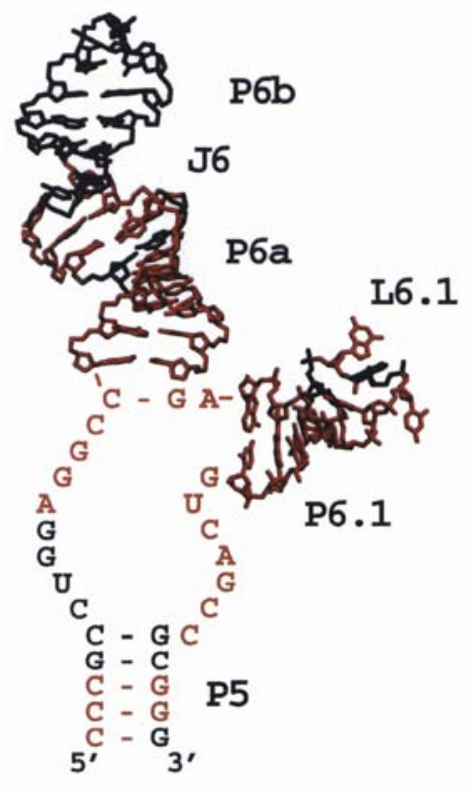

Figure 4-67. Current progress in the structure determination of the domain of human telomerase RNA responsible for activation of the enzymatic activity 
electrochemical potential of protons drives the synthesis of ATP via a chemiosmotic circuit. "The cyt $b c$ complexes are broken into two classes: $b c_{1}$ and $b_{6} f$ types, although forms intermediate between the $b_{c_{1}}$ and $b_{6} f$ complexes have been found in some bacteria. Both cyt $b_{c_{1}}$ and $b_{6} f$ complexes contain four redox-active, metal centers: one heme c, one Rieske-type $\mathrm{Fe}_{2} \mathrm{~S}_{2}$ center, and two b-type hemes. 'These metal centers are found in three proteins: heme c in cyt $c_{1}$ (or cyt $f$ in $b_{6} f$ ); the Rieske $\mathrm{Fe}_{2} \mathrm{~S}_{2}$-protein (ISP); and the $b$ hemes in a single cyt $\boldsymbol{b}$ (or cyt $b_{6}$ in $b_{6} f$ ).

'The working model for most research in this area is the Q-cycle, where two protons are pumped across the energetic membrane for each electron transferred from quinol to a high potential acceptor. The electron transfer is a bifurcated process, as illustrated in Figure 4-68. One electron from the hydroquinone (or quinol) in site $\mathbf{Q}$ (labeled S Q in Figure 4-68) is transferred to the Rieske $\mathrm{Fe}_{2} \mathrm{~S}_{2}$ center while the other is forced to flow through a chain of two cyt $b$ hemes to reduce a quinone in site $\mathrm{Q}_{i}$ (labeled $\mathrm{SQ}_{i}$ in Figure 4-68). Both protons from site $\mathbf{Q}$ are ultimately released on the $\mathrm{p}$-side of the membrane while the quinone at the Q site picks up two protons from the n-side of the membrane. 'Thus, since the bifurcation of the electron flow at the $\mathbf{Q}$ site allows only one quinol electron to pass to the high-potential electron acceptor (i.e., cyt $\mathrm{c}$ in mitochondria, plastocyanin or cyt $c_{6}$ in chloroplasts), it doubles the expected number of protons pumped per electron and accounts for the high efficiency of energy transduction by the bc complexes.

The recent publication of high-resolution x-ray structures of mitochondrial cyt $b c_{1}$ complexes has greatly accelerated progress in understanding the function of these enzymes. One notable feature of the crystal structures is a variation in the position of the "head" (or hydrophilic extension) of the ISP. The presence of two distinct ISP conformationsled to the "domain movement ${ }^{\mathrm{n}}$ hypothesis where the ISP pivots back and forth to "gate ${ }^{\mathrm{n}}$ electron transfer, forcing the two electrons on the quinol to be transferred through different pathways. In one conformation, quinol is bound at the $\mathrm{Q}_{6}$ site and transfers one electron to the $\mathrm{Fe}_{2} \mathrm{~S}_{2}$ center. Further transfer of this electron is prevented because the ISP in this conformation is distant $(\sim 31 \AA)$ from cyt $c_{1}$. Thus, the remaining semiquinone electron is transferred to the low potential cyt $b_{L}$ then to cyt $b_{H}$ and finally to a quinone or semiquinone bound at the Qsite. Only after full oxidation of quinol does the ISP pivot and make close contact with cyt $c_{1}$, allowing electron transfer to mobile cyt c. In collaboration with EMSL, we have confirmed such motion EPR measurements on partially oriented samples of the cyt $b_{6} f$ complex. A further significant advance was the discovery that found that that certain metal ions inhibit the cyt $b_{6} f$ complex by binding to cyt $f$ and interfering with ISP domain movements (Figure 4-69).

In research supported by the National Science Foundation and DOE, we have shown that, regardless of the in vivo energy status of the plant, electron transfer through the cyt $b_{6} f$ complex appears tightly coupled to the pumping of protons. Our current work focuses on determining why this coupling is so tight. One of the key obstacles in answering this question has been the lack of knowledge about how substrate interacts with the active site. We have made

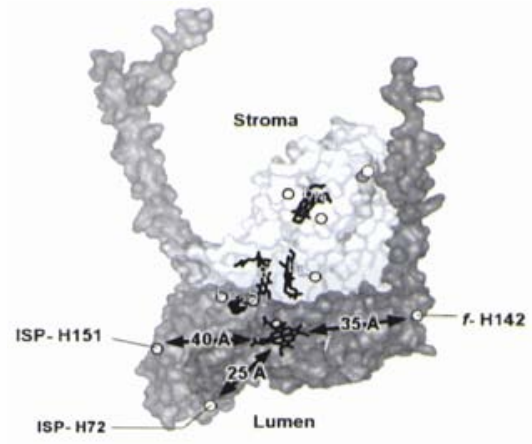

Figure 4-69. A model of the complete cyt $b_{6} f$ complex based on X-ray structures of the cyt $f$ and ISP domains and on homology with the cyt $b c_{1}$ structures (See Figure 4-70), which shows the location of bound $\mathrm{Cu}^{2+}$ determined by pulsed $E P R$

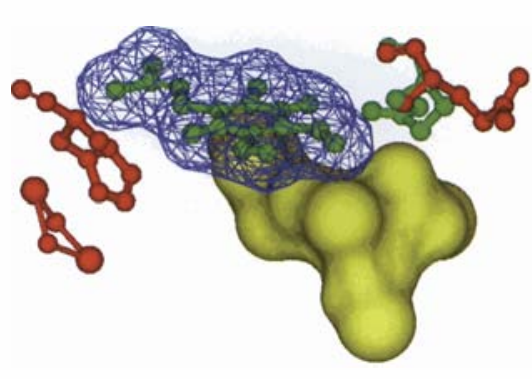

Figure 4-70. Characterization of the partial reactions observed in the presence of certain inhibitors suggests a structure for the substrate bound to the cyt $b c_{1}$ complex. Shown is a hypothetical structure of ubiquinol (blue mesh) co-bound to residues of the substrate binding site (red/green) in the presence of the inhibitor mucidin (yellow). 
two significant contributions towards defining the substrate-binding site, again in collaboration with Michael K. Bowman at EMSL. First, we have shown that two quinone analogs can bind simultaneously to the site. Secondly, we have been able to define the structural bounds of substrate binding by probing the partial reactions of the enzyme in the presence of a series of different inhibitors (Figure 4-70).

\section{The followingpublications have resulted from our work using the EPR capability at EMSL's High-Field Magnetic Resonance Facility:}

Bowman MK, EA Berry, AG Roberts, and D M Kramer. 2004. "Orientation of the g-Factor Axes of the Rieske Subunit in Cytochrome bcl Complex." Biochemistry 430436.

CooleyJW, AG Roberts, MK Bowman, D M Kramer, and F Daldal. 2004. "The Raised Midpoint Potential of the [2Fe2S] Cluster of Cytochrome bcl is Mediated by Both the $\mathbf{Q}$ Site Occupants and the Head Domain Position of the Fe-S Protein Subunit." Biochemisty 43(8):2217-2227.

Muller FL, AG Roberts, MK Bowman, and D M Kramer. 2003. "The Architecture of the Q Site of Cytochrome bcl Complex Probed by Superoxide Production." Biochemistry 42(21):6493-6499.

Muller, F, AR Crofts, and D M Kramer. 2002. "Multiple Q-cycle Bypass Reactions at the Q-site of the Cytochrome bcl Complex." Biochemistry 41:7866-7874.

Rao B K S, A M Tyryshkin, AG Roberts, MK Bowman, and D M Kramer. 2000. "Inhibitory Copper Binding Site on the Spinach Cytochrome b(6)f Complex: Implications for Q(0) Site Catalysis." Biochemisty 39(12):3285-3296.

Rao SB, A M Tyryshkin, MK Bowman, and D M Kramer. 1999. "Bound $\mathrm{Cu}^{2}+$ as a Structural Probe of the Cytochrome b6f Complex, Photosynthesis: Mechanisms and Effects." Kluwer Academic Publishers, The Netherlands, Netherlands.

Roberts AG, MK Bowman, and D M Kramer. 2004. "'The Inhibitor DBMIB Provides Insight into the Functional Architecture of the $Q_{6}$ Site in the Cytochrome b6f Complex." Biochemistry 43(24):7707-7716.

Roberts AG, MK Bowman, and DM Kramer. 2002. "Certain Metal Ions are Inhibitors of Cytochrome b (6) f Complex 'Rieske' Iron-Sulfur Protein Domain Movements." Biochemisty 41(12):4070-4079.

Roberts, A, and DM Kramer. 2001. "Inhibitor 'Double-occupancy' in the Qo Pocket of the Chloroplast Cytochrome $b_{6} f$ Complex." Biochemistry 40(45):13407-13412.

Zhang H, AN Primak, JL Cape, MK Bowman, D M Kramer, and W A Cramer. 2004. "Characterization of the High Spin Heme $\mathrm{x}$ in the Cytochrome b6f Complex of Oxygenic Photosynthesis." Biochemistry 43(51):16329-16336. 


\title{
Polyphosphate Amendments for In-Situ Immobilization of Uranium Plumes
}

\author{
DM Wellman, (a) JP Icenhower, ${ }^{(a)}$ EM Pierce, (a) BK McNamara, (a) \\ SD Burton, ${ }^{(b)}$ KN Geiszler, ${ }^{(a)}$ and SR Baum ${ }^{(a)}$ \\ (a) Pacific Northwest National Laboratory, Richland, Washington \\ (b)W.R. Wiley Environmental Molecular Sciences Laboratory, Richland, \\ Washington
}

\begin{abstract}
Operations related to nuclear energy and weapons production have resulted in global uranium contamination of surface and subsurface geologic media (Abdelouas et al. 1999). Within the D OE complex, uranium has been recognized as one of the two most frequently occurring radionuclides in groundwater and the most frequently occurring radionuclide in soils/sediments (Riley et al. 1992). The potential long-term risk to underlying groundwater and associated environmental hazards has caused remediation of uraniumcontaminated soils to be a high priority for $\mathrm{DOE}$ facilities. In addition to contaminated soils, performance assessment calculations associated with disposal of low-activity waste in a shallow burial ground have shown that groundwater transport presents the greatest potential risk of radionuclide exposure (Mann et al. 2001).

Typical of the arid western United States, Hanford Site sediments are dominated by sand and the groundwater has a $\mathrm{pH}$ of -8.5 with a dissolved $\left[\mathrm{CO}_{3}{ }^{2}-\right]$ of $-67.5 \mathrm{mg} / \mathrm{L}$ (Kaplan et al. 1995). Under these conditions uranium, as uranyl $\mathrm{UO}_{2}{ }^{2+}$, is predicted to form anionic carbonate complexes, $\mathrm{UO}_{2}\left(\mathrm{CO}_{3}\right)_{2}{ }^{2-}, \mathrm{UO}_{2}\left(\mathrm{CO}_{3}\right)_{3}{ }^{4-}, \mathrm{UO}_{2}(\mathrm{OH})_{2}$, and $\mathrm{UO}_{2}(\mathrm{OH})_{3}{ }^{1-}$ (Kaplan et al. 1995). Sorption of these anionic complexes is often assumed to be zero, particularly in sand.
\end{abstract}

More discussion describing the problems associated with uranium plume control and approaches for solving it are available elsewhere, but the following research focuses on the study of phosphate amendment strategy and the resultant ${ }^{31} \mathrm{P}$ NMR analysis for studying hydrolyzation kinetics.

One phosphate amendment strategy employs water-soluble phosphate compounds that could be injected into a uranium plume from strategically placed wells. For example, Lee et al. 1995 proposed using tribasic sodium phosphate $\left[\mathrm{Na}_{3}\left(\mathrm{PO}_{4}\right) \cdot n \mathrm{H}_{2} \mathrm{O}\right.$, or TSP] as a chemical stabilizer for uranium and radiostrontium. However, even in relatively dilute groundwater solutions, there were enough dissolved cations to react and form Al-, $\mathrm{Fe}-, \mathrm{Ca}-$, and $\mathrm{Na}-$ phosphates. Nash, Jensen, and coworkers at Argonne National Laboratory (Jensen et al. 1996; Nash et al. 1999) alleviated this shortcoming by proposing injection of a natural water-soluble organophosphate substance, phytic acid, into contaminated groundwater. The key advantage of this method is 
that the hydrolyzation kinetics of the molecule is slow, such that release of orthophosphate is delayed, allowing the injected, amending solution to disperse and mix throughout the target plume. One of the effects of injecting phytic acid solutions into realistic groundwater compositions is that a solid, calcium phytate precipitates and acts as a strong sorbent for uranium, even in solutions with high-ionic strength (Jensen et al. 1996; Nash et al. 1999). However, as demonstrated below, application of this amendment in saturated column experiments resulted in a $>30$ percent reduction in hydraulic conductivity. Altering the hydraulic conductivity of the subsurface by such a large magnitude will have a significant effect on subsequently injected amendment solutions, the targeted groundwater plume, or both, by deflecting flow from the natural path.

The purpose of this work was to evaluate long-chain condensed polyphosphates as a viable "time-released" source of phosphate for the in-situ immobilization of subsurface uranium plumes. Hydraulically saturated and unsaturated column tests demonstrate the ability of polyphosphate compounds to control the precipitation kinetics of insoluble phosphate minerals and optimize conditions for controlled application of phosphate amendments for subsurface remediation. X-ray microfocus tomography, in conjunction with column tests, illustrates the longterm effects of phosphate mineralization on hydraulic conductivity. ${ }^{31} \mathrm{P}$ NMR has been used to quantify the effect of sedimentary and aqueous components on the in-situ hydrolysis kinetics of condensed polyphosphates. Single-pass flow-through tests have been conducted to evaluate the longevity of precipitated uraniumphosphate minerals and quantify the effects of aqueous organic material on the dissolution kinetics of autunite minerals, $\mathrm{X}_{1-2}\left[\left(\mathrm{UO}_{2}\right)\left(\mathrm{PO}_{4}\right)\right]_{2} \cdot \mathrm{nH}_{2} \mathrm{O}$, under environmentally relevant conditions.

'Ihe portion of analysis being performed at EMSL is the study to follow hydrolyzation kinetics determined by ${ }^{31} \mathrm{P}$ NMR. The samples are being analyzed using the $300-\mathrm{MHz}$ NMR spectrometer to quantify the degradation of long-chain polyphosphates and the formation of orthophophate. Efforts to quantify specific degradation catalysts within natural groundwater were undertaken by conducting controlled heterogeneous batch tests between the polyphosphate solutions and pure sedimentary components (i.e. $\mathrm{Al}(\mathrm{OH})_{3}$, $\mathrm{CaOH}, \mathrm{CaCl}_{2}, \mathrm{Fe}(\mathrm{OH})_{3}, \mathrm{FeCl}_{3}$, quartz sand, and hydroxylapatite) and in dilute aqueous batch tests (i.e., $\mathrm{Ca}^{2+}, \mathrm{Mg}^{2+}, \mathrm{Na}^{+}, \mathrm{Al}^{3+}$ and $\mathrm{Fe}^{3+}$ ). The $\mathrm{pH}$ was controlled using a carbonate buffer to maintain the solution $\mathrm{pH}$ in a range reasonably near that of Hanford Site groundwater, $\mathrm{pH}$-8.5. Samples are filtered using $0.45 \mu \mathrm{m}$ syringe filters at predetermined intervals of one week for four weeks. ${ }^{31} \mathrm{P}$ NMR results show an increase in degradation of all three condensed phosphates to pyrophosphate, and monophosphate was observed to follow $\mathrm{Fe}(\mathrm{OH})_{3}>\mathrm{FeCl}_{3}>\mathrm{Al}(\mathrm{OH})_{3}>\mathrm{CaOH}>$ hydroxylapatite $>\mathrm{CaCl}_{2}>$ quartz sand for solid phase and concentrated materials. Preliminary results of dilute aqueous phase interactions indicate cation concentrations present in groundwater induce minimal catalysis of condensed poyphosphates degradation at $\mathbf{2 3}^{\circ} \mathrm{C}$. This suggests degradation of condensed polyphosphates is primarily influenced by heterogeneous surfaces. Use of NMR spectroscopy has been 
successfully applied to this in-situ engineering problem by providing new data for solving chemistry issues applicable to the sequestration of uranium in ground soil.

\section{Citations}

Abdelouas A, W Lutze, and HE Nuttall. 1999. "Uranium Contamination in the Subsurface: Characterization and Remediation." In: Uranium: Mineralogy, Geochemistry and the Environment. PC Burns and RJ Finch, Eds, pp. 679. Mineralogical Society of America, Washington, D.C.

Jensen MP, K Nash, J W Morse, EH Appelman, and M A Schmidt. 1996. "Immobilization of Actinides in Geomedia by Phosphate Precipitation." ACS Symposium Series \#651, p. 272-285.

Kaplan DI, RJ Serne, and M G Piepho. 1995. Geochemical Factors Affecting Radionuclide Transport through Near and Far Fields at a Low-Level Waste Disposal Site. Pacific Northwest Laboratory, Richland, Washington.

Lee SY, C W Francis, M E Timpson, and M P Elless. 1995. Radionuclide Containment in Soil by Phosphate Treatment. Oak Ridge National Laboratory, Oak Ridge, Tennessee.

Mann FM, RJ Puigh II, SH Finfrock, EJ Freeman, R Khaleel, D H Bacon, M P Bergeron, BP McGrail, and SK Wurstner. 2001. Hanford Immobilized LowActivity Waste Performance Assessment: 2001 Version. Pacific Northwest National Laboratory, Richland, Washington.

Nash K, LR Morss, M P Jensen, E H Appelman, M A Schmidt, S Friedrich, M Redko, and JJ Hines. 1999. Water-Soluble Organophosphorous Reagents for Mineralization of Heavy Metals. Argonne National Laboratory, Argonne, Illinois.

Riley RG, JM Zachara, and FJ Wobber. 1992. Chemical Contaminant on DOE Lands and Selection of Contaminant Mixtures for Subsurface Science Research. U.S. Department of Energy, Office of Energy Research, Washington, D. C. 
4.1.4

\section{High-Performance Mass Spectrometry Facility}

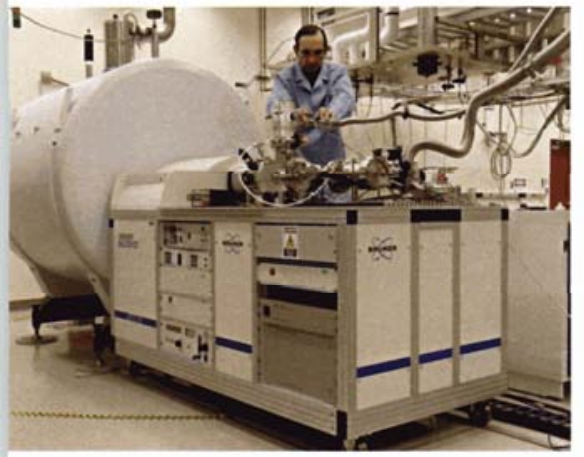

EMSL's new cutting-edge 12tesla FTICR mass spectrometer, located in the High-Performance Mass Spectrometry Facility.
The High-Performance Mass Spectrometry Facility provides EMSL users and researchers with state-of-the-art mass spectrometry and separations instrumentation that has been refined for leading-edge analysis of biological problems, with a primary emphasis on proteomics.

\section{Representative Research Activities}

Current research activities in the High-Performance Mass Spectrometry Facility include proteomic analyses of whole cell lysates, analyses of organic macromolecules and protein complexes, quantification using isotopically labeled growth media, targeted proteomics analyses of subcellular fractions, and nucleic acid analysis of RNA and DNA oligomers. More than a dozen microbial systems are currently studied in the facility by researchers from throughout the country. In addition, there are several ongoing projects in higher order systems (including mammalian systems) that are investigating a broad range of biological questions from cancer screening to infectious diseases to fundamental questions of post-translational modifications and protein-protein interactions.

\section{Research Capabilities}

- Five FTICR mass spectrometers, 3.5, 7, 9.4, 11.5 and 12 tesla, with electrospray ionization sources

- Custom high-performance liquid chromatography systems

- Agilent capillary high-performance liquid chromatography system

- Sciex QSTARR quadrupole time-of-flight mass spectrometer

- Five Finnigan LCQion trap spectrometers

- Finnigan TSQ7000 triple quadrupole spectrometer

- Ultrahigh-pressure liquid chromatographs.

\section{Recent Upgrades}

\section{Bruker Daltonics APEX 111 Data Station}

This new data station, installed on the 11.5-tesla FTICR spectrometer, permits automated operation of the spectrometer with custom high-performance liquid chromatography platforms.

\section{2-tesla Magnet}

The acquisition and installation of this 12-tesla, 110 -mm-bore actively shielded magnet was completed in Fall 2004 and replaces the facility's 3.5-tesla FTICR 
spectrometer. During the coming year, the vacuum system for the magnet will be installed and set up for analysis of intact proteins by electron capture dissociation, providing a top-down analysis capability that will help researchers understand the role, function, structure, and changing nature of complex cellular proteins. This top-down analysis will complement the bottom-up analysis that the facility currently offers, and will provide a more complete proteomics analysis capability.

\section{Future Direction}

'Ihe High-Performance Mass Spectrometry Facility will maintain a position at the forefront of mass spectrometry, using a systems biology approach to further the understanding of biological processes and pathways. Biological processes are integrated, complex, and involve substantial "cross-talk ${ }^{\mathrm{n}}$ amongst various systems. Proteomics - the study of what proteins are actually present in a cell at a given point in time-can be studied alone in a narrow kinetics sense; however, if a greater understanding must be obtained of what controls the proteome (e.g., the dynamics), then other cellular systems must also be studied and integrated. As a step in this direction, the High-Performance Mass Spectrometry Facility will expand into the area of metabalomics and thus increase its protein analysis capabilities for complexes to include protocols and searches for the RNA members of these systems that are so important in establishing their correct activation. The facility will also expand and improve its data analysis capabilities to be "intelligently"directed. Currently, the largest failing in the ability to perform database searches for peptides is where the peptide target is not present in the database due to poor database annotation, database errors, or possible strain variations on the system being studied. Better "mining" of the information present in the spectra has great potential to improve analytical capabilities. Finally, every effort will be made to keep facility equipment at the state of the art, equally emphasizing separation technology — one of the signature features of this facility.

\section{Facility Staff}

\section{Harold R. Udseth}

Technical Lead (509) 376-3698

harold.udseth@pnl.gov

Kimberly S. Korenkiewicz

Administrative Secretary (509) 373-0765

kimberly.korenkiewicz@pnl.gov

Dave J. Anderson

Scientist

(509) 376-7898

david.anderson@pnl.gov
Heather M. Mottaz

Scientist

(509) 376-4869

heather.mottaz@pnl.gov

Ronald J. Moore

Technologist

(509) 376-2721

ronald.moore@pnl.gov

Rui Zhang

Scientist

(509) 376-3200

rui.zhang@pnl.gov 


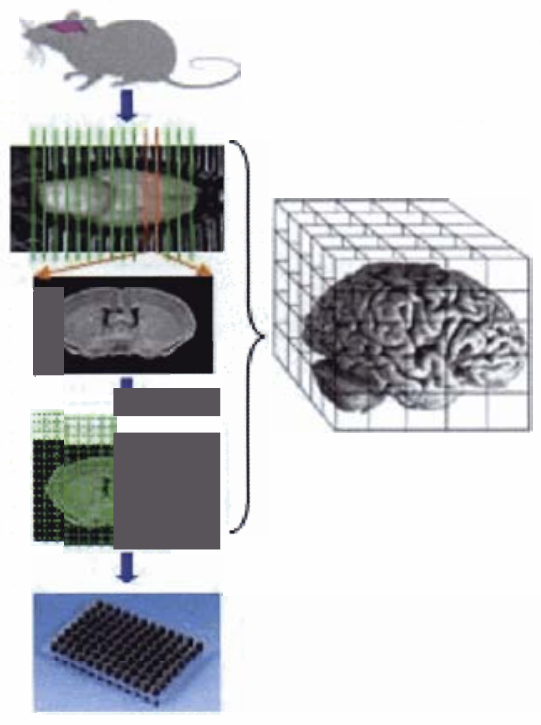

Kim K. Hixson

Scientist

(509) 373-6170

kim.hixson@pnl.gov
Rui Zhao

Scientist

(509) $376-7332$

rui.zhao@pnl.gov

\section{Key Staff}

Key staff, besides those listed above, include David G. Camp, Joshua N. Adkins, Carrie D. Goddard, Marina Gritshenko, Mary S. Lipton, Christophe D. Masselon, and Ljiljana Pasa-Tolic.

\section{Three-Dimensional Proteomic Mapping of the Mouse Brain}

W Qian, ${ }^{(a)} \mathrm{H}$ Wang, ${ }^{(a)}$ VA Petyuk, ${ }^{(a)}$ M H Chin, ${ }^{(b)}$ DJ Smith, ${ }^{(b)}$ DG Camp, ${ }^{(a)}$ and RD Smith ${ }^{(a)}$

(a) Pacific Northwest National Laboratory, Richland, Washington

(b) University of California at Los Angeles David Geffin School of Medicine, Los Angeles, California

Figure 4-71. Schematic of the voxelation process. The mouse is sacrificed and the brain extracted. The brain is sectioned into layers and each layer is then cubed by a rectangular cutting grid.

Understanding how the genome gives rise to the staggering three-dimensional complexity of the mammalian brain is one of the major goals for post-genomic biology. To address this problem, it will be necessary to spatially map transcripts, proteins, and the networks they form in the brain at a genome-wide level. In this study, we investigated the potential for using high-throughput, high-sensitivity proteomics and the voxelation technology for three-dimensional proteome mapping of the mouse brain. Added value for the use of the mouse model system for three-dimensional mapping arises from the existence of mouse models for a number of neurodegenerative diseases. Figure 4-71 shows the overall voxelation process. Mouse brain tissue is directly sampled as cubes using a three-dimensional coordinate system. 'The voxelation was performed by first cutting the brain into 10 coronal sections and then each section was cut into -60 cubes with each cube of $-0.75 \mu \mathrm{L}$ (Singh et al. 2003). "The voxels were directly put into 96 well plates for automated sample processing and high-throughput proteome analyses.

'The final phase of peptide/protein identification will use high-throughput liquid chromatography-FTICR analyses based on the accurate mass and time (AMT) tag approach. 'This approach requires the generation of an A M T tag database as a first step and as validation of the approach. We have completed the first phase validating the approach and have generated an extensive A M T tag database for the mouse brain. This required the development of novel and highly efficient enrichment techniques for cysteine-containing peptides. 'The samples were then fractionated and analyzed using multidimensional liquid chromatography followed by online tandem mass spectrometry (MS/MS) detection using ion trap mass spectrometers. The database currently contains more than 35,000 
different A M T tags, covering-6900 different mouse brain proteins and is the most extensive protein database ever generated for the mouse brain. "The proteins detected were sorted according to the subcellular component in which they would be found according to annotations in the genome. Figure 4-72 shows the coverage of identified brain proteins based on subcellular components. 'The complete coverage of mouse brain subcellular locations in the AMT tag database provides a solid basis for future quantitative analyses of brain voxels.

Success of the next phase of the project requires the analyses of single voxel samples using an automated microscale sample process and extremely sensitive liquid chromatography-mass spectrometry detection using EMSL's FTICR mass spectrometers. 'These requirements have presented procedural challenges that have been overcome. We have developed a simple protocol for tissue lysis and protein digestion, and initial automated sample processing was successful. With this protocol, the typical amount of peptides recovered per voxel was -20 _g. With our in-house-developedcapillary liquid chromatography separations, this is enough for both liquid chromatography-MS/MS and liquid chromatographymass spectrometry analyses. Figure 4-73 shows the successful analyses of two adjacent single voxels using liquid chromatography-FTICR. For each voxel, approximately 3000 peptides and 1000 proteins were identified using AMT tags from our database. "The automated sample processing is still being optimized and improvements in the number of proteins detected per voxel are expected. The next step will be quantitative analyses of large number of voxels for threedimensional mapping. The normalization and quantitation approaches are still being refined to optimize the confidence of the identification and minimize the error bars on the protein quantities. We anticipate that the initial generation of three-dimensional protein abundance patterns (e.g., for normal and Parkinson disease mouse models) will provide novel insights.

\section{Citation}

Singh RP, VM Brown, A Chaudhari, AH Khan, A Ossadtchi, DM Sforza, AK Meadors, SR Cherry, RM Leahy, and DJ Smith. 2003. "High-Resolution Voxelation Mapping of Human and Rodent Brain Gene Expression." Journal of Neuroscience Methods 125(1-2):93-101.

\title{
Probing the Function of Proteins by Multidimensional Mass Spectrometric Analysis of Breast Cancer Membrane Proteomes
}

\author{
AJ Patwardhan, (a) EF Strittmatter, ${ }^{(b)}$ DG Camp, ${ }^{(b)}$ RD Smith, ${ }^{(b)}$ and \\ MG Pallavicin ${ }^{(\mathrm{a})}$ \\ (a) University of California San Francisco, San Francisco, California \\ (b) Pacific Northwest National Laboratory, Richland, Washington
}

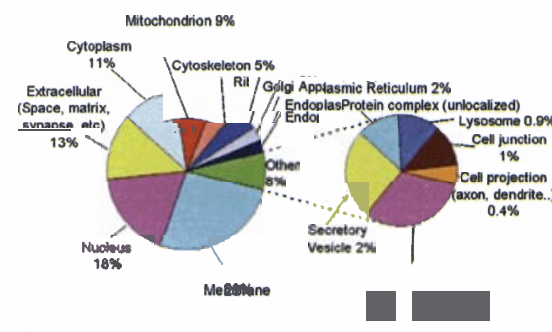

Figure 4-72. Distribution of proteins in our database amongst the subcellular components according to annotations of the mouse genome.

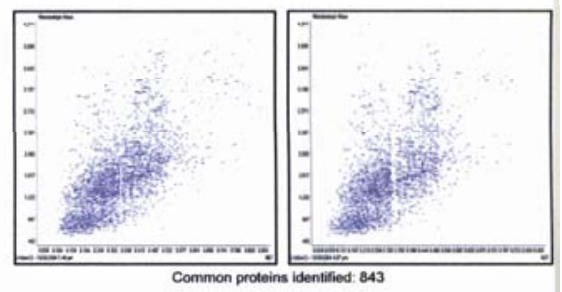

Figure 4-73. Two-dimensionalplots for the liquid chromatography-FTICRanalysts from two neighboring single voxels 


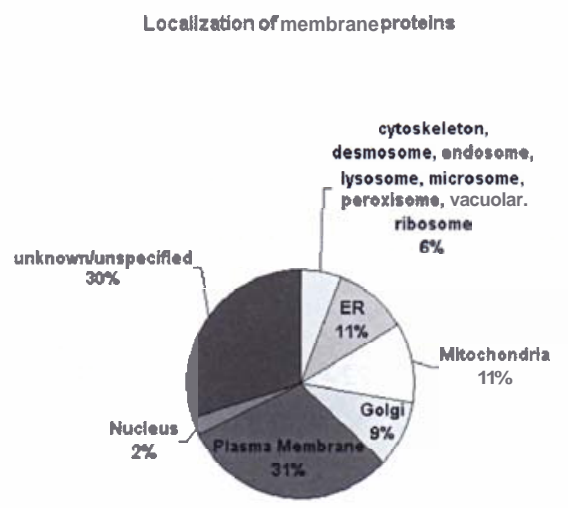

Figure 4-74. Summary of localization information for 965 membrane proteins identified from MS/MS
Proteins associated with plasma cell membranes participate in multiple cellenvironment interactions including cell-cell communications, cell-extracellular interactions, locomotion and migration, and cell signaling. Membraneassociated proteins account for 70 percent of all known pharmaceutical drug targets and are targets for a number of new drug and antibody-based therapeutics in cancer. We undertook a study to define the membrane proteome of breast cancers. To capture the maximum number of breast cancer-associated proteins, we analyzed the membrane proteome of a panel of breast cancer cell lines using a combination of subcellular fractionation, separation by strong cation exchange chromatography, and reversed-phase capillary liquid chromatography followed by MS/MS. The proteins identified were investigated further in an effort to identify plasma membrane-associated proteins (PMAPs) and groups of proteins overrepresented in the breast cell-line panel. Additional information derived from gene expression data sets were used to identify proteins that may have a potential relevance to breast cancer.

Four breast cancer cell lines [SKBR-3, MDA-MB-231, BT-474, and an MCF7-c18 transfectant cell line over-expressing the ERBB2 receptor tyrosine kinase (RTK)] were cultured and used to make up the breast cancer panel. Membrane-enriched fractions were prepared separately for all four cell lines anu then pooled into a single mixture. All sample aliquots combined to produce 202 fractions, which produced 724,566 MS/MS spectra. 'This corresponded to a total of 42,250 high-scoring peptides matching 2344 proteins listed in the September 2003 freeze of the human International Protein Index database (http://www.ebi.ac.uk/IPI/IPIhelp.html). Among all proteins identified, 28 percent had less than 10 percent sequence coverage, 65 percent had 20 to 50 percent sequence coverage, and 6 percent had sequence coverages greater than 60 percent. Membrane-bound proteins were further subcategorized into cellular organelles based on the gene ontology, UniProt, and InterPro databases (Figure 4-74) and ambiguities were resolved using PSORTII.

'The transmembrane-spanning region of proteins is frequently lost in membraneenriched preparations due to the inability to keep hydrophobic regions of the protein solubilized. A decrease in the number of transmembrane domains (TMDs) may suggest that portions of the membrane protein embedded within the lipid bilayer are not captured efficiently with our isolation procedure or analytical strategies. "The number of TMDs was predicted using the software package TM HMM 2.0 prediction server and was used to extrapolate the number of TMDs expected for 2198 (94 percent) of the identified proteins. Percent protein coverage varied greatly among both membrane-containing and membrane-free proteins and no significant relationship between the number of TMDs and percent protein coverage was observed. However, sequence coverage above 40 percent was generally not seen in proteins with multiple TMDs.

These data suggest that there may be some bias introduced against hydrophobic portions of the membrane that may have been lost during sample preparation or analysis. 'The majority of proteins (66 percent, 1455) had 0 TMDs, while 34 percent (743) had between 1 and 23 TMDs as predicted by the TM H MM 2.0 server. Given that global genomic analysis predicts that 20 to 30 percent of all open reading frames encode integral membrane proteins (including both 
plasma and intracellular membranes), our results indicate that any bias against membrane proteins is relatively small.

Querying of the UniGene database revealed more than 1905 unique genes represented by this set of proteins. 1525 ( 80 percent) of these unique genes (identified from all three aliquots) were mapped to the gene ontology database and classified according to molecular function and cellular component categories. A combination of both molecular function and cellular component categories yielded 312 proteins that were associated with the plasma membrane, as evidenced by both functional and localization information. Because gene ontology categories are often incomplete and ambiguous, several proteins were manually inspected for association with the plasma membrane. Manual inspection revealed an additional 40 PMAPs. Most proteins in the subset of 352 PMAPs were associated with signal transducer activity, binding, and transporter activities. "The subcategorization of our data set according to the gene ontology database resulted in many proteins putatively involved in immune response, apoptosis, catalytic activity, and several other cellular processes. According to the PFAM database, 20 protein sequence domain/families were well represented (more than five member proteins) in our collection of PMAPs. Many of these domains include well-conserved transmembrane-bound domains and included several groups of proteins known to be membrane-associated. Among those identified was the immunoglobulin domain (-100 residues); the fibronectin type III domain (-100 residues); the epidermal growth factor-like domain (30-40 residues); the SH3 domains (-50 residues); the WD domain G-beta repeat ( -40 residues); the FERM ( -150 residues) domain; the PDZ domains (80-90 residues); the tubulin domains; the sterile alpha motif (more than 70 residues); the cadherin domain; and the low-density lipoprotein receptor domain. Well-represented protein families included members of the ADP-ribosylation factor family, the ABC transporters, the Ras family of green fluorescence protein (GTP)ases, the guanine nucleotide binding G-protein family, and the tetraspanin family.

Using the procedure described by Hosack et al. (2003), protein groupings based on functional domains in several protein signature databases were used to determine whether any populations of proteins were abundant in our sample. Two protein categories (as defined in the PFAM and SMART) contained a significantly $(p<0.01)$ higher number of proteins in our sample than would be expected by random chance. Four proteins belonging to the SNF-7 homologues (PFAM, PF03357) domain were identified in our sample. This family of proteins is involved in formation of multivesicular bodies, which play a critical role in the recycling and degradation of membrane proteins (i.e., activated cell-surface receptors). 'Thirteen proteins belonging to the PDZ/DHR/GLGF (SMART, SM00228) domain were also identified. Members of this group of proteins are thought to direct the targeting of signaling molecules to submembranous sites. 'The PDZ/DHR/GLGF domain group includes proteins frequently associated with the plasma membrane, a compartment where high concentrations of phosphatidylinositol 4,5-bisphosphate (PIP2) are found. They interact directly with several ephrin-related RTKs, the ERBB2 RTK, and with C-terminal sequences derived from other transmembrane receptors. 
SAGE tag numbers directly reflect the abundance of mRNAs in vivo and can therefore be used as an accurate and quantitative measure of global gene expression profiles in breast tissue. Using archived, publicly available SAGE data sets, we analyzed differences in gene expression between pools of normal and cancerous breast tissue. Differential gene expression is inferred from the relative frequency of tags occurring in pools of cancerous SAGE libraries vs. pools of normal SAGE libraries. The expression profiles for 34 PMAPs showed at least a threefold difference in expression between pools of normal and cancerous breast tissue. Several of the proteins over-expressed in cancerous breast tissue have previously been investigated with regard to breast cancer. The RTK protein ERBB2 has been well documented as a prognostic marker in breast cancer and was over-expressed in all the cancerous tissues surveyed compared to normal tissue. Notably, the myristoylated alanine-rich C-kinase substrate protein, putatively involved in the activation of the ERBB2 signaling pathway, also was over-expressed in tumor tissue. Other over-expressed proteins in tumor tissue included the peripheral benzodiazepine receptor, the ephrin receptor EPHBB3, the melanoma-associated antigen D2, beta platelet-derived growth factor receptor, the semaphorin $3 \mathrm{C}$ receptor precursor, dystroglycan and Claudin-3. Several of the PMAPs over-expressed in breast cancer tissue had little or no previous work done in relation to breast cancer. 'These proteins include the tyrosine-protein kinase-like 7 precursor, the EFG receptor kinase substrate, the FK506-binding protein 3 , the calcium-binding protein $\mathrm{p} 22$, the serine/threonine-protein kinase 25, the Erythrocyte band 7 integral membrane protein, the Cadherin EGF receptor, the retinoic acid-induced 3 protein, the DKFZp761D0211 hypothetical protein, and the GDNF family receptor alpha 1 precursor.

Among the proteins under-expressedin cancerous compared to normal breast tissue are several that show a surprisingly large disparity between both pools. The Annexin A1 protein is under-expressed in all cancerous tissues compared to normal. This protein belongs to a family of calcium-dependent, phospholipidsbinding proteins that are preferentially located on the cytosolic face of the plasma membrane and is thought to have potential anti-inflammatory activity and act as endothelial adhesion molecules. Other proteins under-expressed in cancer tissue include the CD44 and CD63 antigens, the GTP-binding protein, the jagged-1 protein, and the ephrin receptor EPHA2.

In summary, our study identified a large collection of PMAPs from a breast cancer cell-line panel using high-throughput $\mathrm{ms} / \mathrm{ms}$ techniques. Several tools allowed us to create a membrane proteome profile of this set of cell lines. In addition, we were able to combine gene expression data with proteomic data to identify several PMAPs that may play important roles in the initiation and progression of breast carcinomas. Because we analyzed a limited number of specimens, additional experiments using high-throughput techniques, such as mRNA in situ hybridization or immunohistochemical analysis on tissue-microarrays, are required to determine how commonly these genes are differentially expressed. Additional analysis of these genes and the biochemical pathways in which they are involved will not only further our understanding of breast oncogenesis, but will also provide new and valuable targets for translational research. 
Hosack DA, G Dennis, BT Sherman, HC Lane, and RA Lempicki. 2003.

"Identifying Biological Themes Within Lists of Genes with EASE." Genome Biology 4(10):R70.

\section{Comparative Proteomics and Cytochrome c Location Determination in Geobacter sulfurreducens}

\author{
KK Hixson, ${ }^{(a)}$ YR Ding, ${ }^{(b)}$ MS Lipton, (a) and DR Lovley(b) \\ (a) Pacific Northwest National Laboratory Richland, Washington \\ (b) University of Massachusetts, Amherst, Massachusetts
}

Geobacter sulfurreducens is an anaerobic metal-reducing soil bacteria that possesses beneficial capabilities ranging from bioremediation through the reduction of heavy metals and radionuclides to electricity production through electron transport to electrodes on its outer surface (Bond and Lovley 2003; Lovley 1995). 'The genome sequence of G. sulfurreducensreveals a number of novel capacities that have not been previously characterized (Methe et al. 2003). To understand the mechanisms involved in bioremediation and electricity production, it is first necessary to determine the proteins that are expressed under different physiological conditions in different locations within the cell. To this end, the global proteome of G. sulfurreducens was characterized by analysis of proteins from cells grown under eight different conditions with ultrahigh-pressureliquid chromatography, and mass spectrometry using the AMT tag methodology. Cluster analysis using OmniViz ${ }^{\mathrm{TM}}$ revealed the reproducibility of triplicate analyses and relevant protein abundance changes among cells grown with different electron acceptors and environmental stress. 2968 gene products (approximately 86 percent of total putative open reading frames in the genome) were detected including 91 putative c-type cytochrome gene products. Comparative cluster analysis using normalized abundance values showed relevant proteins expressed under eight different environment culture conditions, including perturbations of heat shock and oxygen exposure (Figure 4-75). Highly abundant proteins expressed under iron (III) reduction included cytochromes c (OmcB, PpcE, OmcS and MacA), a glycosyltransferase, lipoproteins, a metal efflux protein, an ABC transporter, an extracellular solutebinding protein, signal transduction and response regulators, and conserved hypothetical proteins. Proteins specifically related to oxygen reduction for energy conservation and heat-shock response were additionallyidentified.

Fundamental to this organism's metabolism and metal-reducing capabilities are the transport of electrons from the inner cytosol to the external bacterial surface. At the heart of electron transport is a chain of proteins with heme moieties, which allow for the movement of electrons from one protein to another. "These

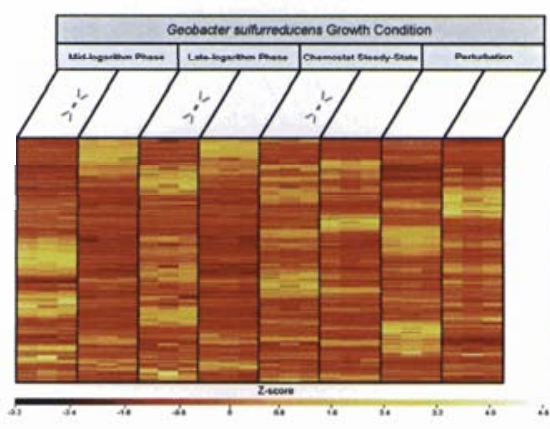

Figure 4-75. Cluster analysis of 2968 proteins expressedin eight different culture conditions. Lighter-colored areas represent proteins expressed in a higherthan-average abundance because of that culture condition. 


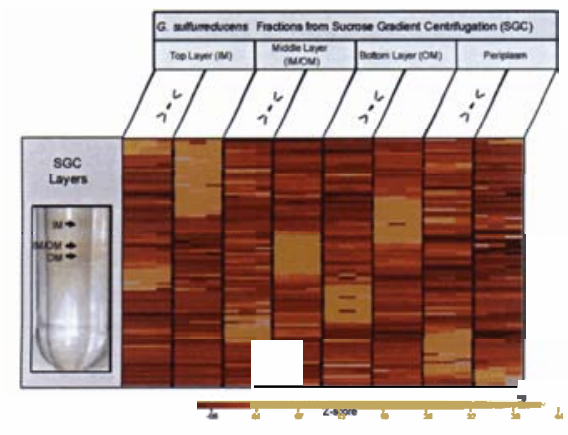

Figure 4-76. Cluster analysis of 91 c-type cytochromes revealing the predominate location of these proteins from proteins isolated into inner, outer, and periplasmic locations within the bacteria G sulfurreducens. Lighter-colored regions revealed proteins that show a greater-thanaverage normalized abundance level for that culture condition. proteins are classified as cytochromes. Unique in this organism is the genetic coding of more than 110 putative c-type cytochrome genes (Methe et al. 2003). The identity and location of cytochrome $\mathrm{c}$ gene products were determined by first separating the inner and outer membranes by sucrose gradient centrifugation and subsequently identifying the proteins and performing a cluster analysis, which revealed the predominate location of $91 \mathrm{c}$-type cytochromes (Figure 476). 'Ihis information may reveal the paths of fundamental electron transport between proteins in G. sulfurreducens, thus revealing the mechanisms involved in bioremediation and electricity production.

\section{Citations}

Bond DR, and DR Lovley. 2003. "Electricity Production by Geobacter Sulfurreducens Attached to Electrodes." Applied andEnvironmentalMicrobiology 69(3):1548-1555.

Lovley DR. 1995. "Bioremediation of Organic and Metal Contaminants with Dissimilatory Metal Reduction."Journal of Industrial Microbiology 14(2):85-93.

Methe BA, KE Nelson, JA Eisen, IT Paulsen, W Nelson, JF Heidelberg, D Wu, M Wu, N Ward, MJ Beanan, RJ Dodson, R Madupu, LM Brinkac, SC Daugherty, RT DeBoy, AS Durkin, M Gwinn, JF Kolonay, SA Sullivan, DH Haft, J Selengut, T M Davidsen, N Zafar, O White, B Tran, C Romero, HA Forberger, J Weidman, H Khouri, TV Feldblyum, TR Utterback, SE Van Aken, DR Lovley, and CM Fraser. 2003. "Genome of Geobacter Sulfurreducens: Metal Reduction in Subsurface Environments." Science 302(5554):1967-1969.

\section{Global Quantitative Proteomics Without Isotope Labeling}

R Fang, (a) D A Elias, (a) M E Monroe, (a) Y Shen, (a) CD Goddard, (a) SJ Callister, (a) RJ Moore, ${ }^{(b)}$ YA Gorby, ${ }^{(a)}$ JK Fredrickson, ${ }^{(a)}$ MS Lipton, ${ }^{(a)}$ and RD Smith ${ }^{(a)}$

(a) Pacific Northwest National Laboratory, Richland, Washington

(b) W.R. Wiley Environmental Molecular Sciences Laboratory, Richland, Washington

We are investigating an alternative approach for the relative quantification of protein abundances using liquid chromatography-mass spectrometry technologies. Current approaches based on stable isotopic labeling of proteins, or components of proteins known as peptides, either chemically or metabolically can be cost prohibitive and often result in a fewer number of proteins being detected by liquid chromatography-mass spectrometry. "This alternative approach does not require isotopic labeling, but requires a linear correlation between the amount of the analytes and their peak areas measured by mass spectrometry. 'Ihis has been demonstrated with simple mixtures of a few analytes (Tang et al. 2004; Cech and Enke 2001; Voyksner and Lee 1999), and it has been suggested that this linear correlation can be obtained for more complex mixtures of analytes using a sufficiently low flow rate and 
a small amount of sample. With such conditions, the ionization of analytes using electrospray ionization approaches an optimum (-100 percent) ionization efficiency (Tang et al. 2004; Cech and Enke 2001).

This alternative approach, abbreviated as AMT-time tag non-isotopic labeling quantification, uses the previously developed A M T tag procedure for highthroughput confident identification of peptides (Smith et al. 2002; Lipton et al. 2002). We applied the AMT-time tag non-isotopic labeling quantification approach to the metal-reducing bacteria Shewanella oneidensis strain MR-1 cultured in bioreactors under both aerobic ( 20 percent dissolved $\mathrm{O}_{2}$ tension) and suboxic $(<0.1$ percent 02$)$ conditions. Arbitrary abundances of peptides from proteolytic digests of proteins extracted from collected samples were measured using liquid chromatography-FTICR spectroscopy operated at a conventional flow rate. Normalization of the peptide abundance across all liquid chromatography-FTICR analyses to correct for possible systematic bias was performed.

Experimental and instrument reproducibility of the AMT-time tag nonisotopic labeling quantification approach was demonstrated using scatter plots. An example displayed in Figure 4-77 (top) plots an instrument replicate of abundances of peptides from one collected sample versus an instrument replicate of abundances of peptides from a duplicate sample. These samples were collected simultaneously while the bioreactor operated under an aerobic condition. We observed good reproducibility among all instrument replicates from these duplicate samples as indicated by root sum square (R2) values ranging from 0.95 to 0.96 . Figure 4-77 (bottom) plots an instrument replicate from one bioreactor operating under an aerobic condition against an instrument replicate from a different bioreactor operating under a suboxic condition. Here, $\mathrm{R} 2$ values among instrument replicates were significantly lower, ranging from 0.65 to 0.67 , indicating that the abundance of certain peptides - and therefore the proteins they represent-were different between culture conditions.

\section{Citations}

Cech NB, and CG Enke. 2001. "Practical Implications of Some Recent Studies in Electrospray Ionization Fundamentals." Mass Spectrometry Reviews 20(6):362-387.

Lipton MS, L Pasa-Tolic, GA Anderson, DJ Anderson, DL Auberry, JR Battista, MJ Daly, J Fredrickson, KK Hixson, H Kostandarithes, C Masselon, LM Markillie, RJ Moore, MF Romine, Y Shen, E Stritmatter, N Tolic, HR Udseth, A Venkateswaran, KK Wong, R Zhao, and RD Smith. 2002. "Global Analysis of the Deinococcus Radiodurans Proteome by Using Accurate Mass Tags." Proceedings of the National Academy of Sciences of the United States of America 99(17):11049-11054.

Smith RD, GA Anderson, MA Lipton, L Pasa-Tolic, Y Shen, TP Conrads, TD Veenstra, and HR Udseth. 2002. "An Accurate Mass Tag Strategy for Quantitative and High-Throughput Proteome Measurements." Proteomics 2(5):513-523.

Tang K, JS Page, and RD Smith. 2004. "Charge Competition and the Linear Dynamic Range of Detection in Electrospray Ionization Mass Spectrometry." Journal of the Society for Mass Spectrometry 15(10):1416-1423.

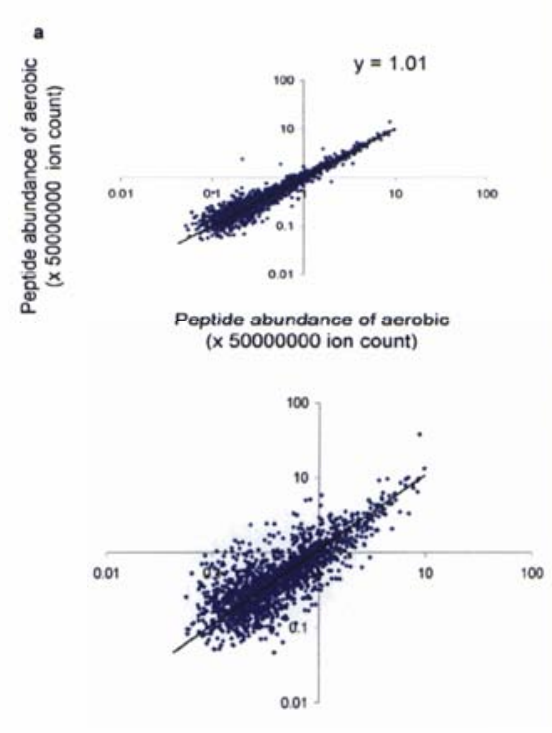

Figure 4-77. (top) Scatter plot of abundances of peptides from an instrument replicate from one collected sample versus abundances of peptides from an instrument replicate taken from a duplicate sample. Each sample was collected from the same bioreactor operating under an aerobic environment. (bottom) Scatter plot of abundances of peptides from an instrument replicate from one bioreactor operating under an aerobic environment versus abundances of peptides from an instrument replicate from a different bioreactor operating under a suboxic environment. 


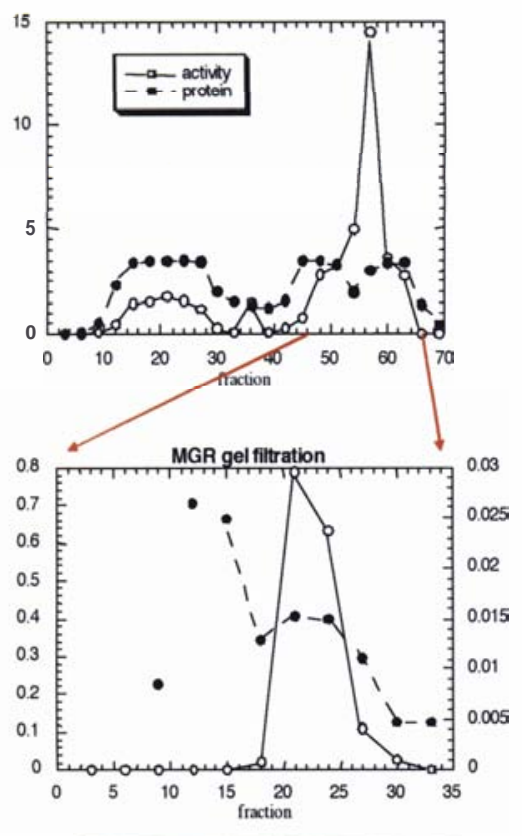

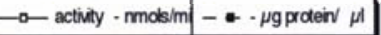

Figure 4-78. Isolation of methyglyoxyl reductase activity using anion exchange and sizeexclusion chromatography.
Voyksner RD, and H Lee. 1999. "Investigating the Use of an Octupole Ion Guide for Ion Storage and High-Pass Mass Filtering to Improve the Quantitative Performance of Electrospray Ion Trap Mass Spectrometry." Rapid Communications in Mass Spectrometry 13(14):1427-1437.

\section{Illumination of Previously Unidentified Proteins with Specific Enzyme Activity Using Mass Spectrometry}

\author{
RC Barry, (a) DH Kohl, ${ }^{(b)}$ D G Camp, (a) and RD Smith ${ }^{(a)}$ \\ (a) Pacific Northwest National Laboratory, Richland Washington \\ (b) Washington University, St. Louis, Missouri
}

Researchers are collaborating to establish a mass spectrometry-based approach for identifying those proteins responsible for specific enzymatic activity, but whose identity was previously unknown. While the availability of complete genome sequences opens the door to important biological advances, much of the actual understanding of cellular systems and the roles of its constituents will be based on techniques in proteomics (which we define here as the study of the functions of proteins). Current gene annotation techniques fall short of accurately identifying the functions of proteins within an organism. Many proteins, as identified by open reading frames, have unknown function and are listed as 'hypothetical' proteins. Further, there are many biological processes that are carried out by organisms whose protein players are completely unknown. Often, many of the enzymes that participate in biochemical pathways are completely unknown, and in some cases have been identified from previously classified hypothetical proteins. Developing a rapid and sensitive method for associating enzymatic activities with proteins from a specific gene would greatly accelerate our ability to gain an understanding of the structure and function relationships of enzymes and the biological pathways used by organisms to promote survival.

Present methods typically use a series of purification steps to isolate fractions containing specific enzymatic activity. The proteins from these fractions are then separated with Coomassie Blue-stained bands using sodium dodecyl sulfate polyacrylamide gel electrophoresis. Identification of the enzymes responsible for the activity is inferred from the intensity of the stained bands. "Theoretically, the more purification steps one uses, the greater the purity of the compound that is producing the activity and the higher the specific activity detected. Whole-cell lysates, for example, may be fractionated using anion exchange chromatography. Those fractions that contain the activity can be isolated and further resolved using size-exclusion chromatography, where again those fractions containing specific activity can be isolated for further analysis. We are studying the reduction of methylglyoxal in yeast cells. Extensive work at Washington 
University using the conventional approach described above identified the open reading frame that expresses the nicotinamide adenine dinucleotide phosphate hydrogen-dependentenzyme responsible for this activity.

'These results are shown in Figure 4-78, where the open circles show the specific activity for methylglyoxal reductase (nicotinamide adenine dinucleotide phosphate hydrogen-dependent). 'This was increased 122-fold going from anion exchange chromatography (upper figure) to size-exclusion chromatography (lower figure). In this preliminary work, the proteins are extracted from each of the fractions that demonstrate increased enzymatic activity, digested with trypsin, and then analyzed using ultra-sensitiveliquid chromatography-FTICR mass spectrometry. "The concentration-dependence of the resulting peptides, ionized by the electrosprayionization process, is used to determine the identity of the protein(s), which similarly demonstrate an increase in their concentration in solution. Figure 4-78 shows the results for the enzyme methylglyoxal reductase as the solid circles. They show general agreement with the enzymatic activity and point out more general issues associated with linking protein concentrations with enzymatic activity. Often co-factors are required to activate an enzyme and only a complete inventory of all biologically important molecules present in a fraction can lead to a complete understanding.

With the liquid chromatography-FTICR technology, all other proteins present can also be detected as possible candidates. Once a candidate list is produced, further experiments can be performed to validate protein function, such as using glutahione tranferase-fusion tags to over express the protein of interest in yeast to provide protein for biochemical assays. Additional experiments are underway to minimize the number of different purification steps required by collecting and analyzing the protein content of the individual fractions from a single purification run to identify the protein(s), which dramatically increase in concentration as well as specific activity.

\title{
Comparative Proteomics of Yersinia pestis
}

\author{
KK Hixson, (a) JN Adkins, ${ }^{(a)}$ M E Monroe, ${ }^{(a)}$ SL McCutchen-Maloney, ${ }^{(b)}$ and \\ MS Lipton ${ }^{(a)}$
}

(a) Pacific Northwest National Laboratory, Richland, Washington

(b) Lawrence Livermore National Laboratory, Livermore, California

'The ability to respond adequately to a biological threat requires detailed understanding of the threat agent to determine the appropriate response. Proteins serve as the basis of all mechanisms in biological systems, and understanding the changes in protein expression pattern is crucial to understanding their physiological mechanisms. Yersinia pestis, the causative agent of plague, is a known bioterrorist and biowarfare threat agent (Perry and Fetherston 1997). Virulence-associated proteins of Y.pestis can be induced or repressed in culture by adjusting conditions that mimic the environments of the flea vector $\left(26^{\circ} \mathrm{C}\right)$ and the human host $\left(37^{\circ} \mathrm{C}\right.$ with and without calcium). 


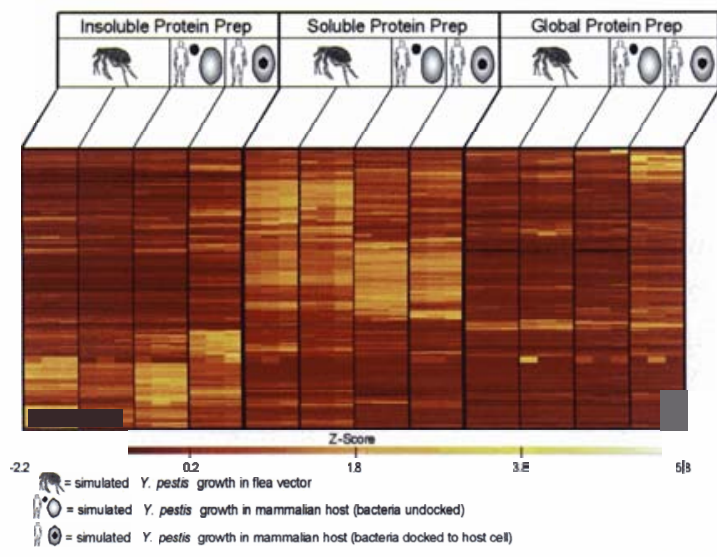

Insight into the virulence mechanisms of $Y$. pestis can therefore be furthered by the characterization of protein expressed under contrasting temperatures and calcium concentration.

Our approach to global proteomics is based on protein digestion followed by peptide identification by mass spectrometry using the AMT tag methodology (Smith et al. 2002). 'This approach provided comparative normalized abundance values for 962 proteins identified with two or more unique peptides (Figure 4-79).

Furthermore, cluster analysis was performed on 146 proteins identified from similarity clusters of Figure 4-79 that displayed similar abundance patterns as nine known virulent proteins
Figure 4-79. Comparative cluster analysis of expressed proteins (open reading frames, or ORFs) detected using abundance measurements from liquid chromotography-mass spectrometry-FTICR analysis. Lighter colors highlight proteins that are higher in abundance than the average for that culture condition. Darker colors highlight proteins that display a lower-than-average abundance for that culture condition.
(Figure 4-80). 'These results have revealed reproducible measurements between triplicate analyses with known and novel protein abundance changes by conditions that mimic virulent and non-virulent bacterial states. Virulent protein expression is clearly observed between bacterial growth from $26^{\circ} \mathrm{C}$ to $37^{\circ} \mathrm{C}$ with calcium (bacteria's initial entry into mammalian host) and at $37^{\circ} \mathrm{C}$ without calcium (bacterial docking with host cell).

Many of the proteins that were found to cluster in a similar pattern with the nine known virulent proteins in Figure 4-80 were novel hypothetical proteins from both the chromosome and plasmids. It is these novel proteins that most likely also play a role in virulence. 'These newly identified proteins thus make perfect candidates as biomarkers for protein-based detection technologies and better post-infection treatment and vaccine development.

Figure 4-80.

Cluster analysis of 146 proteins that show similar abundance changes to nine known virulent proteins.

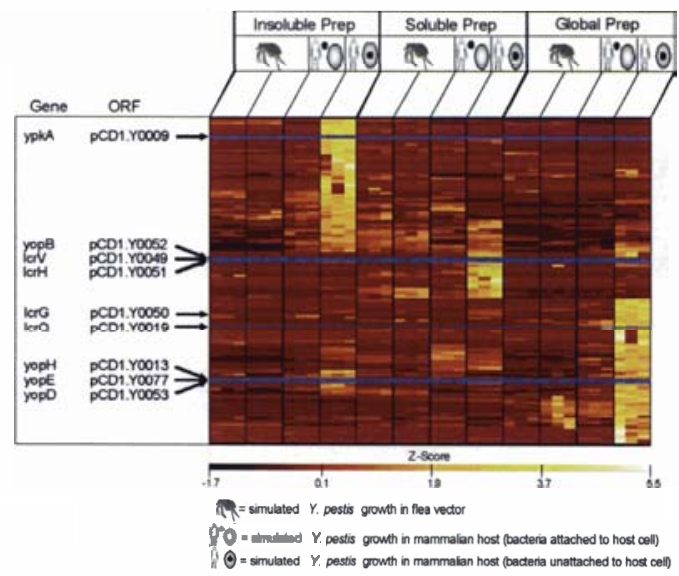

\section{Citations}

Perry RD, and JD Fetherston. 1997. "Yersinia pestis—Etiologic Agent of Plague." ClinicalMicrobiologyReviews 10(1):35-66.

Smith RD, GA Anderson, MS Lipton, L Pasa-Tolic, Y Shen, T P Conrads, TD Veenstra, and HR Udseth. 2002. "An Accurate Mass Tag Strategy for Quantitative and HighThroughput Proteome Measurements." Proteomics 2(5):513-523. 


\title{
Secretory and Membrane Proteins of Pseudomonas aeruginosa
}

\author{
W Xiao, ${ }^{(a)}$ RD Davis, ${ }^{(a)}$ and M Mindrinos ${ }^{(a)}$ \\ (a) Stanford University, Stanford, California
}

Pseudomonas aeruginosa is a gram-negative bacterium of increasing importance because it is one of the top three opportunistic pathogens in humans, causing premature death in patients with cystic fibrosis, HIV infection, organ transplants, or cancer (Quinn 1998). It is the most common cause of various nosocomial infections, including sepsis in burned or extensively injured patients, and is resistant to antibiotics. Consequently, there is significant interest in the proteins responsible for this virulence, particularly those of the cellular membrane. The phenotypic resistance of gram-negative bacteria is a direct consequence of the complex structure of their cell envelope, which acts as a barrier and prevents drug molecules from reaching their target sites or increases their active efflux, primarily mediated by various classes of integral membrane proteins (Nikaido 1998). It has been indicated that antibiotic resistance is due to integral outer membrane protein channels (porins) and membrane protein complexes known as multidrug efflux pumps that transport antibiotic and biocide molecules out of the cell (Nikaido 1998).

Large-scale analysis of hydrophobic integral membrane proteins from complex protein mixtures is an important and challenging aspect of mass spectrometrybased membrane proteomics. Although, large-scale proteomic studies based on in-gel digestion of proteins separated using two-dimensional polyacrylamide gel electrophoresis (2-D PAGE) have recently shown significant improvements in protein coverage, very hydrophobic integral membrane proteins are generally not amenable using this approach primarily due to the issues related to the insolubility of these proteins and precipitation occurring at their isoelectric points (Nouwens et al. 2000: Santoni et al. 2000).

\section{Results and Discussion Membrane Protein Identification}

The membrane subproteome of P. aeruginosa was probed using two techniques we developed specifically for mass spectrometry identification of highly hydrophobic integral membrane proteins (Blonder et al. 2002; Goshe et al. 2003) and is outlined in Figure 4-81. Both techniques were used concomitantly in the current investigation in order to achieve a more comprehensive analysis of membrane proteins than could be obtained by using only one approach. When analyzed by SEQUEST, all the collected MSIMS spectra of both experiments produced a total of 9951 fully tryptic peptides, of which 2727 peptides were unique. From these data, a total of 786 protein identifications were observed: 623 proteins from the unlabeled sample and 163 proteins from the labeled sample. The set of 786 identified proteins contains 544 identified from the unlabeled sample and 84 from labeled sample, corresponding to 707 unique proteins. Using results presented in a previous report describing a global analysis 
of yeast proteins using multidimensional chromatography and database searching of MS/MS spectra using SEQUEST (Peng et al. 2003), the percentage of false positives associated with our study was determined to be no greater than 10 percent. To increase the confidence of positive identification of cysteinyl-labeled peptides to greater than 90 percent, we added the additional requirement of the presence of at least two label-specificions in the MS/MS spectrum (Borisovet al. 2002). On the basis of these numbers, the overall enrichment of proteins containing at least one T MD relative to all those identified was 42 percent and 44 percent for the labeled and unlabeled sample, respectively.

Importantly, all four subunits of succinate dehydrogenase $s d h A$ (PA1583), $s d b B$ (PA1584), $s d h C$ (PA1581), and $s d h D$ (PA1582) predicted by the genome sequence were confidentlyidentified in the unlabeled sample out of which $s d h C$ and $s d h D$ are very hydrophobic. Subunits $s d h A, s d b B$, and $s d h C$ were also identified in the labeled aliquot. Together, these results reflect the efficiency of the techniques used in extracting, solubilizing, and labeling highly hydrophobic transmembrane peptides, which lead to unambiguous identification of highly hydrophobic integral membrane proteins from complex mixtures using conventional one-dimensional (liquid chromatography-MS/MS analysis.

\section{Transferases and Cytochromes}

Prolipoprotein diacylglyceryl transferase (PA0341) is a hydrophobicintegral membrane protein with four mapped TMDs. This protein, not previously identified in $\mathrm{P}$. aeruginosa, was characterized by identifying four peptides. The first peptide (SFFQLMDFIAPLVPIGLGAGR) is very hydrophobic and completely covers the second mapped TMD. PA0341 is an essential factor for the growth, division, and viability of bacterial cells at nonpermissive temperatures. Homologues were identified in Escherichia coli, Salmonella typhymurium, and Haemophilus influenzae (Gupta et al. 1993). PA0341 is encoded by the lgt gene and is involved in fatty acid and phospholipid metabolism, translation, and post-translational modifications (Qi et. al. 1995). As a cytoplasmic membrane enzyme, it is a part of the sequential catalysis that involves prolipoprotein signal peptidase and the $\ln t$ gene which encodes for apolipoprotein N-acyl transferase (PA3984) (Gupta et al. 1993). PA3984 has six mapped TMDs and is involved in the formation of lipid-modified proteins. The protein modification is achieved by transferring the diacylglycerol group from phosphatidyl-glycerol to the sulfhydryl group of a cysteinyl residue present at the C-terminal portion of the signal sequence of the protein (Gupta et al. 1993).

MdoH and $m d o G$ genes are necessary for the synthesis of membrane-derived oligosaccharides that occupy the periplasmic space of gram-negative bacteria and for glucosyl transferase activity. MdoHcodes for an integral membrane protein expressing 76 percent homology with the $m d o H$ gene of E. coli whose protein spans the cytoplasmic membrane and is required for the expression of disease symptoms and development of hypersensitive reaction on nonhost plants (Loubens et al. 1993). The $m d o H$ protein of P. aeruginosa (PA5077) was detected by four peptides from both the unlabeled and labeled samples. Three proteins (PA1554, PA1557, and PA4430) from the cytochrome super family were also 
identified by hydrophobic peptides. These proteins were not previously identified in $\mathrm{P}$. aeruginosa or other prokaryotes according to the current $\mathrm{P}$. aeruginosa Community Annotation Project Database. In addition, 14 other proteins from this super family were identified. Additional proteins, not previously identified in P. aeruginosa until applying our mass spectrometry-based approach, include NADH dehydrogenase I chain H (PA2643) and heat shock protein PA2643, whose homologues were characterized previously in E. coli exhibiting 84 percent and 63 percent sequence similarity, respectively (Weidner et al. 1993; Kornitzer et al. 1991).

\section{Antibiotic Resistance, Adaptation, and Protection Proteins}

Since the most important targets of antibacterial agents are located within the cytoplasmic membrane or cytoplasm, the outer membrane presents a natural barrier to antibiotics and biocides, preventing their access to target sites (Denyer et al. 2002). Porins or channel-type proteins are important factors of antibiotic resistance of $\mathbf{P}$. aeruginosa because they prevent the passage of hydrophilic antibiotics via a size exclusion mechanism, whereas the dense lipopolysaccharride bilayer of the outer membrane slows down the penetration of hydrophobic macromolecules inside the cell (Poole 2002). However, the lower permeability of the outer membrane alone cannot completely protect the microbe; therefore, additional cellular mechanisms are necessary to create the high level of antibiotic resistance exhibited by P. aeruginosa (Poole 2002). Hydrolytic enzymes possessing lactamase activity are omnipresent within the periplasmic space. We confidently identified a probable penicillin amidase (PA1032) by detecting multiple peptides. Notably, this enzyme was not previously characterized in P. aeruginosa and could serve as a viable pharmacological target.

\section{Conclusion}

'This work presents a global qualitative analysis of the membrane subproteome of $\mathrm{P}$. aeruginosa strain PAO1 in which hydrophobic membrane proteins are confidently identified on a comprehensive, large-scale basis using $\mu$ liquid chromatography-MS/MS. Several biologically and medically significant classes of integral membrane proteins involving antibiotic resistance, adaptation, and susceptibility were identified in this investigation regardless of their hydrophobicity and membrane location.

\section{Citations}

Blonder J, MB Goshe, RJ Moore, L Pasa-Tolic, CD Masselon, MS Lipton, and RD Smith. 2002. "Enrichment of Integral Membrane Proteins for Proteomic Analysis Using Liquid Chromatography-Tandem Mass Spectrometry." Journalof Proteome Research 1(4):351- 360.

Borisov OV, MB Goshe, TP Conrads, VS Rakov, TD Veenstra, and RD Smith. 2002. "Low-Energy Collision-InducedDissociation Fragmentation Analysis of CysteinylModified Peptides." Analytical Chemistry 74(10):2284-2292.

Denyer SP, and JY Maillard. 2002. "Cellular Impermeability and Uptake of Biocides and Antibiotics in Gram-Negative Bacteria." Journal of Applied Microbiology 92:35S-45S. 
Goshe MB, J Blonder, and R D Smith. 2003. “Affinity Labeling of Highly Hydrophobic Integral Membrane Proteins for Proteome-Wide Analysis." Journal of Proteome Research 2(2):153-161.

Gupta SD, K Gan, MB Schmid, and HC Wu. 1993. "Characterization of a Temperature-Sensitive Mutant of Salmonella Typhimurium Defective in Apolipoprotein N-Acyltransferase." The Journal of Biological Chemistry 268(22):16551-16556.

Kornitzer D, D Teff, S Altuvia, and AB Oppenheim. 1991. "Isolation, Characterization, and Sequence of an Escherichia coli Heat Shock Gene, htpX." Journal of Bacteriology 173(9):2944-2953.

Loubens IL, A Debarbieux, J M Bohin, JP Lacroix, and JP Bohin. 1993. "Homology Between A Genetic Locus (Mdoa) Involved in the Osmoregulated Biosynthesis of Periplasmic Glucans in Escherichia coli and a Genetic Locus (Hrpm) Controlling Pathogenicity of Pseudomonas Syringae." Molecular Microbiology 10:329-340.

Nikaido H. 1998. "Multiple Antibiotic Resistance and Efflux." Current Opinion in Microbiology 1(5):516-523.

Nouwens AS, SJ Cordwell, M R Larsen, M P Molloy, M Gillings, M D P Willcox, and BJ Walsh. 2000. "Complementing Genomics with Proteomics: The Membrane Subproteome of Pseudomonas aeruginosa Pao1." Electrophoresis 21(17):3797-3809.

Peng J, JE Elias, CC Thoreen, LJ Licklider, and SP Gygi. 2003. "Evaluation of Multidimensional Chromatography Coupled with Tandem Mass Spectrometry (LC/LC-MS/MS) for Large-Scale Protein Analysis: The Yeast Proteome."Journal of Proteome Research 2(1):43-50.

Poole K. 2002. "Mechanisms of Bacterial Biocide and Antibiotic Resistance." Journal oJ Applied Microbiology 92:55S-64S.

Qi HY, K Sankaran, K Gan, and HC Wu. 1995. "Structure-Function Relationship of Bacterial Prolipoprotein Diacylglyceryl Transferase: Functionally Significant Conserved Regions." Journal of Bacteriology, 177(23):6820-6824.

Quinn JP. 1998. "Clinical Problems Posed by Multiresistant Nonfermenting GramNegative Pathogens." Clinical Infectious Diseases 27:S117-S124.

Santoni V, M Molloy, and T Rabilloud. 2000. "Membrane Proteins and Proteomics: Un Amour Impossible?" Electrophoresis 21(6):1054-1070.

Weidner U, S Geier, A Ptock, T Friedrich, H Leif, and H Weiss. 1993. "The Gene Locus of the Proton-Translocating NADH: Ubiquinone Oxidoreductase in Escherichia coli: Organization of the 14 Genes and Relationship Between the Derived Proteins and Subunits of Mitochondria1 Complex I." Journal of Molecular Biology 233(1):109-122. 


\title{
Proteomic analysis of Wild Type and Soil-Colonization Deficient Strains of Pseudomonas fluorescens
}

\author{
SB Levy, ${ }^{(a)}$ H M Mottaz, ${ }^{(b)}$ MS Lipton, ${ }^{(c)}$ I Lopez-Hernadez, ${ }^{(a)}$ and EA \\ Robleto ${ }^{(a)}$ \\ (a) Tufts University School of Medicine, Boston, Massachusetts \\ (b) W.R. Wiley Environmental Molecular Sciences Laboratory, Richland, \\ Washington \\ (c) Pacific Northwest National Laboratory, Richland, Washington
}

\begin{abstract}
A significant step towards developing effective genetically modified microbes for bioremediation purposes is understanding the ecology of release, growth, and establishment of populations in soil. In Pseudomonas fuorescens, a microbe widely used for enhancing soil characteristics, our laboratory identified a locus (AdnA) affecting several cell processes important for its behavior in soil. Mutants deficient in AdnA are defective in biofilm formation, motility, and attachment to sand and seeds. Of more importance, mutants affected in AdnA did not colonize and persist in soil as well as the wild type (Marshall et al. 2001). AdnA shows homology to FleQ from Pseudomonas aeruginosa, a transcription factor of the NtrC/NifA family that is involved in flagella synthesis (Casaz et al. 2001). 'This type of regulator is Sigma 54-dependent, known to activate transcription upon changing environmental conditions. Therefore, by identifying traits affected by $\mathrm{Adn} A$, we shall gain insights into how to improve soil colonization, persistence, and genetic determinants affecting soil colonization by comparing proteomes from the wild type and an AdnA-deficientmutant. Recently, we began to characterize the regulon controlled by AdnA using a genetic approach (Robleto et al. 2003).
\end{abstract}

In order to develop a more complete picture of the genes controlled by AdnA, we aim to compare the genes expressed in wildtype $P$. fluorescens (Pf0-1) with those expressed in an AdnA deletion mutant (PfO-2x). Genetic evidence shows that AdnA-dependent genes are expressed at significantly lower levels in Pf0$2 \mathrm{x}$ than Pf0-1 (Robleto et al. 2003). 'Thus, protein extracts from Pf0-2x are likely to contain no peptides activated and/or peptides that are repressed by the AdnA transcription factor. Proteornic technologies offer promise in this kind of approach due to the broad coverage now being obtained, and the fact that translated products are compared, rather than just transcribed products revealed using microarray technology. In combination, microarray and proteomic approaches will allow a full description of genes under control of AdnA.

Wildtype (Pf0-1) and mutant (Pf0-2x) P. fluorescens were grown under conditions known to favor expression of AdnA-dependant genes in the wildtype strain. Proteins have been extracted from cytoplasmic extracts, membrane fractions, and whole cells, and were subjected to trypsin digestion. Using 
FTICR mass spectrometry, the peptides are being individually identified, and by computational means have been compared to the draft genome sequence of $P$. fuorescens. This approach has enabled us to identify 763 expressed genes in Pf0-1, and 1271 in Pf0-2x, using peptides found in cytoplasmic extracts. Peptides found in membrane fractions revealed 437 expressed genes in Pf0-1 and 290 in Pf0-2x. Comparison of the data sets shows that, of the cytoplasmic proteins, 451 present in the Pf0-1 extract are absent from Pf0-2x. Among membrane proteins, $318 \mathrm{Pf0}-1$ proteins are absent from Pf0-2x. 'These data are highly likely to overestimate the number of proteins whose expression is governed by AdnA, probably as a result of incomplete coverage of the proteome at this time. However, examination of the proteins absent from $\mathrm{PfO}-2 \mathrm{x}$ will allow some candidate AdnA-regulated genes to be identified. It is known that AdnA regulates motility, and the proteomic data point to a number of flagella and chemotaxis proteins not previously known to be part of the AdnA regulon. For example, seven methyl-accepting chemotaxis proteins are unique to the membrane fraction of Pf0-1. Our previous genetic study only identified two (Robleto et al. 2003).

While the AdnA regulon remains to be completely defined, an additional benefit from the proteomic data is the confirmation that a number of previously annotated "hypothetical ${ }^{\mathrm{n}}$ genes are indeed expressed (e.g., 55 "hypotheticals" from the membrane of PfO-1). Moreover, proteins with no matches in databases have also been shown to be expressed (e.g., 84 from the Pf0-1 membrane fraction). These data will be used to aid in the thorough annotation of the genome sequence of PfO-1 when it is completed.

\section{Citations}

Casaz P, A Happel, J Keithan, DL Read, SR Strain, and SB Levy. 2001. “The Pseudomonas Fluorescens Transcription Activator Adna is Required for Adhesion and Motility." Microbiology 147(2):355-361.

Marshall B, EA Robleto, R Wetzler, P Kulle, P Casaz, and SB Levy. 2001. “The Adna TranscriptionalFactor Affects Persistence and Spread of Pseudomonas Fluorescens under Natural Field Conditions." Applied Environmental Microbiology 67(2):852-857.

Robleto EA, I Lopez-Hernandez,MW Silby, and SB Levy. 2003. "Genetic Analysis of the Adna Regulon in Pseudomonas Fluorescens: Nonessential Role of Flagella in Adhesion to Sand snd Biofilm Formation."Journal of Bacteriology 185(2):453-460. 


\title{
Identification of the Labeled Residue(s) of SecB Protein
}

\author{
LL Randall, (a) J Crane, ${ }^{(a)}$ and S Martinovic ${ }^{(b)}$ \\ (a) University of Missouri, Columbia, Missouri \\ (b) W.R. Wiley Environmental Molecular Sciences Laboratory, Richland, \\ Washington
}

SecB, a small tetrameric cytosolic chaperone in Escherichia coli, facilitates the export of precursor polypeptides by maintaining them in a nonnative conformation and passing them to SecA, which is a peripheral member of the membrane-bound translocation apparatus. To elucidate the interaction between $\mathrm{SecB}$ and the unfolded precursor, we are mapping the molecular path that the precursor takes when it binds SecB. 'The structure of protein SecB is shown in Figure 4-82. 'The use of site-directed mutagenesis to systematically replace single residues with cysteine coupled with EPR allows scanning through SecB, identifying sites of interaction. EPR technology requires an unpaired electron, which we provide by labeling the introduced cysteinyl residue in SecB with methanethiosulfonate, a stable nitroxide spin label. Ideally, in our experimental approach, one would remove all native cysteines and introduce a single cysteine at the site of interest. However, we have been unable to replace two of the four native cysteines and maintain the protein in an active form. 'Thus, each of our constructs has three cysteines. It is imperative that we know confidently that we quantitatively label the introduced cysteine and not the native cysteines.

Mass spectrometry is the technique of choice to identify and quantify amino acid modifications in proteins. 'These are challenging experiments, but they are the only means of unambiguouslydetermining if the targeted cysteine residue has been labeled correctly. In order to confidentlyidentify the labeled residue position, a series of experiments are underway in EMSL's High-Performance Mass Spectrometry Facility. In the first experiment, the molecular mass of intact proteins was measured on a 7-tesla FTICR mass spectrometer in order to confirm the number of labels per protein. This is a quick means of determining that only one residue has been labeled and that the molecular weight of the label is correct. An example of this process is represented by the spectra in Figure 4-83. 'The mass spectrometer detects a series of multiply protonated versions of the protein as shown in the inset in the figure. To obtain a quantitative representation, each one of these charge-state spectra must be deconvoluted to the molecular weight of the uncharged protein (zero charge state). 'The resulting zero charge spectra in the figure show the methanethiosulfonate-labeledand non-methanethiosulfonate versions of the SecB protein and easily allow the number of labels per protein to be determined.

In subsequent experiments, proteins were digested by trypsin to the peptide level. Liquid chromatography-massspectrometry and liquid chromatographyMS/MS experiments on using LCQ ion trap and 9.4-tesla FTICR mass spectrometers enabled identification of peptides and the labeled residue. Results cov-
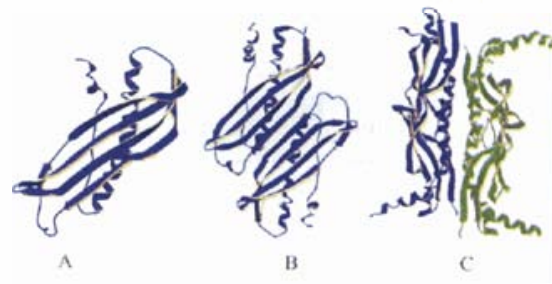

Figure 4-82. Structure of the Hemophilus influenzae SecB protein. Ribbon representation of the monomer $(\mathrm{A})$; dimer $(\mathrm{B})$; and tetramer $(\mathrm{C})$ where the view is orthogonal to the view of dimer.

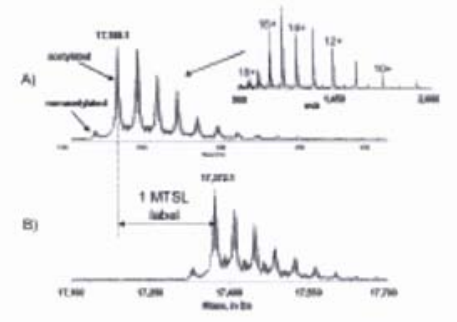

Figure 4-83. Zero charge ESI FTICR mass spectra of $\mathrm{Sec} B$ protein: wild type $(A)$ and methanethiosulfonate labeled $\operatorname{SecB}(B)$. The inset shows chargestate distribution in the mass spectrum that was deconvoluted to $\mathrm{A}$. 
ered 100 percent of protein sequences in all analyzed proteins (that have replaced single residue) and showed which cysteine residue(s) were modified.

\section{Proteomic Profiling of Deinococcus Radiodurans}

MS Lipton ${ }^{(a)}$

(a) Pacific Northwest National Laboratory, Richland, Washington

\begin{tabular}{|c|c|c|c|c|}
\hline Category & Size & $\begin{array}{c}\text { Predicted } \\
\text { ORFs }^{1}\end{array}$ & $\begin{array}{c}\text { Observed } \\
\text { ORFs }\end{array}$ & $\begin{array}{c}\text { Percent } \\
\text { Coverage }\end{array}$ \\
\hline Total & $3.29 \mathrm{Mbp}$ & 3116 & 2585 & 83 \\
\hline Chromosome & $12.65 \mathrm{Mbp}$ & 2633 & 2154 & 83 \\
\hline Chromosome & $2412 \mathrm{kbp}$ & 369 & 304 & 82 \\
\hline Mega Plasmid & $177 \mathrm{kbp}$ & 145 & 100 & 68 \\
\hline Small Plasmid & $46 \mathrm{kbp}$ & 40 & 27 & 67 \\
\hline 10pen Reading Frames
\end{tabular}

'The $\mathrm{D}$. radiodurans strain $\mathrm{R} 1$ is a gram-positive, nonpathogenic bacterium whose 3.1 Mbase genome consists of two chromosomes, one megaplasmid and one plasmid [3960]. D. radiodurans has an extraordinary ability to tolerate both acute and chronic exposures to high levels of ionizing radiation-it can survive 5000 to 15,000

Table 4-1.

$D$. radiodurans Proteome Coverage
Gy of acute ionizing radiation (depending on culture conditions) and grow continuously under $60 \mathrm{~Gy} / \mathrm{hr}$. Our analysis used the 3116 protein-encoding open reading frames predicted by the TIGR annotation (ftp://ftp.tigr.org/pub/data/ dradiodurans/GDR.pep) (we exclude from this analysis 71 open reading frames predicted to contain frame shifts). However, the annotation consists of the best prediction of open reading frames encoded by an organism based on comparison to open reading frames identified in other organisms and on the codon usage. 'The proteomic measurements provide a physical validation that the open reading frames actually encode a protein.

More than 400 liquid chromatography-MS/MS analyses of peptides from collective culture conditions yielded tentative identifications for more than 70,000 peptides having a SEQUEST score above 2. Our FTICR measurements verified with high confidence 26326 of these (AMT). In order to confirm the presence of an open reading frame in our analysis, we required that at least two mass tags were identified for an open reading frame and that those mass tags were seen in at least three spectra in the FTICR mass spectrometer. Using these criteria, we identified 2585 open reading frames from D. radiodurans, representing 83 percent of the predicted open reading frames (Table 4-1) for Deinococcus radiodurans corresponding to the broadest proteome coverage for an organism achieved to date. More than 90 percent of the open reading frames are identified if only one mass tag is required for open reading frame identification. In a single FTICR analysis, the masses for $-1,500$ AMTs are typically detected, corresponding to -700 open reading frames (depending on the culture condition), and 15 to 20 percent of the $\mathrm{D}$. radiodurans proteome.

The proteomic profile of D. radiodurans contained open reading frames assigned to every functional category. Figure 4-84 is a representation of the distribution 
of proteins into these functional categories. "The size of the pie slice indicates the number of proteins from the annotated genome that fall into the category indicated. Each pie piece has been shaded to indicate the percentage of proteins from that category that has been identified by the proteomic profiling. Not surprising, the categories associated with housekeeping functions, protein synthesis, amino acid synthesis, and cell envelope had representation for the most part of over 90 percent. Hypothetical and conserved hypothetical proteins are proteins whose expression has not been previously confirmed by physical methods. The proteomic profiling of D. radiodurans confirmed the expression of 88 percent and 80 percent of the conserved hypothetical and hypothetical proteins, respectively. In confirming the expression of these proteins, these open reading frames represent possible targets for further study.

Coverage of functional category members can be used to predict the expression of specific metabolic pathways. Predicted proteins involved in various D. radiodurans metabolic pathways were commonly identified with multiple different AMTs, suggesting their high abundance. We verified the expression of all the predicted proteins corresponding to the vacuolar type (V-type) proton ATP synthase, as well as the predicted components of the organism's

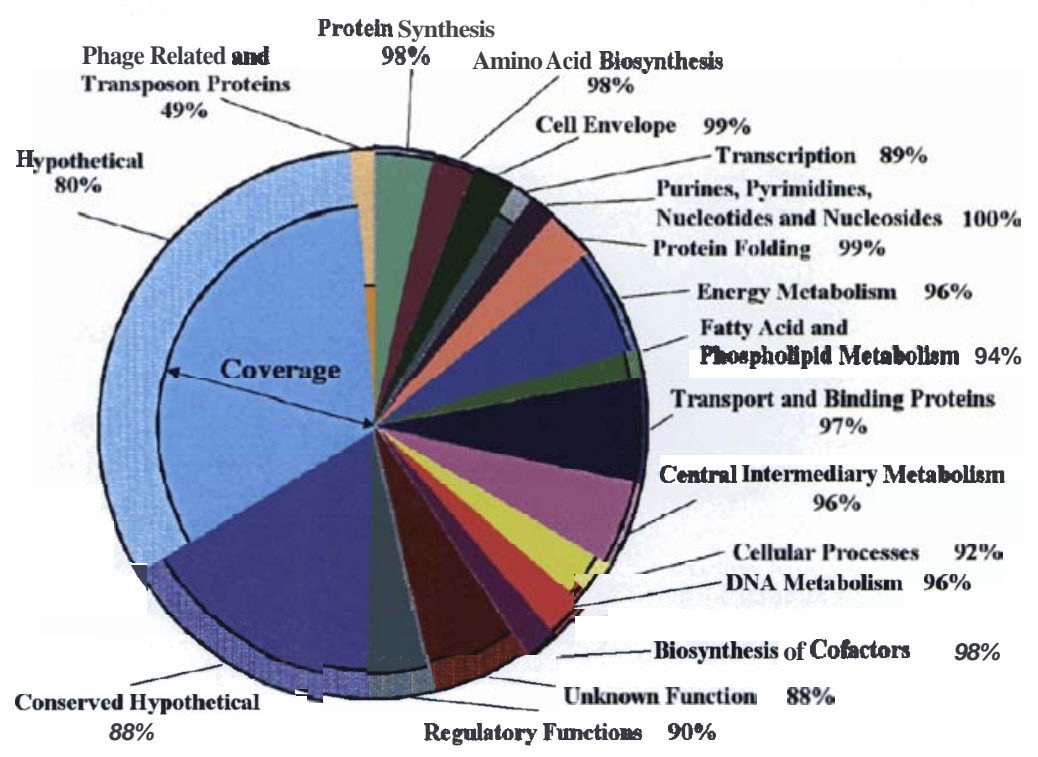
TC A cycle enzymes. In addition, 80 percent of the predicted proteins involved in glycolysis, and the pentose phosphate shunt were detected. A slightly smaller fraction of electron transport proteins were identified (several are integral membrane proteins). The patterns of expression for all the open reading frames (data not shown) illustrates that many predicted proteins associated with "housekeeping" functions are expressed under all conditions evaluated. Approximately 32 percent and 16 percent of the open reading frames from the $\mathrm{D}$. radiodurans database are predicted to be hypothetical and conserved hypothetical, respectively. We identified 80 percent of these hypothetical proteins and 88 percent of the conserved hypothetical proteins. 


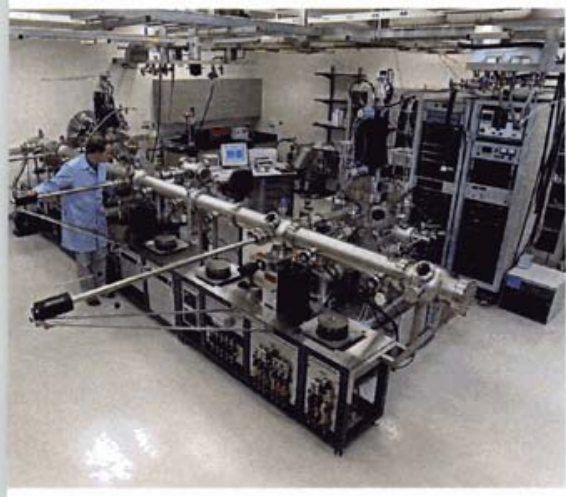

Molecular beam epitaxy, as performed at EMSL's Interfacial and Nanoscale Science Facility. This system is used to grow and characterize thin crystalline films of oxides and ceramics to obtain a detailed understanding of surface reactions.

\subsection{5}

\section{Interfacial and Nanoscale Science Facility}

'The Interfacial and Nanoscale Science Facility is a world-class resource for scientific expertise and instrumentation focused on the study of interfacial phenomena and nanoscience and technology. Activities in the facility address national needs in environmental cleanup and restoration, waste management, pollution prevention, clean energy production, and national security through research that specializes in preparation and characterization of suitable materials with various sizes, developing fundamental understanding of their chemical and physical properties along with chemical reaction mechanisms, especially at the surfaces and interfaces. 'The range of scientific expertise and instrumentation within the facility provides a unique environment for research in areas such as surface and interfacial science; nanomaterials synthesis and characterization for various applications; complex interfacial catalysis; environmental interfaces, including aerosols and minerals; material properties and interfaces including radiation effects, defect properties and interactions, ionic and atomic transport, and electronic and magnetic properties; and areas within microanalytical science, such as chemical and biosensing and nanobiotechnology.

'Ihe Interfacial and Nanoscale Science Facility and its scientific staff provide a broad range of instrumentation, laboratory capabilities, and expertise. Instrumentation is available for material synthesis using thin film deposition and chemical synthesis, ion beam processing and analysis, various aspects of surface and bulk characterization, analytical chemistry, separations, advanced electrochemical synthesis, ultrahigh vacuum and real world catalysis and surface chemistry, and microfabrication. Capabilities include an accelerator facility for material modification and analysis using ion beams along with interface characterization; ambient and ultrahigh vaccum scanning probe microscopes; electron microscopy and x-ray analysis; spectroelectrochemistry; high-spatial/ energy resolution surface analysis; catalyst preparation, characterization, and reaction engineering; a fully equipped clean room for microfabrication, microanalytical systems development and testing laboratories; inorganic, organic, polymer, and biochemical synthesis and characterization facilities; a full complement of thin-film deposition and characterization facilities; and fully equipped analytical support laboratories. "The combination of surface and interface characterization techniques that provide high spatial, depth, and energy resolution for a broad array of methods is unmatched anywhere in the world. Many systems are coupled directly to film growth chambers, and samples can be moved among many ultrahigh vacuum systems under controlled environment without exposure to air using portable transfer systems.

\section{Representative Research Activities}

Researchers assigned to the Interfacial and Nanoscale Science Facility perform innovative research in the areas of surface and interfacial chemistry, advanced materials synthesis and characterization, and microanalytical science. Activities emphasize research relevant to the four DOE mission areas-science, 
energy resources, environmental quality, and national security-and to operation of a world-class user facility for scientific problem-solving that supports the science mission. Facility staff also play a major role in the continued success of EMSL by providing support, training, and collaboration to onsite users. During the past seven years, research activities at the facility have focused on four major thrust areas: 1) films and interphases; 2) surface chemistry and catalysis; 3) material interfaces; and 4) microsensors, microfluidics, and new biotechnologies. Facility researchers and users focus their efforts in these four areas, with research on the following topics:

- Structural and chemical properties of model single-crystaloxide and complex mineral surfaces and interfaces

- High-dielectric materials, oxide dilute magnetic semiconductors, and oxide catalysts

- Nanoscale materials, such as oxide quantum dots, nanofilms of magnetic and oxygen ion-conducting oxides, and buried nanoclusters in oxides

- Fundamental understanding of reaction mechanisms at oxide and mineral surfaces and interfaces

- Development of new microanalytical separations and sensing principles, tools, and testing

- Single-enzyme nanoparticles, enzymes in nanostructured matrices, understanding the dynamics of these materials

- Environmental studies involving waste separations, structural and chemical stability of waste forms under different radiation and chemical environment, and atmospheric aerosols

- Analysis and characterization using fully equipped analytical laboratories and characterization facilities.

\section{Research Capabilities}

- 'Thin-film deposition capabilities, including oxygen plasma-assisted molecular beam epitaxy, metal organic chemical vapor deposition, sputter deposition and sol-gel deposition

- Surface analysis suite that consists high-spatial resolution XPS, Auger electron spectroscopy, and TOF ion mass spectrometry

- Electron microscopy suite that consists of high-resolution transmission electron microscopes and scanning electron microscopes

- Scanning probe microscopy capabilities, including various ambient and ultrahigh vacuum scanning tunneling microscopes and atomic force microscopes and scanning Auger microscope

- Ion-beam processing and analysis capabilities that include various ion implantation and ion beam analysis methods

- Nanobiotechnology capabilities

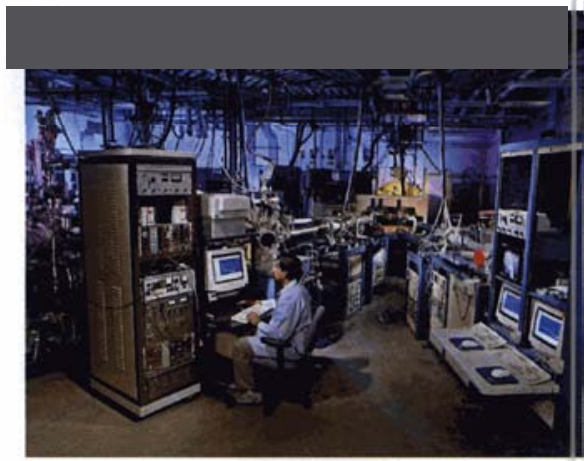

The Ion Beam Materials Analysis Laboratory of the Interfacial and Nanoscale Science Facility consists of a 3-MV tandem ion accelerator, two ion sources, three beam lines, and four end stations. All of the ion beam processing and analysis capabilities area available in this accelerator facility. 
- Surface science and catalysis laboratory that consists of ultrahigh vacuum systems with several surface science techniques

- Catalytic reactors, including an RXM 100 catalyst test stand

-XRD laboratory with special applications, thin film characterization, and powder diffraction instrumentation

- Microfabrication capabilities

- Chemical and biological sensing capabilities

- Other analytical and characterization laboratories.

\section{Recent Upgrades}

\section{Scanning Probe Microscopy Capability}

A great need exists to establish experimental tools that can acquire single-site chemical information which will lead to important new knowledge in several areas. In particular, heterogeneous catalysis is strongly site specific, and for important catalytic materials such as metal oxides, reactions involve various regular and defect sites. Ideally, one would like to obtain chemically specific information on a sub-nanometer scale. 'Thus, the variable ultrahigh vacuum noncontact atomic force microscope/scanning tunneling microscope purchased by the Interfacial and Nanoscale Science Facility in 2004 is ideal for these experiments. "The facility has added many surface science capabilities to this instrumentation, including XPS; Auger electron spectroscopy, and plasma, sputter cleaning, and deposition capabilities.

\section{Liquid Delivery System for Metalorganic Oxide Chemical Vapor Deposition (MOCVD)}

The MOCVD capability of the Interfacial and Nanoscale Science Facility is oversubscribed because of demands for instrument time from external users, PNNL programs, and industrial clients. Users of this capability have produced extensive scientific output, including many publications in internationally peerreviewed journals. Because the MOCVD capability at EMSL has tremendous potential to provide high-quality scientific output, the capability is being equipped with a new liquid delivery system to repair a failing liquid vaporizer system. 'The new liquid delivery system has been installed and tested. The MOCVD instrumentation was made available to users in mid-March 2005.

\section{Heavy Ion Elastic Recoil Detection Analysis}

The scientific objective is to develop a high-resolution T OF elastic recoil detection analysis capability in the ion beam material analysis laboratory (acceleratorfacility) at EMSL. 'This technique will provide researchers with simultaneous detection and absolute quantification of hydrogen, carbon, nitrogen, oxygen, and other light elements as a function of depth in complex matrices containing heavy elements. Because it is a powerful method for investigating elemental concentrations in surface regions, this capability will be effectively applied in many different areas, including characterization of oxide 
thin films for optical, magnetic, and catalysis applications, and characterization of environmental and biological samples. Development of this capability will be complete during Fiscal Year 2005.

\title{
Enhanced Deposition Capability in the Molecular Beam Epitaxy Laboratory
}

To meet researcher demands, the state-of-the-art oxygen plasma-assisted molecular beam epitaxy system located in the Interfacial and Nanoscale Science Facility's Molecular Beam Epitaxy Laboratory was upgraded with additional effusion cells, bringing the total number of individually controlled molecular beam epitaxy sources to eight. In addition, the plasma source is being upgraded with differential pumping capabilities.

\section{Transmission Electron Microscope with Cryo-Stage}

To meet the demands of biological and biogeochemical research, including activities associated with the Membrane Biology and Biogeochemistry Scientific Grand Challenges, a dedicated transmission electron microscope with cryo-stage is being procured.

\section{Future Direction}

\author{
Researchers of the Interfacial and Nanoscale Science Facility will continue \\ to focus on innovative research in the areas of surface and interface \\ chemistry, advanced material synthesis and characterization, nanoscience and \\ nanobiotechnology, and catalysis.
}

In the near future, Interfacial and Nanoscale Science staff will participate and support the research activities associated with EMSL's Membrane Biology and Biogeochemistry Scientific Grand Challeriges. To support these activities, the facility will complete development of TEM capabilities with cryo-stage and actively incorporate other capabilities to address the scientific issues associated with these challenges. In addition, facility staff will participate and support activities associated with the Catalysis CAT. This will include developing new capabilities, including 1) a high-spatilil and energy resolution, aberrationcorrected TIM with dual focus ion beam; 2) capabilities for sample preparation and SEM, and 3) an environmental cell to develop a fundamental understanding of reaction mechanisms while the reactions occur. In addition, a next-generation high-pressure catalysis system with ultrahigh vacuum surface science capabilities and an ultrahigh vacuum scanning tunneling microscopy/atomic force microscope for site-specific chemistry/catalysis research will be developed.

At present, nanomaterial characterization is a challenge, even with use of most of the well-developed surface and bulk characterization capabilities. The Interfacial and Nanoscale Science Facility will continue to pursue development of new nanomaterial characterization capabilities and innovative research in synthesis and characterization of nanoscale materials for solid oxide fuel cell and thermoelectric power applications. 
applications and oxide quantum dots for photocatalysis applications and will pursue new capabilities to advance research in these areas. In addition, facility staff will continue to develop ion beam capabilities for trace element detection in atmospheric aerosols and biological samples and for characterization of radiation detection materials. These capabilities will help to address some of the scientific issues associated with homeland security.

\section{Facility Staff}

S. Thevuthasan

Staff Scientist

Technical Lead

(509) 376-1375

theva@pnl.gov

Barbara L. Diehl

Administrative Secretary

(509) 376-1518

barb@pnl.gov

\author{
A. Scott Lea \\ Senior Research Scientist \\ (509) 376-9145 \\ scott.lea@pnl.gov \\ Igor V. Lyubinetsky \\ Senior Research Scientist \\ (509) 376-5220 \\ igor.lyubinetsky@pnl.gov \\ David E. McCready \\ Research Scientist \\ (509) 376-9648 \\ david.mccready@pnl.gov \\ Yanwen Zhang \\ Senior Research Scientist \\ (509) 376-3429 \\ yanwen.zhang@pnl.gov
}

Mark H. Engelhard

Research Scientist

(509) 376-1664

mark.engelhard@pnl.gov

Daniel J. Gaspar

Senior Research Scientist

(509) 376-2413

daniel.gaspar@pnl.gov

Laxmikant V. Saraf

Senior Research Scientist

(509) 376-2006

laxmikant.saraf@pnl.gov

V. Shutthanandan

Senior Research Scientist

(509) 376-2708

shuttha@pnl.gov

Chongmin Wang

Senior Research Scientist

(509) 376-4292

chongmin.wang@pnl.gov

Bruce W. Arey

Technologist

(509) 376-3363

bruce.arey@pnl.gov

\section{Key Staff}

Key staff, besides those included above, include Scott A. Chambers, Tim C.

Droubay, Michael A. Henderson, Janos Szanyi, Do Heui Kim, Donald R. Baer, Michael L. Alexander, Weilin Jiang, Yuehe Lin, Todd R. Hart, Jungbae Kim, and Alice Dohnalkova listed. 


\section{Cleanup of Environment Using Iron Nanoparticles}

DR Baer, ${ }^{(a)}$ EJ Bylaska, ${ }^{(a)} \mathrm{KH}$ Pecher, ${ }^{(a)} \mathrm{JE}$ Amonette, ${ }^{(a)} \mathrm{BD}$ Kay, ${ }^{(\mathrm{a})}$ M Dupuis, ${ }^{(a)}$ RL Penn, (b) PG Tratnyek, ${ }^{(c)}$ JT Nurmi, ${ }^{(c)}$ MH Engelhard, (d) and CM Wang(d)

(a) Pacific Northwest National Laboratory, Richland, Washington

(b) University of Minnesota, Minneapolis, Minnesota

(c) Oregon Health Sciences University, Portland, Oregon

(d) W.R. Wiley Environmental Molecular Sciences Laboratory, Richland, Washington

'The use of nanosized particles of iron for cleaning up contaminants in groundwater, soil, and sediments is an exciting new technology contributing to general enthusiasm about nanotechnology. However, there are a lot of unanswered questions about the appropriate and optimal implementation of the nanoiron technology. Researchers from PNNL, in collaboration with Oregon Health Sciences University and the University of Minnesota, have been working during the last few years to understand and exploit the chemical properties of iron nanoparticles. In this research, systematic chemical reaction experiments with nanoparticles have identified some characteristics of nanosized iron particles that may enhance their ability to remove carbon tetrachloride contamination in groundwater.

Carbon tetrachloride is a manufactured toxic chemical historically used in cleaning fluids, as a degreașing agent, and as part of chemical processing. In many locations around the world, industrial waste and spilled liquids have infiltrated the soil and created very large areas of contaminated groundwater and soil.

Our interdisciplinary team includes 10 researchers from PNNL, three from Oregon Health Sciences University, and two from the University of Minnesota. The PNNL researchers have synthesized and characterized several different iron nanoparticles using a variety of advanced microscopy and spectroscopy techniques at EMSL. Chemical reactivity experiments were carried out both at PNNL and Oregon Health and Sciences University using electrochemical techniques.

Several reports indicate that nanosized zero-valent iron ( $\mathrm{Fe} 0$ ) exhibits greater reactivity than micron-sized particles of $\mathrm{Fe} 0$, which would impart advantages for groundwater remediation or other environmental applications. Most of these comparisons were preliminary in that many potentially significant process variables, including the nature of the particles involved, were either uncontrolled or unresolved. To better understand the reactivity of these iron particles, we collected a range of iron nanoparticles with different size and processing history. Because different synthesis, processing, and handling methods produce nanoparticles of various size distributions, with a variety of surface coatings and contaminants, the nanoparticles may have a significant

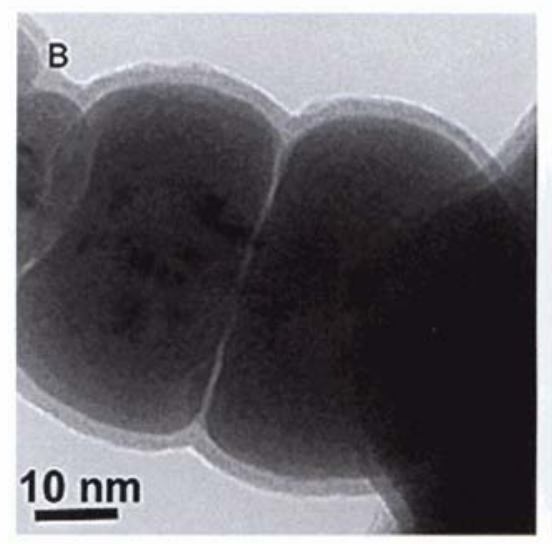

Figure 4-85. HRTEM image showing the nature of the "protecting" layer on a metallic iron nanoparticle. 


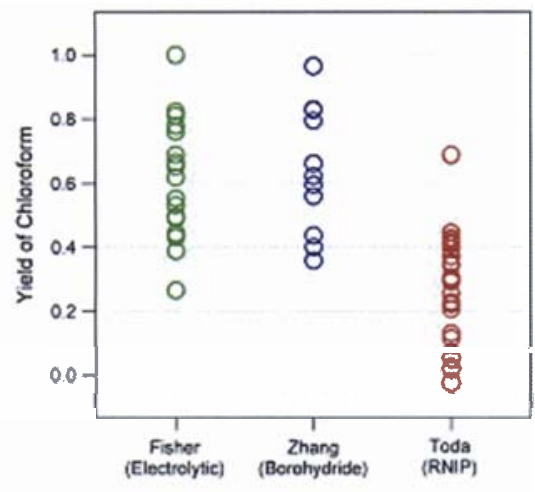

Figure 4-86. Yield of chloroform versus type of iron nanoparticles. Low chloroform yield suggests 2-electron reaction pathway products: $\infty$, etc. Chloroform yield is typically 60 to 80 percent with iron, but one type of nanoiron yields $<0.5$. The yield likely is controlled by the composition of the oxide shell. range of chemical behaviors and reaction properties. Our research focused on measurements of the physical and chemical properties of two materials that have been tested in environmental remediation sites. These iron nanoparticles included single crystal nanoparticles and aggregates of nanoparticles. The particles studied ranged in size from 10 to $100 \mathrm{~nm}$, with the particles coated by an iron-oxide layer of a typical thickness of 2 to $3 \mathrm{~nm}$ or a coating rich in oxidized boron (Figure 4-85) resulting from the synthesis process. Although the reaction rates of the two nanoparticles were similar, the reaction products were significantly different. A commercially produced nanoparticle material that contained both metal particles with a crystalline oxide coating along with fully oxidized nanoparticles degraded carbon tetrachloride to a mixture of relatively harmless products, while larger-sized iron particles and those with boron-rich coatings produced significant amounts of chloroform, an undesirable and toxic by product (Figure 4-86).

This research was included in a special journal issue focused on nanotechnology and the environment. In addition, a February 10,2005, article written by the-' Christian Science Monitor (http://www.csmonitor.com/2005/0210/p14s01-sten. html) was picked up and published by USA Today (http://www.usatoday.com:80/ tech/news/nano/2005-02-10-nano-iron-cleanup x.htm), and related news items have appeared in the Oregonian and the web publication Nanowire.

\section{Citation}

Nurmi JT, PG Tratnyek, V Sarathy, DR Baer,JE Amonette, K Pecher, C Wang, JC Linehan, DW Matson, RL Penn, and MD Driessen. 2005. "Characterization and Properties of Metallic Iron Nanoparticles: Spectroscopy, Electrochemistry, and Kinetics." Environmental Science E̊ Technology 39(5):1221-1230.

\section{Early Stages of Oxidation for CrAlON Nanolayered Coatings Used to Improve Oxidation Resistance of Steel Plates for Applications as Solid-Oxide Fuel Cell Interconnects}

A Kayani, (a) RJ Smith, ${ }^{(a)}$ CV Ramana, (a) PE Gannon, (a) MC Deibert, (a) VI Gorokhovsky, ${ }^{(b)}$ and V Shutthanandan ${ }^{(c)}$

(a) Montana State University, Bozeman, Montana

(b) Arcomac Surface Engineering, LLC, Bozeman, Montana

(c) W.R. Wiley Environmental Molecular Sciences Laboratory, Richland, Washington

Solid-oxide fuel cells are becoming increasingly attractive as a way of converting chemical energy into electrical current by means of the electrochemical 
combination of hydrogen and oxygen via an ion-conducting solid-oxide electrolyte. 'The operational requirements of high-ionic conductivity and good catalytic performance in the fuel cell must be balanced against the practical requirements of low cost and high-temperature corrosion resistance for components in the fuel cell stack. Of particular interest in our work is the bipolar plate serving as the current collector or interconnect between adjacent cells of the solid-oxide fuel cell stack. 'The interconnect must not only retain low electrical resistivity throughout the operating lifetime of the fuel cell, but must also have good surface stability with thermal expansion and other physical properties that are compatible with the materials in the stack.

In our previous work (Smith et al. 2003), we selected the Cr-Al-N multilayer system for study because it not only offers oxidation resistance at temperatures up to $900^{\circ} \mathrm{C}$, but also provides wear resistance typical of many metal nitrides. In our present work, we have selected the Cr-A1-ON system. A variety of nanolayered structures with nanometer-thick layers of alternating chromium oxynitride ( $\mathrm{CrON}$ ) and aluminum oxynitride (A1ON) (Figure 4-87) have been deposited. We expect that by introducing the oxygen during the growth process, the chromium-containing nanolayer will have mixed phases of $\mathrm{Cr}_{2} \mathrm{O}_{3}$ and $\mathrm{CrN}$. Eventually, the loss of nitrogen during the annealing process will lead to the transformation of $\mathrm{CrN}$ to $\mathrm{Cr}_{2} \mathrm{O}_{3}$. We expect that with $\mathrm{Cr}_{2} \mathrm{O}_{3}$ and $\mathrm{Al}_{2} \mathrm{O}_{3}$ already present in the deposited layers, the diffusion associated with additional thermally grown oxides will not affect the layer architecture nearly as much as in the CrAlN coatings of our earlier studies. We used nanolayers of different bilayer thickness consisting of $\mathrm{AlON}$ and $\mathrm{CrON}$ layers, with the goal to study the affect of individual layer thickness on oxidation kinetics for the coating. Sample characterization included ion beam analysis for structure and composition of the coating, atomic force microscopy for surface roughness, and area specific resistance for electrical conductivity.

'Ihe nanolayered coatings were deposited by Arcomac Surface Engineering using the Large-Area Filter Arc Deposition technology with the patented rectangular Large Area Filtered Arc Source ${ }^{\mathrm{m}}$ that overcomes the limitations of conventional filter design. Details about the samples studied here are presented in Table 4.2. Oxidation of the samples was carried out using a standard tube furnace operated horizontally in air, with no additional fixtures to control humidity or flow rate. The oven temperature was controlled electronically with a 30-minute rise time to $800^{\circ} \mathrm{C}$. Soak times were incremented to give total oxidation periods of $1,4,9$, and 25 hrs.

The composition profiles were determined with Rutherford backscattering and nuclear reaction analysis. 'The depth scale $\left(10^{15}\right.$ atoms $\left./ \mathrm{cm}^{2}\right)$ is characteristic of the Rutherford backscattering measurement, which only determines the number of target atoms per $\mathrm{cm}^{2}$ visible to the analysis beam. If the sample density is known, this scale is readily converted to a linear depth scale. Approximately, we can take $1 \times 1015 \mathrm{at} / \mathrm{cm}^{2}$ to be one layer of atoms, or approximately 0.2 $\mathrm{nm}$. 'The coatings analyzed here behave very differently when subjected to the high-temperature oxidizing atmosphere. Sample A 3 survived the oxidation, and after 25 hours of heating the composition remained unchanged, with negligible interdiffusion of heavier elements between the substrate and coating. 


\begin{tabular}{|c|c|c|c|}
\hline Sample & $\begin{array}{c}\text { Sample } \\
\text { Description }\end{array}$ & $\begin{array}{c}\text { 0xygen: } \\
\text { Nitrogen }\end{array}$ & $\begin{array}{c}\text { Approximate Thickness } \\
\mathbf{1 0}^{\mathbf{1 5}} \mathbf{a t} / \mathbf{c m}^{\mathbf{2}}\end{array}$ \\
\hline A3 & CrO/AlO & $1: 0$ & 5150. \\
\hline A9 & CrO/AlO & $1: 0$ & - \\
\hline B3 & CrON/AlON & $2: 3$ & 11650 \\
\hline B9 & CrON/AION & $2: 3$ & 12100 \\
\hline C3 & CrON/AION & $1: 4$ & 13750 \\
\hline C9 & CrON/AION & $1: 4$ & 11950 \\
\hline D3 & CrN/AIN & $0: 1$ & 14950 \\
\hline D9 & CrN/AIN & $0: 1$ & 9550 \\
\hline
\end{tabular}

However, as expected, this sample was not electrically conducting and therefore of limited interest.

Sample B3 was found to be oxidizing as heated, with oxygen gradually replacing nitrogen (Figure 4-88, top row). After 25 hours of heating, less than 5 percent nitrogen is left in the sample. Sample B9, with a thinner bilayer than B3, lost nearly all of the nitrogen after only 1 hour of annealing (Figure 4-88, bottom row). No interdiffusion of elements from substrate to coating was observed in the case of sample B3. For B9, a very small percentage of iron is found diffusing into the coating, but not reaching the surface. This indicates

Table 4-2. Structure Parameters of CrON/AION Coatings on 440A Steels
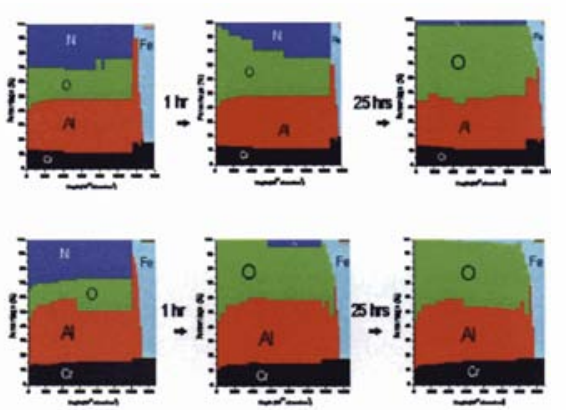

Figure 4-88. Concentration versus depth profiles for the samples B3 (top row) and B9 (bottomrow). that introducing oxygen during the coating growth process creates diffusion barriers that stop or slow down the diffusion of iron from the substrate to the coating. Oxidation of the coatings leads to the formation of $\mathrm{Cr}_{2} \mathrm{O}_{3}$ and $\mathrm{Al}_{2} \mathrm{O}_{3}$, which is evident from the stoichiometry obtained by Rutherford backscattering for these compounds. The most likely mechanism of oxide formation for the nanolayers is the loss of nitrogen from the $\mathrm{CrN}$ as it transforms to $\mathrm{Cr}_{2} \mathrm{~N}$ at the high temperature and the replacement of nitrogen by oxygen in the nanolayers. Results similar to these were reported for high-temperature oxidation of $\mathrm{CrN}$ films on steel. In those experiments, XPS depth profiles show that in addition to the transformation from $\mathrm{CrN}$ to $\mathrm{Cr}_{2} \mathrm{O}_{3}, \mathrm{FeO}$ is forming at the surface as iron diffuses from the steel substrate out through the $\mathrm{CrN}$ coating.

In coating $\mathrm{C} 3$, nitrogen is almost completely lost to oxygen even after only 1 hour of annealing. Also, the sample shows significant iron diffusion from the substrate to the coating surface after only 25 hours in the oven. In coating $\mathrm{C} 9$, the nitrogen loss is almost the same as observed in coating $\mathrm{C} 3$, but the rate at which iron diffusion occurred in $\mathrm{C} 9$ was less than that observed in the case of $\mathrm{C} 3$. In $\mathrm{C}$, iron was not seen on the surface after 25 hours of heating. In coating D3. nitrogen is gradually replaced by oxygen and is completely lost in 25 hours of annealing. Diffusion of iron into the coating occurs even for $\mathbf{1}$ hour of annealing, and after 25 hours of heating iron is found on the surface. Iron diffusion from the substrate to the coating surface in D9 was also observed after only 25 hours in the oven.

In summary, we have measured the initial stages of high-temperature oxidation for 440A stainless steel alloy with nanolayer coatings of $\mathrm{CrON}$ and $\mathrm{AlON}$. The results from ion beam analysis are quantitative and can be used to show either total oxygen content or oxide thickness as a function of time. Ion beam analysis shows that oxidation rates can be greatly reduced with these coatings, and point to a nanolayered structure with sub-nanometer layer thickness as having good performance for periods up to 25 hours at $800^{\circ} \mathrm{C}$ in air. Future measurements will extend the annealing time to better simulate the interconnect application in a solid-oxide fuel cell.

\section{Citation}

RJ Smith, C Tripp, A Knospe, CV Ramana, A Kayani, V Gorokhovsky, V Shutthanandan, and DS Gelles. 2004. "Using CrAlN Multilayer Coatings to Improve Oxidation Resistance of Steel Interconnects for Solid Oxide Fuel Cell Stacks." Journal of Materials and Engineering Performance 13(3):295-302. 


\section{Molecular-Level Processes Governing the Interaction of Contaminants with Iron and Manganese Oxides}

GE Brown, Jr., (a) TJ Kendelewicz, ${ }^{(a)}$ SA Chambers, ${ }^{(b)}$ JE Amonette, ${ }^{(b)}$ JR Rustad, (b) S Thevuthasan, ${ }^{(c)}$ and NS Foster-Mills ${ }^{(c)}$

(a) Stanford University, Palo Alto, California

(b) Pacific Northwest National Laboratory, Richland, Washington

(c) W.R. Wiley Environmental Molecular Sciences Laboratory, Richland, Washington

Starting in December 1996, Gordon Brown and his research group at Stanford University, along with PNNL researcher Scott Chambers, began a collaboration that relied heavily on the preparation of thin-film iron oxides grown by oxygenplasma-assisted molecular beam epitaxy (MBE) on appropriate lattice-matched substrates. Iron oxides are among the most common and important natural sorbents in the Earth's near-surface environment and are responsible for sequestering (and in some cases transforming) many toxic metals and metalloids in groundwater aquifers and soils, including $\mathrm{Cr}$ (VI), $\mathrm{As}$ (III), $\mathrm{Se}(\mathrm{VI}), \mathrm{Cd}(\mathrm{II})$, $\mathrm{Hg}(\mathrm{II})$, and $\mathrm{Pb}$ (II). "The main benefit of employing thin-film MBE-grown iron oxides rather than natural iron oxides is that the composition and surface perfection of the former can be carefully controlled, whereas in the latter, the impurity content and surface imperfections can result in very complex samples that make fundamental studies of reaction mechanisms very difficult due to the number of variables that must be taken into account. Our initial collaboration was funded through a grant from the DOE Environmental Management Science Program to Chambers and Brown, and its main focus was a detailed molecularlevel study of the reduction of the highly toxic and mobile form of chromium $\left[\mathrm{Cr}(\mathrm{VI}) \mathrm{O}_{4}{ }^{2-}\right]$ to the non-toxic and immobile form $\mathrm{Cr}$ (III) on MBE-grown iron oxides containing $\mathrm{Fe}$ (II). Because of the complexity of the interfaces between iron oxides and aqueous solutions, a necessary precursor to this study was a detailed examination of the interaction of water with these surfaces, which was accomplished using synchrotron-based photoemission spectroscopy. This study showed significant differences in reactivity between $\alpha-\mathrm{Al}_{2} \mathrm{O}_{3}(0001)$ and $\boldsymbol{\alpha}-\mathrm{Fe}_{2} \mathrm{O}_{3}(0001)$ with respect to water (Liu et al. 1998; Kendelewicz et al. 2000a),. 'These studies were followed by photoemission and x-ray absorption spectroscopy studies of the interaction of aqueous $\mathrm{Cr}(\mathrm{VI})$ with $\mathrm{Fe}(\mathrm{II})$-containing oxide surfaces, including both $\mathrm{MBE}$-grown single crystal magnetite $\left(\mathrm{Fe}_{3} \mathrm{O}_{4}\right)$ (Kendelewiczet al. 1999) and thin-film a-Fez03 (Grolimund et al. 1999; Brown et al. 2001), in which surface $\mathrm{Fe}$ (III) was partially reduced by heating under ultrahigh vacuum conditions to produce thin selvages of magnetite.

'This work was followed by similar studies of the interaction of aqueous $\mathrm{Cr}(\mathrm{VI})$ with natural magnetite surfaces (Kendelewicz et al. 2000b). These studies of 
(a)

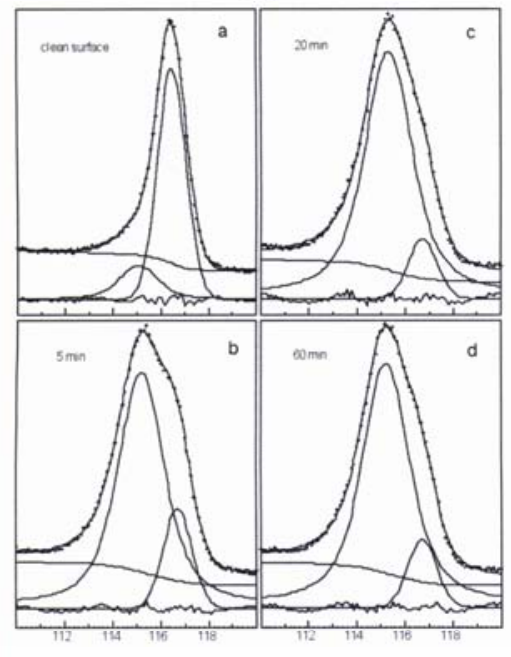

Figure 4-89. (a) Oxygen Is photoemission spectra of a $\mathrm{Cr}(\mathrm{Vl})_{\text {aq- }}$ reacted magnetite (111) sample as a function of dosing time; (b) Oxygen K-edge $\mathrm{x}$-ray absorption spectra of $\mathrm{Cr}(\mathrm{VI})_{\text {aq-}- \text { reacted magnetite(111) as a }}$ function of dosing time (in minutes).

both MBE-grown and natural magnetites clearly showed that $\mathrm{Cr}(\mathrm{VI})$ is rapidly reduced to $\mathrm{Cr}$ (III) by magnetite. However, they also showed that the magnetite surface is quickly passivated by thin layers of $(\mathrm{Fe}, \mathrm{Cr}) \mathrm{OOH}$, which stops the electron transfer reaction when the passivating layer reaches a thickness of 15 to $20 \AA$ (Kendelewicz et al. 1999; Brown et al. 2001). As an example, evidence for extensive hydroxylation in the overlayer was provided by a chemically shifted component in the $\mathrm{O}$ Is photoemission (Figure 4-89a) and $\mathrm{O} \mathrm{K}$-edge absorption

(b)

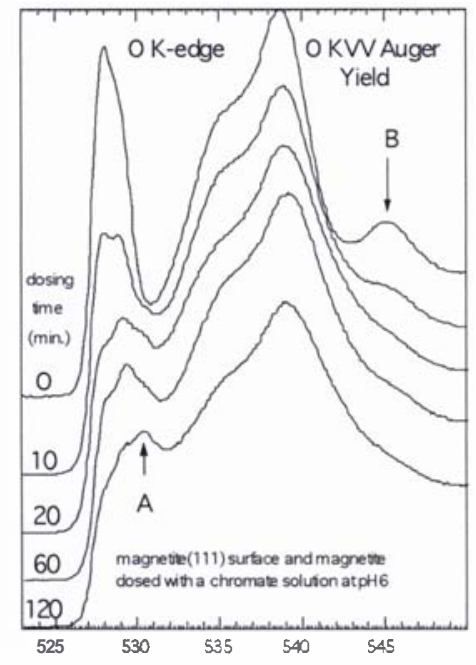

spectra taken with surface-sensitive $\mathrm{O}$ KVV Auger yield (feature A in Figure 4-89b). 'The overlayer appears to lack long-range order based on loss of the first $\mathrm{x}$-ray absorption fine structure-like feature in the $\mathrm{O} \mathrm{K}$-edge spectrum with increasing dosing time (feature B in Figure 4-89b). Clear evidence for oxidation of $\mathrm{Fe}$ (II) to $\mathrm{Fe}$ (III) in the surface region of the magnetite substrate during reduction of $\mathrm{Cr}(\mathrm{VI})_{\mathrm{aq}}$ to $\mathrm{Cr}$ (III) was provided by iron $2 \mathrm{p}$ photoemission and iron L-edge absorption spectra (not shown). Strong attenuation of the iron $2 \mathrm{p}$ signal during the first 10 minutes of the redox reaction indicated that iron does not out-diffuse to the overlayer. "This finding has important implications for the use of magnetite and zero-valent iron particles in reactive permeable barrier remediation technologies to reduce aqueous chromate and remove it from groundwater plumes.

An important adjunct to these studies was a first attempt to determine the surface structure of MBE-grown a-Fez03 (0001) using X-ray photoelectron diffraction (Thevuthasan et al. 1999). 'This initial work on the surface structure and reactivity of hematite surfaces was followed by grazing incidence $\mathrm{x}$-ray absorption fine structure spectroscopy on the interaction of aqueous $\mathrm{Pb}$ (II) with different surfaces [(0001) and (1-102)] of MBE-grown hematite, which showed major differences in the reactivity of these two surfaces with respect to $\mathrm{Pb}$ (II) (Bargar et al. 2004). It also led to X-ray standing wave-fluorescence yield studies of the impact of microbial biofilms (Templeton et al. 1999 and 2001) and thin polymer films (Yoon et al. 2005) on the reactivity of different surfaces of a-Fez03 and a-A1203 with respect to aqueous $\mathrm{Pb}(\mathrm{II})$, and to the first crystal truncation rod diffraction study of the hydrated hematite (0001) (Trainor et al. 2004 and 2003) (1-102) surfaces under in situ conditions (i.e., in the presence of water vapor). 'These crystal truncation rod diffraction studies provided the first detailed structures of hydrated iron-oxide surfaces, which are the relevant surfaces for understanding the interaction of environmental pollutants such as $\mathrm{Cr}(\mathrm{VI})$ and $\mathrm{Pb}(\mathrm{II})$ with iron oxides under environmentally realistic conditions.

In summary, the Chambers-Brown collaboration, built around MBE-grown ironoxide thin films, has resulted in a number of projects and results that have directly impacted pollution problems at the Hanford Site in Richland, Washington, and at other similar sites within the DOE complex. "This collaborative research has also led to a fundamental understanding of the interactions of aqueous metal and metalloid ions at complex environmental interfaces. 
BargarJR, T P Trainor,JP Fitts, SA Chambers, and GE Brown,Jr. 2004. “In-situ Grazing Incidence EXAFS Study of Pb(II) Chemisorption on Hematite (0001) and (1-102)." Langmuir 20(5):1667-1673.

Brown GEJr., SA Chambers, JE Amonette, JR Rustad, T Kendelewicz, P Liu, CS Doyle, D Grolimund, NS Foster-Mills, SA Joyce, and S Thevuthasan. 2001. "Interaction of Water and Aqueous Chromium Ions with Iron Oxide Surfaces." American Chemical Society Symposium Series 778, Nuclear Site Remediation - First Accomplishments of the Environmental Management Science Program. Eds. P.G. Eller and W.R. Heineman, pp. 212-246, American Chemical Society, Columbus, Ohio.

Grolimund D, T Kendelewicz, T P Trainor, P Liu, JP Fitts, SA Chambers, and GE Brown, Jr. 1999. "Identification of Cr Species at the Solution-Hematite Interface after $\mathrm{Cr}(\mathrm{VI})$ - $\mathrm{Cr}$ (III) Reduction using GI-XAFS and Cr L-edge NEXAFS." Journal of Synchrotron Radiation 6(3):612-614.

Liu P, T Kendelewicz, GE Brown, Jr., EJ Nelson, and SA Chambers. 1998.

"Reaction of Water with a-A1203 and $\alpha-\mathrm{Fe}_{2} \mathrm{O}_{3}$ (0001) Surfaces: Synchrotron X-ray Photoemission Studies and Thermodynamic Calculations." Surface Science 417(1), 53-65.

KendelewiczT, P Liu, CS Doyle, GE Brown, Jr., EJ Nelson, and SA Chambers. 2000a. "Reaction of Water with the (100) and (111) Surfaces of $\mathrm{Fe}_{3} \mathrm{O}_{4}$." Surface Science 453(13):32-46.

Kendelewicz T, P Liu, CS Doyle, and GE Brown, Jr. 2000b. "Spectroscopic Study of the Reaction of Aqueous $\mathrm{Cr}(\mathrm{VI})$ with $\mathrm{Fe}_{3} \mathrm{O}_{4}$ (111) Surfaces." Surface Science 469(23):144-163.

KendelewiczT, P Liu, CS Doyle, GE Brown,Jr., EJ Nelson, and SA Chambers. 1999. "X-ray Absorption and Photoemission Study of the Adsorption of Aqueous $\mathrm{Cr}(\mathrm{VI})$ on Single Crystal Hematite and Magnetite Surfaces." Surface Science 424(2-3):219-231.

Templeton AS, T P Trainor, SJ Traina, A M Spormann, and GE Brown, Jr. 2001. " $\mathrm{Pb}(\mathrm{II})$ Distribution at Biofilm-Metal Oxide Interfaces." Proceedings of the National Academy of Sciences. 98(21):11897-11902.

Templeton AS, J D Ostergren, T P Trainor, AL Foster, SJ Traina, A M Spormann, and GE Brown, Jr. 1999. "XAFS and XSW Studies of the Distribution and Chemical Speciation of Pb Sorbed to Biofilms on a-A1203 and a-FeOOH Surfaces." Journal of Synchrotron Radiation 6(3):642-644.

Thevuthasan S, YJ Kim, SI Yi, SA Chambers, J Morais, R Denecke, CS Fadley, P Liu, T Kendelewicz, and GE Brown,Jr. 1999. "Surface Structure of MBE-grown $\alpha-\mathrm{Fe}_{2} \mathrm{O}_{3}(0001)$ by Intermediate-energy X-ray Photoelectron Diffraction." Surface Science 425(2-3):276-286.

Trainor TP, A M Chaka, PJ Eng, M Newville, GA Waychunas, JG Catalano, and GE Brown, Jr. 2004. "Structure and Reactivity of the Hydrated Hematite (0001) Surface." Surface Science 573(2):204-224.

Trainor TP, PJ Eng, JG Catalano, GE Brown, Jr., and GA Waychunas. 2003. " Crystal Truncation Rod Diffraction Study of the Hydrated a-Fez03 (1-102) Surface." 2003 Activity Report, Advanced Photon Source. Argonne National Laboratory, Argonne, Illinois.

Yoon TH, T P Trainor, PJ Eng, JR Bargar, and GE Brown, Jr. 2005. "Trace Element Partitioning at Polymer Film-Metal Oxide Interfaces: Long-period X-ray Standing Wave Study." Langmuir (in press). 


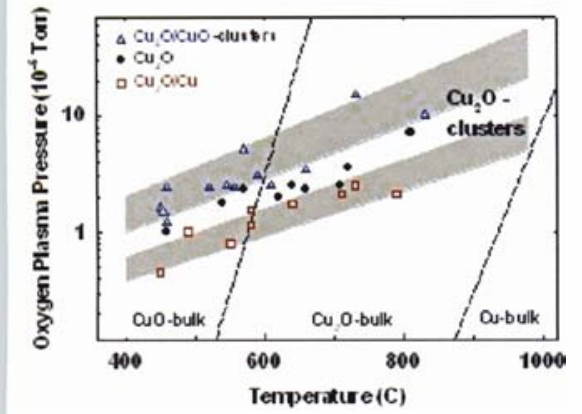

Figure 4-90. Phase diagram for the formation of the $\mathrm{Cu}_{2} \mathrm{O}$ nanodots.

\section{Formation of Epitaxial Oxide Nanodots on Oxide Substrate: $\mathrm{Cu}_{2} \mathbf{O}$ on Pure and Patterned $\mathrm{SrTiO}_{3}(100)$}

DR Baer, ${ }^{(a)}$ Y Liang, ${ }^{(a)}$ A El-Azab, ${ }^{(a)}$ Y Du, ${ }^{(b)}$ J Groves, ${ }^{(b)}$ PNachimuthu, ${ }^{(c)}$ I Lyubinetsky, ${ }^{(d)}$ and S Thevuthasan ${ }^{(d)}$

(a) Pacific Northwest National Laboratory, Richland, Washington

(b) University of Virginia, Charlottesville, Virginia

(c) Lawrence Berkeley National Laboratory, Berkeley, California

(d)W.R. Wiley Environmental Molecular Sciences Laboratory, Richland, Washington

'The lattice mismatched heteroepitaxial growth, driven by interfacial elastic strain, offers a possibility of producing self-assembled coherent (defect-free) quantum dots. Although there has been success in the formation and analysis of semiconductor quantum dots, much less attention has been devoted to oxide quantum dots. Oxides constitute a highly diverse class of materials with rich optical, electronic, magnetic, and dielectric properties, leading to a variety of current and potential technologicalapplications. Combined with their frequent chemical stability in a variety of environments, the technologically relevant properties of oxides are stimulating an increased interest into investigation of the formation and properties of nanoscale oxide structures. Copper oxide, $\mathrm{CuzO}$, is of particular interest because it has a unique electronic structure, suggesting possible catalytical and photocatalytical applications.

Oxygen plasma-assisted MBE has been successfully used to grow $\mathrm{CuzO}$ nanodots on pure $\mathrm{SrTiO}_{3}(100)$ surfaces (Lyubinetskyet al. 2003a, 2003b, and 2004; Du et al. 2004; El-Azab and Liang 2003; Nachimuthu et al. 2003; 'Thevuthasan et al. 2003; Liang et al. 2001). Spontaneous self-assembled island nucleation results from the compressive strain derived from the large lattice mismatch (8.9 percent) between $\mathrm{Cu}_{2} \mathrm{O}$ and $\mathrm{SrTiO}_{3}$. The chemical state of the synthesized nanodots has been verified by combined XPS and Auger electron spectroscopy analysis. Evaluating the experimental parameters for the $\mathrm{CuzO}$ nanodots formation, a phase diagram as a function of growth temperature and oxygen pressure been determined and is shown in Figure 4-90. It is remarkable that at the majority of growth parameters, mixed phases $\left(\mathrm{CuO} / \mathrm{Cu}_{2} \mathrm{O}\right.$ or $\mathrm{Cu}_{2} \mathrm{O}$ / $\mathrm{Cu}$ ) coexist. The region associated with the growth of pure $\mathrm{CuzO}$ nanodots is observed to be very narrow, especially if compared to the bulk $\mathrm{Cu}-\mathrm{O}$ phase diagram, making it quite a challenge to achieve the formation of nanodots containing a single phase of cuprous oxide. "There also are two distinctive mixedphase bands in phase space for the nanodots. It is considerably different from the bulk, that outside of the fine $\mathrm{CuzO}$ region in the phase diagram for the nanodots, there are the bands of temperature and pressure, within which two phase-like forms coexist, not as the rather sharp phase boundaries observed for the bulk. 
The initial stages of the $\mathrm{Cu}_{2} \mathrm{O}$ island growth and interface formation have been examined by XPS. An absence of the continuous (wetting) layer formation has been found, with the metal oxide clusters starting to grow already at submonolayer coverages. XPS and the near edge x-ray absorption fine structure spectroscopy observations indicate some additional interaction between deposited copper atoms and oxygen of the SrTiOs substrate. Atomic force microscopy examination shows formation of isolated square-based, truncated small nanodots at very initial stages of growth (Figure 4-91a). Both the shape and orientation of nanodots, with its edges mostly along the $<011>$ direction, indicate a crystalline ordering and epitaxy, which has been confirmed by XRD data. Lateral dimensions of nanodots do not change significantly in the process of the initial quasi-planar growth (but increase exponentially with temperature). As a result, a dense layer of small, low aspect ratio nanodots forms at this stage of growth. In addition to the small nanodot layer, a subpopulation of considerably larger clusters also appears (Figure 4-91b). Large nanodot density increases when growth proceeds to larger thickness. It is remarkable that these large dots start to form only when the small nanodots reach some critical density, as schematicallyshown in Figure 4-91c. 'The theoretical simulation, based on the kinetic model of the surface morphological evolution, have showed that dot coalescence, driven by increasing dot density, decreasing separation, and fluctuations in dot size and separation during the grown/formation process, is the mechanism responsible for the sudden appearance of large dots superposed on the distribution of small ones.

Regarding the growth on patterned $\mathrm{SrTiO}_{3}$ surfaces, we have synthesized $\mathrm{Cu}_{2} \mathrm{O}$ quantum dots on $\mathrm{SrTiO}_{3}$ substrates, employing a high-vacuum oxygen plasma-assisted MBE system at PNNL for quantum dot growth on surfaces patterned using the University of Virginia's focused ion beam facility (Figure 4-92). Atomic force microscopy of the STO substrates before $\mathrm{Cu}_{2} \mathrm{O}$ deposition (Figure 4-92a) reveals that focused ion beam-induced topography evolves into square-sided pits during pre-growth substrate preparation (wet etch and anneal). Subsequent $\mathrm{Cu}_{2} \mathrm{O}$ deposition generates a regular array of auantum dots (Figure 4-92b).

In summary, formation of pure crystalline $\mathrm{Cu}_{2} \mathrm{O}$ nanodots occurs rather in a narrow growth parameter window, outside which a coexistence of the multiple phases has been observed. Cuprous oxide nanodots on the $\mathrm{SrTiO}_{3}(100)$ substrate follow a growth mechanism, which differs significantly from the growth modes observed for the majority of semiconductor quantum dots. Growth starts without wetting layer formation with the appearance of well-ordered truncated square-based nanodots at submonolayer coverages. At the initial stages of growth, the nanodot size only weakly changes with coverage and exponentially scales with temperature. After reaching a critical, temperature-dependent dot density, growth of mid-sized nanoclusters starts through coalescence. The coexistence of the different types of the clusters at high coverages results in a multimodal distribution of sizes and shapes. Furthermore, focused ion beampatterned SrTiOs substrates have been successively used to control the $\mathrm{Cu}_{2} \mathrm{O}$ nanodot growth location.
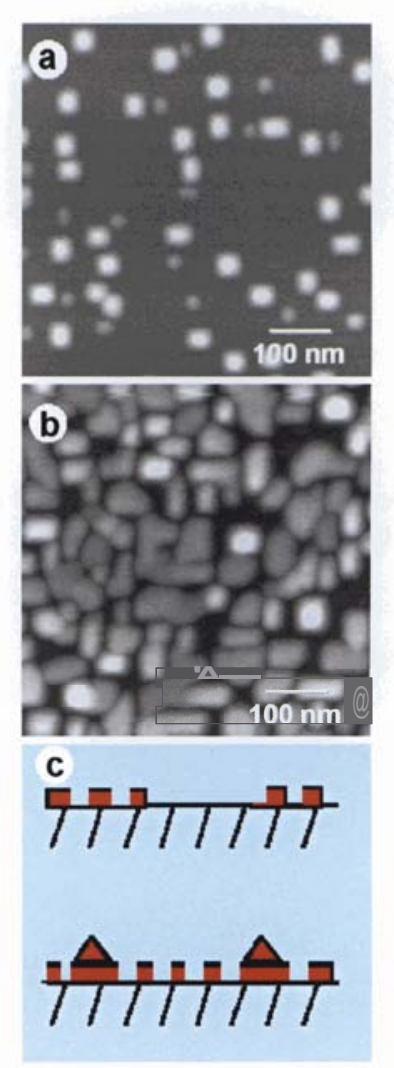

Figure 4-91. Atomic force microscopy images of the surface morphology evolution at temperature of $990 \mathrm{~K}:$ (a) $\mathrm{d}=$ $1 \mathrm{~nm}$, (b) $\mathrm{d}=3 \mathrm{~nm}$. (c) Growth schematics. 


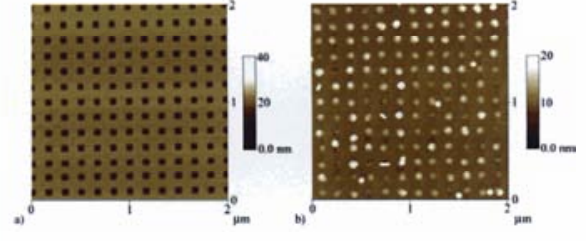

Figure 4-92. a) STO (100) after focused ion beam patterning, etch, and anneal, b) after 0.7 $\mathrm{nm} \mathrm{Cu}_{2} \mathrm{O}$ deposition $(-3000$ $\mathrm{Ga}^{+}$ions $/$spot, $1000^{\circ} \mathrm{C} / 30 \mathrm{~min}$. anneal, $700^{\circ} \mathrm{C} \mathrm{Cu}_{2} \mathrm{O}$ growth, $1 \times 10^{-5}$ Torr oxygen pressure).
Citations

Du Y, S Atha, R Hull, JF Groves, I Lyubinetsky, and DR Baer. 2004. "Focused-IonBeam-Directed Self-Assembly of $\mathrm{Cu}_{2} \mathrm{O}$ Islands on $\mathrm{SrTiO}_{3}(100)$." Applied Physics Letters 84(25):5213-5215.

El-Azab A, and Y Liang. 2003. "Nanoscale Copper Oxide Ring Structure on an $\mathrm{SrTiO}_{3}$ Substrate." Philosophical Magazine 83(31-34):3847-3869.

Liang Y, AS Lea, DE McCready, and P Meethunkji. 2001. State-of-the-ArtApplication of Surface and Interface Analysis Methods to Environmental Material Interactions: In Honor of James E. Castle's 65th Year. Eds. DR Baer, CR Clayton, GP Halada, and G D Davis, pp. 125, Electrochemical Society, Washington, DC.

Lyubinetsky I, A El-Azab, AS Lea, S 'Ihevuthasan, and DR Baer. 2004. "Initial Stages of Oxide Nanodot Heteroepitaxial Growth: $\mathrm{Cu}_{2} \mathrm{O}$ on $\mathrm{SrTiO}_{3}(100)$." Applied Physics Letters 85(19):4481-4483.

Lyubinetsky I, S 'Ihevuthasan, DE McCready, and DR Baer. 2003a. "Formation of Single-Phase Oxide Nano-Clusters: $\mathrm{Cu}_{2} \mathrm{O}$ on $\mathrm{SrTiO}_{3}(100)$." Journal of Applied Physics 94(12):7926-7928.

Lyubinetsky I, S Thevuthasan, DE McCready, AS Lea, and DR Baer. 2003b. "Formation of Self-Assembled Cuprous Oxide Nano-Dots on $\mathrm{SrTiO}_{3}(100)$ Surfaces." In Proceedings of the Institute of Electrical and Electronics Engineers Conference on Nanotechnology 2003, San Francisco, California.

Nachimuthu P, S 'Ihevuthasan, YJ Kim, AS Lea, V Shutthanandan, MH Engelhard, DR Baer, SA Chambers, DK Shuh, D W Lindle, EM Gullikson, and RCC Perera. 2003. "Investigation of Copper(I) Oxide Quantum Dots by Near Edge X-ray Absorption Fine Structure Spectroscopy." Chemistry of Materials 15(20):3939-3946.

'Ihevuthasan S, P Nachimuthu, YJ Kim, I Lyubinetsky, AS Lea, V Shutthanandan, M H Engelhard, DR Baer, DK Shuh, and DW Linde. 2003. "Surface and Interface Characterization of Self-Assembled Copper Oxide Quantum Dots on $\mathrm{SrTiO}_{3}(001)$ Surface." Proceedings of Nanotech 20033 (2003) 278.

\section{Nanostrucutures for Enzyme Structures}

JB Kim, (a) JH Kwak, (a) JW Grate, ${ }^{\left({ }^{(a)}\right.}$ A S Lea, (b) CM Wang, (b) J Lee, ${ }^{\text {(c) }}$ CW Lee, ${ }^{(c)} \mathrm{KS} \mathrm{Min,}^{(\mathrm{c})} \mathrm{H} \mathrm{B} \mathrm{Na,}^{(\mathrm{c})} \mathrm{H} \mathrm{K} \mathrm{Ahn,}{ }^{(\mathrm{d})}$ and BS Kim${ }^{(\mathrm{d})}$

(a) Pacific Northwest National Laboratory, Richland, Washington

(b) W.R. Wiley Environmental Molecular Sciences Laboratory, Richland, Washington

(c) Seoul National University, Seoul, South Korea

(d)Chungbuk National University, Cheongju, South Korea

Enzymes are useful biocatalysts of nanometer scale that regulate the chemistry of cells and organisms. 'The potential application of enzymes as practical biocatalysts is well recognized and also growing. The unique activities and specificities of 
enzymes play a key role in yielding the wide range of applications. However, the widespread application of enzymes is generally limited by their short lifetimes. We have developed various nanostructures that can be used to stabilize enzyme activity for several applications.

'Ihe first unique nanostructure is "single enzyme nanoparticles." We have developed a new nanostructure containing an enzyme within a hybrid organic/ inorganic polymer network with sufficient porosity to allow substrates to diffuse to the active site (Kim and Grate 2003). 'The synthetic procedure, entailing enzyme modification and two orthogonal polymerization steps, yields nanoparticles containing a single enzyme that can be observed by TEM (Figure 4-93). In experiments with $\alpha$ chymotrypsin, incorporation into the nanostructure

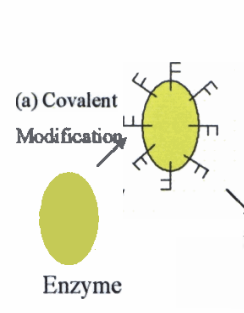
dramatically increased enzymatic stability.

Furthermore. the nanoscale structure around the enzyme is sufficiently thin that it does not impose a significant mass transfer limitation on the substrate. Because these nanoparticles remain soluble or suspended in solutions, they can be processed into a variety of forms.

'Ihe second nanostructure is mesoporous media (Figure 4-94). W e have developed nanoscale enzyme reactors in mesoporous silica via a ship-in-abottle approach, which employs adsorption of enzymes followed by crosslinking using glutaraldehyde treatment (Lee et al. 2004). Mesocellular mesoporous silica, small particles (200 to $500 \mathrm{~nm}$ ) with large mesocellular pores $(37 \mathrm{~nm})$ connected by mesoporous channels $(13 \mathrm{~nm})$, was selected as a carrier of nanoscale enzyme reactors. The resulting nanoscale enzyme reactors show an impressive stability and activity with an extremely high loading of enzymes. For example, nanoscale enzyme reactors containing a-chymotrypsin could hold $0.5 \mathrm{~g}$ of chymotrypsin in $1 \mathrm{~g}$ of silica, which is comparable to the maximal loading of chymotrypsin in mesocelluar mesporous silica. However, the specific activity of nanoscale enzyme reactors containing $\boldsymbol{\alpha}$-chymotrypsin was 4.2 times higher than that of the adsorbed chymotrypsin, with a lower loading ( $0.07 \mathrm{~g}$ of chymotrypsin per $1 \mathrm{~g}$ of silica), which was further decreased by a continuous leaching of adsorbed chymotrypsin.

'Ihe final nanostructure is electrospun nanofibers. Nanofibers consisting of enzyme-polymer composites have been directly prepared (Figure 4-95) by electrospinning a toluene solution containing surfactant-stabilizedenzyme and polymer (Kim et al. 2005). Additional treatment with glutaraldehyde could greatly stabilize the enzyme activity of the fibers, which could be maintained in a buffer under shaking conditions for more than two weeks. 'The nanofibers also showed great improvement in the enzyme activity over bulk films as a result of increased mass-transfer for substrate molecules to and from the enzyme reactive sites. 'The apparent specific activity of nanofibers with an enzyme loading of 6.3 percent could be achieved up to 40 percent of that of free enzymes. "These stable and catalytically active nanofiber-based mats were highly durable and could be easily recovered from a solution, making them ideal candidates for large-scale applications. We have also developed a unique approach for the fabrication of
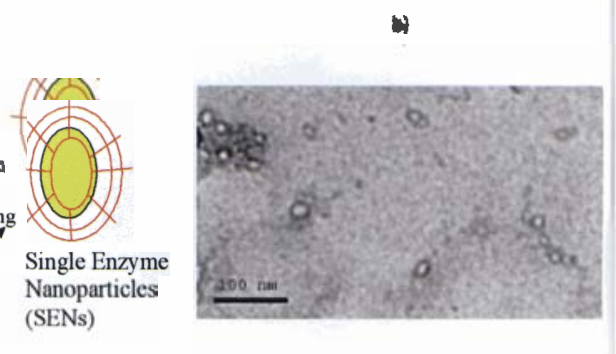

Figure 4-93. Schematic of single enzyme nanoparticlesynthesis (a) and transmission electron microscopy images SEN-CT (b)

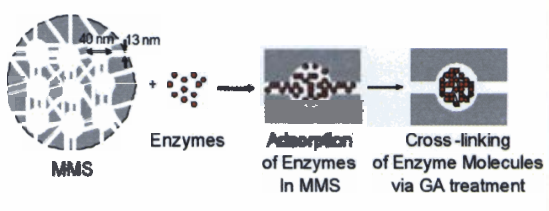

Figure 4-94. Schematic diagram for nanometer-scale enzyme reactors mesocellular mesoporous silica. 


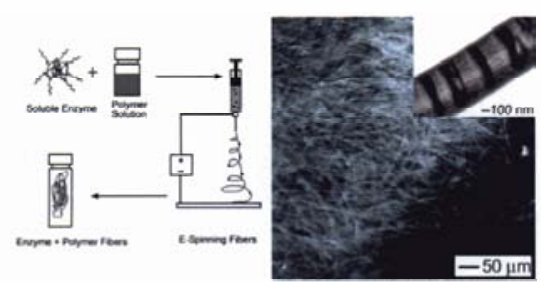

Figure 4-95. Schematic diagram for the preparation of enzymepolymer composite nanofibers. enzyme aggregate coatings on the surfaces of electrospun polymer nanofibers. This approach employs covalent attachment of seed enzymes onto nanofibers consisting of a mixture of polystyrene and poly(styrene-co-maleic) anhydride, followed by a glutaraldehyde treatment that cross-links additional enzyme molecules and aggregates from solution onto the covalently-attached seed enzyme molecules. These cross-linked enzyme aggregates, covalently attached to the nanofibers via the linkers of seed enzyme molecules, are expected to improve the enzyme activity due to increased enzyme loading, and also the enzyme stability.

\section{Citations}

Kim JB, J H Kwak, and JW Grate. 2005. "Direct Fabrication of Enzyme-Carrying Polymer Nanofibers by Electrospinning."Journal of Materials Chemistry (submitted).

Kim JB, and JW Grate. 2003. "Single-Enzyme Nanoparticles Armored by a Nanometer-Scale Organic/Inorganic Network." Nano Letters, 3(9):1219-1222.

Lee J, JB Kim,JH Kwak, and JW Grate. 2004. "Simple Synthesis of Hierarchically Ordered Mesocellular Mesoporous Silica Materials and their Successful Application as a Host of Enzyme Immobilization."Journal of the American Chemical Society (in press).

\section{Synthesis and Characterization of Ferromagnetic Materials}

KA Griffin, (a) A B Pakhomov, (a) P Blomqvist, (a) H Ohldag, ${ }^{\left({ }^{(a)}\right.}$ KM Krishnan, (a) SM Heald, ${ }^{(b)}$ CM Wang, ${ }^{(c)}$ DE McCready, ${ }^{(c)}$ V Shutthanandan, ${ }^{(c)}$ and S Thevuthasan (c)

(a) University of Washignton, Seattle, Washington

(b) Pacific Northwest National Laboratory, Richland, Washington

(c) W.R. Wiley Environmental Molecular Sciences Laboratory, Richland, Washington

Using complementary experiments, we have shown that the room-temperature ferromagnetism observed in anatase $\mathrm{Co}: \mathrm{TiO}_{2}$ films is not carrier mediated, but coexists with the dielectric state (Griffin et al. 2005). TEM and x-ray absorption spectroscopy (Figure 4-96) reveal a solid solution of cobalt in anatase, where cobalt is not metallic but in the +2 state substituting for titanium. Measurements at $300 \mathrm{~K}$ yield a $M_{\mathrm{S}}$ of $1.1 \mu_{\mathrm{B}} /$ cobalt atom, while all films are highly insulating. The evidence of intrinsic ferromagnetism in the dielectric ground state of Co: $\mathrm{TiO}_{2}$ leads to new considerations for the origin of ferromagnetism in transition metal-doped oxides. In fact, we propose that these are a new class of materials: dilute magnetic dielectrics. These results have significant implication for the development of an oxide room-temperature spin electronics device.

In related work, magnetic reversal in ferromagnetic materials occurs either by coherent rotation or by domain nucleation. Generally, the mechanism is an intrinsic function of the microstructure and is symmetric with respect to the applied field. In other words, on either branches of the hysterisis loop only 
one (and the same) of these two mechanisms is observed. We have shown, for the first time, direct imaging evidence for the asymmetry in magnetic reversal mechanisms in exchange-biased systems that is beyond the scope of indirect scattering measurements (Figure 4-97). This was made possible by detailed x-ray photoemission electron microscopy imaging at the Advanced Light Source on carefully prepared samples (Blomqvistet al. 2005 and 2004) which had been previously well-characterized magnetically, including neutron reflectivity measurements at the intense pulsed neutron source at Argonne National Laboratory. 'The magnetization reversal occurs by moment rotation for decreasing fields, while it proceeds by domain nucleation and growth for increasing fields. The observed domains are consistent with the crystallography of the bilayers and favor a configuration that minimizes the overall magnetostatic energy of the ferromagnetic layer.

Citations

Blomqvist P, MK Kannan, and H Ohldag. 2005. "Direct Imaging of Asymmetric Magnetization Reversal in Exchange-biased Fe/MnPd Bilayers by X-ray Photoemission Electron Microscopy." Physical Review Letters 94(10):107203, 1-4.

Blomqvist P, KM Krishnan, and DE McCready. 2004. "Growth of Exchange-biased $\mathrm{MnPd} / \mathrm{Fe}$ Bilayers." Journal of Applied Physics 95(12):8019-8022.

Griffin KA, AB Pakhomov, CM Wang, SM Heald, and KM Krishnan. 2005. "Intrinsic Ferromagnetism in Insulating Cobalt Doped Anatase $\mathrm{TiO}_{2}$." Physical Review Letters (in press).

\section{Composition and Sourcing of Aerosol in the Mexico City Metropolitan Area with PIXE/PESA/STIM and Multivariate Analysis}

KS Johnson, ${ }^{(a)}$ B Zuberi, ${ }^{(a)}$ B de Foy, ${ }^{(a)}$ LT Molina, ${ }^{(a)}$ MJ Molina, ${ }^{(a)}$ V. Shutthanandan, ${ }^{(b)}$ Y Xie, ${ }^{(b)}$ R Disselkamp, ${ }^{\left({ }^{b}\right)} K_{\text {Dzepina, }}{ }^{(c)} \mathrm{J}$ Jimenez, ${ }^{(c)}$ and D Salcedo ${ }^{(\mathrm{d})}$

(a) Massachusetts Institute of Technology, Cambridge, Massachusetts

(b)W.R. Wiley Environmental Molecular Sciences Laboratory, Richland, Washington

(c) University of Colorado-Boulder, Boulder, Colorado

(d) Universidad Autónoma del Estado de Morelos, Morelos, México

Air pollution in the Mexico City Metropolitan Area is a serious problem affecting the health of its nearly 20 million habitants (Molina 2002). 'The dangers associated with exposure to particulate matter $\leq 2.5 \mu \mathrm{m}$ in diameter $\left(\mathrm{PM}_{2.5}\right)$ (Borja-Aburto 1998) have demonstrated an immediate need to better understand chemical composition and particulate emissions sources. A three-

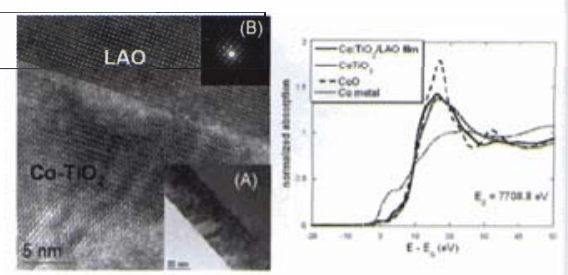

Figure 4-96. Transmission electron micrographand cobalt K-edge x-ray absorption spectra from cobalt-doped $\mathrm{TiO}_{2}$ films grown on $\mathrm{LaAl}_{2} \mathrm{O}_{3}(100)$.

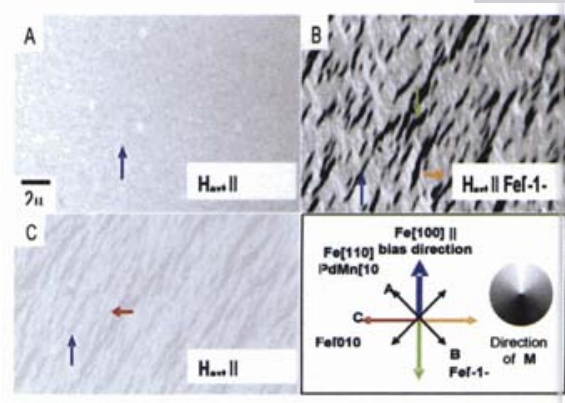

Figure 4-97. Photo-electron emission microscopeimages of the sample saturatedina magnetic field of 1500 De along $\mathrm{Fe}[110]$ (a), $\mathrm{Fe}[-1-10]$ (b), Fe[010] (c), and then measured in zero field. The arrows indicate the direction of the magnetizationin the domains. The bottom panel shows the relationship between the in-plane crystallographic directions, the bias direction, and the direction of saturation, while the grey scale links the direction of the magnetization $(\mathrm{M})$ to the image intensity. 


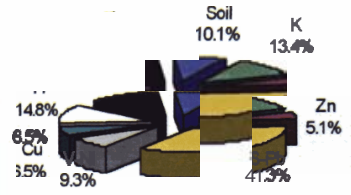

Stage A

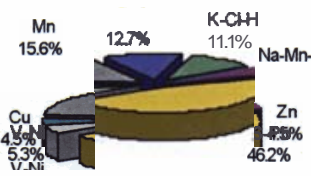

Stage B

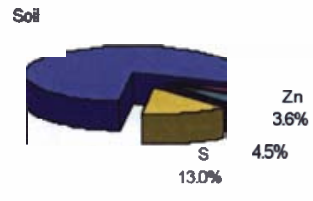

Stage C stage rotating DRUM impactor was used to collect particles in size ranges 1.15 to $2.5 \mu \mathrm{m}$ (Stage A), 0.34 to $1.15 \mu \mathrm{m}$ (Stage B), and 0.07 to 0.34 pm (Stage C) from April 3 to May 4,2003, during the Mexico City Metropolitan Area2003 Field Campaign. Samples were analyzed
Figure 4-98. Positive Matrix Factorization factors of $\mathrm{PM}_{2.5}$ during Mexico City Metropolitan Area-2003 $(A=1.15$ to $2.5 \mu \mathrm{m}, B=0.34$ to $1.15 \mu \mathrm{m}, \mathrm{C}=0.07$ to $0.34 \mu \mathrm{m})$.

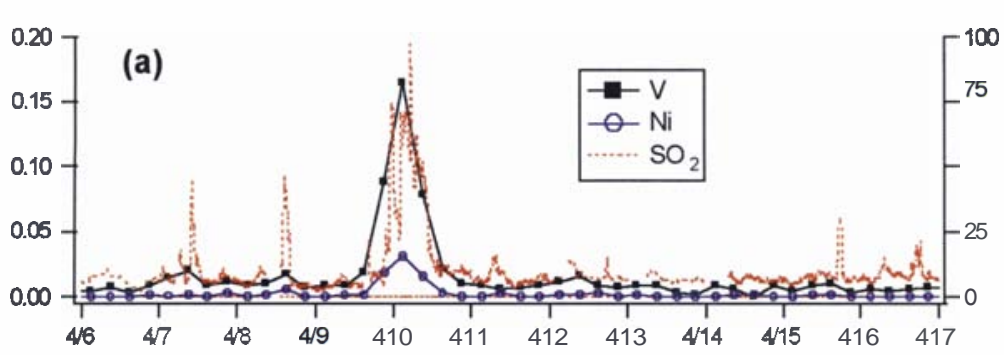

Figure 4-99. Evidence for anthropogenic source of $\mathrm{SO}_{2}$. (a) Time series for vanadium, nickel, and $\mathrm{SO}_{2}$ at CENICA (Mexico City Metropolitan Area2003 sampling site). at PNNL by roto on-induced $\mathrm{x}$-rav emission. proton-elastic scattering analysis, and scanning transmission ion microscopy to determine concentrations of elements sodium-lead, hydrogen, and total ion mass, respectively.

Scanning transmission ion microscopy measured $20.0 \mu \mathrm{g} / \mathrm{m}^{3}$ on average for $\mathrm{PM}_{2.5}$ (sum of all size categories), including all mass except volatile organics, nitrates, and water that were assumed to have been lost during analysis. Among individual proton-induced x-ray emission elements, sulfur $\left(1.74 \mathrm{ug} / \mathrm{m}^{3}\right)$, lead

$\left(0.54 \mathrm{ug} / \mathrm{m}^{3}\right)$, silicon $\left(0.47 \mathrm{ug} / \mathrm{m}^{3}\right)$, and potassium $\left(0.27 \mathrm{ug} / \mathrm{m}^{3}\right)$ were highest in concentration. Positive Matrix Factorization was used to help identify correlations between individual elements to determine possible emissions sources (Figure 4-98).

A silicon-lead factor was dominant in the accumulation mode ( 0.34 to $2.5 \mu \mathrm{m}$, Stages A and B), and most likely related to traffic and secondary sulfate sources. By contrast, silicon and other soil elements (aluminum, californium, magnesium, titanium, manganese, and iron) contributed to the majority of fine aerosol ( 0.07 to $0.34 \mathrm{pm}$, Stage C). Trace metals associated with industrial emissions, and biomass burning markers (potassium, chlorine, and hydrogen) suggest additional emissions sources. Industrial emissions events were observed during Mexico City Metropolitan Area-2003 as sharp increases in trace metals. On April 10, for example, fuel oil components vanadium and nickel correlated with a strong $\mathrm{SO}_{2}$ plume from the north (Figure 4-99), in the direction of a major refinery. 'Thus, an anthropogenic rather than volcanic source of $\mathrm{SO}_{2}$ was most likely responsible; the Popocatepetl volcano lies to the southeast.

Organic carbon may comprise a large fraction of urban aerosol, yet cannot be directly measured by the methods used in this study. However, hydrogen was able to provide a proxy for organic matter after subtracting the contribution to ammonium sulfate (S/4 by molar ratio). Organic hydrogen was in fact nearly equal to sulfate hydrogen during Mexico City Metropolitan Area-2003 (Figure 4-100). Quantitative comparison to organic aerosol measured by aerosol mass spectrometry and determination of percentage lost during low-pressure protonelastic scattering analysis provides an indication of the volatility of organics. In addition, good agreement between the two techniques has been observed for aerosol mass spectrometry sulfate and proton-induced $\mathrm{x}$-ray emission sulfur sulfate equivalent. 
Borja-Aburto VH, M Castillejos, DR Gold, S Bierzwinski, and D Loomis. 1998. Mortality and Ambient Fine Particles in Southwest Mexico City, 1993-1995." Environmental Health Perspectives 106(12):849-855.

LT Molina (ed.). 2002. Air Quality in the Mexico Megacity: An Integrated Assessment. Pp. 408, Kluwer Academic.

Figure 4-100.

Time series for sulfate and organic hydrogen (proton-elastic scattering analysis) and aerosol mass spectrometry organics.

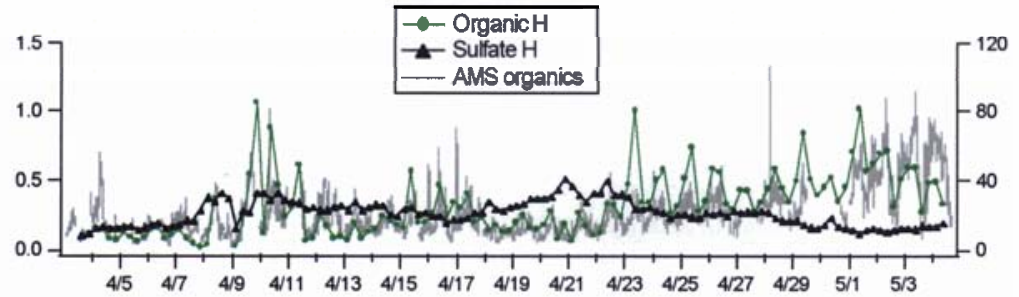

\section{Development of Highly Active Metal Phosphide Hydrodesulfurization Catalysts}

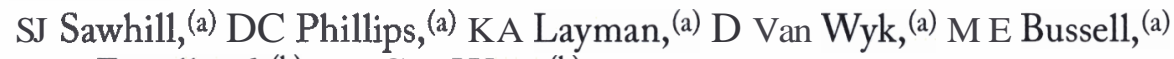
MH Engelhard, ${ }^{(b)}$ and CM Wang ${ }^{(b)}$

(a) Western Washington University, Bellingham, Washington

(b) W.R. Wiley Environmental Molecular Sciences Laboratory, Richland, Washington

Hydrotreating catalysis is a critical process in the petroleum industry, selectively removing sulfur and nitrogen from heteroatom compounds found in crude oil. A new class of materials, the transition metal phosphides, has recently been shown to have high hydrodesulfurization activity and, therefore, may help meet future environmental regulations requiring significant reductions in the allowable sulfur levels in transportation fuels. Studies in our laboratory have investigated the hydrodesulfurization catalytic properties of a number of silicasupported metal phosphide phases; the thiophene hydrodesulfurization activities of these materials are plotted in Figure 4-101. As indicated in the figure, silicasupported molybdenum $\left(\mathrm{MoP} / \mathrm{SiO}_{2}\right)$ and nickel phosphide $\left.\left(\mathrm{Ni}_{2} \mathrm{P} / \mathrm{SiO}\right)_{2}\right)$ have higher hydrodesulfurization activities than conventional sulfide-based catalysts (Phillips et al. 2002; Sawhill et al. 2003 and 2005). Under more industrially relevant hydrotreating conditions, Oyama and coworkers have shown $\mathrm{Ni}_{2} \mathrm{P} / \mathrm{SiO}_{2}$ catalysts to have higher hydrodesulfurization activities than commercial $\mathrm{Ni}$ $\mathrm{Mo} / \mathrm{Al}_{2} \mathrm{O}_{3}$ and $\mathrm{Co}-\mathrm{Mo} / \mathrm{Al}_{2} \mathrm{O}_{3}$ catalysts for the hydrodesulfurization of liquid feeds containing dibenzothiophene and methyl-substituted dibenzothiophenes (Oyama 2003).

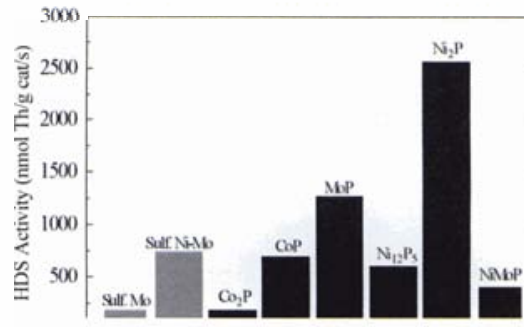

Figure 4-101. Thiophene hydrodesulfurization activities (after $100 \mathrm{~h}$ on-stream) for silicasupported metal phosphide catalysts ( 20 to 25 wt\% MexPy) as well as'for sulfided $\mathrm{Mo} / \mathrm{SiO}_{2}$ $\left(30.4 \mathrm{wt} \% \mathrm{MoO}_{3}\right)$ and $\mathrm{Ni}-\mathrm{Mo} / \mathrm{SiO}_{2}$ catalysts $(7.9 \mathrm{wt} \% \mathrm{NiO}, 30.4 \mathrm{wt} \%$ $\left.\mathrm{MoO}_{3}, \mathrm{Ni} / \mathrm{Mo}=0.5\right)$. 


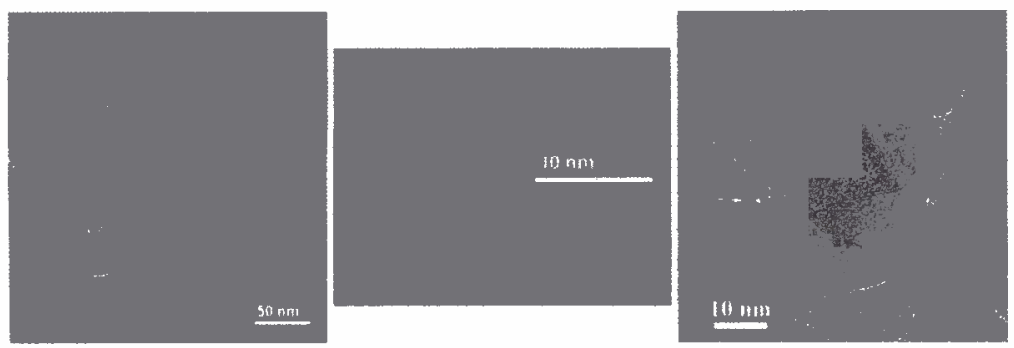

We have undertaken extensive characterization studies of $\mathrm{Ni}_{2} \mathrm{P} / \mathrm{SiO}_{2}$ catalysts, some of which are carried out at EMSL, in order to understand the promising hydrodesulfurization catalytic properties of these materials (Sawhill 2003; Layman and Bussell 2004a). Shown in Figure 4-102 are low- and high-resolution transmission electron micrographs acquired at EMSL of a 25

Figure 4-102. Transmission electron microscopy images of a $25 \mathrm{wt} \% \mathrm{Ni}_{2} \mathrm{P} / \mathrm{SiO}_{2}$ catalyst.

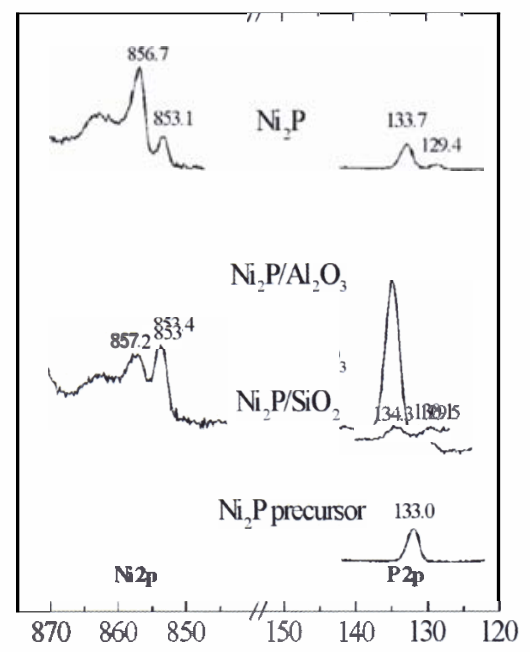

Figure 4-103. XPS spectra for $25 \mathrm{wt} \% \mathrm{Ni}_{2} \mathrm{P} / \mathrm{SiO}_{2}$ and $20 \mathrm{wt} \%$ $\mathrm{Ni}_{2} \mathrm{P}_{\mathrm{Al}} \mathrm{O}_{2} \mathrm{O}_{3}$ catalysts, as well as for unsupported $\mathrm{Ni}_{2} \mathrm{P}$ and its calcined precursor.
The low-resolution image (Figure 4-102, left) shows the silica-supported $\mathrm{Ni}_{2} \mathrm{P}$ to be relatively evenly dispersed on the silica support with particle sizes ranging from 5 to $30 \mathrm{~nm}$. The high-resolution image in Figure 4-102b (middle) reveals that $\mathrm{Ni}_{2} \mathrm{P}$ particles adopt a globular morphology on the silica support, and the thin passivation layer $(-2 \mathrm{~nm})$ formed on the $\mathrm{Ni}_{2} \mathrm{P}$ particles following synthesis is resolved in Figure 4-102c (right). On the basis of infrared spectroscopy and chemisorption measurements, we have concluded that the high hydrodesulfurization activity of $\mathrm{Ni}_{2} \mathrm{P} / \mathrm{SiO}_{2}$ catalysts is associated with both a high site density and a high turnover frequency (Sawhill et al. 2003; Layman and Bussell 2004a and 2004b).

XPS measurements carried out at EMSL indicate the presence of oxidized nickel $\left(853.5 \mathrm{eV}, \mathrm{Ni}^{2}+\right.$ ) and phosphorus $\left(134.3 \mathrm{eV}, \mathrm{PO}_{4}{ }^{3-}\right)$ species in the surface passivation layer of a $\mathrm{Ni}_{2} \mathrm{P} / \mathrm{SiO}_{2}$ catalyst, as well as reduced nickel (853.5 $\mathrm{eV}, \mathrm{Ni}^{\delta^{+}}$and phosphorus (129.5 eV, $\mathrm{P}^{\delta}$ ) in the underlying $\mathrm{Ni}_{2} \mathrm{P}$ particles (see Figure 4-103). Comparison of the binding energies with literature spectra indicates a small amount of electron transfer from nickel to phosphorus in $\mathrm{Ni}_{2} \mathrm{P}$, but the magnitude of $\delta$ is small. "This conclusion is consistent with DFT calculations carried out by Rodriguez et al. 2003, which show the extent of charge transfer to be small. Based upon the XPS and other characterization results, we conclude that the high turnover frequency of silica-supported $\mathrm{Ni}_{2} \mathrm{P}$ is associated with the low-oxidation state of nickel, the participation of surface phosphorus in bonding with adsorbed species, and the strong resistance of $\mathrm{Ni}_{2} \mathrm{P}$ toward sulfur incorporation under the sulfiding conditions found in a hydrodesulfurization reactor.

Since commercial hydrotreating catalysts are typically supported on $\gamma-\mathrm{Al}_{2} \mathrm{O}_{3}$, we have also investigated the hydrodesulfurization properties of alumina-supported $\mathrm{Ni}_{2} \mathrm{P}$. While a small amount of excess phosphorus $(\mathrm{P} / \mathrm{Ni}=0.8)$ was necessary to prepare phase pure $\mathrm{Ni}_{2} \mathrm{P}$ on silica, a large excess of phosphorus $(\mathrm{P} / \mathrm{Ni}=2.0)$ was required to synthesize phase-pure $\mathrm{Ni}_{2} \mathrm{P}$ on $\gamma-\mathrm{Al}_{2} \mathrm{O}_{3}$. Examination of the XPS spectrum of the $\mathrm{Ni}_{2} \mathrm{P} / \mathrm{Al}_{2} \mathrm{O}_{3}$ catalyst in Figure 4-103 reveals the peak intensities and binding energies in the $\mathrm{Ni}(2 \mathrm{p})$ region to be similar to those of the $\mathrm{Ni}_{2} \mathrm{P} / \mathrm{SiO}_{2}$ catalyst, but substantial differences are apparent in the $\mathrm{P}(2 \mathrm{p})$ region. 'The spectrum for the $\mathrm{Ni}_{2} \mathrm{P} / \mathrm{Al}_{2} \mathrm{O}_{3}$ catalyst shows a peak at $134.7 \mathrm{eV}$ that is dramatically larger and at a higher binding energy than the phosphate peak for the $\mathrm{Ni}_{2} \mathrm{P} / \mathrm{SiO}_{2}$ catalyst $(134.3 \mathrm{eV})$. 'The large $\mathrm{P}(2 \mathrm{p})$ peak at $134.7 \mathrm{eV}$ for the $\mathrm{Ni}_{2} \mathrm{P} / \mathrm{Al}_{2} \mathrm{O}_{3}$ catalyst has a binding energy in the range for $\mathrm{P}$ in $\mathrm{AlPO}_{4}$ molecular sieves (134.6 to $134.8 \mathrm{eV}),{ }^{10}$ indicating that impregnated phosphorus is reacting with the $\gamma-\mathrm{Al}_{2} \mathrm{O}_{3}$ support to form a surface layer of $\mathrm{AlPO}_{4}$ during the 
synthesis of the supported $\mathrm{Ni}_{2} \mathrm{P}$. The hydrodesulfurization activity of a $\mathrm{Ni}_{2} \mathrm{P} /$ A1203 catalyst is just 37 percent of that of a $\mathrm{Ni}_{2} \mathrm{P} / \mathrm{SiO}_{2}$ catalyst under similar conditions. Future research will investigate in detail support effects for oxidesupported metal phosphide catalysts.

\section{Citations}

Layman KA, and ME Bussell. 2004a. "Infrared Spectroscopic Investigation of CO Adsorption on Silica-Supported Nickel Phosphide Catalysts." Journal of Physical Chemistry B 108(30):10930 - 10941.

Layman KA, and ME Bussell. 2004b. "Infrared Spectroscopic Investigation of Thiophene Adsorption on Silica-Supported Nickel Phosphide Catalysts." Journal of Physical Chemistry B 108(40):15791 - 15802.

Oyama ST. 2003. "Novel Catalysts for Advanced Hydroprocessing: Transition Metal Phosphides." Journalof Catalysis 216(1-2):343 - 352.

Phillips DC, SJ Sawhill, R Self, and ME Bussell. 2002. "Synthesis, Characterization, and Hydrodesulfurization Properties of Silica-Supported Molybdenum Phosphide Catalysts." Journal of Catalysis 207(2):266 - 273.

RodriguezJA, JY Kim,JC Hanson, SJ Sawhill, and ME Bussell. 2003. "Physical and Chemical Properties of $\mathrm{MoP}, \mathrm{Ni}_{2} \mathrm{P}$, and $\mathrm{MoNiP}$ Hyrdrodesulfurization Catalysts: Time-Resolved X-ray Diffraction, Density Functional, and Hydrodesulfurization Activity Studies." Journal of Physical Chemist y B 107(26):6276 - 6285.

Sawhill SJ, KA Layman, D Van Wyk, M H Engelhard, C Wang, and ME Bussell. 2005. Journal of Catalysis (in press).

Sawhill SJ, DC Phillips, and ME Bussell 2003. "Thiophene Hydrodesulfurization Over Supported Nickel Phosphide Catalysts." Journal of Catalysis 215(2):208 - 219.

\section{Field Deployable EMSL Capabilities for Chemical and Physical Environmental Measurements}

ML Alexander, ${ }^{(a)}$ T B Jobson, ${ }^{(a)}$ MJ Molina, ${ }^{(b)}$ MT Molina, ${ }^{(b)}$ KS Johnson, ${ }^{(b)}$ P Daum, ${ }^{(\mathrm{c})}$ H Westberg, ${ }^{(\mathrm{d})}$ B Lamb, ${ }^{(\mathrm{d})}$ C Spicer, ${ }^{(\mathrm{e})}$ P Palmer, ${ }^{\left({ }^{(}\right)}$and T Mark ${ }^{(\mathrm{g})}$

(a) Pacific Northwest National Laboratory, Richland, Washington

(b) Massachusetts Institute of Technology, Cambridge, Massachusetts

(c) Brookhaven National Laboratory, Upton, New York

(d) Washington State University, Pullman, Washington

(e) Battelle Memorial Institute, Columbus, Ohio

(f) San Francisco State University, San Francisco, California

(g) University of Innsbruck, Innsbruck, Austria 


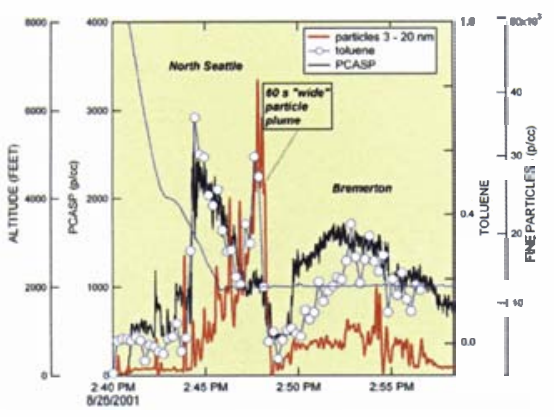

Figure 4-104. Proton transfer reaction mass spectrometer measurements aboard Battelle's GI research aircraft in Seattle.

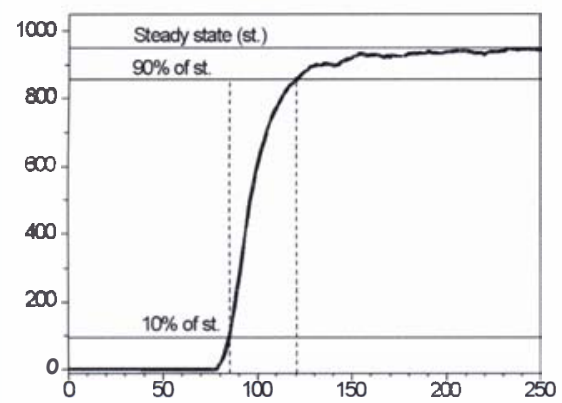

Figure 4-105. Proton transfer reaction mass spectrometry response to benzene through the membrane when switching from zero air to sample air.
Research areas for remote deployment of the proton transfer reaction mass spectrometer and aerosol mass spectrometer have included atmospheric chemistry (Jobson et al. 2004), diesel engine characterization, and emissions from the explosion of military ordnance and inhalation toxicology (Anderson et al. 2003). Laboratory studies using these capabilities at EMSL have included ion-molecule reactions, characterization of membrane materials (Alexander et al. 2003; Boscaini et al. 2004), and methods for improving the instruments themselves (Prazeller et al. 2003).

\section{Proton Transfer Reaction Mass Spectrometer}

'This instrument is capable of fast, real-time, online quantification of volatile organic compounds in air. Detection limits for a one-second integration time range from $50 \mathrm{pptv}$ to about $300 \mathrm{pptv}$, depending on the nature of the analyte. 'The response time of the instrument is about one second. Organic species are measured by chemical ionization mass spectrometry using $\mathrm{H}_{3} \mathrm{O}^{+}$as the reagent ion yielding ionization with negligible fragmentation. Organic species having a proton affinity greater than that of $\mathrm{H}_{2} \mathrm{O}$ will react with $\mathrm{H}_{3} \mathrm{O}^{+}$in a proton transfer reaction:

$$
\mathrm{R}+\mathrm{H}_{3} \mathrm{O}^{+--}>\mathrm{RH}^{+}+\mathrm{H}_{2} \mathrm{O}
$$

'The major constituents of air do not react with $\mathrm{H}_{3} \mathrm{O}^{+}$. A small portion of the flow through the reaction chamber is sampled by a quadrupole mass spectrometer, where the $\mathrm{RH}^{+}$ions are mass filtered and detected by an ion multiplier. "The amount of analyte $(\mathrm{R})$ in the sample air is determined by a simple formula relating the $\mathrm{H}_{3} \mathrm{O}^{+}$count rate, the $\mathrm{RH}^{+}$count rate, the rate constant for the ion-molecule reaction, and a fixed reaction time.

EMSL's proton transfer reaction mass spectrometer has been deployed for atmospheric studies both as a ground-based instrument and on Battelle's G-1 research aircraft. Measurements have been made in Seattle, Mexico City, and LaTrobe, Pennsylvania, as part of large air quality studies. Figure 4-104 shows a time series of toluene measurements made by the instrument as the Battelle G-1 aircraft flew west across Seattle towards the town of Bremerton, Washington. Measurements of toluene were made every 15 seconds with a dwell time of two seconds. The black trace shows the 0.1 - to $3-\mu \mathrm{m}$ particle concentration, while the red trace shows the 3- to 20-nm particle concentration. "This latter particle size range is indicative of newly formed particles. 'The spike in this size range at 2:48 p.m. coincides with a spike in toluene concentration, suggesting evidence for the role of anthropogenic hydrocarbons in new particle formation.

Laboratory-based studies using this instrument have included the characterization of semi-permeable membrane materials, including measurements of diffusion coefficientsand partition coefficients for various chemical species of interest. Figure 4-105 shows a typical proton transfer reaction mass spectrometry response to the introduction of a sample thorough a semi-permeable membrane. The rise time (from 10 and 90 percent of the steady state) and steady-state concentration inside the membrane can be derived from these data and used to calculate the diffusion coefficient and partition coefficient. 
Researchers are working to develop the next-generation proton transfer reaction mass spectrometer. The new instrument uses an ion-trap mass spectrometer in place of a linear quadrupole and boasts faster time response and the ability to resolve isobaric interferences. A laboratory prototype has been constructed, and external funding from DOE's Atmospheric Science Program has been granted to further develop the new proton transfer ion trap mass spectrometer and deploy it on Battelle's G1 research aircraft.

\section{Aerosol Mass Spectrometer}

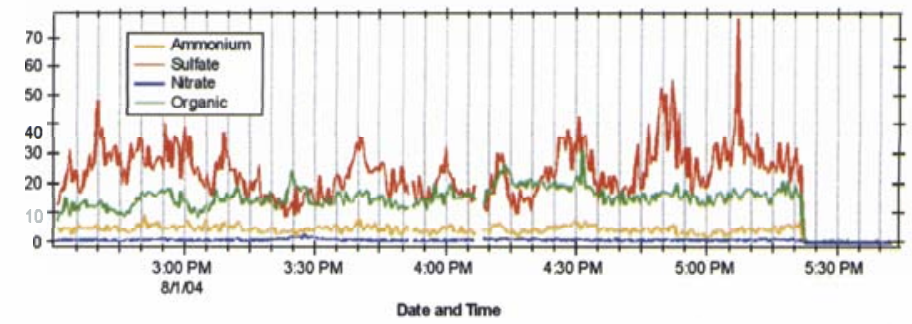

This state-of-the-art instrument simultaneously measures the size and chemical composition of aerosols in real time. Figure 4-106 shows data taken by the aerosol mass spectrometer aboard Battelle's G1 research aircraft for the 2004 New England Air Quality Study.

\section{Citations}

Alexander ML, E Boscaini, W Lindinger, and T D Mark. 2003. "Membrane Introduction Proton-Transfer-Reaction Mass Spectrometry." InternationalJournal of Mass Spectrometry 223-224:763-770.

Anderson GM, B Westerberg, and ML Alexander. 2003. 'Vapor Phase Analysis of Mainstream Tobacco Smoke by Proton Transfer Reaction Mass Spectrometry (PTRMS)." Presented by Greg Anderson at the Meeting of the Tobacco Science Research Conference, Norfolk, Virginia, on September 22,2003.

Boscaini E, ML Alexander, P Prazeller, and T D Mark. 2004. 'Investigation of Fundamental Physical Properties of a Polydimethylsiloxane (PDMS) Membrane using Proton Transfer Reaction-Mass Spectrometry (PTR-MS)." International Journal of Mass Spectrometry 239(2-3):179-186.

Jobson BT, ML Alexander, P Prazeller, CM Berkowitz, RM Volkamer, L Molina, H H Westberg, BK Lamb, RE Valasco, and EJ Allwine. 'Hydrocarbon Measurements during the MCMA2003 Field Experiment - Intercomparison and Analysis." Presented by Michael Alexander at the American Geophysical Union Conference, San Francisco, California, on December 14,2004.

Prazeller P, P T Palmer, E Boscaini, T Jobson, and ML Alexander. 2003. 'Proton Transfer Reaction Ion Trap Mass Spectrometer." Rapid Communications in Mass Spectrometry 17(14):1593-1599. 
oxidase-based amperometric biosensors. Glucose sensor, a model biosensor, was also developed based on the immobilization of glucose oxidase/Nafion/CNT on glassy carbon and gold electrodes as part of this research.

Nanosensors based on carbon nanotube nanoelectrode arrays have also been successfully fabricated from low site density-aligned carbon nanotubes (Figure 4-107). The carbon nanotubes were grown by plasma-enhanced chemical vapor deposition on nickel nanoparticles made by electrochemical deposition. Each nanotube is separated from the nearest neighbor by several microns. 'The nanoelectrode arrays, consisting of up to millions of individual nanoelectrodes with diameters of $50 \mathrm{~nm}$, were made in a $1-\mathrm{cm} 2$ area by this nonlithography method. Electrochemical characterization such as cyclic voltammetry and square wave voltammetry was performed to characterize these nanoelectrode arrays.

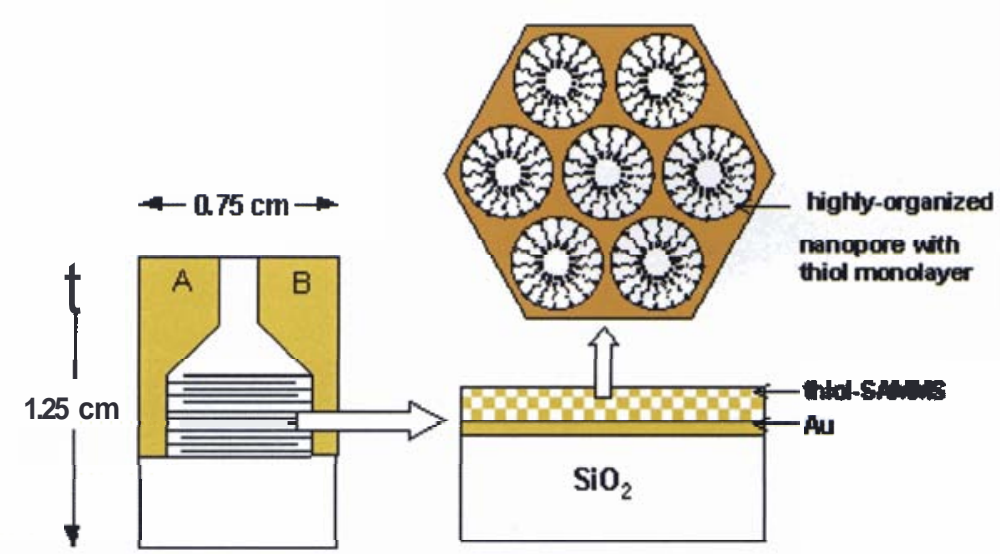

In addition, biosensors based on immobilization of enzymes, DNA, and antibodies have also been successfully developed for biosensing applications in biomedical, environmental, and homeland security applications.

In related research, various self-assembled monolayers on mesoporous silica (SAMMS) with selective functional ligands for toxic heavy metals and actinides have been developed using the capabilities of EMSL. Recently, we successfully coated the SAMMS thin-films on electrode surfaces to form selective nanoengineered electrochemical sensors for detection of copper, cadmium, lead, mercury, and uranium (Figure 4-108). The microchip gold electrode with SAMMS thin-film was integrated in a microfluidic system. Heavy metals or radionuclides were selectively preconcentrated on SAMMS thin-films when sample solution continuously flowed through the electrode surface. A clean electrolyte solution was used to remove the sample matrix from the electrode surface. Square-wave voltammetry was used to quantify the surface-bound metal species. 'The SAMMS-based electrochemical sensors were demonstrated to be quite selective for simultaneous detection of copper, cadmium, lead, and mercury with a detection limit at part per billion level. These results demonstrate that SAMMS-based electrochemical sensors are quite promising for analysis of toxic metals and radionuclides. 


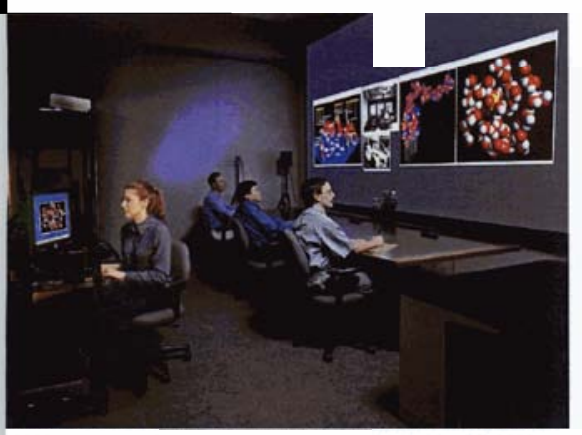

EMSL's Molecular Science Computing Facility is equipped with a Graphics and Visualization Laboratory that allows users to visualize and analyze large data sets generated by the facility's computing resources.
4.1.6

\section{Molecular Science Computing Facility}

'Ihe Molecular Science Computing Facility supports a wide range of computational activities in environmental molecular research, from benchmark calculations on small molecules to reliable calculations on large molecules, from solids to simulations of large biomolecules, and from reactive chemical transport modeling to regional cloud climate modeling. "The facility provides an integrated, high-performance production computing environment with links to external facilities and laboratories within the DOE system, collaborating universities, and industry.

\section{Representative Research Activities}

The Molecular Science Computing Facility provides massively parallel computational resources and expertise to researchers at hundreds of sites across the world to conduct research and develop applications, such as groundwater flow simulations; climate models; geochemical applications; modeling of chemistry on porous sites; heavy element chemistry; molecular thermodynamics and kinetics; prediction of excited states; nanotechnology; catalysis; biological membranes; and bioinformatics. Staff of the Molecular Science Computing Facility also develop extremely scalable software such as the award-winning Molecular Science Software Suite. A Graphics and Visualization Laboratory within this facility provides researchers with visualization capabilities of large data sets generated by the facility's computing equipment.

\section{Research Capabilities}

- Production cluster of 978 Hewlett Packard rx2600 nodes, 1956 1.5-gigahertz IA64 processors, 450 terabytes of local disk, 6.8 terabytes of memory, and 11.8 teraflops of theoretical peak performance

Shared cluster file system called /dtemp, 53 terabytes in size

- EMSL long-term data store, 300 terabytes in size

- Network comprised of OC12 (600 MBit/sec) internet connection, Gigabit Ethernet

- Visualization server comprised of Silicon Graphics Incorporated Onyx 3400 Graphics, eight processors, 16 gigabtyes of memory, two Infinite Reality3 pipes, 144 gigabytes of disk space, with a PanoramTech three-screen monitor

- Digital video editing suite

- Molecular Science Software Suite comprised of:

' Northwest Chemistry Software (NWChem), the massively parallel computational chemistry software that performs quantum, classical, and combined quantum/classical computations in molecular chemistry and structural biology 
${ }^{\circ}$ Extensible Computational Chemistry Environment (Ecce), a problemsolving environment for setting up, running, and analyzing chemistry computations

${ }^{\circ}$ Global Array (GA) Tools, an efficient and portable "shared-memory" programming interface for distributed-memory computers.

\section{Future Directions}

In 2005, the Molecular Science Computing Facility will be a stable production facility focused on accomplishinglarge, impactful science, including support for Computational Grand Challenges and Pilot Projects, as well as EMSL Scientific Grand Challenges and continuing support and development of the Molecular Science Software capabilities.

The planned lifecycle for the facility's primary computing resource is three to four years (the current supercomputerwill be four years old in June 2007). As of April 2005, work is nearing completion on a Greenbook that describes the evolving science drivers for performing environmental molecular research at EMSL and provides guidance associated with the next generation of highperformance computing that must be developed to address this critical research. Once completed, the MSCF Greenbook will guide procurement of the nextgeneration supercomputer and associated supporting equipment. The intent is to render the replacement computer operational in time to allow six months of overlap between the new computer and the old to facilitate user migration in a nondisruptive manner.

Software improvements will center on the science areas described in the EMSL vision and algorithms specificallyidentified in the Greenbook activity. In addition to porting, debugging, and improving the scalability of the software, improvements will be made in the cost of high-accuracy computations (especially with respect to excited states) and enabling simulations that more closely resemble complex experimental conditions. Problem-solving environment improvements will include additional interfacing with molecular dynamics and planewave computations. Software tutorials and manuals will continue to be updated to ease the use of the software.

In addition to hardware and software improvements described above, the Molecular Science Computing Facility will continue to provide expert staff who possess knowledge and experience in many areas of high-performancecomputing and are also specialists in the scientific areas of focus. The facility team will remain dedicated to providing a complete production environment that efficiently and effectively allows researchers to solve large scientific challenges.

\section{Facility Staff}

\section{Visualization, User Services, and Software}

'Theresa L. Windus

Technical Lead (509) 376-4529 theresa.windus@pnl.gov
Wibe A. de Jong

Senior Research Scientist

(509) $376-5290$

wibe.dejong@pnl.gov 
Jessica M. Foreman

Administrator

(509) 376-3412

jessica.foreman@pnl.gov

Bettina M. Foley

Administrator

(509) 376-2767

tina.foley@pnl.gov

Yuri Alexeev

Postdoctoral Research Fellow

(509) 376-5152

yuri.alexeev@pnl.gov

Edoardo Aprà

Senior Research Scientist

(509) 376-1280

edoardo.apra@pnl.gov

Doug J. Baxter

Senior Research Scientist

(509) 376-3751

douglas.baxter@pnl.gov

\section{Operations}

Kevin M. Regimbal

Technical Lead

(509) 376-2246

kevin.regimbal@pnl.gov

David E. Cowley

Senior Research Scientist

(509) 376-9181

david.cowley@pnl.gov

Evan J. Felix

Senior Research Scientist

(509) 376-1491

evan.felix@pnl.gov

Kevin M. Fox

Research Scientist

(509) 376-4465

kevin.fox@pnl.gov

Brandon H. Hayden

LTE College Student

(509) 376-1493

brandon.hayden@pnl.gov
Mahin Hackler

Scientist

(509) 376-2746

mahin.hackler@pnl.gov

Karol Kowalski

Senior Research Scientist (509) 376-6751

karol.kowalski@pnl.gov

Jun Li

Senior Research Scientist (509) 376-4354

jun.li@pnl.gov

Marat Valiev

Senior Research Scientist (509) 376-2514

marat.valiev@pnl.gov

Erich R. Vorpagel

Chief Scientist

(509) 376-0751

erich.vorpagel@pnl.gov

Cindy Marasco

Senior Research Scientist (509) 376-1241

cindy.marasco@pnl.gov

Ryan W. Mooney

Senior Research Scientist (509) 376-3590

ryan.mooney@pnl.gov

Kenneth P. Schmidt

Technician

(509) 376-4178

kenneth.schmidt@pnl.gov

Gary B. Skouson

Senior Research Scientist

(509) 376-5401

gary.skouson@pnl.gov

Ryan P. Wright

Research Scientist

(509) 376-3052

ryan.wright@pnl.gov 
Scott M. Jackson

Senior Research Scientist

(509) 376-2205

scott.jackson@pnl.gov

\section{Key Staff}

Key staff, besides those listed above, include Gary D. Black, Eric J. Bylaska, Brett T. Didier, Todd O. Elsethagen, Jarek Nieplocha, Manojkumar Krishnan, Carina S. Lansing, Michael C. Perkins, Kenneth A. Perrine, Michael R.

Peterson, Karen L. Schuchardt, and T. P. Straatsma.

\section{Biomolecular Simulation of Base Excision Repair and Protein Signaling}

TP Straatsma, ${ }^{(a)}$ ER Vorpagel, (a) M Zacharias, ${ }^{(b)}$ PE Smith, ${ }^{(c)}$ CF Wong, ${ }^{(d)}$ JA McCammon, (d) JH Miller, ${ }^{(e)}$ and $\mathrm{K} \mathrm{Wei}{ }^{(\mathrm{e})}$

(a) Pacific Northwest National Laboratory, Richland, Washington

(b) Institute for Molecular Biotechnology, Jena, Germany

(c) Kansas State University, Manhattan, Kansas

(d) University of California, San Diego, California

(e) Washington State University, Pullman, Washington

Environmental factors, including ionizing radiation, contribute to continuous damage of cellular DNA, in addition to endogenous sources. The damage resulting from oxidative stress and ionizing radiation is primarily in the form of oxidized bases, single strand breaks, and loss of bases. 'These are the targets of the base excision repair mechanism enzymes, including polymerase 13. Failure to repair these damaged DNA sites correctly and in a timely manner can result in cell death, carcinogenesis, or genetic diseases. Resulting mutations in cell signal transduction enzymes can lead to uncontrolled cell proliferation or differentiation. For example, mutations in Ras, the molecular switch in several growth-factor signaling pathways, have been found in about 30 percent of human tumors. 'These signaling pathways often involve a chain of protein kinases that activate or deactivate each other through phosphorylation reactions, eventually controlling the activation of transcription factors in the cell nucleus. 'This research seeks the massively parallel computing resources required to perform molecular modeling and simulation studies to enhance our understanding of the mechanism of human polymerase 13, one of the key enzymes in base excision repair, and the cell signaling enzymes cyclic-adenosine monophosphate (AMP)dependent protein kinase and Ras. Specifically, this work will focus on the:

- dynamics of DNA and damaged DNA

- dynamics and energetics of base flipping in DNA 


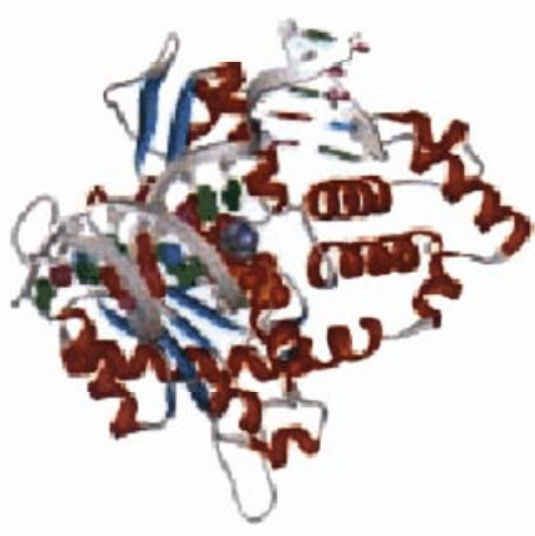

Figure 4-109. Polymerase active site of the DNA repair enzyme, polymerase $B$.
- co-solvent effects on biomolecular structures, including DNA mechanism and fidelity of nucleotide insertion by base excision repair enzyme human polymerase 13

- mechanism and inhibitor design for cyclic-AMP-dependent protein kinase

- dynamics and energetics of Ras and its complex with effector molecule Raf for the molecular dynamics simulations and electronic structure calculations.

The proposed investigations will rely on NWChem, the massively parallel software for computational chemistry developed at EMSL. NWChem will also be used for the analysis of the generated molecular dynamics trajectories, and any additional analysis tools required for the proposed work will be developed within the analysis modules of NWChem.

\section{Characterization of the Active Site of DNA Polymerase ß}

It is well established that the fully formed polymerase active site of the DNA repair enzyme, polymerase 13 , including two bound $\mathrm{Mg}^{2}+$ cations and the nucleoside triphosphate substrate, exists at only one point in the catalytic cycle just prior to the chemical nucleotidyl transfer step. The structure of the active conformation has been the subject of much interest as it relates to the mechanism of the chemical step and also to the question of fidelity assurance. While crystal structures of ternary polymerase $B$ - (primer-template)DNA- deoxyribonucleoside triphosphate (dNTP) complexes have provided the main structural features of the active site, they are necessarily incomplete due to intentional alterations (e.g., removal of the $3^{\prime} \mathrm{OH}$ groups from primer and substrate) that were needed to obtain a structure from mid-cycle. Working from the crystal structure closest to the fully formed active site (pdb: 1bpy), molecular dynamics simulations of the solvated ternary complex were performed, with the missing 3'OHs restored, via modeling, to the primer and substrate, and without restoration of the $3^{\prime} \mathrm{OHs}$. The results of these simulations, taken together with ab initio optimizations on simplified active site models, indicate that the missing primer $\mathbf{3}^{\prime} \mathbf{O H}$ in the crystal structure is responsible for a significant perturbation in the coordination sphere of the catalytic cation and allow us to suggest several corrections and additions to the active site structure as observed by crystallography. In addition, the calculations help to resolve questions that have been raised regarding the protonation states of coordinating ligands (Figure 4-109). 


\title{
Biomolecular Interfaces: Simulation of Sedimentation of Biomolecular Assemblies
}

\author{
AD Haymet, (a) BW Beck, (a) GC Lynch, ${ }^{(a)}$ BM Pettitt, (a) TC Rogala, (a) and \\ K Wong ${ }^{(a)}$
}

(a) University of Houston, Houston, Texas

The goal of this project is to provide a comprehensive computational model, spanning many length and time scales, of the characteristics of biological molecular assemblies, such as associating proteins, lipid vesicles, and viruses, when placed in a modern analytical ultra-centrifuge. Sedimentation equilibria and velocities for polydisperse and self-assembling systems will be studied. This problem has an explicit coupling of length scales from the microscopic correlations between molecules in the assembly to the shape of the resulting assembly to the hydrodynamic flow around the irregularly shaped bodies during sedimentation. The results will be tested against experiments and checked for consistency with known thermodynamic and structural data.

\section{Computationally Useful Bridge Diagram Series}

In many-body theory, bridge diagrams are a set of topologically dense, large dimensional integrals of interest in the theoretical study of liquids. We calculated a new series for simple fluid mixtures of argon and krypton. Our results were both numerically encouraging for this system, and theoretically encouraging for the ongoing extension to more complex fluids and solvation studies (Figure 4-110) (Dyer et al. 2002; Perkyns et al. 2002).

\section{Base-pairing on Surfaces for Untethered Oligonucleotides}

Recent designs of DNA microarrays use nontethered DNA probes, which are bound to a positive charged surface by nonbonded, electrostatic interactions. They show specific, high-affinity binding of large targets with relatively rapid kinetics. A 10-ns all-atom molecular dynamics simulation of the system was performed and found that the probe was bound to the surface primarily with the phosphates attracted to the surface. The bases remained stacked and pointed preferentially upward, available for hybridization with complementary target strand (Figure 4-111) (Belosludtsev et al. 2001).

\section{Solvation of Biomolecular Interfaces: Role of Solvent in S. marcescens Endonuclease Dimerization}

Simulations have revealed the presence of a solvent-filled channel leading from the active site cavity of the enzyme to three distinct openings near the dimer interface. The presence of this channel has implications to both protein stability and regulation of enzymatic activity. This channel appears to have an intermittent barrier midway down its length near residues Ile174, Ala186, and

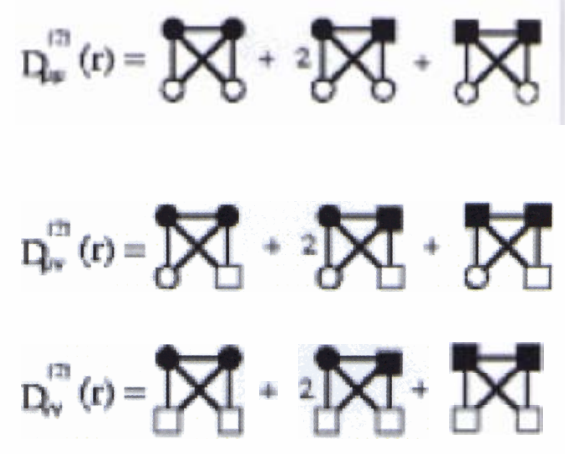

Figure 4-110. Theoretical bridge diagrams for a set of topologically dense, large dimensional integrals of Interest in study of liquids.

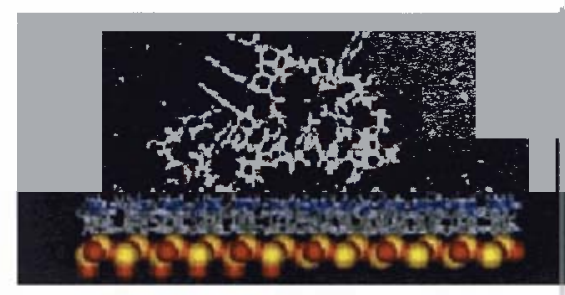

Figure 4-111. Molecular dynamics simulation of basepairing on surfaces for untethered oligonucleotides. 


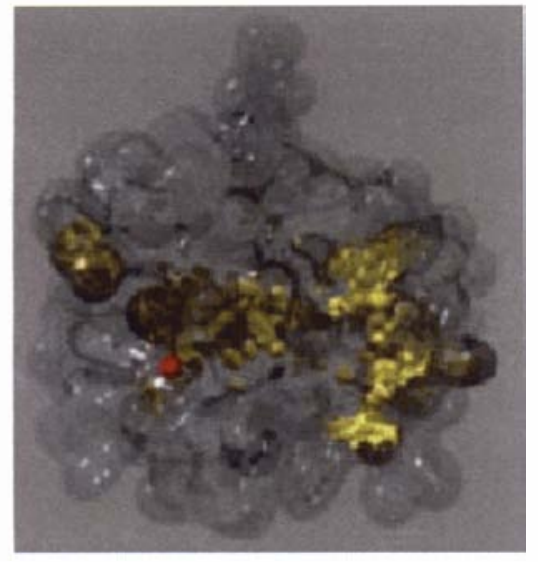

Figure 4-112. Average channel solvent density in enzyme monomer over 1 ns.
Phe188 that acts as a "gate ${ }^{n}$ to control the diffusion of water between the active and the dimer interface. The behavior of this gate appears to be important in the function of this enzyme and may help explain the evolution of the dimeric state for this system (Figure 4-112).

Citations

Belosludtsev Y, B Iverseon, S Lemeshko, R Eggers, R Wiese, S Lee, T Powdrill, and M Hogan. 2001. "DNA Microarrays Based on Noncovalent Oligonucleartide Attachment and Hybridization in Two Dimensions." Analytical Biochemistry 292(2):250256.

Dyer K, J Perkyns, and BM Pettitt. 2002. "Computationally Useful Bridge Diagram Series. III. Lennard-Jones Mixtures." Journal of Chemical Physics 116(21):9413-9421.

Perkyns JS, KM Dyer, and BM Pettitt. 2002. "ComputationallyUseful Bridge Diagram Series. II. Diagrams in h-bonds." Journal of Chemical Physics 116(21):9404-9412.

\section{Chemical Fate of Contaminants in the Environment: Chlorinated Hydrocarbons in the Groundwater}

DG Truhlar, (a) CJ Cramer, ${ }^{(a)}$ JD Thompson, ${ }^{(a)}$ AW Jasper, ${ }^{\left({ }^{(a)}\right.}$ BJ Lynch, ${ }^{(a)}$ NE Schultz, ${ }^{\left({ }^{(a)}\right.} \mathrm{C}$ Zhu, ${ }^{(\text {a) }}$ A Chakraborty, ${ }^{(a)}$ YA Borisov, ${ }^{(b)}$ K Morokuma, ${ }^{(c)}$ IV Khavrutskii, ${ }^{(c)}$ SJ Mo, ${ }^{(c)}$ S Irle, ${ }^{(c)}$ QWang, ${ }^{(c)}$ G Zheng, ${ }^{(c)}$ HA Witek, ${ }^{(c)}$ J Liu, ${ }^{(c)}$ A Dutta, ${ }^{(c)}$ P Zhang, ${ }^{(c)}$ D Quinonero, ${ }^{(c)}$ K Omiya, ${ }^{(c)}$ DG Musaev, ${ }^{(c)}$ TP Straatsma, ${ }^{(d)}$ M Dupuis, ${ }^{(d)}$ BC Garrett, ${ }^{(d)}$ and DT Chang ${ }^{(d)}$

(a) University of Minnesota, Minneapolis, Minnesota

(b) Russian Academy of Science, Moscow, Russia

(c) Emory University, Atlanta, Georgia

(d) Pacific Northwest National Laboratory, Richland, Washington

Chlorinated hydrocarbons, which can include carcinogens, are the most common contaminants found at hazardous waste sites, and are also the most prevalent contaminants at DOE sites that were involved in production of nuclear weapons. They are resistant to biodegradation, but can degrade by abiotic processes such as hydrolysis, nucleophilic substitution, and dehydrochlorination. Unfortunately, disagreement still exists about the mechanisms and rates of many of the key reactions. Our work is focused on modeling the reactions involved in the degradation of chlorinated hydrocarbons, in the groundwater. 'There are three primary goals for this research: 1) development of a computational approach that will allow reaction pathways and rate constants to be accurately calculated;2) development of more approximate approaches, evaluated against the more accurate approach, which will lay the groundwork for exploratory studies of more complex 
chlorinated hydrocarbons; and 3) application of these approaches to study the degradation pathways of chlorinated hydrocarbons in aqueous liquids. A major focus of this work is on the development of continuum solvation models, which are based on the solvation models of Cramer and Truhlar (1995) and are appropriate for calculations using DFT. Applications are focused on the initial elementary steps in reactions of hydroxide with chlorinated methane molecules, $\mathrm{CH}_{\mathrm{n}} \mathrm{Cl}^{(4-n)}$, and studies of reactions of hydroxide with chlorinate ethylene molecules, $\mathrm{C}_{2} \mathrm{H}_{\mathrm{n}} \mathrm{Cl}^{(4-\mathrm{n})}$.

Significant progress has been made toward developing a continuum solvation methodology needed to simulate the reactions of hydroxide with chlorinated hydrocarbons. In our initial work, the need to use diffuse functions to obtain accurate thermochemical simulations became clear. However, our solvation and charge models were not stable when diffuse functions were used. To solve this problem, we first derived a new type of population analysis that was stable to diffuse functions (Thompson et al. 2002), then we used this new type of population analysis to develop charge models that provide stable charges with diffuse basis sets (Winget et al. 2002; Thompson et al. 2003). We are making excellent progress in using the new charge models to create new solvation models. "This methodological development will be used on the originally plannea large computations. Although it was more efficient to perform preliminary work on desktop workstations and computer servers, the Computational Grand Challenge work on this project using this methodology requires the supercomputer located at EMSL.

Use of the supercomputer has been essential for the applications to the reactions of $\mathrm{OH}$ - with chlorinated methane molecules, $\mathrm{CH}_{n} \mathrm{Cl}_{(4-\mathrm{n})}$ with $\mathrm{n}=1-4$. In previous work, we completed a hierarchy of calculations for the gas-phase reactions for all values of $\mathrm{n}$ and for microsolvated reactions (with up to two water molecules) for $n=1-3$. Recent work on this project has focused primarily on the high-level electronic structure calculations (e.g., methods that include electron correlation such as second-order Møller-Plesset perturbation theory and couple cluster methods) for the microsolvated reactions of carbon tetrachloride $(n=4)$, which are a significant computational challenge and require the use of advanced software such as NWChem on the massively parallel computers. 'These calculations show a significant increase in the reaction barrier for the nucleophilic substitution reaction as the number of water molecules is increased.

To account for the effects of bulk solvation (i.e., increase of the amount of water molecules to a macroscopic number), we use a computationally tractable and efficient procedure for the calculation of potentials of mean force using mixed Hamiltonian models of electronic structure where quantum subsystems are described with computationally intensive ab initio wave functions. The mixed Hamiltonian is mapped into an all-classical Hamiltonian that is amenable to a thermodynamic perturbation treatment for the calculation of free energies. A small number of statistically uncorrelated (solute-solvent) configurations are selected from the Monte Carlo random walk generated with the all-classical Hamiltonian approximation. 'Those configurations are used in the averaging of the free energy using the mixed quantum/ classical Hamiltonian. In Figure 4-113, the dashed and solid lines with circles are 


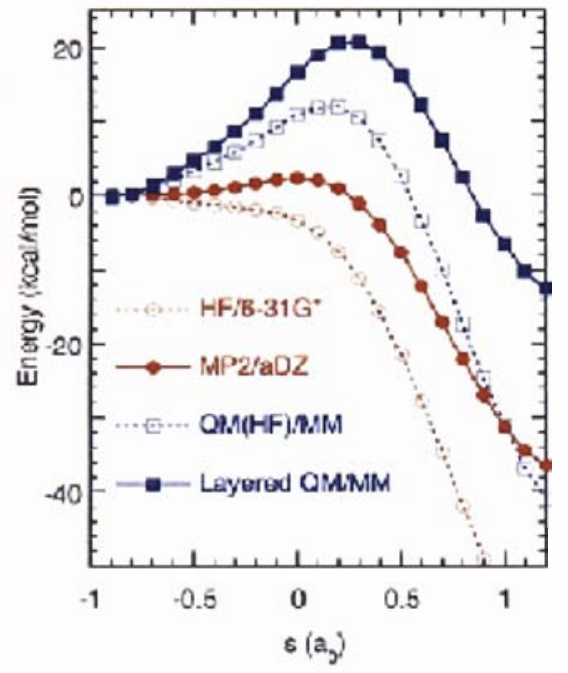

Figure 4-113. Application of this methodology is illustrated for the nucleophilic substitution reaction of methyl chloride by hydroxide. calculations of the energies for the gas-phase reaction using Hartree-Fock theory with a $6-31 \mathrm{G}^{*}$ basis set (dashed curve with open circles) and MøllerPlesset perturbation with an aug-cc-pVDZ basis set (solid curve with filled circles). 'The energies are computed at geometries along a minimum energy path for the gas-phase reaction that is calculated using Møller-Plesset perturbation/aDZ theory. 'The dashed line with open squares shows the results of bulk solvation on the Hartree-Fock $/ 6-31 \mathrm{G}^{*}$ results using the quantum mechanical/molecular mechanical approach described above. 'The most accurate value of the solvated potential energy curve is given by combining the Møller-Plesset perturbation results with those of the quantum mechanical (Hartree-Fock)/molecular mechanical and Hartree-Fock/6-31G ${ }^{*}$ results, using a layered electronic structure approach, to give the solid curve with filled squares.

Another aspect of this work is the development of efficient DFT methods to study chemical reactivity. In another research effort at the University of Minnesota, we are developing new force fields for aluminum nanoparticles that are important to energy research because of their potential as high-energy materials. The computer hardware and software at EMSL has been essential to the latest stage of this work.

In particular, we have been studying the transition from small-molecule properties to their bulk limit in aluminum molecules, clusters, and nanoparticles, specifically the evolution of the binding energy per particle as the cluster gets bigger. We also studied the multiplicities of the clusters. We recently developed a new effective core potential method for use with hybrid DFT that provides accurate energies and geometries. 'This development was performed using computers at the University of Minnesota, but we have been able to use the resulting technology to run large production runs on $\mathrm{Al}_{19}, \mathrm{Al}_{43}, \mathrm{Alss}, \mathrm{Al}_{79}$, and $\mathrm{Al}_{87}$ on EMSL's supercomputer using NWChem. For nanoparticle-sized aluminum (more than 50 atoms), the capabilities of the software and hardware are impressive. Therefore, we have been taking advantage of the parallel capabilities of NWChem and anticipate running even larger systems that will take full advantage of the computing power of EMSL for this kind of research.

\section{References}

Cramer CJ, and DG Truhlar. 1995. "Continuum Solvation Bottles: Classical and Quantum Mechanical Supplementations."In Reviews in Computational Chemistry, eds. KB Lipkowitz and DB Boyd, vol. 6, pp. 1-72. John Wiley and Sons, New York.

Thompson JD, JD Xidos, T M Sonbuchner, CJ Cramer, and DG Truhlar. 2002. "More Reliable Partial Atomic Charges When Using Diffuse Basis Functions." Physical Chemistry Communications 5:117-134.

Thompson JD, CJ Cramer, and DG Truhlar. 2003. "Parameterization of Charge Model 3 for AM1, PM3, BLYP, and B3LYP." Journalof ComputationalChemistry 24(11):1291-1304.

Winget P, JD Thompson, JD Xidos, CJ Cramer, and DG Truhlar. 2002. "Charge Model 3: A Class IV Charge Model Based on Hybrid Density Functional Theory with Variable Exchange." Journal of Physical ChemistryA 106(44):10707-10717. 


\section{The Growth Morphology of the $\{100\}$ Surface of KDP (Archerite) on the Molecular Scale}

\author{
AG Stack, (a) JR Rustad, (a) JJ DeYoreo, ${ }^{(b)}$ TA Land, ${ }^{(b)}$ and WH Casey ${ }^{(a)}$ \\ (a) University of California, Davis, California \\ (b)Lawrence Livermore National Laboratory, Livermore, California
}

First-principle calculations were conducted to examine the surface morphology of the (100) face growth hillock of potassium dihydrogen phosphate $\left(\left[\mathrm{KNH}_{4}\right] \mathrm{H}_{2} \mathrm{PO}_{4}\right)$ (Figure 4-114), also known as KDP or archerite (Stack et al. 2004). KDP is a crystal structure used extensively in industrial applications (e.g., the National Ignition Facility at Lawrence Livermore National Laboratory) (Stack et al. 2004). To perform the calculations, the plane-wave pseudopotential DFT and Car-Parrinello $a b$ initio molecular dynamics functions of the NWChem software package were used. The plane-wave pseudopotential DFT function provides a good economy of calculation time, especially for nontransition metal systems as large as this one, yet provides accurate energy predictions. Step energies also were calculated using the difference between a step and a slab, with the same number of atoms and cell dimensions. The (100) surface energy was calculated by subtracting the total plane-wave pseudopotential energy of a bulk phase from the energy of the same cell, but with space added in the [100] direction, so that the area of the calculation and the number of atoms were the same. Detachment energies of $\mathrm{KH}_{2} \mathrm{PO}_{4}$ growth units adsorbed to steps were calculated by subtracting the energy of the step with the growth unit adsorbed from the energy of a step and the energy of the isolated growth unit. Typical simulations were run for approximately 2000 iterations at 5 atomic units per time step (1.2 x 10-16 seconds per time step) with steady-state reached after approximately 1000 iterations.

Calculated detachment energies of the (010)-facing step were +4.2 and +4.5 $\mathrm{eV}$ (for two different terminations of the step) and $+3.8 \mathrm{eV}$ for the $\{001\}-$ facing step. A more unfavorable detachment energy indicates a less-labile growth unit and a larger net rate of step advancement. Because the calculated detachment energies of the (010)-facing steps are larger than the (001)-facing step, this indicates that the (010)-facing step should have a higher net rate of growth. This finding is in qualitative agreement with experimental results. The detachment energies of subsequent growth units show that the energy stays approximately the same for the $(010)$ face, but goes up $(+5.1 \mathrm{eV})$ for the (001) face. Therefore, it is likely that in the slow (001) direction, the initial adsorption of new material to the step is limiting the rate, and once it has been adsorbed, the second unit adsorbs very quickly and reproduces the step. $\{010\}$-facing step, A termination

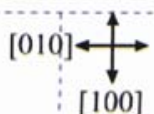

[100]
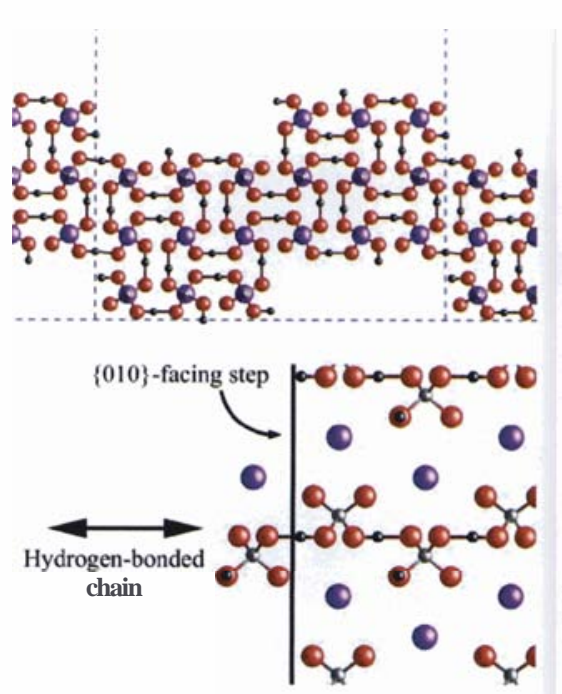

Figure 4-114. Atomic structures of the KDP surface. Potassium atoms are shown as purple spheres, oxygen atoms are red spheres, phosphorous atoms are grey spheres, and hydrogen atoms are black spheres. Hydrogen bonds are shown with alternating grey and white stripes linking the $\mathrm{H}_{2} \mathrm{PO}_{4}$ - molecules. Blue dashed lines indicate the supercell boundaries. Top: A step facing the (010) on the (100) surface, viewed down the [001] axis. Bottom: Same step viewed from looking down the [100] axis, with an adsorbed growth unit. Only the top-most layer is shown. The double-ended arrow shows the direction of hydrogen-bonded phosphate chains. 
published as a full paper in Nature Materials (Greeley and Mavrikakis 2004) and have been highlighted by the American Chemical Society news magazine Chemical E' Engineering News.

\section{Citation}

Greeley J, and M Mavrikakis. 2004. "Alloy Catalysts Designed from First Principles." Nature Materials 3(11):810-815. 


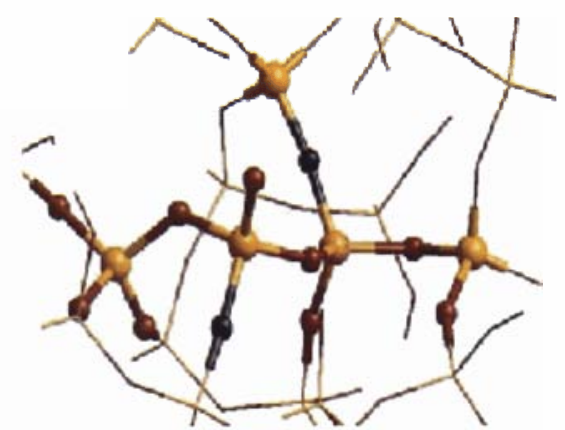

Figure 116a. Exciton-induced damage process in amorphous silica. Part of the 72 atom glass configuration. No coordination defects are present.

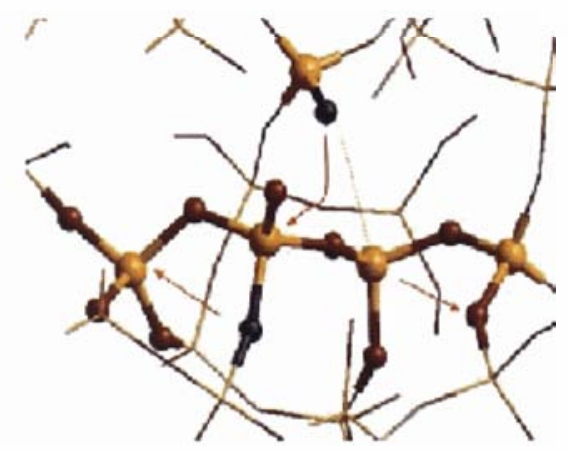

Figure 116b. Exciton-induced damage process in amorphous silica. Self-trapped triplet state exciton configuration. A siliconoxygen bond has been broken.

\section{Immobilization of Radionuclides in Oxides}

H Jonsson, ${ }^{(a)} \mathrm{F} \mathrm{Vila,}$, (a) K Tsemekhman, ${ }^{(a)}$ A Arnaldsson, ${ }^{(a)}$ L Arnadottir, ${ }^{(a)}$ MA

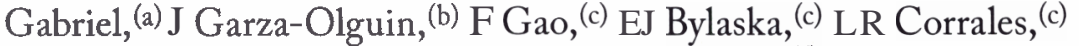
S Hirata, ${ }^{(d)}$ RM Van Ginhoven, ${ }^{(e)}$ and D Makarov ${ }^{(f)}$

(a) University of Washington, Seattle, Washington

(b) Universidad Autonoma Metropolitana, Iztapalapa, Irakium, Mexico

(c) Pacific Northwest National Laboratory, Richland, Washington

(d) W.R. Wiley Environmental Molecular Sciences Laboratory, Richland, Washington

(e) Commissariat a l'Energie Atomique, Gif Sur Yvette, France

(f) University of Texas, Austin, Texas

In this project, we are using computer simulations to study structural changes in oxide glasses that result from electronic excitations occurring over long time periods-for example, hydrolysis driven by radioactive decay.

At the most basic level, our project involves testing a methodology to establish what level of theory is needed to accurately reproduce electronic defects and chemical reactions in oxides, especially quartz and amorphous silica. We recently reported an extensive study of excitons in silica clusters calculated by both wave function methods and DFT (Van Ginhoven et al. 2003). It is clear from this and other studies that we performed that DFT calculations with functionals commonly used in condensed-phase simulations are inadequate for the study of excitons and electron holes. We found that the B3LYP functional is much superior, but it is too computationally demanding when extended systems are studied because of the need to evaluate exact exchange. Instead, we developed an algorithm and implementation in the NWChem software package for self-interaction correction - or SIC-to DFT. This is the first implementation that we know of that includes self-consistency and incorporates periodic boundary conditions to allow simulations of extended systems. Furthermore, our implementation includes atomic forces and, therefore, enables us to calculate minimum energy structures and transition states. The computational cost is, in principle, only a factor of 3 to 5 over regular DFT calculations, but more optimization of the code is needed before that theoretical limit can be achieved.

'The DFT-SIC calculations provide solutions to many of the problems associated with regular condensed-phaseDFT calculations, and our code opens the possibility of addressing various questions that could not be computationally addressed in the past. Several test problems indicate that, with some simple modifications, the DFT-SIC approach can give comparable accuracy for both stable structures and transition states as the much more expensive B3LYP functional. We applied the DFT-SIC method to several problems in oxides. One is the spin density of a hole created by substituting an aluminum-atom for a silicon-atom in quartz. Regular DFT, using local-density-approximation or generalized-gradient approximation functionals, shows a spin density that 
is delocalized over all four oxygen-atom neighbors of the aluminum-atom. However, experimental results show that the spin density is localized on one of the oxygen-atoms, as manifested, for example, by lengthening of only one of the aluminum-oxygen bonds. The DFT-SIC calculations indeed show localization on only one of the oxygen-atoms. Another example comes from triplet state excitons in quartz. With DFT/generalized-gradient approximation (GGA) calculations, the spin density of the lowest energy structure is distributed over all 72 atoms in the simulation cell. Localized excitons can, however, be found and have been studied in this project previously, but they are metastable with respect to the delocalized state.

With DFT-SIC, the localized excitons become lower in energy, which agrees with experimental findings. Finally, a similar reversal of the relative energy of localized and delocalized states is found for electron holes in amorphous silica. The DFT-SIC calculations show stable localized holes that agree with experimental results, while the holes delocalize at the DFT/GGA level of theory.

The relative energy and structure of different localized excitonic states in silica are still quite well described by DFT/GGA. We used this level of theory to study the formation of defects in silica due to exciton formation. Ten different glass samples that are free of coordination defects were generated (Figure 116a). The figure shows an unexpected damage process that was observed in one of the glasses. 'The damage was unexpected because no thermally activated process was required to form the defect. First, the system was excited to the triplet state, representing a fast process of initial excitation to some very high-energy singlet state and then intersystem crossing and trapping in the lowest triplet state. Because this is a long-lived state, the system was then relaxed in the triplet state, and a break in a silicon-oxygen bond was observed (Figure 116b). When the system was returned to the singlet state to represent radiative decay and then relaxed, the defect did not disappear, but rather developed into two coordination defects, 1) a fivefold coordinated silicon-atom and 2) a small, strained, fourmember ring consisting of two silicon-atoms and two oxygen-atoms (Figure 116c). The energy along this path was evaluated both in the singlet and triplet state, as shown in Figure 117.

We then searched for various transition paths from this state. A transition to the original defect-free structure involves a significant energy barrier of $0.76 \mathrm{eV}$. However, several other processes with similar activation energies could represent further migration of the defects. "This could, therefore, be the first step in the formation of an interstitial oxygen-atom defect. It is well known experimentally that radiation of glasses results in the formation of oxygen bubbles. Further simulation studies will address this issue and other defect formation mechanisms in glasses.

We have also studied the insertion of water molecules into glass samples that were free of coordination defects and found, surprisingly, that sites exist where the energy of the water molecule is comparable to the energy in liquid water. 'The barrier for dissociation of the molecule was found to be very small, on the order of $0.1 \mathrm{eV}$, and leads to a lower energy state with two hydroxyl groups. This represents an initial stage in hydrolysis of the glass. The importance

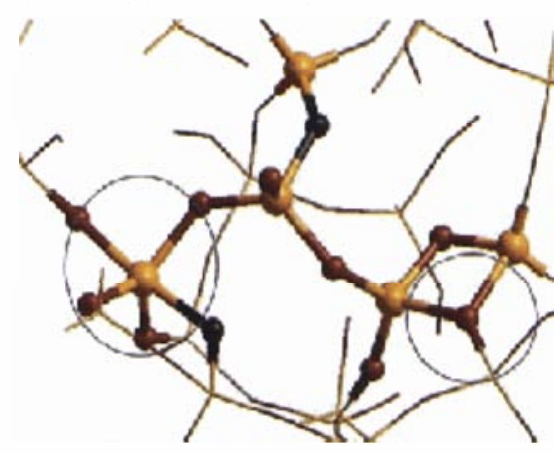

Figure 116c. Exciton-induced damage process in amorphous silica. Relaxed singlet, groundstate configuration after decay of the exciton. A fivefold siliconatom (circled left) and a small, strained, four-membered ring (circled right) are now present.

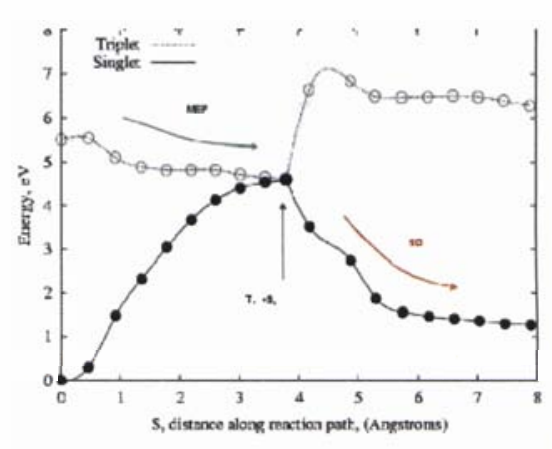

Figure 4-117. Excitoninduced damage process in amorphous silica. Energy of the singlet and triplet states for the configurational changes described in Figure 4-116 a-c: first a minimum energy path on the triplet state surface, and then a steepest descent path on the singlet state surface. 


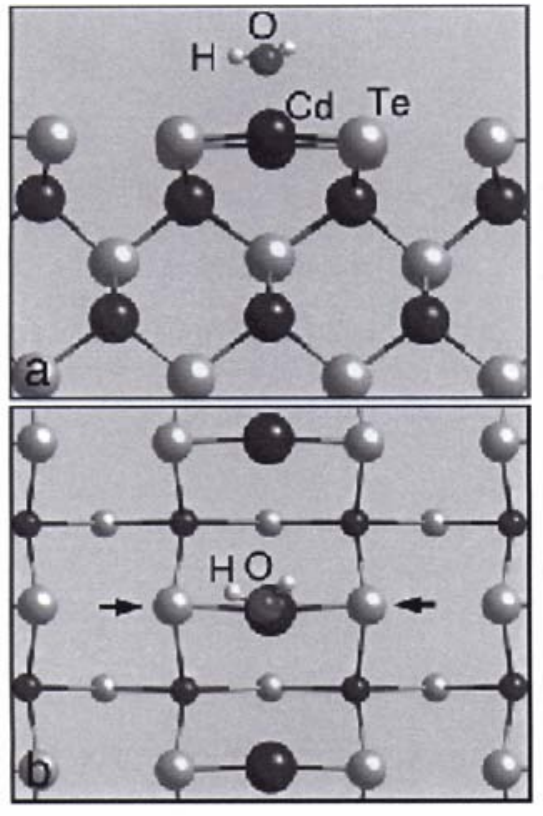

Figure 4-118. Side a) and top b) views of the structure of a water $\mathrm{m}$ olecule adsorbed on the perfect $(2 \times 1)$ reconstructed CdTe(001) surface. of strain and anomalous silicon-oxygen rings has been studied to identify particularly reactive sites and sites where electronic defects such as excitons and holes are likely to self-trap.

\section{Citation}

Van Ginhoven RM, H Jonsson, KA Peterson, M Dupuis, and LR Corrales. 2003. "An Ab Initio Study of Self-Trapped Excitons in Alpha-Quartz." Journal of Chemical Physics 118(14):6582-6593.

\section{Degradation Mechanism of Solar Cells in an Adverse Environment}

\author{
Y Yan, ${ }^{(a)}$ M Romero, ${ }^{(a)} \mathrm{C}$ Jiang, ${ }^{(a)}$ MM Al-Jassim, ${ }^{(a)}$ SJ Pennycook, ${ }^{(b)}$ and \\ G Duscher ${ }^{(c)}$ \\ (a) National Renewable Energy Laboratory, Golden, Colorado \\ (b) Oak Ridge National Laboratory, Oak Ridge, Tennessee \\ (c) North Carolina State University, Raleigh, North Carolina
}

CdTe is a promising candidate for large-scale, thin-film solar cells because or its nearly ideal bandgap, high-absorption coefficient, and ease of fabrication as a film. 'The structure of a typical $\mathrm{CdTe} / \mathrm{CdS}$ solar cell consists of a glass superstrate, then a thin layer of $\mathrm{SnO}_{2}$ (front contact), n-type CdS, p-type CdTe, and a back contact. Currently, low efficiency and degradation are the two major obstacles preventing further commercialization of this technology. It has been reported that the current-voltage characteristics of $\mathrm{CdTe} / \mathrm{CdS}$ solar cells degrade when the cells are exposed to moisture and oxygen over extended periods of time. Currently, two scenarios have been proposed to explain the degradation mechanism. 'The first scenario is that water vapor may cause peeling of the front contact and oxidization of the back contact. The second is that water molecules interact with CdTe and then dissociate and produce hydrogen or oxygen impurities, which diffuse into CdTe and alter its properties.

We investigated the adsorption of water molecules on the $\mathrm{CdTe}(001)$ surface by density-functional, total-energy calculations within the generalized gradient approximation. We found that dissociative adsorption of water molecules on $\mathrm{Cd} \mathrm{Te}(001)$ is energetically unfavorable-water can only adsorb molecularly on cadmium atoms. We also found that the formation of cadmium and tellurium vacancies on the surface and electron and hole doping do not change the adsorption behavior of water on the $\mathrm{CdTe}(001)$ surface. Our results suggest that the first scenario could be the mechanism for moisture-induced degradation.

Figure 4-118 shows the side and top views of the structure of a water molecule adsorbed on the $(2 \times 1)$ reconstructed surface. "The oxygen atom of the water molecule is at the top of a cadmium atom on the surface. 'The black balls represent cadmium atoms, and the grey balls represent tellurium atoms. In the 
top view, the large, medium, and small balls represent atoms of the first layer, second layer, and below the second layer, respectively. Adsorption of the water molecule causes rumpling of the $\mathrm{CdTe}(001)$ surface under the water molecule. The cadmium atom under the oxygen atom is pushed inward by $0.3 \AA$. The two tellurium atoms marked by two arrows are pushed downward slightly $(-0.05 \AA)$. 'The $\mathrm{H}-\mathrm{O}-\mathrm{H}$ bond angle is $106.8^{\circ}$, about $3^{\circ}$ larger than that of a free water molecule (103.9"). The O-H bond length is $0.96 \AA$, about the same as that of a free water molecule. The cadmium-oxygen distance is $2.5 \AA$. The calculated adsorption energy is $0.22 \mathrm{eV}$, indicating that the adsorption could be just molecular, a conclusion that is confirmed from the charge-density plot of the oxygen-cadmium-tellurium plane. We further found that the adsorption of water molecules on the $\mathrm{CdTe}(001)$ surface can only be molecular, not dissociative.

We found that the formation of cadmium and tellurium vacancies did not significantly change the adsorption energy of a molecule on the $\mathrm{CdTe}(0010)$ surface. Figure 4-119 shows the side and top views of the structure of a water molecule adsorbed on the cadmium atom near the tellurium vacancy site on the (2x1) reconstructed surface. 'The adsorption energy was calculated to be $0.21 \mathrm{eV}$, which is comparable to the adsorption energy on the perfect surfaces.

We further used indium- and copper-doped CdTe to simulate the effects of electrons and holes, respectively, on the adsorption of water molecules on the $\mathrm{CdTe}(001)$ surface by substituting a cadmium atom, which is located five layers from the surface, with a copper atom or an indium atom in our calculations. However, no significant change on the adsorption energies was observed. Our results suggest that the first scenario-that water vapor may cause peeling of the front contact and oxidization of the back contact-is more likely the mechanism for moisture-induced degradation of a CdTe solar cell.

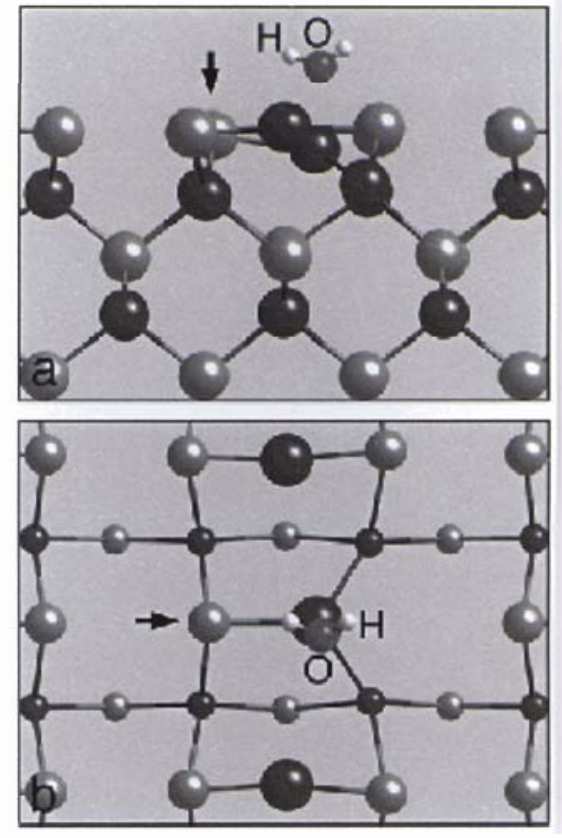

Figure 4-119. Side (a) and top (b) views of the structure of the adsorption of a water molecule on the cadmium atom near the tellurium vacancy site on the (2x1) reconstructed surface. 


\section{Multifluid Flow and Multicomponent Reactive Transport in Heterogeneous Subsurface Systems}

SB Yabusaki, (a) M D White, (a) DH Bacon, (a) VL Freedman, ${ }^{\text {(a) J M Malard, (a) M }}$ Rosing, (b) PC Lichtner, (c) JC Parker, ${ }^{(d)}$ E Park, ${ }^{(d)}$ CI Steefel, ${ }^{(e)}$ M L Rockhold, (a) and Y Fang(a)

(a) Pacific Northwest National Laboratory, Richland, Washington

(b) Self-Employed, Fort Collins, Colorado

(c) Los Alamos National Laboratory, Los Alamos, New Mexico

(d)Oak Ridge National Laboratory, Oak Ridge, Tennessee

(e) Lawrence Livermore National Laboratory, Livermore, California

This research has resulted in development of new capabilities that advance the understanding of processes and properties controlling contaminant behavior in complex environments in the subsurface. New science that expands the robustness and range of subsurface process modeling has been included in our simulations. Advanced parallel programming tools, utilities, and interfaces have been designed and implemented to increase usability, computational performance, and efficiency of the simulation software used in this research. 'These accomplishments were achieved with 416,689 central processing unit hours of computation on EMSL's Linux cluster supercomputing system.

\section{Radionuclide Migration at the Nevada Test Site}

The parallel code PFLOTRAN for modeling reactive flows in porous media was developed by Peter Lichtner and tested on EMSL's supercomputer. PFLOTRAN is based on the Portable Extensible Toolkit for Scientific computation (PETSc) parallel libraries developed at Argonne National Laboratory by Balay and co-workers (2004).

Preliminary results for a field-scale problem describing radionuclide migration from the BENHAM underground test at the Nevada Test Site were obtained using EMSL's supercomputer. A relatively small three-dimensional problem with 926,250 degrees of freedom was run on the system twice, once using 64 processors and then using 128 processors. Excellent scaling was obtained with run times of 50 and 25 minutes, respectively. Results based on an effective porous medium model suggested that plutonium moved more rapidly compared to other radionuclides based on fit-to-breakthrough data collected from a limited set of observations from nearby groundwater monitoring wells. 


\section{Multifluid Subsurface Flow and Transport}

\section{$\mathrm{CO}_{2}$ Injection at the Mountaineer Power Plant Site}

The parallel Fortran 90 version of STOMP, a scalable, parallel-processingimplementation of the multifluid subsurface flow and transport simulator, was developed by the PNNL for investigating carbon dioxide injection, redistribution, and sequestration in deep geologic reservoirs. It was used for a pilot study at American Electric Power's Mountaineer Power Plant in New Haven, West Virginia (White et al. 2004a). The comprehensive and detailed numerical simulations will be used to support integrated risk assessments and design guidance for injection strategies, protocols, and permits for a demonstration project for carbon dioxide injection in these deep saline aquifers.

\section{Residual Nonaqueous Phase Liguid Migration Processes}

High-resolution STOMP90 simulations were used to investigate the historical migration of carbon tetrachloride at the Hanford Site 200 West Area in Richland, Washington, during the disposal period (beginning in 1954) to date, including the soil vapor extraction activities (Figure 4-120), and to predict the current distribution and fate. This work successfully demonstrated a scientifically defensible scalable simulator for three-phase, three-component, aqueous-nonaqueous phase liquid-gas systems undergoing phase transitions (White et al. 2004b).

\section{Coupled Multifluid Flow and Reactive Transport}

The parallel processing CRUNCH multicomponent reactive transport simulator is a joint development between Lawrence Berkeley National Laboratory and PNNL and has a variety of attributes that make it ideal for investigating movement of different contaminants simultaneously in complex geochemical environments (Fang et al. 2004).

This simulator was combined with the STOMP simulator to model the migration of high-ionic strength waste from a historical leak beneath the SX-Tank Farm at the Hanford Site. A simulation using 1 million grid cells with 29 chemical species predicted the formation of an uncommon mineral, cancrinite, which has been observed to form at elevated temperature (Figure 4-121).

\section{Unsaturated Flow and Multicomponent Strontium Ion Exchange}

At the Army Loop Road Vadose Zone Test Site in the Hanford Site 200 East Area, a combined CRUNCH/STOMP simulation (Figure 4-122) was used to study strontium ion exchange in the soil zone above the water table (i.e., the vadose zone).
Figure 4-120. Simulation of carbon tetrachloride migration and remediation beneath the 216-Z-9 trench on the Hanford Site using soil vapor extraction.

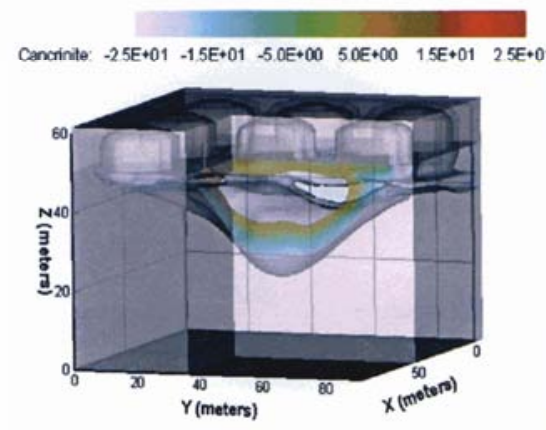

Figure 4-121. Predicted cancrinite concentrations. 


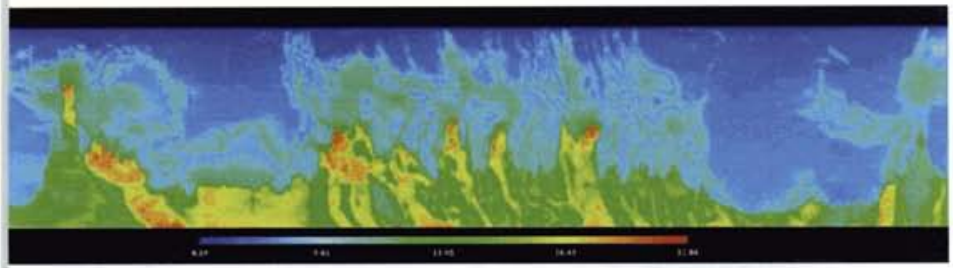

Figure 4-122. Calcium ion exchange sites after six days of infiltration of high strontium and magnesium solution (equivalents per cubic meter). Red and yellow represent higher calcium concentrations, and blue and green represent lower concentrations.

\section{New PETSc Algorithm}

PNNL's implementation of the block Krylov solver, developed by PNNL for subsurface multifluid modeling under this Computational Grand Challenge project, was recently adopted by the PETSc.

Citations

Balay A, K Buschelman, V Eijkhout, W D Gropp, D Kaushik, MG Knepley, L Curfman-McInnes, BF Smith, and H Zhang. 2004. "PETSc Users Manual" ANL95/11 - Revision 2.1.5. Argonne National Laboratory, Argonne, Illinois.

Fang Y, SB Yabusaki, and G Yeh. 2004. "A Generic Reaction-based Biogeochemical Simulator." In Computational Methods in Water Resources XV International Conference Proceedings, Elsevier, Chapel Hill, North Carolina.

White MD, N Gupta, ME Kelley, and JR Sminchak. 2004a. "Assessment of $\mathrm{CO}_{2}$ Injection and Monitoring Strategies at the Mountaineer Power Plan Site Using Scalable Numerical Simulation." In GHGT-7, 7th InternationalConference on Greenhouse Gas Control Technologies Proceedings, Pergamon Press, Vancouver, British Columbia, Canada.

White, MD, M Oostrom, and RJ Lenhard. 2004b. "A Practical Model for Mobile, Residual, and Entrapped NAPL in Porous Media." Ground Water 42(5):734-746.

\section{A Computational Approach to Understanding Oxidant Chemistry and Aerosol Formation in the Troposphere}

BC Garrett, (a) M Dupuis, (a) DA Dixon, (a) LX Dang, (a) SM Kathmann, (a) GK Schenter, ${ }^{(a)}$ SS Xantheas, ${ }^{(a)}$ JS Francisco, ${ }^{(b)}$ JT Hynes, ${ }^{(c)}$ A Morita, ${ }^{(c)}$ R Bianco, ${ }^{(c)}$ B Gertner, ${ }^{(c)}$ TJ Lee, ${ }^{(d)}$ KA Peterson, ${ }^{(e)}$ and JH Seinfeld ${ }^{(f)}$

(a) Pacific Northwest National Laboratory, Richland, Washington

(b) Purdue University, Lafayette, Indiana

(c) University of Colorado, Boulder, Colorado

(d) NASA Ames Research Center, Moffett Field, California

(e) Washington State University, Pullman, Washington

(f) California Institute of Technology, Pasadena, California 


\section{Dynamical Nucleation Theory}

The main goal of this task is the development of computational methods to calculate rates of vapor-to-liquid nucleation. In dynamical nucleation theory, nucleation is treated as a kinetic mechanism of cluster growth. 'The nucleation mechanism is assumed to be described adequately by simple condensation and evaporation of monomers from clusters

$$
A_{1}+A_{i-1} \stackrel{\beta_{i-1}}{\underset{a_{i}}{\leftrightarrows}} A_{i}
$$

where $\alpha_{i}$ is the evaporation rate constant for loss of a monomer from an imolecule cluster and $\boldsymbol{\beta}_{i-1}$ is the condensation rate constant for addition of a monomer to an $(i-1)$ molecule cluster. Dynamical nucleation theory consists of a new theoretical approach, based upon approximate molecular dynamics of the cluster for obtaining the kinetic parameters of the nucleation mechanism $\left(\boldsymbol{\alpha}_{\mathrm{i}}\right.$ and $\left.\boldsymbol{\beta}_{i-1}\right)$, followed by solution of the kinetic equations for cluster evolution. 'The emphasis of dynamical nucleation theory is the evaluation of monomer evaporation rate constants. Condensation rate constants are obtained from the evaporation rate constants by detailed balance, which requires calculation of equilibrium constants or populations of clusters in equilibrium with the monomer concentration. The cluster populations are determined from the absolute Helmholtz free energy of a given cluster is a fundamental quantity, as it determines the population of clusters in equilibrium with the monomer concentration. Using detailed balance, the ratio of the evaporation and condensation rate constants is related to differences in Helmholtz free energy between adjacent-sized clusters. This molecular approach entails three subtasks: 1) development of accurate interaction energies for molecules in the cluster needed for molecular simulations; 2) calculation of condensation and evaporation rate constants; and 3) calculation of the steady-state nucleation rate fromthe kinetic mechanism. 'The major focus of the work during the last year has been on the calculation of condensation and evaporation rate constants. 'The major computational expense in these rate constant calculations is determining Helmholtz free energies for forming i-molecule clusters that are constrained within spherical shells of fixed radii. Our initial calculations addressed nucleation in supersaturated water vapor, for which the nucleation is controlled by clusters comprised of up to 50 to 100 molecules. Molecular simulations of the Helmholtz free energies for these size clusters are computationally intensive and benefit greatly from access to EMSL's supercomputer. To perform these calculations, a new Monte Carlo simulation code was implemented in parallel during the first year of the project. During the second year, we have learned a significant amount about the extreme sensitivity of computed nucleation rates to the evaporation and condensation rate constants, and this knowledge has guided our further development of computational tools.

For small clusters, the rate constants for evaporation are typically much larger than those for condensation. 'Iherefore, the formation of larger clusters is unlikely, and cluster populations decrease as a function of the increasing cluster size in this size range. As the cluster grows, condensation becomes faster while 
evaporation decreases, until ultimately the two processes are approximately equal. The cluster size at which the forward and backward rates are about equal is called the critical nucleus. This is the cluster with the smallest population. Small changes in kinetic parameters can shift the critical cluster size and greatly alter the nucleation rate. Given the delicate nature of the nucleation process it is important to assess the effects of uncertainties in the kinetic parameters that govern the steady-state nucleation rate. Sensitivity analysis provides insight into the influence and identity of the dynamical quantities to which the nucleation rate, $\mathbf{J}$, is most sensitive. This, in turn, aids in the elucidation of specific reaction mechanisms and reduces computational effort by focusing only on those clusters that affect the nucleation rate. The sensitivity analysis can be performed in two ways. One, when the nucleation rate is expressed as a function of the evaporation and condensation rate constants $\left(\alpha_{i}\right.$ and $\left.\mathrm{Pi}\right), \mathbf{J}=\mathbf{J}(\alpha, \beta)$, then the nucleation rate has nearly unit sensitivity to all pre-critical clusters (e.g., a factor of two change in one of the kinetic parameters results in a factor of two change in the nucleation rate). Two, when the nucleation rate is expressed as a function of the condensation rate constants and equilibrium cluster populations $\left(\mathrm{N}_{i} \mathrm{EQ}\right)$, $\mathbf{J}=\mathbf{J}\left(\beta, N^{E Q}\right)$, then the sensitivity is peaked about the critical cluster. Thus, if the condensation rate constants and equilibrium cluster populations (or equivalently, the absolute Helmholtz free energies) of the clusters around the critical size can be determined directly, then the sensitivity of the nucleation rate on all the precritical clusters can be minimized. Calculations of equilibrium constants needed in the detailed balance expression require determination of the differences of Helmholtz free energies between adjacent clusters. Absolute free energy of the i-cluster can be obtained by accumulating free energy differences between adjacent clusters

$$
\mathrm{A}_{\mathrm{i}}=\Delta \mathrm{A}_{2,1}+\Delta \mathrm{A}_{3,2}+\ldots+\Delta \mathrm{A}_{\mathrm{i}_{\mathrm{i}_{-1}}}
$$

However, uncertainties for each free energy difference are also accumulated so the uncertainty in the absolute free energy Ai contains uncertainties from all precritical clusters. To minimize sensitivity to the parameters of the precritical clusters, we need a method to directly calculate the absolute free energy of an $i$ cluster, which computationally is much more demanding. The major focus of the second year has been the development of new computational tools, based upon the external work technique (Reinhardt and Hunter 1992), to directly calculate the absolute free energies of clusters near the critical cluster.

In the external work technique, an estimate of the absolute Helmholtz free energy is provided by determining of the external work, W. The change in internal energy of a system $\mathrm{AE}$ for a given process is given by

$$
\Delta \mathrm{E}=\mathrm{Q}+\mathrm{W}
$$

where $Q$ is the heat absorbed by the system. For a reversible process, the change in Helmholtz free energy of the system AA is equal to the work done on the system. For an irreversible process $\mathrm{W} \geq \mathrm{AA}$. For calculating the absolute free 
energy of an i-molecule water cluster, we calculate the work for the process of "turning on" the interaction potential between molecules in the cluster. The potential energy surface is slowly changed from noninteracting (an ideal gas state) to a fully interacting potential, the total heat released to the bath is accumulated during this process, and the change in internal energy $\Delta \mathrm{E}=\mathrm{E}$ (final) - $\mathrm{E}$ (initial) is calculated. 'The external work is then determined by $\mathrm{W}=\Delta \mathrm{E}-\mathrm{Q}$, which provide an upper bound to the free energy difference between the fully interacting and ideal gas states. The absolute free energy of the ideal gas state is obtained from an analytical expression, thereby allowing determination of the absolute free energy of the interacting state. A lower bound to the free energy is obtained by reversing the process (starting with the interacting state and slowly changing to the ideal gas state). A Monte Carlo code was developed to perform external work calculations on water clusters. By using many different starting configurations a thorough sampling of the (nearly) reversible paths, which join the two thermodynamic states of interest, can be achieved.

\section{Theory of Sum Frequency Generation Spectroscopy for Surfaces Important for Heterogeneous Reactions of Atmospheric Significance}

'The microscopic structures of the surfaces-both liquid and solid-that serve as reaction sites for many heterogeneous reactions of atmospheric significance are currently very poorly understood, which severely retards the understanding of the reactions themselves. Since these surfaces are generally believed to be quite complex, a very important potential guide for the ultimate performance of theoretical calculations for reactions on these surfaces would be insights on the surface structure provided by experimental surfacesensitive spectroscopy. In particular, the surface-sensitivesum frequency generation nonlinear optical spectroscopy technique, which allows the examination of a surface vibrational spectrum, is an especiallyimportant tool for the study of liquid-vapor interfaces, where many high-vacuum techniques suitable for solid surfaces cannot be employed; in view of its detailed vibrational character, it holds high promise for solid surfaces as well. On the other hand, connecting an observed sum frequency generation signal to the underlying molecular structure is not at all simple, and itself presents a major theoretical/computational challenge that must be met before the technique can provide the desired molecular-level view of the surface structure.

We have begun the construction of a theory for sum frequency generation by developing a first generation theoretical description of the sum frequency generation spectrum that includes the requisite microscopic hyperpolarizability tensors and their dependence on molecular vibrations, for the water surface, using a combination of electronic structure calculations and molecular dynamics simulations. Direct simulation of the vibrations was finessed via a separate theoretical analysis of the frequency shifts, dipole moment, and polarizability vibrational coordinate derivatives, etc. We also made certain simplifying approximations. Analysis of the resulting sum frequency generation spectrum of water, which is in overall good agreement with experimental results, established the (different) degree of surface sensitivity for different $\mathrm{OH}$ stretching vibration 
regions, and also revealed a number of surprising microscopic features. For example the dangling bond. $\mathrm{OH}$ portion of the spectrum-usually thought of as arising exclusively from water $\mathrm{OH}$ bonds perpendicular to the surface, and hence free of any hydrogen bonding to the hydrogen-in fact has considerable contribution from $\mathrm{OH}$ bond configurations, which are significantly tilted from the surface normal.

Despite this considerable success, it is clear that further improvements are definitely required. 'Thus, application of the theory to a water/methanol mixture, where the surface structure is already well characterized, succeeds only qualitatively in reproducing the observed behavior of the sum frequency generation $\mathrm{OH}$ dangling bond signal.

\section{List of Significant Methods/Routines or Codes Developed}

\section{Metropolis Monte Carlo simulation of cluster Helmboltz free energies}

A Monte Carlo code was written to perform external work calculations on water clusters and was adapted to run on any number of nodes on EMSL's supercomputer. 'The Monte Carlo code uses intrinsic commands that specify which node it is on. Given this node number, an initial random configuration of water molecules is generated. Jobs are submitted to the supercomputer using a queuing system, which requires a batch script. 'The batch script specifies the following: the total number of nodes to be used, to what directories to write the output, how much time will be required for job completion, from what account to charge the computer time, on what nodes to place the executables, and to erase all files on each node after completion. 'The resulting data sets are combined and averaged. By using many different starting configurations, a thorough sampling of the reversible paths which join the two thermodynamic states of interest can be achieved. For a 40-water-molecule cluster with 10 initial starting configurations run for 3 million Monte Carlo steps at $\mathrm{T}=243 \mathrm{~K}$, the Helmholtz free energy difference is $\mathbf{A A}=\mathbf{A}-A^{\text {ideal }}=(-146.7 \pm 3.1) \mathrm{kcal} / \mathrm{mol}$.

Citation

Reinhardt WP, and JE Hunter, III. 1992. "Variational Path Optimization and Upper and Lower Bounds to Free Energy Changes via Finite Time Minimization of External Work." Journal of Chemical Physics 97(2):1599-1601. 


\subsection{7}

\section{Instrument Development Laboratory}

The Instrument Development Laboratory designs, builds, and deploys advanced state-of-the-art instrument systems and custom application software in support of the ongoing experimental research efforts at EMSL. Staff in this laboratory support EMSL researchers and users by providing a wide variety of design and fabrication services for hardware and software, custom engineered solutions to research problems, and in-depth experience in the interface and control of commercial instrumentation. Because most EMSL user projects have unique needs, the Instrument Development Laboratory staff are especially skilled in the integration of commercial and custom hardware/software packages to suit the exact specifications of specific research projects.

\section{Representative Activities}

Instrument Development Laboratory staff provide support in real-time data acquisition and instrument control, as well as data visualization and management. They have experience in a variety of programming languages, including Visual Basic, Java, C, C++, LabView, Assembly, Access, and SQL Server. In addition, software staff are familiar with a number of instrument control strategies, including GPIB, Serial (RS-232), IR, TCP/IP, Analog and Digital I/O, and high-speed event counting and timing. Some of the Instrument Development Laboratory's most recognizable expertise and capabilities fall into the following areas:

- high-voltage electronics

- radio frequency electronics

- high-speed analog and digital systems

- digital signal-processing and field-programmablegate array technologies

- databases

- laboratory automation

- data acquisition

- instrument control

- common communications methodologies

- software design and implementation (.NET, C, C++, Visual Basic, JAVA)

- embedded systems and personal digital assistants.

The Instrument Development Laboratory Design Laboratory offers a staffed electronics and fabrication shop for EMSL research staff and facility users.

Customers will find a fully stocked parts supply, electronic components and small hardware, test and measurement equipment available for checkout, and ready assistance during business hours. For immediate hardware assistance, customers 
may access and receive assistance from staff at the Instrument Development Laboratory electronics laboratory. For work that is limited in scope and not time consuming, there usually is no charge to EMSL staff for services. For larger projects, staff may also access the electronics laboratory and speak with a staff member, who will happily assist the customer in defining the work to be performed and begin the process of designing solutions.

Custom software design, development, and support are critical services offered to customers of the Instrument Development Laboratory. Staff members specialize in several key services related to research: data acquisition, instrument control, laboratory automation, systems integration, data analysis and visualization, data management and archiving, and handheld and embedded systems. Using a modular code design model as their basis, the laboratory's software developers can efficiently develop software in a number of languages, tools, and scripts (e.g., Visual Basic, Java, C, C++, Labview, Assembly, Access, and SQL Server). In addition, software developers are skilled in a number of instrument control strategies, including GPIB, Serial (RS-232), IR, TCP/IP, Analog and Digital $\mathrm{I} / \mathrm{O}$, and high-speed event counting and timing.

The Instrument Development Laboratory Support Queue is accessible by customers via email (idlsupport@emsl.pnl.gov). The queue is monitored daily, and customers are encouraged to submit their requests for any type of service offered by laboratory staff. 'The Instrument Development Laboratory website (http://idl.emsl.pnl.gov) provides a full description of capabilities, access to the support queue, team highlights, recent projects, statement-of-work access, and a downloadable business plan.

Technical Support is readily available to assist research by providing software modifications, troubleshooting, equipment fabrication, and research instrumentation support.

\section{Capabilities}

- Electronics shop, workstations, tools, and user/staff assistance

- Parts and supplies, electronic components, and small hardware

- Test equipment checkout

- Custom software design, development, and support

- Data acquisition, instrument control, laboratory automation, and systems integration

- Data analysis and visualization, data management, and archiving

- Handheld and embedded systems

- Software modifications and bug fixes

- Research instrumentation fabrication and modification

- Electronics hardware design

- Control and monitoring software development 
- Data management and automated data analysis

- Systems engineering

- Embedded control

\section{Instrument Development Laboratory Staff}

\section{Daniel J. Gaspar, \\ Acting Technical Lead \\ (509) 376-2413 \\ daniel.gaspar@pnl.gov}

Kenneth J. Auberry

Senior Research Scientist (509) 376-1453

kenneth.auberry@pnl.gov

Michael A. Buschbach

Scientist

(509) 376-7207

michael.buschbach@pnl.gov

Eric Y. Choi

Scientist

(509) 376-4509

eric.choi@pnl.gov

James L. Eick

Technician

(509) 376-4540

james.eick@pnl.gov

James C. Follansbee

Research Scientist

(509) 376-4689

james.follansbe@pnl.gov

Navdeep Jaitly

Senior Research Scientist

(509) 376-6160

navdeep.jaitly@pnl.gov

Brian LaMarche

LTE Student

(509) 376-2127

brian.lamarche@pnl.gov
David C. Prior

Technologist

(509) 376-3923

david.prior@pnl.gov

Samuel O. Purvine

Senior Research Scientist (509) 376-3013

Samuel.purvine@pnl.gov

Thomas A. Seim

Senior Research Scientist (509) 376-2533

thomas.seim@pnl.gov

Kerry D. Steele

Chief Engineer

(509) 375-2880

kerry@pnl.gov

Kenneth R. Swanson

Senior Research Scientist

(509)376-0826

kenneth.swanson@pnl.gov

Beverley K. Taylor

Technician

(509) 376-5095

beverley.taylor@pnl.gov

Nikola Tolic

Senior Research Scientist

(509) 376-3090

nikola.tolich@pnl.gov 


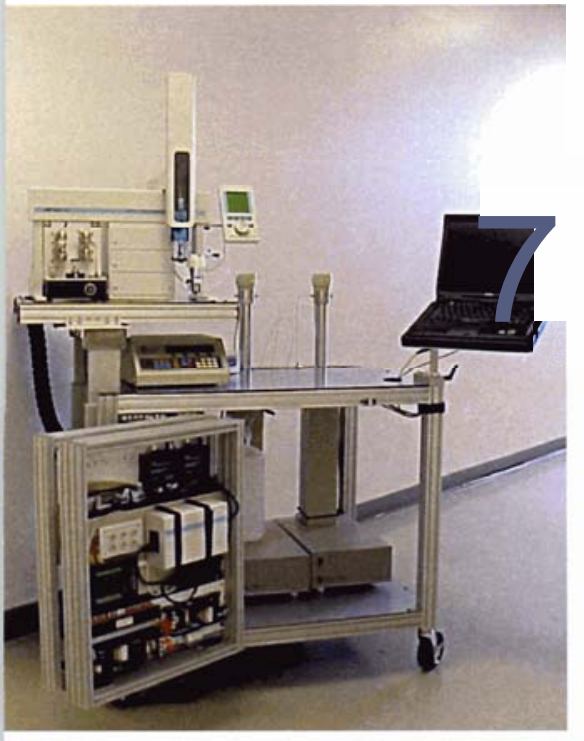

Figure 4-123. Automated liquid chromatography cart.

\section{Key Staff}

Key staff, besides those listed above, include David A. Clark, Marie C. Gibbons, Gary R. Kiebel, and Elena S. Peterson.

\section{Liquid Chromatography/Mass Spectrometry Automation - Enhanced Throughput and Reproducibility}

\author{
M A Buschbach, ${ }^{(a)}$ DF Hopkins, ${ }^{(a)}$ E A Livesay, ${ }^{(b)}$ DC Prior, ${ }^{(a)}$ CC Langley, ${ }^{(b)}$ \\ BK Taylor, ${ }^{(a)}$ K Swanson, ${ }^{(a)}$ and R Zhao ${ }^{(a)}$ \\ (a) W.R. Wiley Environmental Molecular Sciences Laboratory, Richland, \\ Washington \\ (b) Pacific Northwest National Laboratory, Richland, Washington
}

High-resolution mass spectrometry typically requires two or three hours of data collection per sample run, and an entire campaign devoted to the study of a single organism may include hundreds or thousands such sample runs in order to produce conclusive results. Ideally, data collection should be as reproducible as possible, minimizing unwanted variances which may impact the results. Automated sample handling and data acquisition provide numerous advantages in reducing slight procedural variations can lead to less reproducible results.

We have worked closely with the scientists at EMSL's High-Performance Mass Spectrometry Facility to develop the automated liquid chromatography cart (Figure 4-123) to enhance the throughput of samples on the FTICR and ion trap mass spectrometers. For versatility and convenience, different valve and pump configurations can be set up on different carts. The mobile cart configuration makes it extremely convenient for researchers to move a liquid chromatography setup from one mass spectrometer to another instrument, or a sample from one cart configuration to another cart. The automation project has produced a system which enables continuous ( 24 hours a day, seven days a week) unattended operation, while minimizing procedural variations.

Furthermore, we automated the process of liquid chromatography/mass spectrometry sample delivery and data acquisition through the use of custom software. Another generation of automation software has recently been implemented, enhancing reliability and adding increased flexibility for future improvements. The overhaul to the software established two fundamental differences between the original and the revision.

First, where previously the timing and logic of the sample delivery process were coded into the software with a state machine, this information is now carried within an easily modifiable script and executed using a scripting engine. The scripting engine executes each script instruction in succession, giving the 
operator a reference for proper operation. Adjusting the sample delivery process no longer requires the involvement of an engineer, as it can be accomplished with the script editor included with the software.

Second, creating a fluidics cart configuration for use in the liquid chromatography/mass spectrometry system is now a visual process (Figure 4-124). Operators and researchers select a representation of each fluidics component (pump, valve, mixer, etc.) in the software, place it on the liquid chromatography/mass spectrometry fluidics design window, and then route the components together with visible connections that represent fluidics tubing. Components themselves are given the communication properties necessary for interfacing with the physical device they represent. Once this is done, operators can use this visual design to alter the state of the components at will. For instance, an operator can shift the position of a valve, run a pump, or move a column simply by using

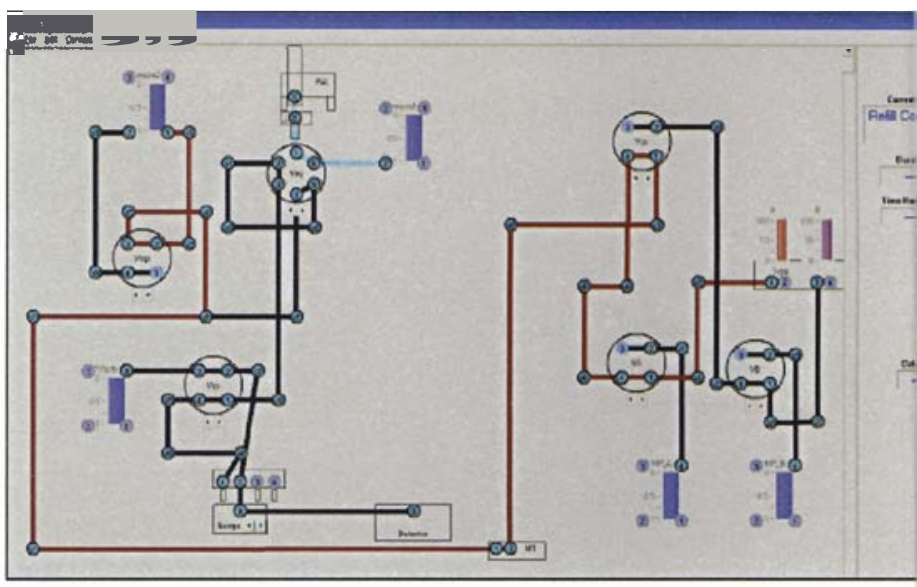
the software component interface in the design window. In addition to easing the process of fluidics cart configuration, the visual representation will change in response to executed script instructions. Consequently, during a sample delivery the operator can monitor the status of the process by observing the various component states in the design window.

In addition to these foundational changes, Instrument Development Laboratory staff also developed a more consistent method for initiating a Bruker Mass Spectrometer acquisition. The Bruker acquisition software, XMass, often failed to start correctly at the outset of a sample run; this would result in the loss of the pending sample and often prevented the liquid chromatography/mass spectrometry sample queue from progressing further. The updated software continually restarts XMass until it has initialized correctly, then proceeds with the acquisition. A number of other usability enhancements were also added, including the ability to save sample queues for future use, the option to enable the usable columns for each sample run, and a consistent error handling mechanism, among others. Over time, various errors are discovered and addressed, and this has resulted in a substantial decrease in the error rate.

Instrument Development Laboratory staff have devoted considerable effort to produce an enhanced application that is consistent, easily configurable, and able to manage any reasonable configuration and sample delivery process that researchers can envision. With these updates to the liquid chromatography/mass spectrometry software, EMSL proteomics researchers have been provided the tools necessary to increase their efficiency and maximize sample throughput. 


\title{
Confocal Microscope with FPGA Processing
}

\author{
MS Resat, ${ }^{(a)}$ JC Solinsky, ${ }^{(a)}$ HS Wiley, ${ }^{(a)}$ KA Perrine, ${ }^{(a)}$ DF Hopkins, ${ }^{(b)}$ and \\ T A Seim ${ }^{(b)}$ \\ (a) Pacific Northwest National Laboratory, Richland, Washington \\ (b) W.R. Wiley Environmental Molecular Sciences Laboratory, Richland, \\ Washington
}

EMSL's high-speed confocal microscope can simultaneously acquire two color images at speeds up to 30 frames per second, providing the capability to perform near-real-timefluorescent resonance energy transfer and ratiometric analysis of confocal images. Confocal capabilities are necessary for three-dimension reconstruction and correlation of image slices with identified signaling and propagation chemistry, using observation of the fluorescence originating from spatially localized portions of cells. Such information is important not only for adding to the existing knowledge base related to cellular signaling in general, but it also provides primary data to critically test developing spatial models of intercellular signaling.

'The high-speed confocal microscope is designed to simultaneously capture output from two intensified charge-coupled diode cameras. Software provided by the Instrument Development Laboratory controls laser output from the microscope via an eight-channel acoustic-optic tunable filter. "This custom software also controls the $\mathrm{XY}$ axis of the microscope using a high-resolution Ludl stage, and controls the $\mathrm{Z}$-axis using a PT piezo focus drive. Images from the two cameras are acquired synchronously and merged using a software package developed at PNNL. To maximize the flexibility of the control software, this software package uses a text-based script engine that exposes the functionality of all hardware components of the system, allowing the operating scientist to build or customize $a d$ boc experiments.

The fundamental software/hardware infrastructure needed to conduct real-time fluorescent resonance energy transfer includes two cameras that are matched and aligned to the pixel level so that alternate frames are acquired using alternate excitation wavelengths, and also the ability to capture images from both cameras simultaneously. "The capability to match and fuse two images in real time is being developed using a field-programmablegate array as a reconfigurable processor. Additional manipulations include averaging of multiple images, co-adding images to create 16-bit pseudo-color images, and achieving a ratio of different images, with a correct registration of pixels. 


\section{A Control System for an Inexpensive and Configurable Ion Trap Mass Spectrometer}

DF Hopkins, ${ }^{(a)}$ ML Alexander, ${ }^{(b)}$ K Swanson, ${ }^{(a)}$ and M A Buschbach ${ }^{(a)}$

(a) W.R. Wiley Environmental Molecular Sciences Laboratory, Richland, Washington

(b) Pacific Northwest National Laboratory, Richland, Washington

'This project involved the development of an ion trap mass spectrometer that is both inexpensive and flexible, opening up the application of ion trap technology to a number of research areas. The primary thrust of this effort was to develop a basic hardware and software platform for ion trap mass spectrometry that will allow the construction and deployment of systems with capabilities tailored to the specific needs of a variety of scientific applications, with emphasis on the use of chemical ionization techniques to address the need for highly sensitive and specific measurements as well as analytical needs dictated by research areas such as atmospheric chemistry, catalysis, materials science, biology, and inhalation toxicology.

'This ion trap mass spectrometry platform can be used to develop and deploy systems for critical applications such as chemical weapons detection and nuclear proliferation monitoring. Although commercial systems are available, their hardware and software are tailored for specific needs and are not easily modified. Additionally, the cost of commercial systems $(\$ 20 \mathrm{~K}$ for the new ion trap mass spectrometry platform versus $\$ 60 \mathrm{~K}$ to $\$ 200 \mathrm{~K}$ for a commercial system) places them beyond the means of many academic researchers, particularly at smaller institutions. Thus, we have created a platform using inexpensive, commercially available computers, data acquisition systems, and control equipment combined with open source software for instrument control, data acquisition, and analysis. As such, the software can be readily tailored to specific applications, in contrast to commercial systems.

The hardware and software developed as part of this project enhance and extend the capabilities of two instruments used within EMSL: the proton transfer ion trap mass spectrometer and the laser desorption ion trap mass spectrometer. "The control system includes some components specific to the laser desorption ion trap mass spectrometer. system for the analysis of single biological and nonbiological particles, as an illustration of one application. 'The remainder of the system can be used by a wide range of ion trap mass spectrometry applications.

The control and acquisition hardware consists of four National Instruments PCI boards that provide the signal generation, timing, and data acquisition for the system. An optional component of the system is a field-programmable gate array-based particle discriminator. Developed in the Instrument Development Laboratory, this particle discriminator is used to trigger an ablation laser as a particle passes through its beam. 'The control software was written in Visual Basic 6.0 to take advantage of its Rapid Application Developmentenvironment and straightforward integration with the control hardware. 
The system described here was showcased at National Instruments NIWeek 2003 conference, where it was a finalist in the Lab Automation/ Research and Development category of the paper contest. It was also featured on the cover of Scientific Computing and Instrumentation Magazine, April 2004.

\section{Citation}

Hopkins, DF, ML Alexander, K Swanson, and MA Buschbach. 2004. "Control System for an Ion Trap Mass Spectrometer: Inexpensive and ConfigurablePlatform Can be Tailored to Specific Applications." Scientific Computing and Instrumentation Magazine (April 2004), http://www.scimag.com/.

\section{SprayCool Project}

T Seim, ${ }^{(a)}$ K Regimbal, (a) M. Khaleel, ${ }^{(b)}$ RS Studham, ${ }^{(c)}$ and ND Tenney ${ }^{(d)}$

(a) W.R. Wiley Environmental Molecular Sciences Laboratory, Richland, Washington

(b) Pacific Northwest National Laboratory, Richland, Washington

(c) Oak Ridge National Laboratory, Oak Ridge, Tennessee (formerly of PNNL)

(d) Isothermal Systems Research, Spokane, Washington

Researchers from EMSL, PNNL, and Isothermal Systems Research are collaborating on the design and evaluation of a novel cooling technologySprayCool (patented by the latter company) — on a portion of PNNL's supercomputer. 'This evaluation will be conducted to measure improvements in computational speed and system reliability through this improved equipment cooling.

Heat generation is one of the most critical limitations in high-performance computing, and electronics in general. Electronic circuits consume power and, hence, generate heat, in direct proportion to their clock speed. With clock speeds now well into the gigahertz range, performance is often limited not by semiconductor technology, but instead by how much heat can be removed. If the heat is not adequately removed, the circuit will be destroyed. This is analogous to an automobile engine that overheats under load and thus must slow down to avoid being permanently damaged. Next-generation microprocessors have potential power dissipation of more than 100 watts, well beyond the capacity of current air cooling technologies. 'This limitation is resolved by slowing the microprocessor, which in effect reduces its computing capacity, thus impacting the effective computational rate of PNNL's supercomputer.

SprayCool technology addresses this problem by replacing the typical cooling medium (moving air) with a fluorinert $\left(3 \mathrm{M}^{\mathrm{TM}}\right)$ liquid that is sprayed directly in a finely atomized stream on the component being cooled. 'The liquid is vaporized on contact, and the heat of vaporization is supplied by the component, effectively cooling it. "The vapor is removed from the cooling apparatus and sent to a heat 
exchanger outside of the equipment cabinet. The heat exchanger cools the vapor, returning it to liquid form for reuse. "The system is completely closed-looped, with none of the cooling liquid vented to the atmosphere. The SprayCool technology can potentially remove 500 times the heat of conventional forced air cooling methods.

\section{Quantifying Peptide Abundance Ratios}

\section{Goshe ${ }^{(a)}$}

(a) North Carolina State University, Raleigh, North Carolina

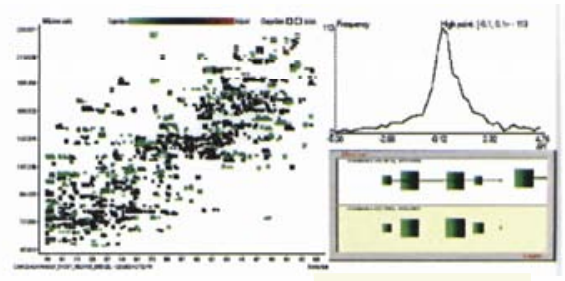

Figure 4-125. Complex datasets were searched for isotopic pairs and inspected using software developed by staff of the Instrument Development Laboratory.

This research focuses on analysis of yeast sample datasets to identify the changes occurring in protein abundance and the phosphorylation/glycosylation states of the detected proteins. Various methods using stable isotope labeling have been reported to examine global protein expression levels, but few have the ability to quantify and monitor post-translational modifications. A combination of metabolic labeling using ${ }^{14} \mathrm{~N} /{ }^{15} \mathrm{~N}$ isotopically enriched media and the phosphoprotein isotope-coded affinity tag approach using liquid chromatography MS/MS has been developed to identify and quantify phosphorylation and glycosylation for global proteomic studies.

Protein-identification and abundance-change analyses were performed using Instrument Development Laboratory-designed software (ICR-2LS and LaV2DG) (see the visualization example in Figure 4-125).

Datasets originating from previous collaborationswith the primary researcher were stored in the Proteomics Research Information and Management System. Dataset complexity and size required automated procedures for rapid processing and tools for inspecting and visualizing results. Original algorithms were developed and implemented in order to assist with data interpretation. Results of the analyses are expected to be published with contributors from the Instrument Development Laboratory listed as co-authors. 


\section{Digital Signal Processing with Field- Programmable Gate Arrays}

TA Seim, ${ }^{(a)}$ MA Buschbach, ${ }^{(a)}$ and EY Choi ${ }^{(a)}$
(a) W.R. Wiley Environmental Molecular Sciences Laboratory, Richland, Washington

Very large-scale digital circuits called field-programmable gate arrays (FPGAs) are being used to perform real-time signal processing of ultrasonic signals produced by chemical reactions.

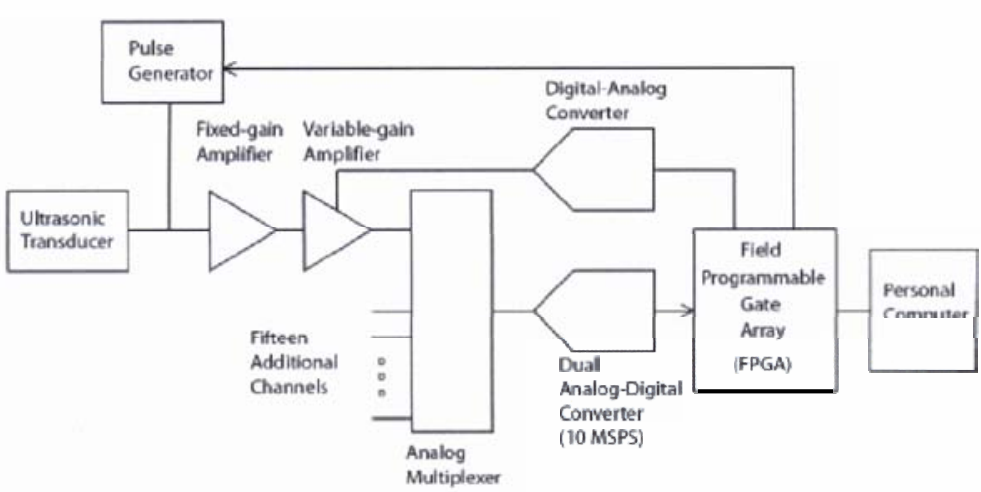

Figure 4-126. Block diagram of the data collection and analysis system based on FGPA technology. detect this emission.
'These arrays make it possible to move computations that were previously generated by computers down to the transducer level. The advantage of this approach is greatly accelerated signal processing, which means faster analysis of ultrasonic phenomena with higher precision and significantlylower measurement noise.

Photoacoustic spectroscopy is a new area of chemical analysis that offers the selectivity of absorbance spectroscopy at nearly the same sensitivity of reflectance spectroscopy. With this technique, the analyte is exposed to an intense pulse of noncoherent white light. 'The injection of this optical energy triggers a chemical reaction that yields an ultrasonic emission as a byproduct. An ultrasonic transducer is used to

The frequencies of ultrasonic signals can extend into the megahertz range, which requires digitizing rates of up to 10 times those frequencies. Computers have difficulty just transferring data at those rates, let alone performing any meaningful processing of the data.

FPGAs have two highly desirable traits; they have a very large logic density, containing up to 10-million logic gates, and they are field programmable. 'The very large logic density gives them the complexity to perform involved signal processing tasks such as digital filtering and pattern recognition. Field programmability permits this functionality to be rapidly and cost effectively applied to a diverse range of applications. 'The detection of very weak ultrasonic signals requires the repeated co-adding of many waveforms to increase the signal-to-noise ratio. An FPGA has internal random access memory that is used to store the co-added waveforms of each of its 16 transducers. Internal logic controls the number of samples per waveform, the sampling rate, and the number waveforms to co-add.

A novel self-calibration feature allows the coupling coefficient between the transducer and the medium to be measured for accurate quantitative measurements. When operated, the transducer is pulsed to turn it into a sound emitter or transmitter. When the pulse is stopped, the transducer becomes a receiver. "The emitted sound pulse reflects off the sample well and is detected by the same transducer that produced it. The pulse magnitude is a measure of the degree of acoustic coupling between the transducer and the sample, thereby allowing system calibration. 
The instrumentation package includes for each of its 16 transducers, in addition to the FPGA, an electronic pulse generator, a fixed-gain analog amplifier, a variable-gain amplifier, an analog multiplexer, and a high-speed (10-MHz) analog-digitalconverter. The FPGA communicates with a personal computer through the computer's printer port. 'The computer write capability to FPGA registers to control all aspects of data acquisition. A block diagram of the datacollection and analysis system is shown in Figure 4-126. A commercial FPGA development board was used as the foundation for this design. The applicationspecific interface electronics were developed on a custom electronics card that plugs into the development system, as shown in Figure 4-127. This design allowed rapid prototyping of the initial system.

\title{
Proteomics Research Information Storage and Management System
}

\author{
GA Anderson, ${ }^{(a)}$ GR Kiebel, ${ }^{(b)}$ KJ Auberry, ${ }^{(a)}$ D Clark, ${ }^{(b)}$ M E Powers, ${ }^{(b)}$ and \\ $\mathrm{N}$ Tolic ${ }^{(a)}$
}

(a) W.R. Wiley Environmental Molecular Sciences Laboratory, Richland, Washington

(b) Pacific Northwest National Laboratory, Richland, Washington

The Instrument Development Laboratory oversees and manages the Proteomics Research Information Storage and Management (PRISM) System, an essential part of the laboratory facility that is being used for proteomics research on a number of different organisms. 'This system manages the very large amounts of information that the facility generates and the data processing that it requires by collecting mass spectra data files directly from multiple mass spectrometers, managing the storage and tracking of these data files, and automating their processing into both intermediate results and final products. It also collects and maintains information about the biological samples used in research experiments and the laboratory protocols and procedures used to prepare them. Finally, this system allows users to readily locate and examine the information that it contains, and allows other information systems to access appropriate portions of it.

\section{Aerosol Sampler Controller}

T A Seim, ${ }^{(a)}$ M E Conley(a)

(a) W.R. Wiley Environmental Molecular Sciences Laboratory, Richland, Washington

The aerosol sampler is a self-contained portable instrument that acquires samples of airborne particles. "The heart of the sampler is a disk with $\mathbf{5 6 1}$ collection grids that capture the aerosol particles. Sample acquisition is fully programmable, including start time, sample time, sample mode and the number of samples. 
The aerosol sampler controller is an embedded microprocessor from Rabbit Semiconductor. 'This microprocessor, together with a custom-designed interface printed circuit board, provides motion and pump control of the rotational sampler assembly. An analog-to-digital converter monitors air mass flow. Motion control uses a 12-volt DC motor with an effective gear ratio of 17340:1. Rotational position is monitored with an incremental optical encoder. A Compaq Personal Digital Assistant (iPAQ) implements the operator interface. The controller initializes and receives data from a particle size counter through a serial RS-232 interface.

\section{Sensor System for Monitoring Tc-99 in Groundwater}

T A Seim, ${ }^{(a)}$ GA Anderson, ${ }^{(a)}$ DC Prior, ${ }^{(a)}$ ME Conley, ${ }^{(a)}$ T Harper, ${ }^{(a)}$ and MA Buschbach ${ }^{(\mathrm{a})}$

(a) W.R. Wiley Environmental Molecular Sciences Laboratory, Richland, Washington

With the new underground storage site for spent reactor fuel in Nevada, there is a great deal of concern for radioactive leakage into the groundwater. In order to monitor the amount of technetium-99 in the groundwater around the base of the mountain, a probe must be developed that would sample the groundwater and return the results to the surface.

Size requirements stated that the probe would be able to fit down a 4-inchdiameter well, which greatly limited the size of the hardware components that could be used. There are multiple pieces of hardware associated with this project. Each has a specific function within the process of sampling the groundwater. Most of the hardware that specifically deals with the sampling is concealed within a 3.5-foot by 4-inch Delron capsule. Within the capsule there are three photomultipliers; a flow cell; a Rabbit 3000 embedded processor with Ethernet on a custom fabricated board, which contains a digital to analog converter for high voltage control as well as a complex programmable logic device for counting light pulses from the photomultiplier tubes; two amplifier/discriminator boards; a high-voltage controller board; and all interconnect wiring. Outside of the probe capsule are the 15- and 24-volt power supplies that are mounted in a box along with a Sapphire stepper pump and its controller board. "There is also a wireless modem that will be used for hardware control from a dumb terminal as well as transmitting data. Because we are using a Rabbit 3000 as the processor for this project, all the code was written in $\mathrm{C}$ using National Semiconductor's Dynamic C editor and compiler.

'The software controls all hardware associated with the sampling of the ground water. The application is broken down into separate tasks. Using the Dynamic C's Micro OS-II, we are able to assign priorities to each of the tasks by using the operating system's pre-emptive multitasking features 


\subsection{8}

\section{Computing and Networking Services}

'The primary mission of EMSL's Computing and Networking Services Group is to provide the infrastructure and computing services within EMSL for an advanced computing environment that enables staff, visitors, and collaborators to effectively use computer and network resources for their scientific and business requirements. In supporting the growing business and research needs of EMSL in the area of information sciences, this group secures global information access to EMSL facilities by providing online remote access to both computing resources and scientific equipment. A large portion of the efforts undertaken by group staff involves providing customer support to EMSL researchers and offsite users. For offsite users, staff provide secure information access and dissemination among EMSL researchers and a global scientific user community.

\section{Representative Activities}

One of the primary roles of the Computing and Networking Services Group is to provide computer support to all EMSL users and visitors, including scientists, technicians, and support staff. Computing support includes computer procurement assistance, setup, delivery, connection, upgrade installation, and 24-hour computer emergency support. System administration services include configuration management, software upgrades, security standards, account setup, and automated backup. This group is responsible for the design and implementation of EMSL's computing infrastructure, software, and Internet application development and support; conference room support; and management of computer maintenance contracts.

Staff within the Computing and Networking Services Group are responsible for a variety of tasks related to EMSL's computing infrastructure, including:

- security

- remote access

- configuration control

- desktop computing

- scientific computing

- compute clusters

- wireless and wired network

- infrastructure design and upgrade

- web hosting and services

- software application development and deployment

- auditorium and conference room support 
- scientific user accounts and access control

- distributed file services and data management

- distributed service applications

- file and source code version control services

- database services

- personal digital assistants

- wireless devices.

\section{Capabilities}

- Design, install, configure, and maintain Windows, Macintosh, and Unix/ Linux hardware and software

- Manage office, laboratory, workgroup, and clustered configurations

- Maintain network system architectures

- Develop security and remote access methodologies

- Develop and manage distributed file systems

- Manage audio/visual hardware and software

- Develop software applications.

\section{Upgrades}

\section{EMSL Enclave}

To encourage open access while protecting PNNL from computational vulnerabilities, EMSL and PNNL implemented the "EMSL Enclave - a series of firewalls that "compartmentalize" EMSL's computer network from that of PNNL's and the open internet.

The enclave implementation provides the foundation for easier access to EMSL by creating the framework for differentiating EMSL's computer security requirements from PNNL's. 'The 2004 implementation of the EMSL Enclave was the result of a three-year effort involving multidisciplinary teams of staff from both EMSL and PNNL. Continued improvements to the architecture are planned in 2005.

\section{Collaboration Enclave}

The existing EMSL network is not positioned to use greater network bandwidth when it becomes available. In 2004, this project was started as a first step in enabling EMSL to use existing and future network bandwidth, a critical component for improving EMSL's collaboration and data-sharing capabilities. The increased bandwidth will be achieved by providing a high-performance collaborative network environment where a central firewall complex is not the main bandwidth-limiting factor to EMSL's connection with remote peers. 
PNNL has shown a great deal of interest in the success of this project, and is providing funding in 2005 to take the Collaboration Enclave from an exploratory project to a production network.

\section{Sygate Security Agent}

In 2005, Sygate's Security Agent software is being installed on all EMSL Windows desktop computers. The software provides key security features that detect malicious network attacks and protect the system from revealing information about itself. If rollout of this software to EMSL systems is successful, then the software will be installed on all PNNL systems as well.

\section{Computing and Networking Services Staff}

Linda M. Connell

Technical Lead

(509) 376-2375

linda.connell@pnl.gov

\section{Scott W. Campbell \\ IT Specialist}

(509) 376-2952

scott.campbell@pnl.gov

Timothy S. Carlson

Senior Research Scientist

(509) 376-3423

timothy.carlson@pnl.gov

Boyd L. Champion

IT Specialist

(509) 376-2287

boyd.champion@pnl.gov

Patricia M. (Shelly) Fangman

Research Scientist

(509) 376-5382

shelly@pnl.gov

Phillip P. Gackle

Senior Research Scientist

(509) 375-2130

philip.gackle@pnl.gov

Dan N. Hagedorn

Senior Research Scientist

(509) 376-8206

dan.hagedorn@pnl.gov
Tim J. Lawson

Research Scientist

(509) 376-2137

timothy.lawson@pnl.gov

Jeffrey Larsen

IT Specialist

(509) 376-1729

jeKlarsen@pnl.gov

'Thomas J. Mathieu

User Support Coordinator

Senior Research Scientist

(509) 376-9711

thomas.mathieu@pnl.gov

Nicholas A. Nanni

Technician

(509) 376-1714

nick.nanni@pnl.gov

Douglas J. Nordwall

Senior Research Scientist

(509) 376-4308

doug@pnl.gov

Shaun T. O'Leary

Senior Research Scientist

(509) 376-3505

shaun@pnl.gov

Andrew E. Rakowski

Senior Research Scientist

(509) 375-2238

landrew@pnl.gov 
Cheryl A. Hartwell

IT Specialist

(509) 376-2327

cheryl.hartwell@pnl.gov

Zoe C. Johns

Research Scientist

(509) 376-2038

zoe.johns@pnl.gov

Bryce P. Kaspar

IT Specialist

(509) 376-3949

bryce.kaspar@pnl.gov
Michael S. Russcher

I T Specialist

(509) 376-0300

Mike.Russcher@pnl.gov

Aaron I. Thronas

Research Scientist

(509) 376-1170

aaron.thronas@pnl.gov

Tyson A. Tucker

Research Scientist

(509) 372-6766

tyson@pnl.gov

Ralph S. Wescott

Senior Research Scientist

(509)372-6901

ralph.wescott@pnl.gov

\section{Key Staff}

Key staff, in addition to those listed above, include Gerald A. Funnel1 and Bruce J. Herrin. 


\subsection{9}

\section{User Services and Outreach}

'The User Services and Outreach Group promotes awareness of EMSL, leading to its increased usage and impact to science, and facilitates and documents this usage for the national user facility and its sponsors.

\section{Representative Activities}

'The User Services and Outreach Group plans, develops, and coordinates implementation of EMSL's outreach strategy to promote the awareness, reputation, and use of EMSL as a national user facility. 'Ihe group is one of the primary points of contact for PNNL and external media requests for general information. Outreach activities, which are undertaken to increase local, regional, and national awareness of the capabilities and accomplishments at EMSL, include:

- organizing the EMSL User Meeting and workshops

- providing tours of EMSL

- promoting the facility through local and offsite events

- creating print media, such as brochures, newsletters, posters, fliers, and photos

- developing and maintaining electronic media and websites.

\section{Capabilities}

'Ihe User Services and Outreach Group provides support services to facilitate and promote the effective use of EMSL resources by staff and users. It oversees those systems that enable users to submit proposals for research and staff to track the users' proposals, and also provides reports to PNNL, DOE, and federal and state regulators to document the success of EMSL as a national user facility. Activities include:

- coordinating production of the EMSL annual and monthly reports

- providing training for the EMSL User System

- providing access control for the facility

distributing proximity cards, computer passwords, and IDs to staff and users.

\section{Future Direction}

'This year, the User Services and Outreach Group will focused on building awareness of EMSL research capabilities and expertise. Examples of future activities include:

- identifying and establishing relationships with potential strategic users

- providing outreach to regional institutions to increase advocacy for the facility 
- attending national meetings

- reporting research accomplishments and products to sponsors.

\section{User Services and Outreach Staff}

Julia C. White

Technical Lead

(509) 376-1369

julia.white@pnl.gov

Jessica M. Foreman

Administrative Secretary

(509) 376-3412

jessica.foreman@pnl.gov
Amy R. Selch

Administrative Assistant

(509) 376-4452

amy.selch@pnl.gov

Pamela K. Stevens

Administrative Assistant

(509 372-2567

pam.stevens@pnl.gov

Gailann Thomas-Black

Senior Administrative Assistant

(509) 376-4366

gailann.thomas-black@pnl.gov

\section{Recent Outreach Activities Sponsored by EMSL}

In addition to participation in outreach activities, EMSL staff members initiate and sponsor various outreach events, many that pertain to education. Onsite activities range from small, one-day training sessions for undergraduates to 12-week sessions providing graduate-level lectures and hands-on experience with EMSL capabilities. Education outreach extends beyond EMSL walls as well. For example, this last year saw the first-ever adoption of EMSL-created science software by a university department for instruction in graduate courses.

More discussion on EMSL user outreach is provided in Section 5, EMSL user Outreach and Administration.

\section{Eastern Oregon University Students Learn Cbemical Sbifts}

In March 2003, Anna Cavinato of Eastern Oregon University in La Grande, and four undergraduate students (Richard Champion, Bina Hall, Dean Kuenzi, and Alicia McRoberts) visited for a one-day hands-on class to learn how to assign chemical shifts of several dipeptides using one-dimensional and twodimensional NMR on a 500-MHz NMR system in the High-Field Magnetic Resonance Facility. The students learned basic spectrometer operation skills and were given a perspective of how NMR is used to determine critical structural information of larger biomolecules. 'This class was taught by EMSL scientists David Hoyt and Joseph Ford, with additional instruction by PNNL postdoctoral fellow John Bagu and visiting EMS L user Peter Brzovic (University of

Washington, Seattle). A graphics presentation was also given by EMSL scientist Erich Vorpagel of the Molecular Science Computing Facility.

'The hands-on experience filled a gap in the Eastern Oregon University curriculum, which offered a course entitled "Instrumental Analysis Laboratory" 
but had no access to NMR instrumentation. The Eastern Oregon University Chemistry and Biochemistry Department is currently in the process of acquiring a 400-MHz NMR spectrometer. Installation and operation of this new instrument will in many ways be enabled by their experience at EMSL, both as students and as users.

\section{EMSL Software Deployed for First-Ever Educational Use}

For the first time, EMSL's Ecce software is being formally used in a classroom setting by an institution for educational purposes.

Matthew Asplund, Assistant Professor of Chemistry at Brigham Young University, persuaded the faculty of the Chemistry Department to forego their current chemistry software and implement Ecce —a problem-solving environment for computational chemistry — in their physical chemistry lab classes. About 100 chemistry and biochemistry majors benefited from the use of Ecce during the fall and winter semesters. Ecce allows Asplund's students to leverage the university's large supercomputer to perform higherlevel calculations than with their previous software - and the software's user friendliness and no cost served as added drivers for incorporating Ecce into Brigham Young's curriculum.

Ecce provides a sophisticated graphical user interface, scientific visualization tools, and the underlying data management framework that enable scientists to efficiently set up calculations and store, retrieve, and analyze data produced by computational chemistry studies. 'The software has been downloaded by more than 400 sites with multiple users at each site.

\section{Intensive Courses Introduce Students to Nanoscience, Nanotechnology, and EMSL Resources}

Two intensive nanoscience courses, developed with partial support from a National Science Foundation program through the University of Washington, have been offered at PNNL. A two-week course, Nanoclusters, Nanomaterials, and Nanotechnologies, was given in May 2003 and 2004 and is scheduled for 2005. A three-week course, Fabrication and Characterization $₫$ 'Nanomaterials, was given in January 2004 and 2005.

These courses, designed mostly for high-level undergraduate or beginning graduate students, involve both traditional classroom lectures and small project activities that provide students with hands-on introduction to several EMSL capabilities. The courses are coordinated by Fumio Ohuchi, University of Washington in Seattle, Lai Sheng Wang, Washington State University in the Tri-Cities, and Don Baer, PNNL. 'These courses provide a number of benefits:

- Reflecting current research paradigms, these courses are multidisciplinary and multi-institutional, offering students from a variety of backgrounds insight into how different disciplines contribute to new developments in nanoscience and nanotechnology.

- Subject experts introduce students to the topics and areas of current research. Course lecturers include leading researchers from PNNL as well as from

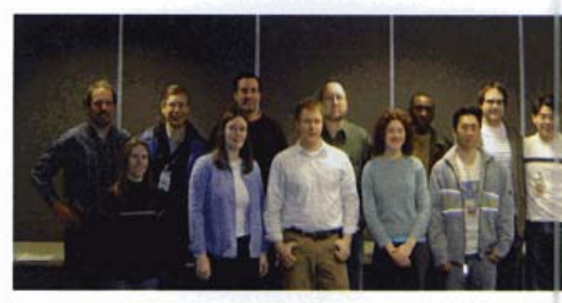

Students in the three-week course on Fabrication and Characterization of Nanomaterials, held at EMSL in January 2005. 


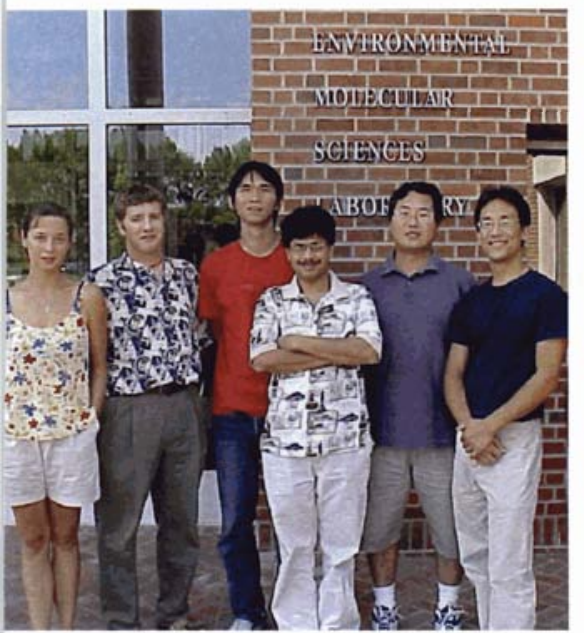

Six of the 2004 Summer Research Institute participants. the University of Idaho, University of Washington, and Washington State University. Special lectures have been given by faculty from Stanford University and the University of Alaska.

- Project activities are designed so that students receive introductions to many areas in nanotechnology. Most projects are small and question driven. Published papers and proposals for new externally funded collaborative research have evolved from these projects.

- In addition to the educational value for students, the interaction among students, faculty, and staff provide many formal and informal opportunities to share and develop collaborative research interests.

The project activities provide relatively in-depth training on several different EMSL capabilities, especially in surface fabrication and characterization methodologies (e.g., molecular beam epitaxy, ion accelerator studies, Auger electron spectroscopy, XPS, and HRTEM). More than 30 PNNL staff members (most of who work in EMSL) and university faculty are involved in the course.

Approximately 65 students from Alaska, California, Florida, Missouri, South Carolina, and South Dakota - as well as Idaho, Oregon, and Washington - have attended the four courses at PNNL.

\section{Summer Research Institute in Interfacial and Condensed Phase Chemical Physics}

PNNL, along with DOE, identified a DOE Office of Basic Energy Science Mission Stretch Goal: "To establish PNNL in a national leadership role in the area of theoretical and experimental condensed phase and interfacial chemical physics research." Part of achieving this goal was to establish the Summer Research Institute in Interfacial and Condensed Phase Chemical Physics in 2004. Steve Barlow, of EMSL's Chemistry and Physics of Complex Systems Facility team, is Director of the Institute.

'The Summer Research Institute provides access to highly qualified staff and stateof-the-art research facilities at EMSL. Research and mentoring tailored to each participant was designed to help educate young scientists to meet the demanding multidisciplinary challenges of the future science research environment.

'The Summer Research Institute is an advanced program that focuses on graduate students, postdoctoral staff, university faculty, and students entering graduate school. Projects are structured to maximize the participant's training and experience with advanced theoretical and experimental techniques. 'The Institute's goal is to bring outstanding young scientists to PNNL for a 10-week summer research and training experience in condensed phase chemical physics. This also provides an opportunity to establish long-term collaborations with the participants or their supervisors at their home institutions. Each participant works closely with a PNNL research mentor.

The Institute is advertised through brochures, the Summer Research Institute webpage (http://www.pnl.gov/si/), university contacts, EMSL users, and PNNL's Office of Fellowship Programs. Participants with a wide range of 
backgrounds are considered, including those in chemistry, physics, materials science, and related biological sciences.

Last year, approximately 25 candidates applied. Each was evaluated based on their application, letters of recommendation, proposed research, and resume. 'Ihe applicant and a PNNL research host jointly created a summer research project proposal, a critical part of the selection process. The summer research either became a part of a larger, existing program or it became a new collaborative effort between PNNL staff and faculty at the participant's home institution.

Ultimately, 14 PNNL scientists hosted 16 young scientists from 11 universities. Of the participants, 14 were graduate students, one was transitioning to graduate school, and one was a university faculty member. 'Ihese participants worked alongside scientists on research projects ranging from "Soft-Landing of Peptide Ions onto Self-Assembled Monolayer Surfaces" to "Chemical Characterization of Phosphorous Containing Soot Particles." The second year of the Institute begins in summer 2005. 


\section{2}

\section{EMSL Crosscutting Highlights}

Many scientific challenges addressed by research at EMSL involve use of multidisciplinary experts and approaches. One of EMSL's missions is to bring theoreticians with expertise in computer modeling of molecular processes together with experimentalists from the chemical, physical, and life sciences. While many of EMSL's user projects use resources required for the project within one of EMSL's six research facilities, several current and past research projects have benefited by use of resources from two or more facilities. As EMSL moves toward the future, it is the desire of the user facility to take full advantage of its problem-solvingenvironment by soliciting user research projects that "cross-cut ${ }^{\mathrm{n}}$ EMSL resources. "This section provides six examples of research projects at EMSL where resources were used from multiple facilities. These projects involved:

- NMR experiments and associated theoretical calculations for uranium and technetium compounds, which are critical to the development of remediation technologies

- characterization of gold clusters $\left(\mathrm{Au}_{20}\right)$ using XPS and $\boldsymbol{a} \boldsymbol{b}$ initio calculations; these materials may have unusual catalytic properties and potential use in nanoelectronics applications

- Examination of phosphorous containing soot particles collected from engine exhaust using a variety of spectroscopies and microscopies; this research may lead to an increased understanding of engine characteristics, additive chemistry, catalyst poisoning, and potential environmental effects

- Identification of a mineral never observed before as part of bacterial activity; this research has implications in elucidating the biogeochemical cycle of microbe-mineral interactions and enabling of bioremediation processes

- Synthesis of ceramic materials, nanomaterials, and composites and identification of their unique properties (e.g., extreme mechanical properties) using a variety of resources, including NMR, XRD, and TEM

- Studies of the chemistry and physics of sea salt particles, which affect health and climate.

The following research highlights provide more detail and illustrate how multiple facilities at EMSL are used to support critical research. 


\section{Reliable Electronic Structure Calculations for Heavy Element Chemistry: Molecules Containing Actinides, Lanthanides, and Transition Metals}

\author{
WA de Jong ${ }^{(\mathrm{a})}$ and $\mathrm{H} \mathrm{Cho}^{(\mathrm{b})}$ \\ (a) W .R. Wiley Environmental Molecular Sciences Laboratory, Richland, \\ Washington \\ (b) Pacific Northwest National Laboratory, Richland, Washington
}

In work by Cho et al. 2004, the temperature and isotope dependencies of the technetium-99 chemical shift were examined. Temperature dependencies are a result of the changes in vibrational motion of the molecular structure. The property and potential energy surfaces need to be calculated to determine the effect of the vibrational (and rotational) motion on the molecular properties and structure in solution at a finite temperature and for various isotopes. The results for the temperature dependence in pertechnetate are shown in Figure 4-128.

The calculated results show the correct trends but slightly underestimate the dependencies. This is caused by the dynamics of the molecularenvironment, and some rough calculations confirm this. The isotope dependencies were found to be in very close agreement with experimental data. Similar calculations were performed for the technetium-oxygen indirect spin-spin coupling values. The temperature dependence is much smaller, but calculations match very well with experimental data. The isotope dependence is too small to be measured, but it can be calculated. 'This isotope dependence and the combined isotopetemperature dependence have been calculated as predictions for future, more accurate NMR measurements.

In addition to the pertechnetate, the electric field gradient and the chemical shift tensor of the uranium-bonded ${ }^{17} \mathrm{O}$ (oxygen directly bonded to uranium) have been calculated for the experimental crystal structures of rutherfordine, a uranyl carbonate structure. The calculated NMR property tensors for ${ }^{17} \mathrm{O}$ in uranyl carbonate systems predicted a large chemical shift anisotropy of around 1500 ppm. This anisotropy will dominate the shape of the NMR spectrum of the ${ }^{17} \mathrm{O}$ NMR signal in uranyl carbonate crystals and produce a very broad NMR signal of around $1600 \mathrm{ppm}$. The predicted broad signal suggests that experimental measurement of ${ }^{17} \mathrm{O}$ in uranyl compounds requires a long sampling time at

EMSL's High-Field Magnetic Resonance Facility to perform experiments on rutherfordine. The experimentallyobtained spectrum is shown in Figure 4-129. This figure also contains the spectrum generated from calculated results. For these calculations the molecular structure in the left top portion of Figure 4-129 was used. 'There is already a reasonable agreement between theory and experiment,

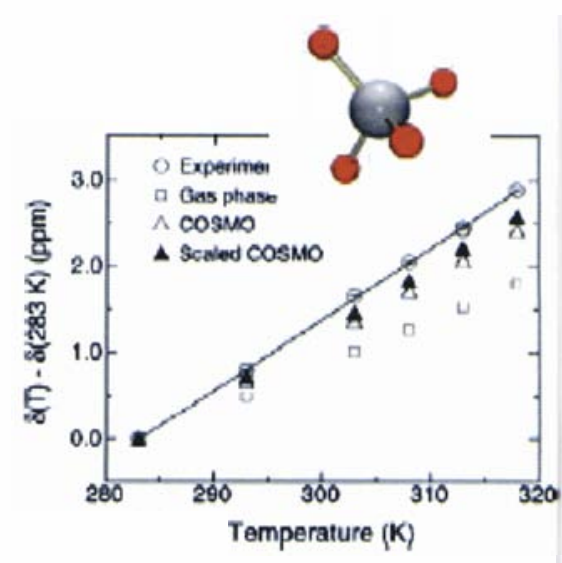

Figure 4-128. Theoretical versus experimental NMR shielding temperature dependence in pertechnetate of the technetium99 chemical shift.

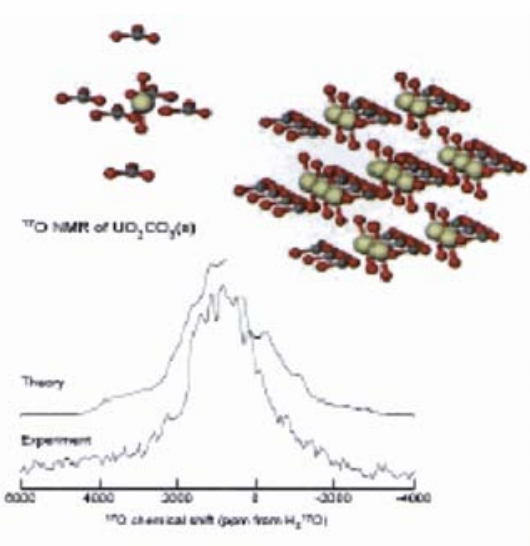

Figure 4-129. (Top, right) Rutherfordine, a uranyl carbonate structure. (Bottom) NMR spectrum of rutherfordine: theory versus experiment. 


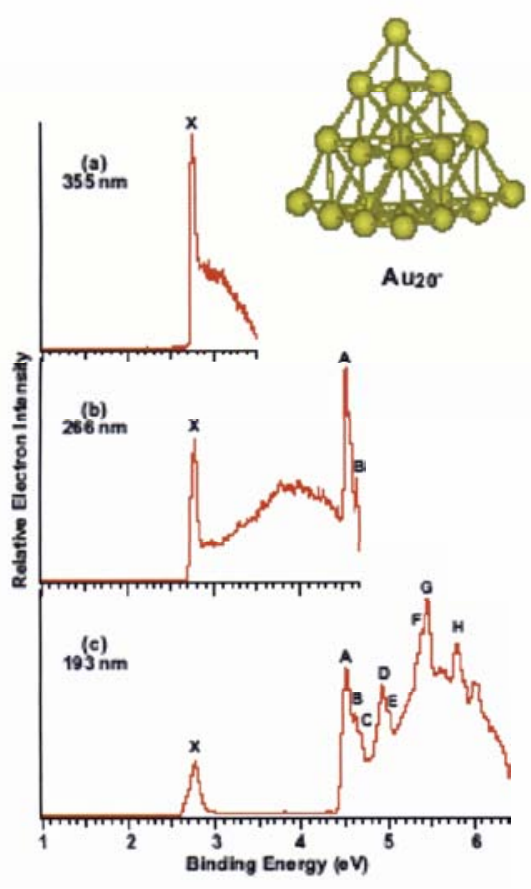

Figure 4-130. Photoelectron spectra of $A u_{20}-:$ (a) at $355 \mathrm{~nm}$ (3.496 eV), (b) at $266 \mathrm{~nm}(4.661$ $\mathrm{eV})$, and (c) at $193 \mathrm{~nm}(6.424 \mathrm{eV})$. Note the large gap in (c) between $\mathrm{X}$ and $\mathrm{A}$, indicating that $\mathrm{Au}_{20}$ is a closed shell molecule with a large highest occupied molecular orbital - lowest unoccupied molecular orbital gap. The inset shows the pyramidal structure of $\mathrm{Au}_{20}$. although some features are missing. To account for those features, the local environment around the central uranyl molecule needs to be accounted for in a more extensive manner. During the course of the project, calculations were started using a crystal structure model. This substantial calculation with 187 atoms (including 17 uranium atoms) is a true grand challenge calculation that will be continued next year.

\section{Citation}

Cho H, WA de Jong, BK McNamara, BM Rapko, and IE Burgeson. 2004.

"Temperature and Isotope Substitution Effects on the Structure and NMR the

Pertechnetate Ion in Water." Journal of the American Chemical Society 126(37):11583-11588.

\section{$\mathrm{Au}_{20}:$ A Tetrahedral Cluster}

$\mathrm{J} \mathrm{Li}^{,}{ }^{(a)} \mathrm{X} \mathrm{Li}{ }^{(b, c)} \mathrm{HJ}$ Zhai, ${ }^{(b, c)}$ and LS Wang ${ }^{(b, c)}$

(a) W.R. Wiley Environmental Molecular Sciences Laboratory, Richland, Washington

(b) Pacific Northwest National Laboratory, Richland, Washington

(c) Washington State University Tri-Cities, Richland, Washington

Small clusters often have different physical and chemical properties than their bulk counterparts. Materials assembled from finite-sized clusters have been intensively sought ever since the discovery of $\mathrm{C}_{60}$. One of the criteria that must be met for a cluster to be used as a potential building block for clusterassembled materials is its chemical stability relative to other reagents. A closed electron configuration with a large energy gap between the highest occupied molecular orbital and the lowest unoccupied molecular orbital is a prerequisite for the chemical stability of a cluster. Besides its high symmetry, the large highest occupied molecular orbital - lowest unoccupied molecular orbital gap of $\mathrm{C}_{60}$ is responsible for its chemical inertness and its ability to assemble into molecular crystals.

In this research, we describe a highly stable $\mathrm{Au}_{20}$ cluster discovered by combining photoelectron spectroscopic experiments and $a b$ initio calculations (Li et al. 2003). Photoelectron spectroscopy revealed that $\mathrm{Au}_{20}$ has an extremely large energy gap (Figure 4-131), even greater than that of $\mathrm{C}_{60}$, and an electron affinity comparable with that of $\mathrm{C}_{60}$. This observation suggests that the $\mathrm{Au}_{20}$ cluster should be highly stable and chemically inert. Using relativistic density functional calculations, we found that $\mathrm{Au}_{20}$ possesses a tetrahedral structure (inset in Figure 4-130), which is a fragment of the face-centered cubic lattice of bulk gold with a small structural relaxation. $\mathrm{Au}_{20}$ is thus a unique molecule with a similar atomic packing as the bulk, but with very different properties.

Each of the four faces of $\mathrm{Au}_{20}$ represents a (111) surface of fcc gold. It has a very high surface area (all the atoms are on the cluster surface), as well as a large fraction of corner sites with low coordination. The three different kinds of atoms 
in the $\boldsymbol{T} \boldsymbol{d}$ structure-four at the apexes, four at the center of each face, and 12 along the edges-have different coordination environments and may provide ideal surface sites to bind different molecules for catalysis (e.g., $\mathrm{CO}, 02$, and $\mathrm{CO}_{2}$ ).

\section{Chemical Characterization of Phosphorous Containing Soot Particles}

Z. Yang, ${ }^{(a)}$ A. Laskin, ${ }^{(b)}$ B.G. Bunting, ${ }^{(c)}$ J. P. Cowin, ${ }^{(b)}$ M.L. Engelhard, ${ }^{(b)}$ P.L. Gassman, ${ }^{(b)}$ M. J. Iedema, ${ }^{(b)}$ C. Wang, ${ }^{(b)}$ H. Wang, ${ }^{(a)}$

(a) University of Delaware, Newark, Delaware

(b) W.R. Wiley Environmental Molecular Sciences Laboratory, Richland, Washington

(c) National Transportation Research Center, Oak Ridge National Laboratory, Knoxville, Tennessee

Zinc dialkyldithiophosphate was used for more than 50 years in lubricating oil as antioxidant and antiwear additive. Engine oil can enter a combustion chamber through many routes: oil film formed on the cylinder liners' surface, pushing up effect by movement of pistons and rings, by blow-by gas, or from the valve guides and other components. Phosphorus had long been found to decrease the activity of automotive catalysts, affect the performance of oxygen sensors, and result in a degradation of emission control.

'This research examines the chemical properties of phosphorous containing soot, which may link engine characteristics, additive chemistry, and catalyst poisoning as well as environmental effects.

We collected soot samples in the exhaust of the Engine Test Stand at Oak Ridge National Laboratory at three different working conditions: varying consumption rate of lube oil doped with zinc dialkyldithiophosphate, varying load with zinc dialkyldithiophosphate doping, and varying load without zinc dialkyldithiophosphate doping. Here as a preliminary study, we focused on the study of soot in the series of varying load with zinc dialkyldithiophosphate doping. "The sampled was collected with Carbon Type-B T EM grid (Ted Pella, Inc.) by a time-resolved aerosol collector developed at EMSL. SEM, TEM, CCSEM/EDX, XPS, and Fourier transform infrared spectroscopy were used characterize the phosphorous containing soot. The advantage of the sampling technique with the TEM grid was that it fit with all of the above experimental techniques; more will be discussed later.

From the SEM images of soot samples of 2 percent, 29 percent, 57 percent, and 93 percent loads, a marked difference is noted in the particle morphology. 
At 2 percent load, unburned oil may be collected on the substrate. With increasing load, the morphology of soot particles changes from dispersive distribution to more aggregate distribution. TEM images provided more detail information about particles, showing that phosphorous containing soot particles changes from amorphous form into crystallized form, as seen in the images of particles of 2 percent and $\mathbf{9 3}$ percent loads.

With CCSEM/EDX, particle morphology changes can be quantified in aspect ratio and size distribution, and particle composition can be examined. From the CCSEM/EDX results, particle distribution is wider in the higher load case. In the ternary plots, the most evident change of particle composition is that the sulfur content reduced drastically from 2 percent to 93 percent loads. XPS data yield the chemical bond energy information of elements. From XPS results, the chemical states of phosphorous and zinc changed from 2 percent to 93 percent loads. Further experimental techniques such as mass spectroscopy are needed to identify the compounds that phosphorous and zinc formed in soot particles at different loads.

We used Fourier transform infrared resonance spectroscopy to study the hygroscopic properties of phosphorous containing soot particles. The sample grid was imaged by SEM before and after the spectroscopic study. By carefully selecting the secondary aperture on the Fourier transform infrared resonance microscopy, we were able to study the same cell on the TE M grid that had been imaged by SEM. The sample stage was kept at $23^{\circ} \mathrm{C}$ by a temperature controller. A dew point generator was used to supply water vapor of given relative humidity. Fourier transform infrared resonance spectroscopy results showed that for soot at different loads, there are marked differences in the water absorption spectrum when changing relative humidity and characteristic spectrum at zero relative humidity. However, the fresh phosphorous containing soot is generally hydrophobic.

The aging of phosphorous containing soot needs to be studied to understand overall environmental impact, since fresh soot released into atmosphere will be oxidized gradually during their lifetime in the air.

\section{Ferrous Hydroxy Carbonate is a Stable Transformation Product of Biogenic Magnetite} RK Kukkadapu, ${ }^{(a)}$ JM Zachara, ${ }^{(b)}$ JK Fredrickson, ${ }^{(b)}$ DW Kennedy,
AC Dohnalkova,

(a) W.R. Wiley Environmental Molecular Sciences Laboratory, Richland, Washington

(b) Pacific Northwest National Laboratory, Richland, Washington 
Dissimilatory iron-reducing bacteria catalyzes the reduction of iron (III) to iron (II) in soils, sediments, and subsurface sediments. A variety of biomineraliation products result from the interaction of dissimilatory iron-reducing bacteria with iron (111)-oxides, including fine-grained magneite under specific conditions (Kukkadapu et al. 2004). Although fine-grained magnetite is a frequently observed laboratory transformation product of iron (III) oxides by dissimilatory iron-reducing bacteria, there are few reports of biogenic, fine-grained magnetite in soils, sediments, and subsurface materials that have experienced in situ dissimilatory iron reduction. 'Ihe reasons for this absence in natural settings are unknown. Perhaps magnetite is unstable under iron-reducing conditions because of its small size or other properties resulting from biosynthesis.

Recently, we noted that the iron (II)/iron-total ratio of a fine-grained biogenic magnetite (approximately $10 \mathrm{~nm}$ ) produced by dissimilatory iron-reducing bacteria (Kukkadapu et al. 2004) was in excess (0.5 to 0.6) of stoichiometric magnetite (0.33). Iron (11)-excess magnetites or cation-excess magnetite of varying iron (II)/iron-total ratio ( 0.4 to 0.6 ) have been reported in the catalyst research area. Combustion-excess magnetites promoted complete reduction of carbon dioxide to carbon. 'The high reactivity of cation-excess magnetites for oxidized carbon compounds compared to stoichiometric magnetites, and its lack of persistence in natural settings where bacterial reduction has occurred, may result from its excess iron (II) or small particle size.

In this study, we followed the stability of a finegrained biogenic cationexcess magnetite in the presence of microbial oxidation products of lactate (carbonate and acetate). The initially bioproduced cation-excess magnetite slowly transformed (partially) with aging to ferrous hydroxy carbonate observed in TEM (Figure 4-131) and XRD (Figure 4-132). 'The XRD peaks and morphology (micron-size platy crystallites mixed with cation-excess magnetite) matched well with ferrous hydroxy carbonate (Erdos and Altorfer 1976).

Ferrous hydroxy carbonate grew at the expense of cation-excess magnetite. Approximately 50 percent of the cation-excess magnetite was transformed to ferrous hydroxyl carbonate during a 22-month period. Consistent with XRD and microscopy, a doublet due to an iron (II) mineral phase was observed in Mossbauer spectra (not shown). Reaction with carbonate and recrystallization to eliminate iron (111), or to enrich in the residual cation-excess magnetite, apparently led to the ferrous hydroxy carbonate structure. Combined Mossbauer and chemical measurements indicated a general decrease in the iron (II)/irontotal ratio of the residual cation-excess magnetite (to approximately 0.45 ) during the initial stages of ferrous hydroxy carbonate formation (21 to 28 days), implying recrystallization of a portion of the cation-excess magnetite.

Our findings indicated that ferrous hydroxy carbonate can result from remineralization of fine-grained, biogenic cation-excess magnetite from dissimilatory iron-reducing bacteria under anoxic conditions. 'Ihe apparent susceptibility of fine-grained biogenic cation-excess magnetite to transform to ferrous hydroxy carbonate may explain why the former phase is not more commonly observed in the sediments that have experienced iron (III) reduction. Whether biogenic cation-excess magnetite instability is promoted by its small, nanometer crystallite size or excess iron (II) remains undetermined: presumptive

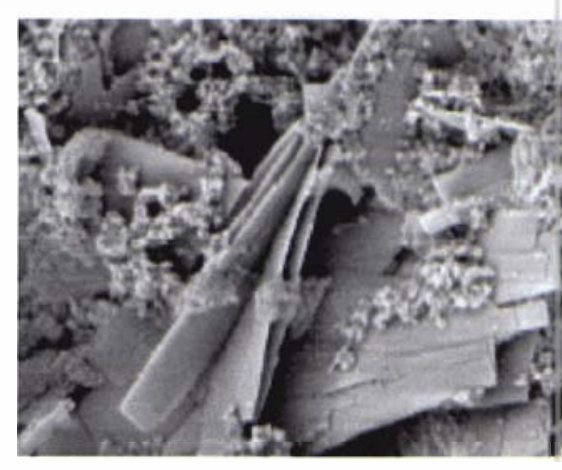

Figure 4-131. TEM image of a 22-month sample showing ferrous hydroxyl carbonate (micron-sized plates) and ground mass of magnetite.

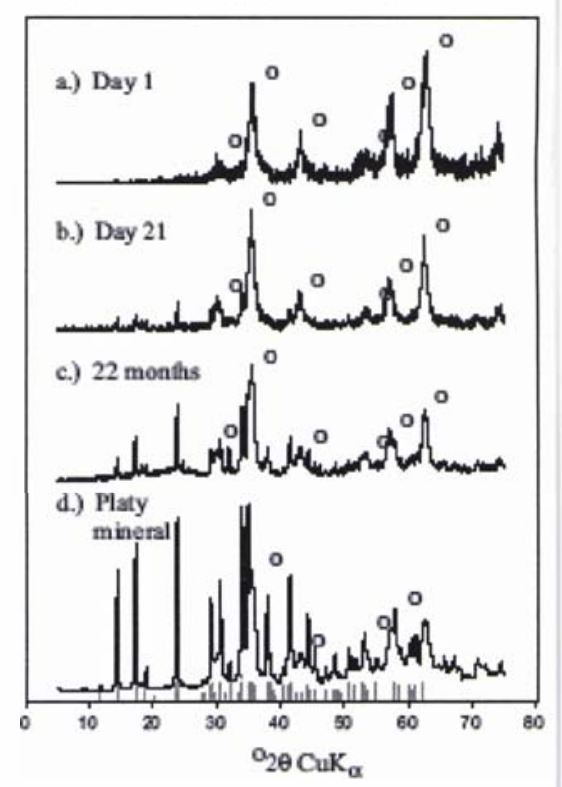

Figure 4-132. X-ray diffractograms: Transformation of cation-excess mannetite to ferrous hydroxy carbonate with time. Magnetite peaks are labeled by "o." Diffraction peaks for ferrous hydroxyl carbonate are shown in red. 
evidence suggests that excess iron (II) is important. Cation-excess magnetites are likely, however, to be unique products of topotactic magnetite formation by dissimilatory iron-reducing bacteria. In contrast, single-domain magnetite crystallites resulting from magnetotactic bacteria are larger (approximately 50 $\mathrm{nm}$ ) and stoichiometric in composition, and they appear more stable because they can persist for long periods as microfossils. The apparent instability of cationexcess magnetite to ferrous hydroxyl carbonate has important implications for magnetic stratigraphy, as our results show that a certain sequence of microbially mediated reactions may rapidly transform fine-grained magnetite from dissimilatory iron-reducing bacteria to a nonmagnetic iron (II) compound.

\title{
Citations
}

Erdos VE and H Altorfer. 1976. "Ein dem Malachit ahnliches basisches Eisenkarbonat als Korrosionsprodukt von Stahl." Werkstoffe und Korrosion 27(5):304-312.

Kukkadapu RK, JM Zachara,JK Fredrickson, and DW Kennedy. 2004.

"Biotransformation of 2-Line Silica-Ferrihydriteby a Dissimilatory Fe(II)-Reducing Bacterium: Formation of Carbonate Green Rust in the Presence of Phosphate."

Geochimica Cosmochimica Acta 68(13):2799-2814.

\section{Synthesis and Properties of Ceramic Materials and Nanomaterials/Composites}

\author{
L An, ${ }^{(a)}$ S Burton, ${ }^{(b)}$ J Ford, ${ }^{(b)}$ DE McCready, ${ }^{(b)}$ and C Wang, ${ }^{(b)}$ \\ (a) University of Central Florida, Orlando, Florida \\ (b) W.R. Wiley Environmental Molecular Sciences Laboratory, Richland, \\ Washington
}

Dr. An's research group at the University of Central Florida is involved in microfabrication of ceramics for micro-electro-mechanical systems applications, processing structure property relationships of ceramic materials, polymer-derived ceramics, synthesis and properties of nanomaterials and composites, as well as the mechanical behavior of materials. Dr. An's research has benefited from the use of multiple capabilities with the EMSL, including NMR, TEM, and XRD. Summarized below are highlights of three of An's research projects.

\section{Polymer-Ceramic Conversion of Liquid Polyaluminasilazanes for SiAICN Ceramics}

In contrast to traditional methods for the formation of ceramic precursors, An's group synthesized liquid-phased polyaluminasilazanes by reacting a liquid polyureasilazane with aluminum isopropoxide. This unique direct chemical-toceramic route has recently attracted attention since it possesses many advantages over conventional powder-based ceramic processing. For example, many unconventional structures, such as fibers, coatings, ceramic matrix composites and ceramic-based micro-electro-mechanical systems, can be easily fabricated by 
this technique. The direct chemical-to-ceramic technique also offers a unique opportunity to manipulate compositions and structures of ceramics at atomic/ nano levels through tailoring the chemistry of the precursors, thus, new materials with chosen properties could be designed and synthesized. Amorphous SiCN and $\mathrm{SiBCN}$ ceramics synthesized by this technique have shown unique thermal stability, creep resistance, and tunable electric properties.

Professor An's recent visits to EMSL's High-Field Magnetic Resonance Facility made use of a 300-MHz NMR spectrometer to analyze the aluminum and silicon coordination present within the materials following progressive processing conditions. Results revealing the structural evolution of polyaluminasilazanes during pyrolysis showed that the polymer-ceramic conversion can be classified into three stages: 1) cross-linking, 2) thermal decomposition, and 3) structural rearrangement. Measurements of the thermal mechanical properties influenced by the ceramic structural alterations are in progress and a manuscript detailing the above study has been submitted for publication (Dhamne 2005).

\section{Carbon-Nanotube-Reinforced Polymer-derived Ceramic Composites}

HRTEM was used to study the microstructure of carbon nanotube-reinforced polymer-derived ceramic composites. 'The research focused on 1) determining if the nanotubes can survive the processing and 2) observing the interface between the nanotubes and ceramic matrix.

The results (An et al. 2004) have clearly demonstrated the survivability of the nanotube through high-temperature processing and there is no chemical reaction between the nanotubes and ceramic matrix (clear interfaces). Figure 4-133 shows a typical HRTEM image of carbon nanotubes within the ceramic matrix.

\section{Synthesis, Characterization, and Optical Properties}

In this study, Wang et al. 2005 reported the synthesis of pristine, silicon-, and silicon/neodymium-doped YAG nanopowders via calcination of complex compounds of aluminum and yttrium with triethanolamine. 'The effects of doping and calcination temperature on phase composition, lattice parameter, and crystallite size were studied with $\mathrm{x}$-ray diffractometry and TEM. We found that silicon and silicon/neodymium doping have significant effects on crystallization and phase formation. The results showed that silicon doping can promote formation of YAG phase, and that single-phase silicon/neodymium:YAG can be synthesized at temperatures as low as $920^{\circ} \mathrm{C}$. The emission and absorption characteristics of nanocrystalline silicon/neodymium:YAG were also investigated. The silicon/neodymium:YAG powder exhibited similar absorption behavior as single-crystal neodymium:YAG. This work has potential use for applications in high-power solid-state lasers.

\section{Citations}

An LN, WX Xu, S Rajagopalan, CM Wang, H Wang, Y Fan, LG Zhang, DP Jiang, J Kapat, L Chow, BH Guo, J Liang, and R Vaidyanathan. 2004. “CarbonNanotube-Reinforced Polymer-derived Ceramic Composites." Advanced Materials 16(22): 2036-2040.

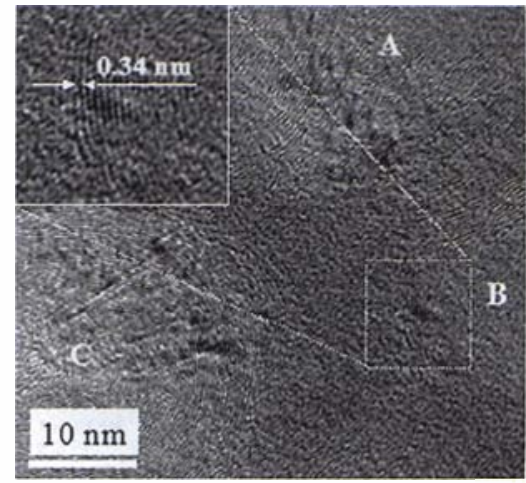

Figure 4-133. HRTEM image of carbon nanotubes within polymer-derived ceramic matrix. 


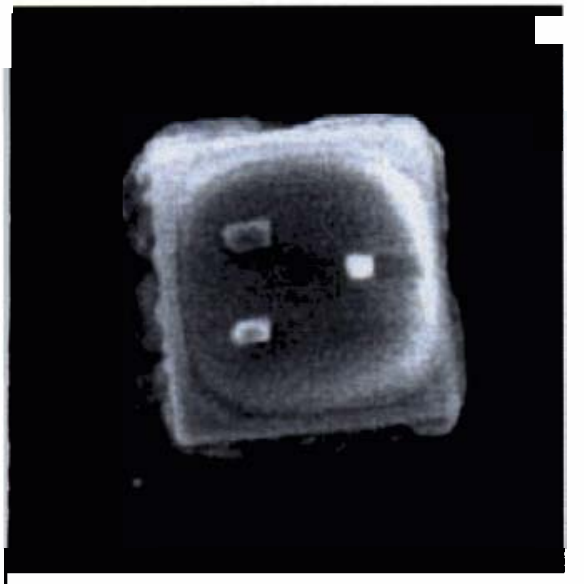

Figure 4-134. An image of $\mathrm{NaOH}$ coating an $\mathrm{NaCl}$ particle obtained using the environmental scanning electron microscope in EMSL's Chemistry and Physics of Complex Systems Facility. This image was recently published in Science (Laskin et al. 2003).
Dhamne A, W Xu, BG Fookes, Y Fan, L Zhang, S Burton, J Hu, J Ford, and L An. 2005. "Polymer-Ceramic Conversion of Liquid Polyaluminasilazanesfor SiAlCN Ceramics." Journal of The American Ceramic Society (submitted).

Wang Y, L Zhang, Y Fan, J Luo, DE McCready, CM Wang, and L An. 2005.

"Synthesis, Characterization, and Optical Properties of Pristine and Doped Yttrium

Aluminum Garnet Nanopowders." Journal of the American Ceramic Society 88(2):284-286.

\section{Reactions at Interfaces as a Source of Sulfate Formation in Sea-Salt Particles}

A Laskin, ${ }^{(a)}$ D Gaspar, ${ }^{(a)}$ W Wang, ${ }^{(b)}$ S Hunt, ${ }^{(b)}$ J Cowin, ${ }^{(c)}$ S Colson, ${ }^{(c)}$ and B Finlayson-Pitts ${ }^{(b)}$

(a) W.R. Wiley Environmental Molecular Sciences Laboratory, Richland, Washington

(b) University of California, Irvine, California

(c) Pacific Northwest National Laboratory, Richland, Washington

Understanding the formation of sulfate particles in the troposphere is critical because of their health effects and direct and indirect effects on radiative forcing, hence on climate. Laboratory studies of the chemical and physical changes in $\mathrm{NaCl}$, the major component of sea-salt particles, show that $\mathrm{NaOH}$ is generated upon reaction of deliquesced $\mathrm{NaCl}$ particles with gas phase $\mathrm{OH}$. 'The increase in alkalinity will lead to an increase in the uptake and oxidation of SO2 to sulfate in sea salt particles.

Atmospheric models typically overestimate the amount of SO2 and underestimate the amount of sulfate near the Earth's surface. 'The explanation for this discrepancy may lie in the chemistry of sea salt particles thrown into the atmosphere by wind and waves. Using particles of $\mathrm{NaCl}$, researchers at PNNL have demonstrated that salt can react with atmospheric hydroxyl radicals (formed from the reaction of ozone, light, and water vapor) to produce $\mathrm{NaOH}$, which is shown coating a salt particle in Figure 4-134.

'The $\mathrm{NaOH}$ increases the $\mathrm{pH}$ of the salt particles, and the alkalinity in turn promotes the oxidation of $\mathrm{SO}_{2}$ by ozone to yield sulfate. In a publication of this work (Laskin et al. 2003), the researchers noted, "'This chemistry is missing from current models, but is consistent with a number of previously unexplained field observations."

Citation

Laskin A, DJ Gaspar, W Wang, S W Hunt, JP Cowin, SD Colson, and BJ Finlayson-Pitts. 2003. "Reactions at Interfaces as a Source of Sulfate Formation in Sea-Salt Particles." Science 301:340-344. 


\section{EMSL Scientific Grand Challenges}

At EMSL, groups of users are focusing on important scientific challenges that require a significant fraction of EMSL resources for considerable periods of time. As a result, Scientific Grand Challenges - coordinated, multi-investigator research focused on resolving a major scientific problem — are being implemented at the user facility. "These Scientific Grand Challenges will:

- Focus on critical milestones in the advancementor use of science

- Support DOE mission areas

- Be user driven

- Take full advantage of EMSL's unique resources and technical expertise

- Increase the scientific impact and robustness of EMSL as a user facility.

The scope of the scientific problems to be addressed will involve multidisciplinary teaming, and the breadth of a challenge will be of such magnitude that it cannot be addressed at any other existing single facility. EMSL's Scientific Grand Challenges will require not only the capabilities of the facility, but possibly other DOE user facilities and specialized instrumentation and capabilities at the participants' home institutions. They will also require significant management and coordination to meet their aggressive goals and are expected to attract and involve users who are among the best scientists in the world.

EMSL has currently launched two Scientific Grand Challenges, one in biogeochemistry and another in membrane biology. Both are funded at approximately $\$ 2 \mathrm{M} /$ year (using a combination of EMSL operating and PNNL internal dollars) for three to five years.

\subsection{1}

\section{Biogeochemistry Scientific Grand Challenge}

\section{Background}

EMSL initiated planning for a BiogeochemistryGrand Challenge in alignment with the DOE Office of Biological and Environmental Research's Environmental Remediation Sciences Division in September 2002. The planning activity, closely coordinated with Environmental Remediation Sciences Division programmatic staff, culminated in November 2003 with a workshop of international experts in microbiology, biology, and geochemistry that identified high-impact topics for the Scientific Grand Challenge. The deliberations of the workshop and the recommended topics were summarized in a workshop report "Earth Life Interaction at the Microbe-Mineral Interface" written by John Zachara and Jim Fredrickson, the PNNL coordinators. "The report has been 


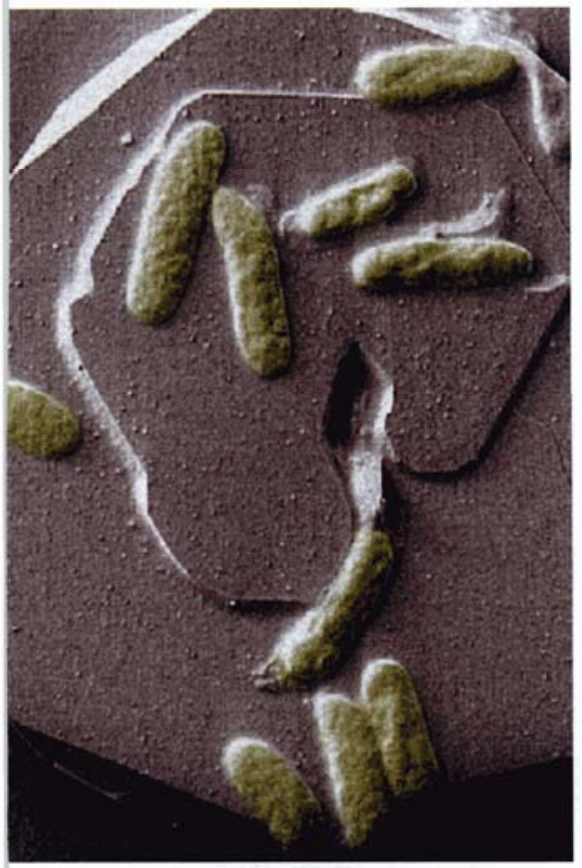

Shewanella oneindensis delivered to DOE, PNNL management, the Biogeochemistry Scientific Grand Challenge advisory panel, and the workshop participants.

\section{Science Question Driving the Scientific Grand Challenge}

'Ihe science question driving this Scientific Grand Challenge is as follows:

Do multiheme cytochromes associated with the outer membrane of metal-reducing bacteria (mtrC in Shewanella oneidensis and omcB in Geobacter sulfurreducens, for example) transfer electrons to $\mathrm{Fe}$ (III) and Mn(IV) oxides and if so, by what mechanisms?

'Ihis topic was selected because it aligned with a high-impact science topic identified at the November 2003 workshop, as well as with EMSL's experimental and computational capabilities and PNNL science expertise. The answer to this question has potential to greatly advance the understanding of bioreductive geochemical processes that regulate contaminant migration in subsurface and sediment/water systems.

\section{Scientific Brief}

Knowledge of the genomes of Shewanella oneidensis and Geobacter sulfurreducens and well-developed genetic systems will greatly facilitate the proposed experimental investigations. 'Ihe cloning and expression system developed for $S$. oneidensis as part of Genomes-to-Life projects will be leveraged to allow relatively rapid production of sufficient quantities of outer membrane protein $\mathrm{mtrC}$ for research. 'Ihe structures of $\mathrm{mtrC}$ from Shewanella and omcB from Geobacter, which are expected to be different, will be determined by NMR spectroscopy and XRD, and the biophysical properties of their heme centers characterized by EPR spectroscopy and other methods. Structural and biophysical information on $\mathrm{mtrC}$ and omcB and their heme groups will be used to develop molecular models to compute their conformation before and after surface engagement, and also to compute electron transfer rates and mechanisms with solid-phase and soluble electron acceptors in different electrostatic and hydration environments as may occur on and within the outer membrane.

In vitro experiments will be performed with $\mathrm{mtrC}$ to understand the fundamental mechanisms of its interfacial engagement with mineral surfaces. $\mathrm{MtrC}$, and other companion proteins as necessary such as mtrB and $\mathrm{mtrA}$, either as free cytochromes or embedded in suitable simulants of the cell envelope (e.g., micelles), will be used with artificial electron donors to probe the formation of electron transfer precursor surface complexes and rates of electron transfer to $\mathrm{Fe}(\mathrm{III})$ and $\mathrm{Mn}(\mathrm{IV})$ sites in oxide surfaces. Epitaxially grown thin oxide films and mineral electrodes will be used to investigate the metalloprotein electrochemistry, surface coordination and site specificity, and the kinetics and thermodynamics of the cytochrome/mineral interfacial electron transfer reactions. 'Ihe influence of mineral surface properties such as structure, defects, and the electronic properties thereof will be investigated and monitored with modern surface science methods. Integrated molecular modeling of the biochemical and mineral surface system components will provide insights on the roles of protein surface conformation and orientation, the reorganization energy, 
and electronic coupling between the cytochrome heme centers and the valence and conduction bands of the oxide.

In vivo experiments will exploit genome information to develop mutants of S. oneidensis and G. sulfurreducens where genes related to outer membrane cytochromes [e.g., their regulation, cellular location, secretion pathway, electron transfer partner(s)] are deleted or over-expressed for hypothesis evaluation.

Mutants and the wild types cultured to specific and reproducible metabolic states will be contacted with model and natural mineral surfaces to evaluate whole-cell electron transfer rates and controlling factors. New high-resolution electron, soft x-ray, and force microscopy techniques will be developed to determine the architecture of the microbe-mineral interface and the molecular environment within the interface where the cytochrome-mineral interaction occurs.

Integration of these studies with the others described above will provide the first comprehensive understanding of how outer membrane cytochromes accomplish biodirected electron transfer with mineral surfaces.

\section{Science Teams and Logistics}

EMSL's Biogeochemistry Scientific Grand Challenge is coordinated by PNNL researchersJohn Zachara and Jim Fredrickson who, along with several other lead scientists, will integrate individual research components to resolve the science question driving this Scientific Grand Challenge. Internal and external science teams will interact and coordinate closely with each other and engage with EMSL staff to resolve specific science needs related to the topic.

The internal science team is drawn from PNNL's Biological and Chemical Sciences Divisions. The following are the team members and associated roles:

- Tjerk Straatsma and Michel Dupuis - Biophysical modeling of cytochromes and hemes

- Michael Kennedy and Michael Bowman - Structural characterization of cytochromes with NMR and EPR

- Margie Rornine and Alex Beliaev - Microbial genomics and mutagenesis

- Kevin Rosso and Eugene Ilton - Mineral physics, surface chemistry, and electron transfer

- Peter Lu - Scanning probe and single molecule spectroscopy

- Scott Chambers - Oxide thin film synthesis and surface science

- Liang Shi - Cloning, expression, and purification of mtrC and related proteins

- Yuri Gorby - Bacterial culturing and physiology

- John Zachara and Jim Fredrickson - Biogeochemistry of microbial metal reduction.

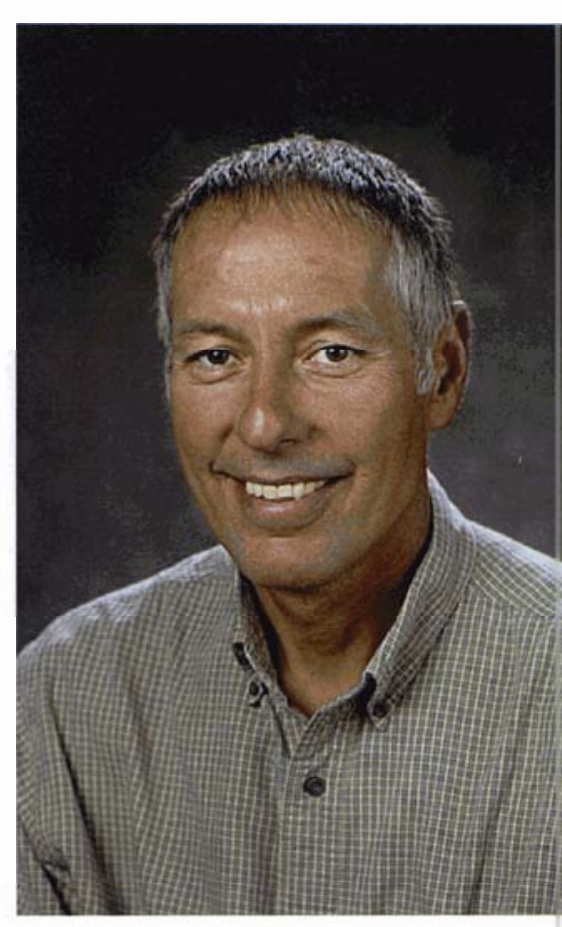

JohnZachara 


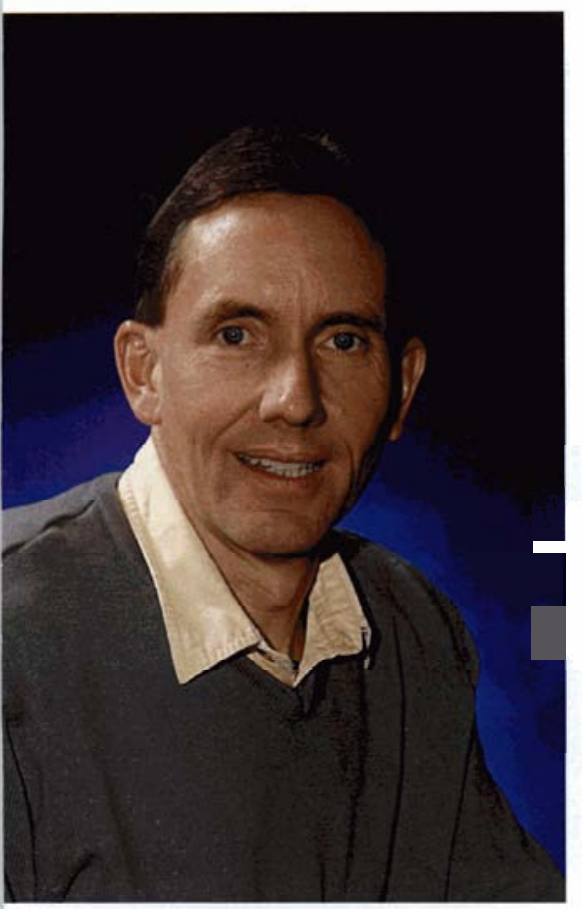

'Ihe external science team is drawn from the university community. 'Iheir names, affiliations, and contributions are as follows:

- Carrick Eggleston, University of Wyoming - Cytochrome interaction with mineral surfaces

- David Richardson, University of East Angleia - Metalloprotein spectroscopy, electron transport in cytochromes, and bacterial electron transport systems

- Terrance Beveridge, University of Guelph - Ultra-structure of the microbemineral interface

- Michael Hochella, Virginia Polytechnic Institute - Mineral electronic properties and biologic force microscopies

- Derek Lovley, University of Massachusetts - Cloning, expression, and purification of omcB and related proteins from Geobacter

- Gill Geesey, Montana State University - Microchemistry and dynamics of the microbe-mineral interface

- Kenneth Nealson, University of Southern California - Soft x-ray microscopy of the microbe-mineral interface, Shewanella physiology

- Thomas DiChristina, Georgia Institute of Technology - Genetics and physiology of metal-reducing bacteria

- Scott Fendorf, Stanford University - Molecular surface chemistry of oxide surfaces and hard x-ray spectroscopy.

JimFredrickson

\section{Membrane Biology Scientific Grand Challenge}

\section{Background}

EMSL initiated the Membrane Biology Scientific Grand Challenge in spring 2004 with ads placed in Science magazine and the American Society for MicrobiologyNews that solicited calls for concept papers. Sixteen papers were received and reviewed by internal and external panels for scientific merit, appropriateness for use of EMSL, and relevance to DOE missions. The winning proposal, lead by Dr. Himadri Pakrasi from Washington University at St Louis, was selected. In December 2004, Pakrasi led a workshop at EMSL that brought together scientists from multiple disciplines and institutions to refine the science questions driving this Scientific Grand Challenge as well as identify EMSL capabilities to be used. 


\section{Science Questions Driving the Scientific Grand Challenge}

The ultimate goal of this Scientific Grand Challenge is to use systems biology approaches to determine the underlying network that governs the forms and functions of cyanobacterial membranes and their components. The key questions that confront the researchers are:

-What is the relationship between plasma membrane and thylakoid membrane, two intracellular membrane systems in cyanobacteria?

- How does the transcriptome of cyanobacteria respond to environmental changes such as light intensity, $\mathrm{CO}_{2}$ level, metal concentrations, and circadian rhythm?

- How does the cyanobacterial membrane proteome change under similar perturbations, and how well does such dynamic pattern relate to that of the transcriptome?

-What are the functional changes in key biological membrane processes (energy transduction, photosynthesis, metal homeostasis and signal transduction) under the same environmental perturbations?

'The cellular components are always in flux, and molecular machines assemble, function, and disassemble as a function of time and environmental alterations. It is imperative to use a systems biology approach and integrate all temporal information into a predictive, dynamic model to understand the functioning of a cell, in general, and the cellular membrane systems, in particular.

\section{Scientific Brief}
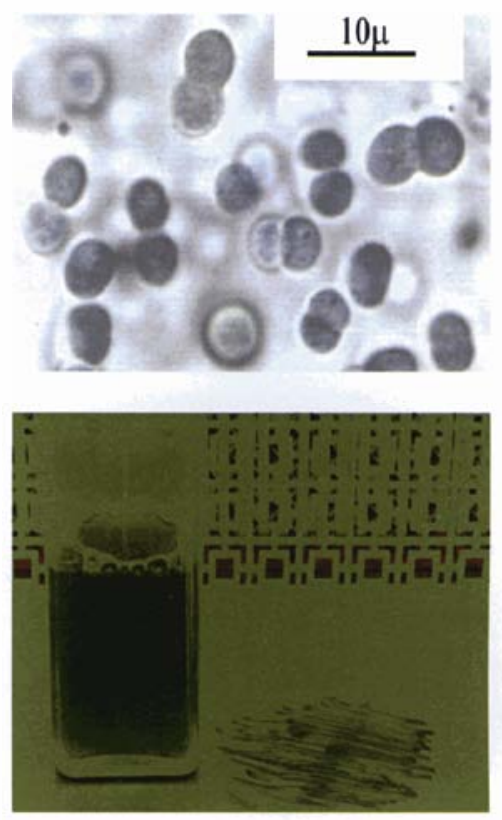

Synechocystis 6803.

The recent achievements in genome sciences have opened new paradigms in biological research. Complete genome sequences have become available for many microorganisms and a few eukaryotes, including humans and plants. To extract useful information about the biological functions of any organism from such sequences, the challenge is to develop and use new computational and experimental tools to integrate data at various levels of biologically relevant interactions. Such a "systems biology" approach should provide enabling technologies to examine complex biological processes, which should in turn result in an integrated and predictive understanding of how an organism behaves and responds to environmental changes.

Clearly, the successful implementation of such a systems approach will require collaborative interactions among biologists, computer scientists, mathematicians and model builders, engineers, physicists, chemists, and perhaps specialists in other disciplines. 'The current call for a Scientific Grand Challenge in membrane biology at PNNL offers an excellent and timely opportunity to assemble a team of academic researchers, and scientists at PNNL to initiate a systems biology project for a fundamental understanding of membrane processes in cyanobacteria. 


\section{Organisms}

Cyanobacteria are oxygenic photosynthetic prokaryotes that make significant contributions in harvesting solar energy in the biosphere, planetary carbon sequestration, metal acquisition and Hz-production. The genomes of more than a dozen different cyanobacterial species have been sequenced. During this project, team members plan to focus on two cyanobacterial species: Synechocystis sp. PCC 6803, and Cyanothecesp. ATCC 51142. Synechocystis6803 is a fresh

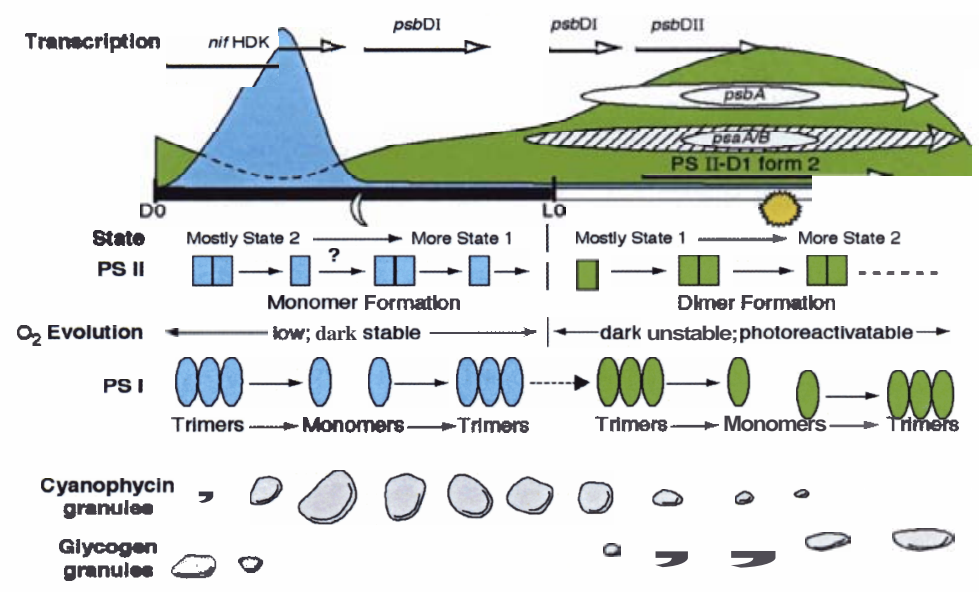
water organism, and is arguably the most widely studied cyanobacterium during the past decade. It is also the first photosynthetic organism with a completely sequenced genome (1996), and has nearly 3700 annotated genes. This species is naturally transformable, and targeted gene replacements can be readily generated. Cyanothece is a marine organism that is capable of oxygenic photosynthesis, nitrogen fixation as well as heterotrophic growth in the dark. Most importantly, this organism displays one of the most striking circadian rhythms identified in anyprokaryote. This is needed because the enzyme that fixes atmospheric N2, nitrogenase, is highly sensitive to oxygen, an obligatory product of oxygenic photosynthesis. Because this is a

Figure 4-135. Diagram of metabolic rhythms in Cyanothece 51142. This summary indicates the diurnal patterns of $\mathrm{N}_{2}-$ fixation in the dark and photosynthesis in the light. unicellular organism, it cannot form specialized cells to protect its nitrogenase. 'Thus, the strain uses time as a way of separating the peaks of photosynthesis from peaks of nitrogenase activity (Figure 4-135). 'The genome of Cyanothece 51142 has not been sequenced yet. Recently, Pakrasi and Sherman have submitted a proposal to the Washington University Genome Sequencing Center for draft sequencing of this genome.

\section{Controlledcultivation}

For this Scientific Grand Challenge, it is imperative that the cyanobacterial cultures are grown in continuous or semi-continuous mode in illuminated fermentors (photobioreactors). Cultivation technologies must be used that provide continuous monitoring and control of culture conditions so that wellcharacterized and reproducible samples are produced. The microbial growth facility at PNNL will soon have fiber optics illuminated fermentors for the cultivation of photosynthetic organisms in place. Both Synechocystis 6803 and Cyanothece 51142 strains will be grown in such photobioreactors under defined environmental conditions (light, $\mathrm{CO}_{2}$, metal concentrations etc.). Most importantly, cultures will be grown in sufficiently large volumes so that aliquots of the same sample are used for parallel transcriptomics, proteomics, ultrastructural, and functional studies. Careful analysis of such well-defined data sets will greatly facilitate the network inference and model-building activities.

\section{Imaging of cyanobacterialcells}

Like other bacterial cells, cyanobacteria are relatively small in size. Advanced electron microscopy techniques will be used to obtain high-resolution, albeit 
static pictures of the membrane architecture in both Synechocystis 6803 and Cyanothece 51142 cells. In addition, correlated atomic force microscopy and confocal fluorescence lifetime imaging microscopy expertise at EMSL will be accessed to examine the dynamic behaviors of the membrane systems in live cyanobacterial cells.

\section{Microarray analysis of the cyanobacterialtranscriptome}

For global gene expression studies, two different versions of DNA microarrays for Synechocystis 6803 are available: a spotted array that has PCR fragments corresponding to the majority of the chromosomal genes on it and a more recently printed 60-mer oligonucleotide microarray that has been produced by the Agilent Co., and includes an additional 460 plasmid-encoded open reading frames.

The microarray measurements will be differential expression values of the perturbed state relative to the unperturbed state. Different perturbations will be used, such as light intensity, $\mathrm{CO}_{2}$ concentration, and available metal (iron, manganese, zinc, and copper) concentrations. For Cyanothece, samples will be collected at various stages of the circadian cycle.

\section{Analysis of the Dynamic Nature of Cyanobacterial Membrane Proteomes}

A specific objective here is to enable the studies of comprehensive and quantitative proteomics (both global and subproteomic), and the kinetics associated with specific protein complexes from Synechocystis $\mathbf{6 8 0 3}$, that has been cultured under experimental conditions designed to follow the incorporation of ${ }^{15} \mathrm{~N}$ as a stable isotope pulse label. Operationally, the cell can be subdivided into as many as seven fractions: outer membrane, periplasm, plasma membrane, cytoplasm, carboxysome, thylakoid membrane, and thylakoid lumen. Determining the relative abundance of proteins in Synechocystis cells exposed to different environmental conditions is essential to the study of cellular metabolism and responses to environmental changes. In addition to the characterization of the global proteome of Synechocystis through the development of the AMT tag database, the characterization of six membrane-associated as well as the cytoplasmic subproteomes derived from subcellular fractionation will be accomplished. Of significantinterest is information on both relative protein abundances and on the absolute abundances of proteins and their modifications. The advanced capabilities in membrane proteomics at EMSL should help fill this significant gap in the basic knowledge about cyanobacterial membranes.

\section{Functionalassays}

$\boldsymbol{A}$ variety of assays will be used to determine the functional properties of the cyanobacterial cells under different environmental conditions. For example, room temperature fluorescence kinetics analysis will be employed for noninvasive measurements of photosynthetic electron transfer processes. Inductively coupled plasma mass spectrometry will be used to profile the metal contents of various cellular and subcellular preparations. In Cyanothece, the dynamic nature of cellular physiology will be monitored by measuring $\mathrm{N}_{2}$ fixation (a dark function) and photosynthetic $\mathrm{O}_{2}$ evolution (a light function). Biochemical assays for the accumulation of glycogen (in the light) and accumulation of cyanophycin 
(an amino acid polymer that accumulates in the dark after $\mathrm{N}_{2}$ fixation) will determine the major endpoints of central metabolism. Most importantly, collaborations will be initiated with specialists in the EMSL's High-Field Magnetic Resonance Facility to develop and use EPR and NMR-based assays to track critical electron and proton transfer reactions during cyanobacterial energy transduction processes.

\section{Network inference and model building}

Effective progress in this Scientific Grand Challenge will clearly require interactions among biologists, computational scientists, and other scientists and engineers. These interactions are necessary to develop not only the means to acquire the large, complex data sets required to understand the properties of complex biological systems, but also the tools to rapidly analyze those data. The challenge is to provide methodologies for transforming high-throughput data sets into biological insights about the underlying mechanisms. An essential feature of systems biology is the construction of predictive models. The Ghosh and Aurora groups plan to collaborate with Steve Wiley, George Michaels, and colleagues in PNNL's Biomolecular Systems Initiative for the network generation and model-building activities.

\section{Managementplan}

As the project leader, Himadri Pakrasi will be responsible for overall coordination, whereas each co-investigator will coordinate the activities in his own research group. Pakrasi will also interact with the coordinator of the PNNL science contributor team to facilitate overall research activities in this project.

\section{Science Team}

The following are members of the Membrane Biology Scientific Grand Challenge:

- Himadri B. Pakrasi, project lead - Department of Biology, Washington University

- Louis A. Sherman - Department of Biological Sciences, Purdue University

- Teruo Ogawa - Department of Biology, University of Tokyo

- Bijoy K. Ghosh - Department of Electrical and Systems Engineering, Washington University

- Rajeev Aurora - Department of Electrical and Systems Engineering, Washington University.

Key PNNL collaborators and their roles:

- Richard D. Smith and David G. Camp - High-throughput membrane proteomics

- Yuri A. Gorby - Controlled cultivation of cyanobacteria

- Alice Dohnakova, Gala Orr, and Peter Lu - Image analysis of cyanobacterial cells 
- Chris Oehman and H. Steven Wiley - Network inference and modeling

- Michael Kennedy and Michael Bowman - Structural characterization with NMR and EPR.

\section{4}

\section{Collaborative Access Teams}

CATs allow researchers to conduct high-impact science that demonstrates the capabilities and value of EMSL and helps EMSL to remain at the forefront of science. 'These teams provide a mechanism to attract and increase the number of high-impact users in a focused research environment and build new capabilities for use by the CAT and general EMSL users.

CATs are comprised of nationwide teams of scientists who use EMSL facilities and capabilities to conduct funded programmatic research around focused science themes. Membership in a CAT is open to all members of the scientific community who can contribute significantly to the CAT.

EMSL is currently in the process of rolling out several CATs. A staged approach is being taken to ensure that the CATs are focused around common themes.

\subsection{1}

\section{Structural Genomics CAT}

'The Structural Genomics CAT is led by PNNL researcher Michael Kennedy and is comprised of more than 35 scientists from 16 different institutions. 'This CAT will carry out research focused on structural genomics and related research areas with the primary goal of increasing throughput, maximizing efficiency of existing resources, and developing technology to increase throughput and efficiency.

A significant amount of CAT resources will support the research objectives of the NESG, a consortium of 10 institutions involving 23 NMR scientists. 'The objective of the NESG is to produce a high volume of protein structure data and to test the feasibility of high-throughput, NMR-based structural genomics, as per the guidelines of the National Institutes of Health Protein Structure Initiative. Structural genomics defines an ultimate goal of determining the three-dimensional structure of all proteins encoded by an organism's genome. Each three-dimensional structure can either be derived experimentally, or given a sufficient sequence similarity, can be modeled by homology from an appropriate experimentally derived structure. An important objective of the National Institutes of Health Protein Structure Initiative pilot centers is to test feasibility of various approaches for high-throughput structural genomics. A critical concept being assessed is what role that NMR spectroscopy should play in structural genomics. 


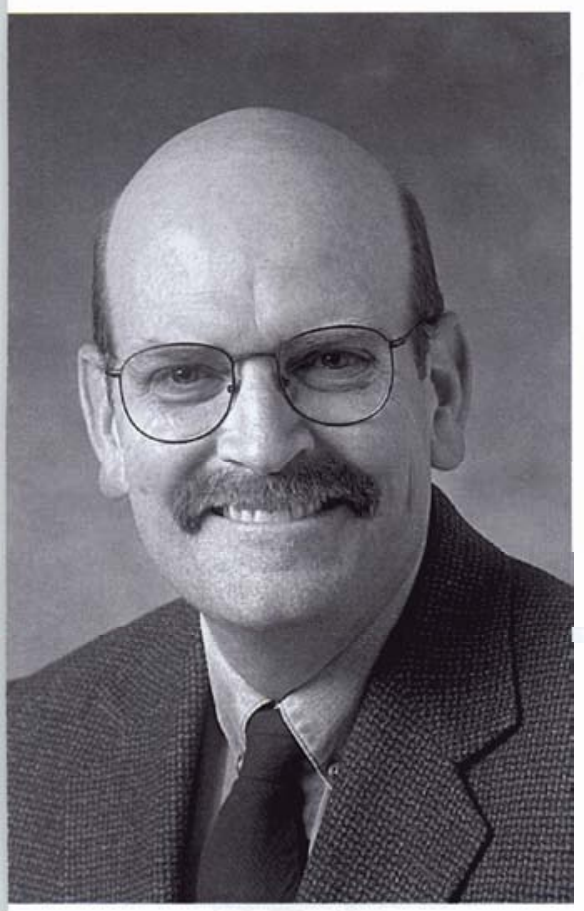

Other projects involved in the Structural Genomics CAT include a Lawrence Berkeley National Laboratory structural biology project that is focused on solving the structure of whole classes of DNA repair proteins; a Battelle/Chiron project that investigates essential genes in S. aureus; and a Battelle-supported project involving Massachusetts General Hospital's Harvard Medical School that is studying the structure of large groups of proteins encoded by essential genes in Pseudomonas aeruginosa.

'This CAT will partition its time among several activities, with the major objective being high-throughput protein structure determination. In support of this objective, a considerable fraction of resources will be used for data collection for NESG and the other National Institutes of Health-supported Protein Structure Initiative pilot centers. Another objective is to develop new pulse sequences and approaches for reducing the overall resources required for each protein structure determination. Finally, this CAT will develop automation technologies for data collection and data management.

\subsection{2}

\section{Catalysis CAT}

Catalysis continues to be vital to the chemical and petroleum industries, and to the development of pollution abatement technologies. Catalysis and catalytic processes account for nearly 20 percent of U.S. gross domestic products and nearly 20 percent of all industrial products; the economic impact of catalysis is estimated to be more than $\$ 10$ trillion per year worldwide. As new products become ever more sophisticated, the need to quickly develop new catalysts grows rapidly in importance. A fundamental understanding of chemical transformations is needed to enable scientists to address the grand challenge of the precise control of molecular processes by using catalysts. In particular, ideally catalyst design should be able to directly arise from a molecular-level understanding of exactly how catalysts function, thereby providing ways to "tune" the catalyst for optimum activity with minimal production of undesirable side products. Due to recent and rapid advances in experimental catalysis science methodology, computational methods, and computing power, a real opportunity exists to strongly couple theory and experiment in order to provide profound insights into catalyst behavior, enabling the design of new catalysts.

Investigators in EMSL's Catalysis CAT will use this approach, taking advantage of unique experimental and computational resources at EMSL to conduct basic and applied research in the area of heterogeneous catalysis. 'The overall effort will be coordinated by PNNL researcher Chuck Peden. A specific focus of the Catalysis CAT will be to develop a fundamental understanding of transitionmetal oxide-catalyzed chemical transformations so that new catalysts can be systematically developed from first principles. The proposed research efforts will endeavor to critically relate the surface chemistry, surface morphology, atom connectivity, and pore dimensions for controlling catalytic activity and selectivity of target acid-base and redox reactions via: 
- molecular-level simulations of heterogeneous catalysts

- synthesis and characterization of heterogeneous catalysts using methods that provide nanoscale precision in the type, number, and distribution of active sites

- performance and detailed kinetics measurements on model and realistic catalytic systems

- development and testing of new experimental approaches for addressing complex catalysis issues.

In addition, the Catalysis CAT will enhance the overall scientific impact of the individual efforts of CAT members. A goal of this CAT is to provide basic science experimental and computational resources to the CAT members. For example, a significant quantity of EMSL high-performance computer time is available to the Catalysis CAT through the current Molecular Science Computing Facility Computational Grand Challenge on Catalysis.

In order to achieve these objectives, a team of investigators is assembled, including PNNL staff and a broad range of external users with a wide range of expertise in catalysis. The efforts of the individual users in this CAT are supported by a variety of sources, including DOE's Office of Basic Energy Sciences/Division of Chemical Sciences; DOE's Office of Energy Efficiency and Renewable Energy; DOE's Office of Fossil Energy; DOE's Mathematics Information and Computer Sciences program; industry direct-funded projects; and the National Science Foundation. EMSL operations funding is also expected to be available for user support and consulting and next-generation instrumentation and software development.

\subsection{3}

\section{Analytical Mass Spectrometry CAT}

DOE has a long-established key role in advancing the technique of mass spectrometry as an investigative tool for physical chemistry and characterization of complex mixtures of materials. Beginning with its origins in the Manhattan Project of World War II and continuing to the present time, DOE researchers have been responsible for key innovations in instrumentation and techniques based on mass analysis. This tradition is continued in this CAT, which has distinguished itself in development of novel instrumentation and deploying it for determining key thermodynamic and kinetic parameters for complex ions. A special focus is energizing and dissociating ions by impacting them on semiconducting films and following the kinetics of dissociation as a function of time following ion impact. The special characteristics of this means of activating ions-in particular, precise control of energy exchange, the ability to transfer large amounts of energy in a very short time, and robustness of these films-make this technique very attractive for chemical analysis.

Led by EMSL researcherJulia Laskin, the general objective of this CAT is to achieve a fundamental understanding of the reaction kinetics and dynamics of 
activating and dissociating complex molecular ions. 'These processes are the scientific foundation for MS/MS, one of the most important analytical methods developed in the last century. "The collisional activation and dissociation of protonated model peptides are being investigated, a particularly important class of complex molecules whose characterization by MS/MS is central to the emerging field of proteomics. Results obtained thus far indicate that collisional activation of ions in a single collision with a specially prepared surface-surfaceinduced dissociation-is a highly promising method for investigating fundamental questions of ion activation and may be a preferred method for practical applications, especially in FTICR mass spectrometry. Dynamics of the surfaceinduced dissociation of large molecules is being investigated, the influence of physical and chemical properties of surface-induced dissocation targets on energy transfer efficiency and neutralization of complex ions using collision energyresolved and angular-resolved measurements. 'Theoretical models will also be applied to extract the energetics and dynamics of complex ion dissociation processes from experimental data.

\subsection{4}

\section{Atmospheric Emissions CAT}

Director's note: Due to a change in CAT leadership, EMSL is currently working to redefine this $C A T$ and identify leadership. As a result, this $C A T$ is not currently active.

Understanding energy-related pollutants is an urgent need for DOE, as existing and proposed regulations of those emissions will strongly impact energy use. Much of the uncertainties concern the aerosols produced, as they influence climate, visibility, and human health. The purpose of this CAT is to create a unique research capability with an international user base to understand the complex gas-aerosol and aerosol-cellular chemistries and its policy implications. No such center exists, within or external to the national laboratory system. EMSL's uniquely extensive aerosol and trace gas characterization capabilities; instrument development mission; advanced computing resources; and the strength of PNNL in computer modeling of weather, climate, and pollution; strong-field measurement capability of the atmosphere; and cellular-level understanding of lungs form the core of this initiative. 'This CAT will also strongly benefit from the uniquely comprehensive PNNL environment that includes policy, inhalation toxicology, vehicle emissions control, homeland security, and industry connections.

Atmospheric aerosols are complex chemical systems involving interactions among gases, liquids, and solid particulate matter that range from the nanometer to microcurie size and are composed of both inorganic and organic constituents.

'The biggest uncertainty in understanding global industrialization-driven atmospheric climate change is due to the poor understanding of the absorption and scattering of sunlight by atmospheric aerosols, their role in seeding clouds and haze, and their lifetime. Emission of aerosols from vehicles and industries is 
regulated by environmental law to protect human health, but currently with little insight as to what particles are dangerous and why. Complying with regulations on regional air pollution to limit haze and inhaled particles requires a better knowledge of aerosol chemistry and sources. Atmospheric aerosols also play important but poorly parameterized roles in trace gas chemistry like $\mathrm{NO}_{2}$ and $\mathrm{SO} 2$ oxidation that control haze and ozone production. These needs drive the requirement for aerosol research.

Laboratory studies will seek to understand how aerosol particles change as they age and react under well-controlled conditions, with the goal to understand how the changing composition leads to changes in their impact on regional and global environment and to health effects. Measuring aerosols in the "real world" is a crucial part of this CAT. PNNL's G1 Gulfstream research aircraft is one of only a few large research aircraft suited for work in atmospheric aerosol sciences. This CAT will develop and deploy enhanced mobile sampling capabilities from trucks, from the air (the G-1 aircraft) and on the individual (personal exposure monitoring). These laboratory and field measurements can be synthesized into quantitative models through use of the EMSL's world-class supercomputer to allow better air quality and climate change predictions. Health effect mechanisms will be explored as well, not only via the aerosol characterization in the laboratory and field, but also by unique PNNL/EMSL capabilities in genomics and proteomics, toxicology expertise, and collaboration with outside researchers.

New funding opportunities are expected from the DOE Transportation Technology, DOE Fossil Fuels, the Environmental Protection Agency, the National Institutes of Health, and the Department of Defense and Homeland Security, and possibly DOE's Assessment and School Performance and Atmospheric Radiation Measurement programs. University partners will also solicit National Science Foundation funding, with funding via industrial consortiums or state air quality boards possible. The large-field missions should attract many outside collaborators, who will be independently funded. 
5

\section{EMSL User Outreach and Administration}

\section{1 \\ EMSL Outreach}

'Ihe goal of EMSL's outreach strategy is to enable its vision to be recognized as a science-to-solutions leader who provides the catalyst for assembling teams of expert scientists to address challenging research objectives that demand multidisciplinary approaches. To achieve this vision and establish an engaged, proactive user community, EMSL has designed outreach strategies to:

- Attract high-profile users

- Build visibility within the science community

- Foster collaborations with PNNL researchers

- Promote educational outreach.

\subsection{1}

\section{Attract High-Profile Users}

Attracting high-profile scientists as users will enhance EMSL's scientific reputation and increase its visibility and appeal to the scientific community. 'Through their research activities and collaborations at EMSL, these high-profile users will help further develop the distinctive signature scientific areas for which EMSL is known. Additionally, these users bring to EMSL mentoring and scientific collaboration opportunities for early-career staff and users. 'Ihe following activities must be undertaken to meet this goal:

- Confer with sponsors and EMSL's User Advisory and Science Advisory committees to define "high profile" (e.g., Distinguished Visitors are defined as individuals who are Endowed Chairs, Essential Science Indicators, National Academy Members, and/or Nobel Laureates).

- Identify high-profile scientists whose expertise falls in the areas of strategic focus. 'Ihis would require networking with EMSL management and EMSL/PNNL staff to identify current research activities, existing external relationships, and any potential high-profiles users - while also soliciting the input of Battelle Fellows and the User Advisory and Science Advisory committees.

- Develop and implement activities and opportunities that would attract highprofile users; for example, leveraging connections of the PNNL research directorates, offering Wiley Distinguished Fellowships, and sponsoring visits and seminars at EMSL. 
These proposed activities would also leverage EMSL's goal to foster collaborations with PNNL researchers; here, senior PNNL researchers would be encouraged to promote collaborations with these high-profile users while making use of EMSL capabilities and resources.

\subsection{2}

\section{Build Visibility within the Science Community}

EMSL is well known among select groups in scientific and/or regional domains, but the user facility is not widely known among the scientific communities that represent EMSL's main science thrust areas. Communication and demonstration of EMSL's scientific capabilities and research accomplishments to the scientific community would result in the recognition of EMSL as a unique user facility - which in turn would result in advocacy of EMSL's capabilities at external institutions and to EMSL sponsors and stakeholders. The following activities must be undertaken to meet this goal:

- Identify leading institutions and external researchersin EMSL distinctive signature areas. Also identify national/regional meetings on topics of relevance to EMSL distinctive signatures.

- Develop and implement activities and opportunities to build a reputation among the science community. This would include ensuring that EMSL is acknowledged in journal articles and presentations; holding onsite workshops on scientific areas of focus; promoting direct communications between research staff and external scientists at identified institutions; and providing support to EMSL staff who are organizing meetings and sessions at technical conferences.

- Confer with PNNL's Partnership groups to determine if joint objectives would be met by cost sharing to bring academic scientists to EMSL for invited seminars.

EMSL's approach to meeting this goal must be fostered by users, staff, and management alike; for example, staff and users must consistently acknowledge EMSL's role in developing solutions to complex scientific challenges. Likewise, organization of onsite workshops in EMSL distinctive signatures and sponsorship of symposia at national/regional meetings will serve to increase and strengthen the reputation and recognition of EMSL. 


\section{1 .3}

\section{Foster Collaborations with PNNL Researchers}

PNNL is rich with scientific expertise and technology development abilities. 'Thus, the development of leading-edge capabilities at EMSL requires collaboration with PNNL scientific leaders. Promoting collaborations among EMSL and PNNL researchers will enable new capability development through joint grant proposals and more efficient use of PNNL-wide resources and intellectual pool - resulting in strengthened EMSL and PNNL programmatic activities. To facilitate these collaborations, the following actions are planned:

- Identify key existing strategic PNNL relationships, as well as potential key PNNL relationships in EMSL's scientific areas of focus.

- Develop and implement activities and opportunities to foster collaborations with PNNL researchers, such as engaging stakeholders by routinely inviting relationship managers and division directors to EMSL for meetings and seminars, and establishing "brown bags ${ }^{\mathrm{n}}$ and networking opportunities.

Such activities will help to increase the number of joint EMSL/PNNL grant proposals and the number of those proposals with EMSL staff as principal investigator or co-principal investigator. Concurrently, EMSL capabilities will be enhanced by these collaborations, promoting state-of-the-art research activities.

\subsection{4}

\section{Promote Educational Outreach}

A critical element of EMSL's mission is to educate and train the next generation of researchers. EMSL, as well as PNNL, is committed to supporting science and engineering education. To maximize its impact, EMSL will leverage its activities with those of PNNL's Partnership groups. 'This collaboration will maximize the use of EMSL/PNNL resources and focus on areas of mutual interest, such as outreach to regional universities and enhancement of PNNL visibility to academic scientists. Educational outreach activities that will support this goal are as follows:

Work with PNNL's Partnership groups to identify educational institutions with students and faculty who are compatible with EMSL's scientific thrust areas. This will include inviting these groups to visit and tour EMSL, and holding scientific seminars and workshops at the groups' home institutions.

- Develop and implement activities and opportunities to foster collaborations with targeted educational groups. 'This will include participating at national/ regional meetings as an exhibitor, holding onsite workshops targeted to students and faculty, and sponsoring research collaborations and institutes. 
Such opportunities will also help EMSL achieve its goal of building an effective reputation in the science community and create future collaboration opportunities.

PNNL Partnership groups include University Relations and Fellowship Programs, Science Education Programs, Economic Development Office, and Northwest Regional Programs, with corresponding networks to regional and national entities that could benefit from access to EMSL and provide vehicles for training and/or collaborative use.

\section{2}

\section{EMSL User Administration}

\section{2 .1}

\section{Access to EMSL}

EMSL facilities and capabilities are available to the general scientific and engineering communities to conduct research in the environmental molecular sciences and other EMSL distinctive signature areas.

EMSL supports both open and proprietary research. Open research is basic and applied research in science and engineering where the resulting information is ordinarily published and shared broadly within the scientific community. Users engaged in open research generally are not charged for using EMSL facilities or equipment. Intellectual property developed in the course of open research is protected in accordance with DOE policies, which, in general, assign the intellectual property and any resulting commercial benefits to the discoverer. A limited amount of proprietaryresearch may also be conducted in EMSL under a proprietary sales contract. Under the terms of the DOE class waiver, users engaged in proprietary research are obligated to pay the full-cost recovery rate for use of the facility. In return, the user has the option to take title to any inventions made during the proprietary research program and to treat as proprietary all technical data generated during research.

EMSL strives to provide seamless mechanisms for users to access EMSL capabilities to solve problems that range from small, single-principal investigator projects to large, complex, multi-institution scientific challenges. Users may access EMSL facilities via unsolicited proposals, calls for proposals, CATs, and Scientific Grand Challenges. Regardless of access type, all users and their institutions must sign an EMSL User Agreement and agree to abide by the terms of the User Agreement prior to use of EMSL facilities or capabilities. All users must also be cognizant of, and abide by, EMSL Standard Practices and Procedures.

General support and appropriate training on instruments or other resources will be provided to users by EMSL staff. Scheduling use of the facilities and 
capabilities of the EMSL for approved proposals will be determined by EMSL staff in consultation with users.

Potential users may at any time submit a proposal through the EMSL website (http://www.emsl.pnl.gov). Following submission of the electronic proposal, the proposal author receives a prompt acknowledgment of receipt and, in most cases, within four weeks is advised whether the proposal has been accepted. If the proposed use is approved, the EMSL host contacts the user to coordinate use arrangements. Users are asked to advise the host of any materials, chemicals, or equipment to be shipped or hand carried to EMSL. Questions about procedures, proposal review comments, or appeals may be addressed in writing to the User Services Office (userservices@emsl.pnl.gov).

\section{2 .2}

\section{Peer Review Process and Calls for Proposals}

To select the best and most appropriate science, all EMSL proposals are reviewed using specific criteria, which include scientific merit with respect to EMSL and DOE missions, technical approach, potential environment, safety and health hazards, proprietary status, appropriateness of the requested instrumentation, the contribution that EMSL can have in bringing that science to fruition, and availability of resources and personnel.

EMSL's High-Field Magnetic Resonance Facility and Molecular Science Computing Facility submit regular calls for proposals for use of capabilities such as the high-field NMRs and the 11.8-teraflop supercomputer. Due to the very high demand for these capabilities, proposals undergo a review by external experts.

The four remaining research facilities at EMSL have not historically solicited proposals via targeted calls but welcome and internally review proposals on an as-received basis.

The following describes the calls for proposals and review processes of the HighField Magnetic Resonance Facility and Molecular Science Computing Facility and outline roadmaps for the remaining four research facilities to establish analogous procedures. In addition, plans for developing a web-based proposal review tool are outlined.

\section{High-Field Magnetic Resonance Facility}

The High-Field Magnetic Resonance Facility submits two proposal calls per year (http://www.emsl.pnl.gov/hfmrf/). Proposals are first reviewed by the custodians of the requested instrument for overall appropriateness. Those proposals requesting use of oversubscribed higher-field NMRs are sent for expert review, where two to five external reviewers and two to four internal reviewers examine the proposals (approximately 30 proposals are reviewed during a sixmonth period). Each proposal is then categorized (top 10 percent, 75 to 90 percent, 50 to 75 percent, 25 to 50 percent). Based on a final ranking, proposals 
are approved, with all or part of time requested allocated or denied. All but two weeks (reserved for maintenance) of a 26-week period are allocated for use.

\section{Molecular Science Computing Facility}

'The Molecular Science Computing Facility issues a Call for Computational Grand Challenge Proposals once a year (http://mscf.emsl.pnl.pov/). 'These proposals undergo a rigorous external peer review. The recent call for proposals engaged 22 external scientific reviewers from leading universities and research institutions from around the world. Proposals received two reviews on average, and three reviews in certain cases. In addition to its Computational Grand Challenges, the facility also engages users in Pilot Projects.

- Computational Grand Challenge Projects examine complex problems in science and engineering with broad scientific and environmental or economic impacts whose solutions can be advanced by applying high-performance computing techniques and resources. Only a few large proposals with multiteam, multi-institution participants are accepted each year. Each Computational Grand Challenge project operates for a period of three years.

- Pilot Projects are proposals of general research and are typically directed at developing the capability to submit a Computational Grand Challenge proposal in the future (e.g., a combination of theory/method or code development activities or calculations that provide the initial scientific basis of a Computational Grand Challenge proposal).

Eighty percent of the available resources of the 11.8-teraflop supercomputer is allocated via the external peer-review process to the Computational Grand Challenges. 'The remaining 20 percent is assigned to Pilot Projects (5 to 15 percent), the Office of Science (5 percent), and Scientific Grand Challenge projects. Pilot Projects may be submitted year-round and are reviewed internally.

\section{Roadmaps}

Due to the demonstrated success seen in the high quality of proposals submitted as the result of the calls for proposals by the High-Field Magnetic Resonance Facility and Molecular Science Computing Facility, the four remaining research facilities (Interfacial and Nanoscale Science Facility, Environmental Spectroscopy and Biogeochemistry Facility, Chemistry and Physics of Complex Systems Facility, and High-Performance Mass Spectrometry Facility) are developing roadmaps unique to their instrumentation and research. The establishment of review procedures in all facilities will help ensure that EMSL attracts and accepts the most valuable research, and their development will be implemented through a number of activities, many of which are in process at this time. Roadmaps will contain:

-A list of all available resources, noting oversubscribed instruments

- The amount of resource time available to external, PNNL, and resident users

- Internal peer-review processes 
- External peer-review processes for oversubscribed resources.

The roadmaps will also address:

- Cross-facility proposals and coordination of cross-facility calls for proposals

- Reserving time for Scientific Grand Challenge projects.

Questions to be addressed to ensure that proposed research is well suited to EMSL can include the following:

- Does the research encompass cutting-edge, unique science?

- Are EMSL capabilities essential to perform this research?

- Is research consistent with EMSL user policies and science goals?

- Is research supported by a funded externally peer-reviewed project?

- Is research complimentary to instrument capabilities and staff expertise?

- Does research fall within the EMSL environment, safety, and health envelope?

- Are modifications needed to EMSL equipment or software?

-What are the instrument(s) time requirements?

These roadmaps will be finalized by the end of Fiscal Year 2005 and executed beginning in Fiscal Year 2006. Since each facility is unique and offers different resources and capabilities to the scientific community, the EMSL Director has assigned a point of contact to work with each facility on the creation of these roadmaps. To assist in the implementation of the facility's review processes, the point of contact will help define the requirements for a web-based proposal review tool described below.

\section{2 .3}

\section{Tools to Manage User Proposals and Resource Allocations}

EMSL staff have developed tools to automate and improve the ability to manage user proposals and track use of EMSL resources. The EMSL User System, developed to manage all user proposals, and the EMSL Resource System, designed to schedule and record use of resources, have been operational since 2002. 'These tools have enabled EMSL to effectively manage proposal life-cycles and track use while minimizing the administrative burden of these tasks. In addition, EMSL is launching the development of an electronic proposal review management system in Fiscal Year 2005 to further optimize speed and ease of processing proposals. 


\section{EMSL User System}

'The EMSL User System, an EMSL-developed, online workflow tracking software package, provides users with a means to access EMSL capabilities and provides management with an effective way to track users, projects, and results. To access EMSL, a potential user submits an electronic proposal request via the EMSL User System that lists details about the proposed research. Once submitted, the proposal is immediately routed to the appropriate reviewers. 'Throughout the remainder of the proposal lifecycle, the user and associated EMSL staff receive automated messages indicating proposal status and requesting additional

\begin{tabular}{|c|c|c|}
\hline Proposal & Usage & Extended Usage \\
\hline $\begin{array}{c}\text { Days } \\
\text { (weeks for proposal calss) }\end{array}$ & Up to 1 year & Up to 3 years \\
\hline
\end{tabular}
information or summaries of research. As a centralized repository of data for all EMSL user proposals, the EMSL User System enables easy generation of reports on user statistics, optimizing resources

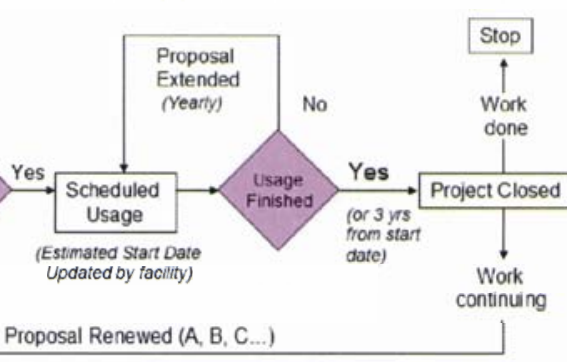
that were previously devoted to compiling data from numerous sources. By automating and centralizing this workflow, user proposals are more efficiently processed, speeding up researcher access to the one-of-a-kind resources and capabilities at EMSL.

Fully deployed in Fiscal Year 2003, EMSL will continue to improve the EMSL User System based on user and staff feedback. 'The software has been demonstrated to other user facilities (i.e., several of the new nanocenters funded by the DOE Office of Basic Energy Sciences) and will be distributed to these facilities upon request.

In the proposal system, the potential user is asked to provide information about the participants, details about the proposed work, and logistics:

- The Participants page requests information about each participant and their related institutions.

- 'The Details page requests the proposal title, abstract and attachments outlining the research, proposed start and end dates, and EMSL resources desired along with their estimated hours of use.

- The Logistics page requests information about the funding agencies, and the samples, chemicals, and user equipment that will be brought to the facility.

The proposal process begins when a proposal is received by EMSL. Once approved, staff associated with the corresponding facility schedule the use. Proposals that are active for more than one year must be extended annually by the host, and resubmitted for review after three years. Figure 5-1 depicts the EMSL proposal lifecycle. 


\section{EMSL Resource System}

EMSL's need to manage its assets and systematically track the use of its resources resulted in development of the EMSL Resource System. This system provides the data needed to assure maximum efficiency, effectiveness, and impact of resources.

The EMSL Resource System (Figure 5-2) is an online software tool that is used by EMSL staff to schedule, track, and document the use of major EMSL resources. 'This system is used to generate timely reports providing quarterly and annual resource usage to DOE and other federal agencies. It is also indispensable in assisting EMSL management with making budget decisions for further enhancements, acquisitions, consolidation of current capabilities, and directions for capability growth. Eliminating the need for paper-log entries of use, the EMSL Resource System provides an at-a-glance view of multiple resources and enables flexibility and instantaneous modification of access schedules. Unexpected changes due to instrument failure or user requests for alternate access times can be quickly addressed and reflected by scheduling the user on alternate instruments or swapping and matching users with available time slots.

Figure 5-2.

EMSL Resource Svstem User Interface Visualizing Resource Utilization.

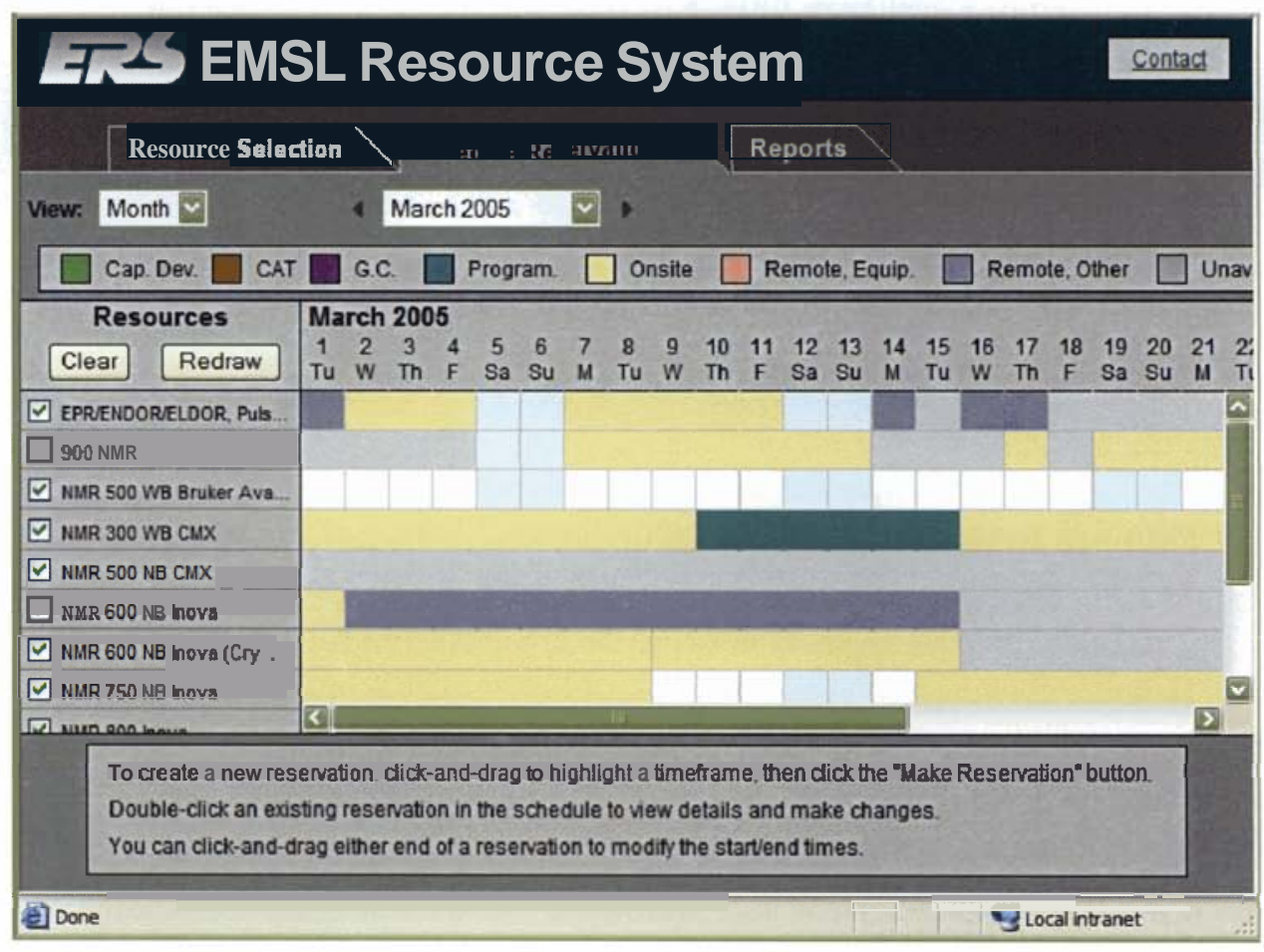




\section{EMSL Proposal Review System}

EMSL will develop a proposal review system designed to automate, manage, and document both the external and internal proposal review process as tailored to the needs of each facility. Existing proposal review software will be reviewed and borrowed from where possible - and then tailored as necessary. The EMSL management team and researchers will be involved in the development of objective proposal ranking criteria, which may require some customization by facility. The resulting software will manage the proposal review lifecycle, potentially including any calls for proposals, communications with internal and external reviewers, external access by reviewers where appropriate, documentation of all review results, and reports summarizing current and past review cycles.

The EMSL Proposal Review System will meet the following objectives:

- Expedite proposal review processes for each facility in a manner that minimizes the burden to the facilities and their reviewers.

- Assist EMSL in ensuring that the science performed at the user facility supports its thrust areas and results in the most impactful science possible.

- Provide statistical summaries that could prove valuable as EMSL makes difficult decisions about which instruments to maintain and upgrade and which to retire.

An electronic review process is a natural extension of the existing EMSL User System and EMSL Resource System, since information already available in those systems can be rolled into the scoring criteria. To manually compile this information on proposals would be time-consuming and would likely result in less accurate scoring. 
6

\section{User Statistics and Demographics}

\section{1}

\section{User Demographics}

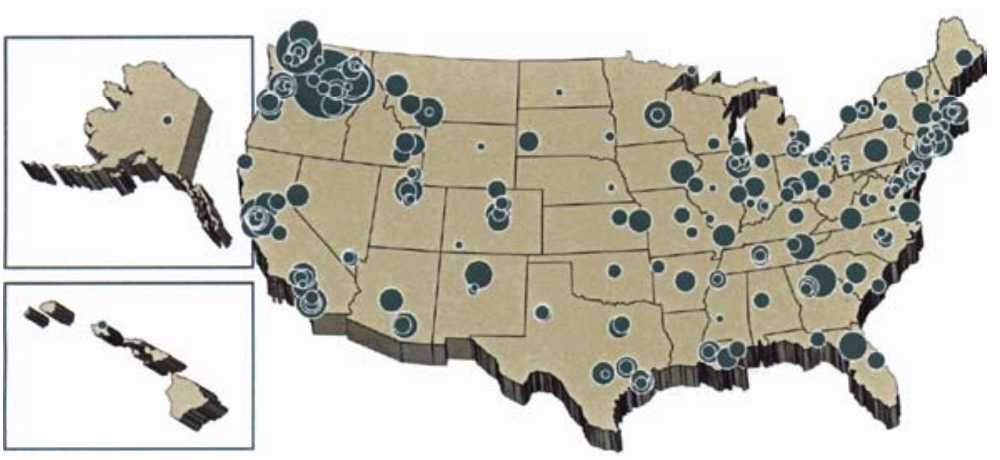

Figure 6-1. Home states of national EMSL users from Fiscal Years 2002 to 2004. Bubble size denotes user numbers from those areas.

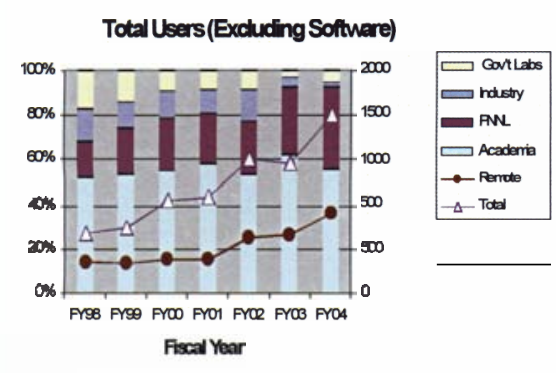

Figure 6-3. User number by affiliation.
Since the doors of EMSL first opened in 1997, its staff have excelled at increasing the total number of users at the facility. To date approximately 7500 scientists (from all 50 states - see Figure 6-1 for a breakdown of users by state, and another 6 percent from foreign countries - see Figure 6-2 for a breakdown of countries representing EMSL users) have used the facility in support of their scientific efforts. On average, use of EMSL capabilities and software has increased about 25 percent annually. During the past seven years, approximately industry decreased from approximately 10 percent to 5 percent. Likewise, there has been a slight decrease in the number of users from other federal laboratories. Lastly, the number of remote users has increased slowly during the past seven years due to an increase in capacity in EMSL's High-Field Magnetic Resonance Facility and Molecular Science Computing Facility, both which enable remote resource use. Figure 6-3 provides a graphical depiction of the number of users by affiliation. In addition, Appendix F provides a listing of users since EMSL opened and their associated projects.

Breaking down EMSL use by facility (Figure 6-4), each facility has steadily grown its user base. For example, the large increase in users of the Molecular Science Computing Facility between Fiscal Years 2003 and 2004 is due to an increase in computational capacity resulting from bringing online EMSL's secondgeneration supercomputer. 'Ihe High-Field Magnetic Resonance Facility has reached saturation and therefore has begun to plateau in terms of total numbers of users, while other facilities continue to grow.

In addition to providing access to the capabilities housed within EMSL, the Molecular Science Computing Facility provides access to and support for the EMSL-developed Molecular Sciences Software Suite, which includes NWChem, Ecce, and GA Tools. These software programs are available for users to download and use at their home institutions, with one license available per institution. Figure 6-5 represents the total cumulative number of approved software agreements. The Molecular Science Computing Facility continues to provide some support of offsite software by maintaining a help queue and sending new versions as they become available. Again, academic institutions far surpass industry and other government laboratories as the main users of EMSL's software capabilities. 
In Fiscal Year 2001, EMSL began to track users based on their scientific discipline (how users identify themselves) in order to gain insight to the types of scientists that use EMSL capabilities. From the data (shown in Figure 6-6), the percentage of chemists using EMSL has remained fairly consistent at 35 percent and environmental scientists remaining constant at about 20 percent of the total users. The percentage of users who are identified as researchers in the life sciences has also stayed constant at 15 percent. The largest growth area of use at EMSL has been in the materials sciences; the percentage of those users has roughly doubled during the past four years.

Figure 6-2.

Home countries of EMSL users from Fiscal Years 2002to 2004. Stars denote user countries.

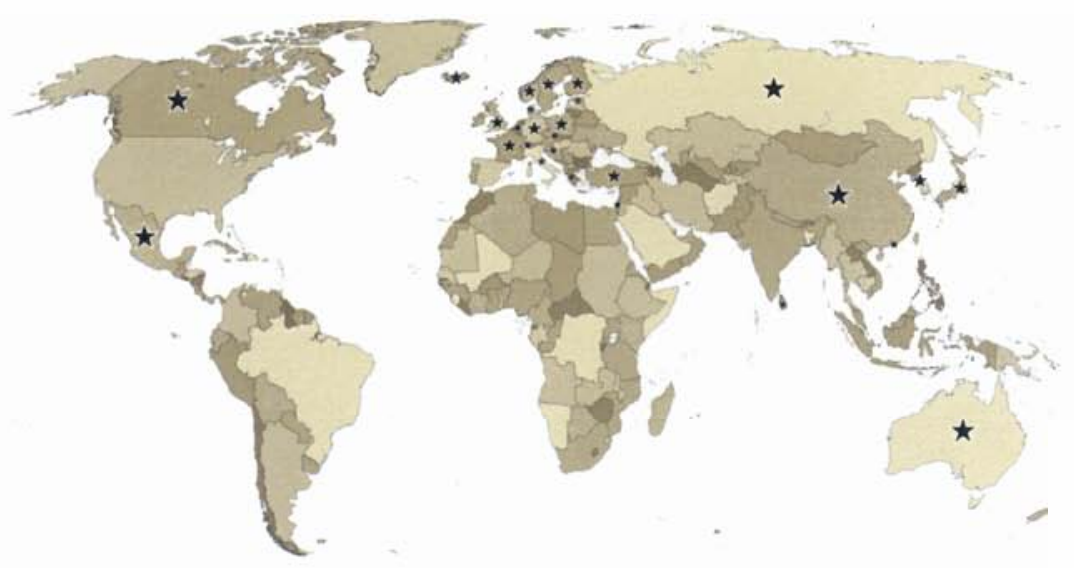

6.2

\section{User Publications}

While user numbers are an important measure of the success of EMSL, it only reflects the quantity of use. EMSL has recently begun to focus efforts on attracting the best possible scientists as users. To that end, in Fiscal Year 2005 EMSL began tracking the number of distinguished users, defined as any of the following:

- A Nobel Laureate (one to date)

- A member of the National Academy of Sciences (one to date)

- An Endowed Chair (seven to date)

- A scientist who is in the top 1 percent of most cited authors as reported by the Essential Science Indicator over a 10-year period (approximately 90 to date).

Measuring the productivity and impact of the science that is accomplished within EMSL is also important in understanding the quality of the science performed, the productivity of the scientists who use EMSL, and the overall value of the facility to enabling cutting-edge scientific discovery. To that end, the publications that result from EMSL user projects are tracked, in addition

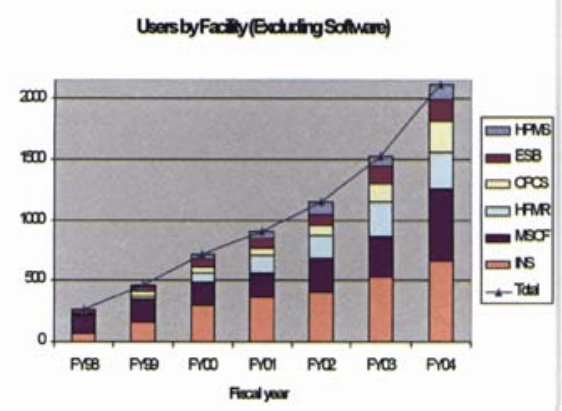

Figure 6-4. EMSL users by facility, excluding software use .

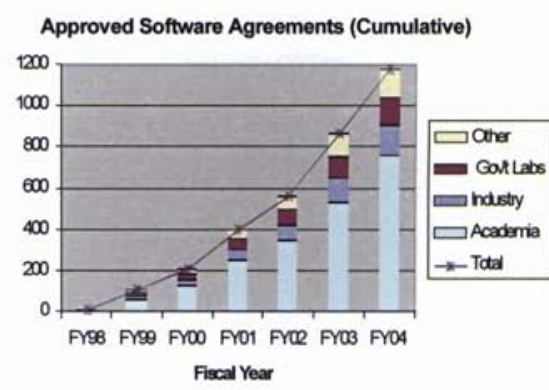

Figure 6-5. Software agreements.
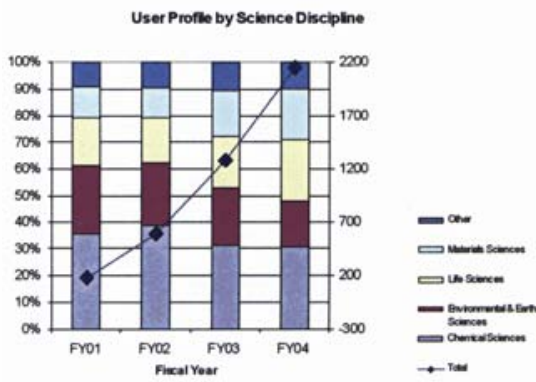

Figure 6-6. User profile by science discipline. 
to the number of journal publications that are published in top-tier journals (any journal listed in the ISI top ten list in a given year) in their respected field. Figure 6-7 shows a graphical depiction of peer-reviewed publications and top10 journals in which research conducted at EMSL was published. In addition, Appendix $\mathrm{G}$ lists the journal publications generated by EMSL users by year, since the facility opened.

While EMSL tries to capture all publications generated as a result of EMSL research, the facility relies heavily on user notification when a paper is published. To ensure capture of all publications, EMSL follows up with all users for up to three years following the end of their project. Additionally, for all projects that users wish to extend, EMSL requests any publications that resulted from the researcher's earlier work. 'These methods have assisted EMSL with gathering the most comprehensive publications list as possible.

\section{Figure 6-7.}

Peer-reviewed publications and top-10 journals in which EMSL research is published, since facility opening.

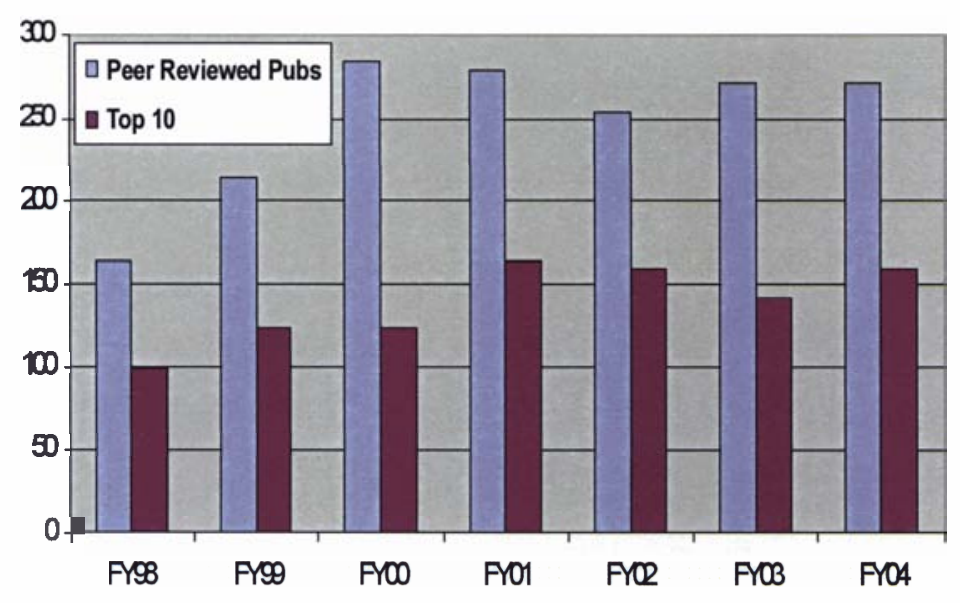

\section{3}

\section{User Patents}

One of EMSL's signature characteristics is the development of new capabilities in support of user science. 'The substantial effort EMSL staff have undertaken to develop new capabilities has resulted in significant intellectual property. Since facility opening, EMSL staff and users have received 95 U.S. patents for their innovative technological advancements (Figure 6-8). 'These patents have enabled EMSL to increase the sensitivity of its FTICR mass spectrometers, develop new chemical detection modalities, and enhance imaging capabilities. A detailed list of patents awarded to EMSL staff and users is contained in Appendix $\mathrm{H}$. 
Figure 6-8.

Graphical depiction of patents awarded to EMSL users and researchers since the facility opened.

Issued Patents

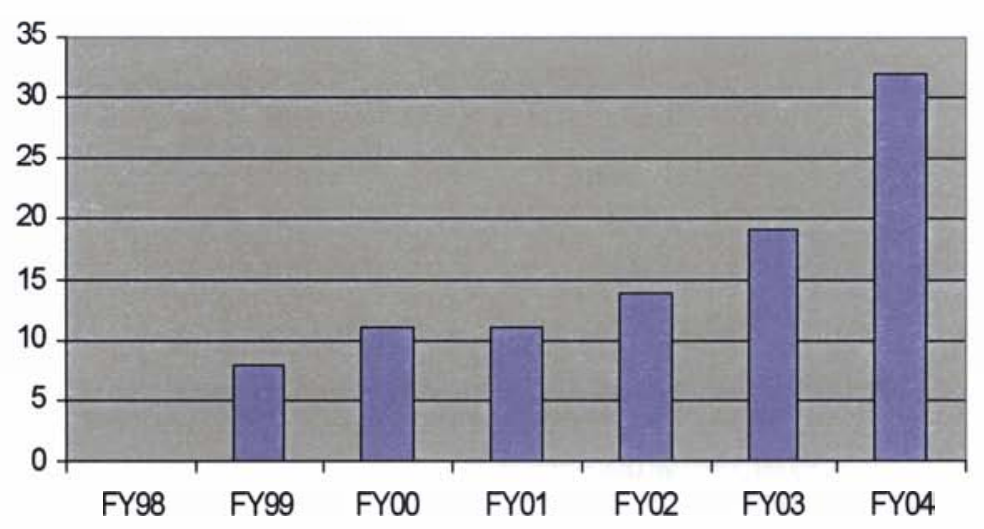




\section{7}

\section{EMSL User Facility Operations Budget}

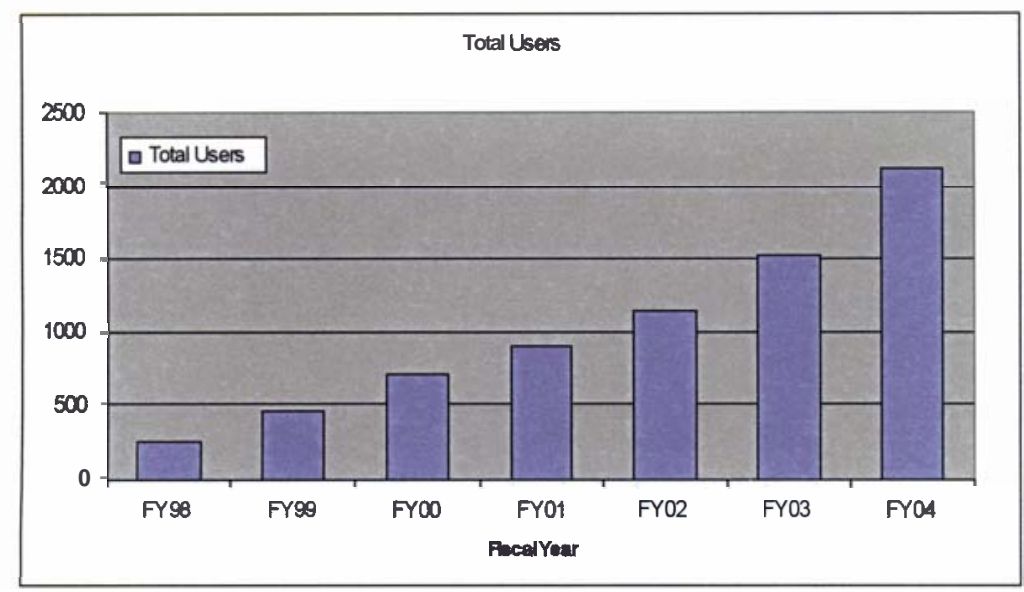

Figure 7-1. Growth of EMSL User Program per fiscal year.
Now entering its eighth year of operation, EMSL has witnessed phenomenal growth of its user program-a sustained growth rate of approximately 25 percent per year (Figure 7-1). In Fiscal Year 2005 alone, more than 2100 scientists from all 50 states and around the world used EMSL's extraordinary capabilities. During its full seven years of operation, EMSL's operations budget has increased from $\$ 29 \mathrm{M}$ to approximately $\$ 40 \mathrm{M}$. 'This increase is predominately due to the additional dedicated funds in support of EMSL's supercomputer. Excluding these fixed costs associated with the supercomputer, the budget has remained relatively constant. As a result of inflation and increased space and labor costs, the "buying power" of the remaining operations budget is less than 84 percent of what it was in Fiscal Year 1998 (Figure 7-2).

Figure 7-2.

EMSL budget by funding type.

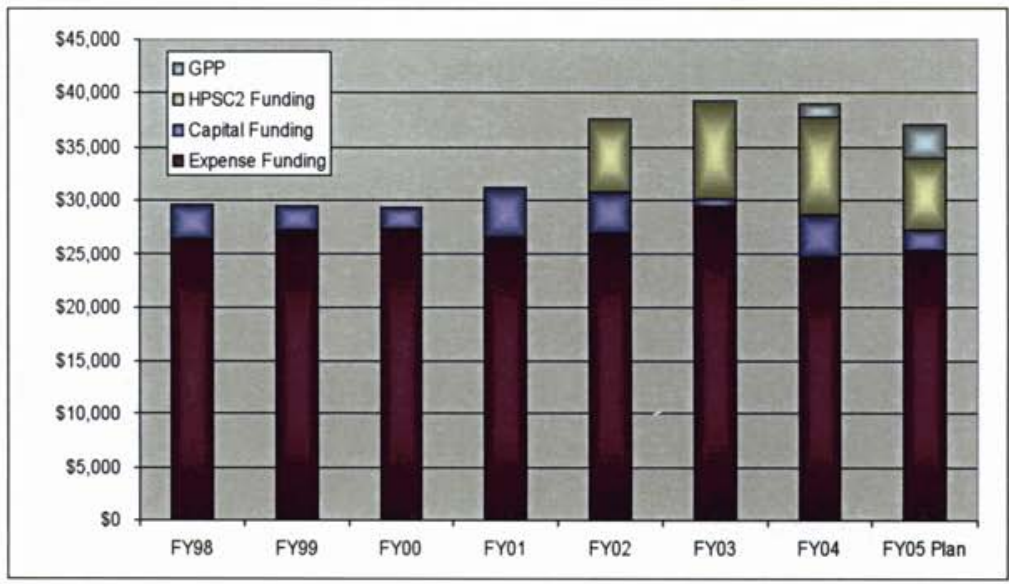

In this climate of continually reduced buying power, serving the user community and increasing the scope of EMSL's capabilities have presented difficult challenges to EMSL management. In order to address these challenges, EMSL management has during the past few years taken a hard look at expenses in an attempt to reduce cost by increasing efficiency. Resulting actions taken involved streamlining EMSL's senior management team by reducing the number of staff supporting the EMSL Director's Office, eliminating some service contracts, and reducing procurements. 'This has enabled EMSL to shift all available resources to labor for its scientific 
consulting staff in support of EMSL's user program (Figure 7-3). Additionally, in Fiscal Year 2005, EMSL management asked PNNL for a change in space charging that resulted in a savings of approximately $\$ 1 \mathrm{M}$ to the EMSL operations budget. This savings will used to make investments in capital equipment and capability development.

In addition to the streamlining discussed above, in Fiscal Year 2003, EMSL management decided to reduce the amount of total funding allocated to the individual facilities in order to generate a pool of funding that could be used for targeted capability developmentactivities and strategic investments, such as the Scientific Grand Challenges and CATs (Figure 7-4). Investment decisions are now made according to criteria such as:

- alignment with EMSL's mission

- benefit to users

- alignment with scientific strengths

- uniqueness of the proposed effort

- cross-cutting capability.

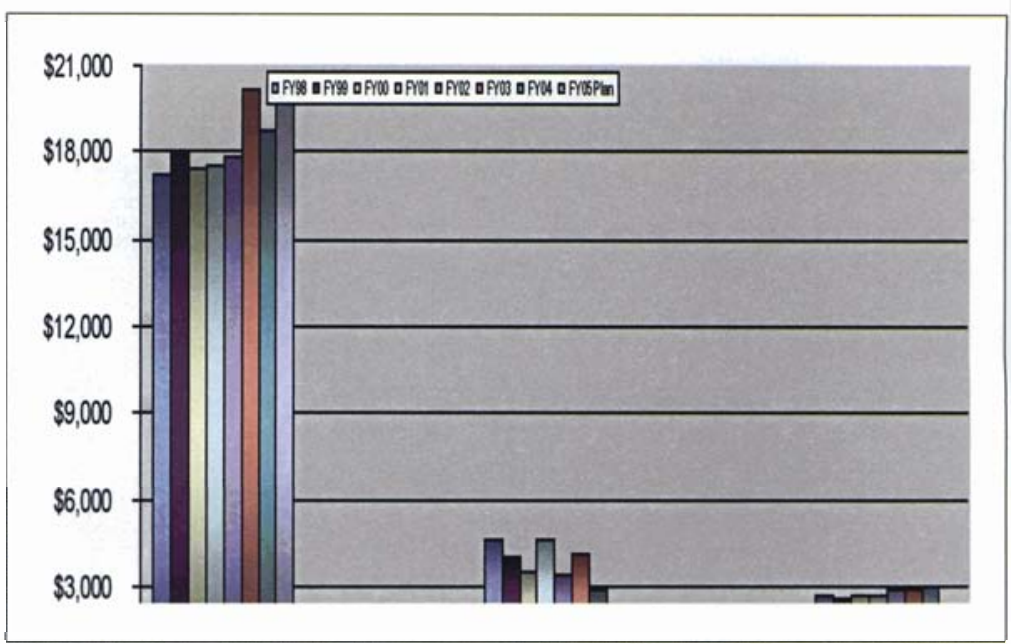

Figure 7-3. Funding shift from items such as travel, procurements, subcontracts, and space, to labor.

'The user facility has also been working hard to leverage other investments with EMSL capital in order in refresh capabilities. This has substantially helped in maintaining instrumentation at state of the art. As shown in Figure 7-5, EMSL has had significant investment from DOE's Office of Basic Energy Sciences. In addition, investments from the National Institutes of Health and DOE's National Nuclear Security Administration have resulted in additional capabilities in high-resolution Fourier transform Infrared spectroscopy, NMR, and mass spectrometry.

Figure 7-4.

EMSL strategic investment.

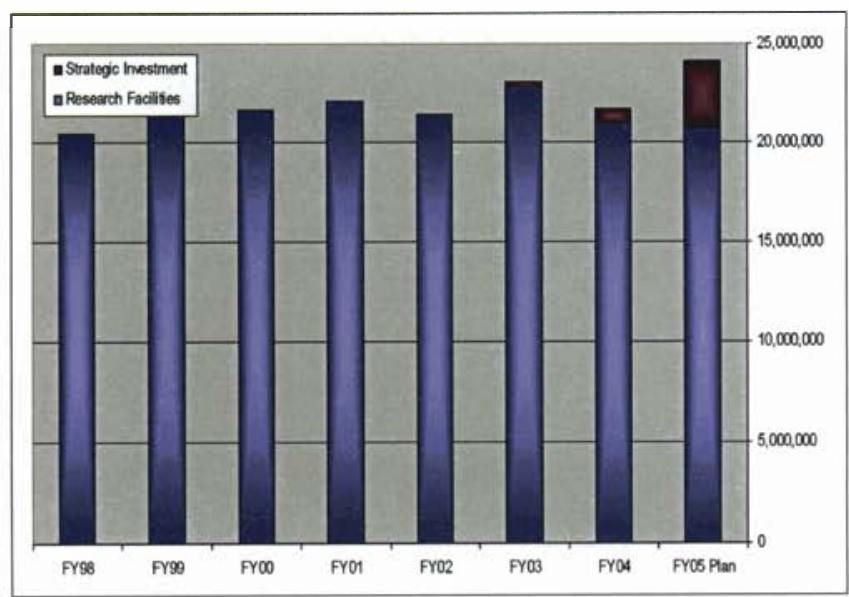




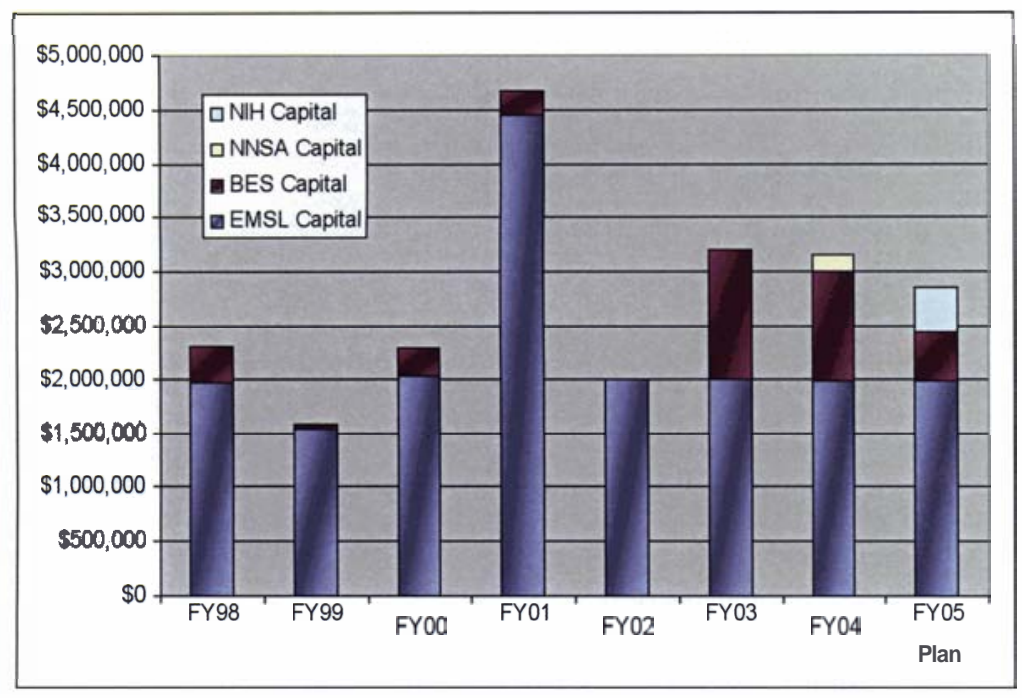

Along with investments from DOE and other agencies, PNNL has provided investment dollars to EMSL (Figure 7-6), ranging from LaboratoryDirected Research and Development (LDRD - used to build new capabilities), Program Management and Development (PDM - used to develop new programmatic opportunities), PNNL Overhead (PNNL OH -used to support and enhance laboratory infrastructure), General Plant Project (GPP - used for facility infrastructure improvements), General Research Equipment (GRE - used to procure equipment that enhances laboratory research capabilities), and Planning and Administration (P\&TL - used for developing new plans for future programmatic work). As shown in Figure 7-6, PNNL substantially decreased its investments to EMSL as a result of a reorganization in which many EMSL staff were moved organizationally to another research directorate.

Figure 7-6.

PNNL investment to EMSL by type.

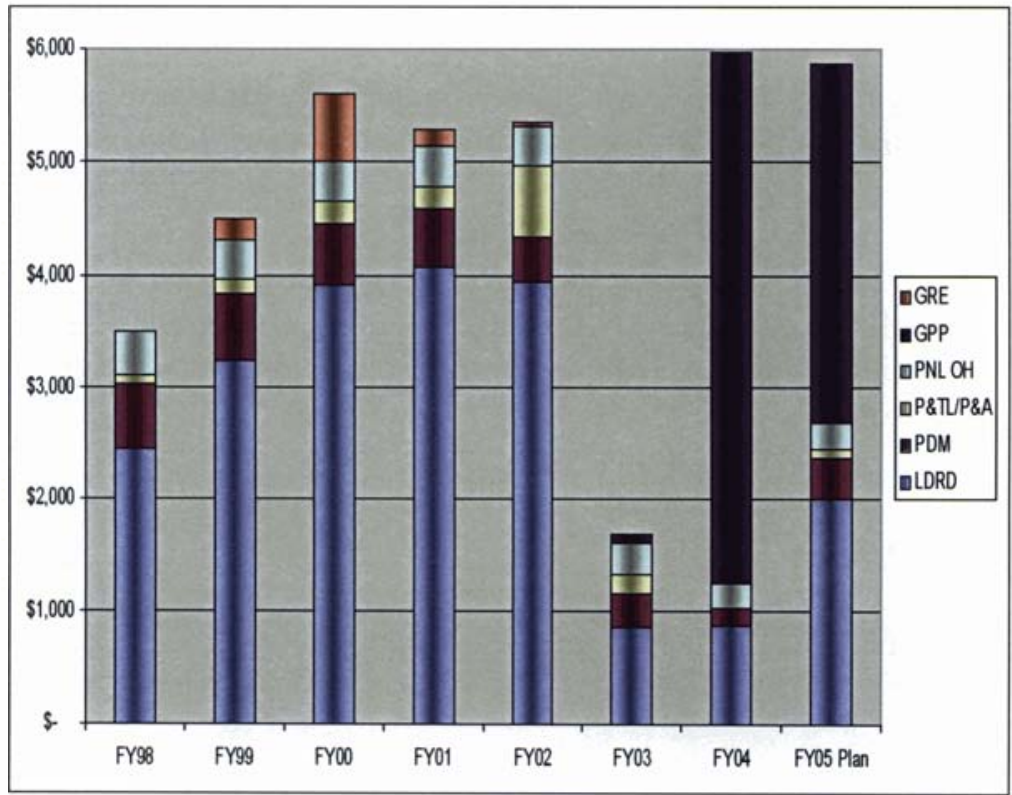


8

\section{Appendices}

This EMSL Outlook Review contains nine appendices that support the content of this document. 'These appendices are included on the CD that accompanies this document. 'They consist of the following:

\section{Appendix A}

Detailed Roles, Responsibilities, Accountabilities, and Authorities of EMSL Management

\section{Appendix B}

Detailed Descriptions of EMSL's Six Research Facilities

\section{Appendix C}

List of EMSL Instruments and Equipment

\section{Appendix D}

CVs and Bios of Key EMSL Staff

\section{Appendix E}

EMSL User Testimonials by Facility

\section{Appendix F}

List of EMSL Users and Projects by Year

\section{Appendix G}

List of Publications Generated by EMSL Research

\section{Appendix $\mathrm{H}$}

List of Patents Received as a Result of EMSL Research 